\title{
Polarization Dependence of Two-Photon Absorption in Solids
}

by

Todd Richard Bader

\author{
Submitted in Partial Fulfillment \\ of the \\ Requirements for the Degree \\ DOCTOR OF PHILOSOPHY
}

Supervised by Professor Albert Gold

Institute of Optics

The University of Rochester

Rochester, New York 
Vita

The author was born in Albany, New York on February 12, 1943. He did his undergraduate work in physics at the State University of New York at Albany, where he received his bachelor's degree in 1965.

In 1965 the author began his graduate study at the Institute of Optics at the University of Rochester. During the academic years 1965-1967 and 1968-1969 he was a graduate teaching assistant. He served as a research assistant in 1967-1968. 
Acknowledgments

The author would like to thank Professor Albert Gold for his suggestion of the problem and for his continuing interest and participation in this work. The interest of Professor Robert $S$. Knox and his critical reading of parts of the manuscript are greatly appreciated.

The author would like to thank Professor M. Inoue and Professor Y. Toyozawa for their most helpful correspondence.

Many stimulating and informative discussions, especially on the experimental aspects of two-photon absorption, with Mr. Terrence H. Reilly have been of invaluabie aid throughout the course of this work.

The author would also like to thank his wife, Sharon, for her rather blind but stimulating interest, and for typing the thesis.

This research was supported in part by a grant fro the National Science Foundation. 
Abstract

The dependence of two-photon absorption in solids on the polarization of the exciting photons has recently been studied, both experimently and theoretically, as a means of identifying electron state symmetries. Theoretically; polarization dependence has been calculated for allowed two-photon transitions at the $\Gamma$-point from a totally symmetric ground state to a final state of arbitrary symmetry for each of the thirty-two point groups and at other symmetry points for several crystal structures. More recently, polarization dependence has been studied for forbidden transitions in cubic structures in a two spherical band model with the inclusion of exciton effects in the intermediate and final states. In this model the polarization dependence was found to be independent of one-electron state symmetries or the orientation of photon polarizations with respect to the crystal axes.

This work extends the previous investigations in several respects. Mathematical expressions and tables are obtained for allowed $\Gamma$-point transitions between states of arbitrary symmetry for the thirty-two point groups and for transitions at other symmetry points in hexagonal close-packed and wurtzite structures. For 
forbidden transitions a simpler band model is used neglecting exciton effects. This predicts a polarization dependence similar to, but more easily evaluated than, the earlier result; the ratio of maximum to minimum absorption predicted by the earlier calculation is a complicated function of the band gap and exciton binding energies, which usually are not precisely known, while in the simpler model this ratio is a simple function of the photon energies. The effects of $k \cdot p$ splitting of $p$-like valence bands is considered in this model, and it is found that only the ratio of maximum to minimum is altered. In addition the polarization dependence is calculated for forbidden $\mathrm{X}$-point transitions in the two band model, but in which the ellipsoidal character of energy surfaces near this point are taken into account. The two-photon absorption is found to depend both on the symmetries of the initial and final states and on the orientation of the polarization vectors with respect to the crysotal axes. 
Table of Contents

Chapter

Page

I. Introduction . I

II. Two-Photon Absorption 7

A. Perturbation Theory . 7

B. Application to Solids 16

III. Allowed Transitions 35

A. Formalism 36

B. $\Gamma$-Point Transitions 44

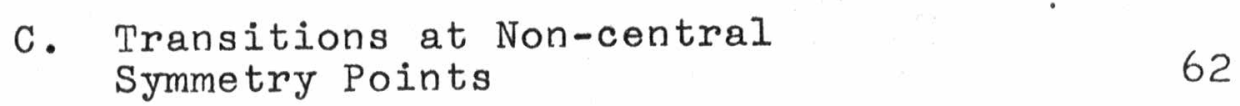

IV. Forbidden Transitions 82

A. Mahan's Model 83

B. Band Model 92

C. p-like Valence Bands 97

D. X-Point Transitions 114

V. Application to Experiment 120

A. $\mathrm{CuCl} \quad 121$

B. ZnS 127

C. $\mathrm{RbI} \quad 131$

D. CsI 134

- E. KI 137

F. TICI 144 
Chapter

VI. Conclusion

146

Appendices

A. $k \cdot p$ Perturbation Theory 149

B. Symmetry of $\wedge(\omega) \quad 151$

C. Derivation of Clebsch-Gordan 153

D. Table of Correspondence of

Point Group Notations

156

E. Angular Functions for Symmetry Points in Face-Centered Cubic, Diamond, Zinc Blende and Simple Cubic Structures

References 
viii

\section{Iist of Tables}

Table No.

Page

3-1 Angular Dependence Functions

for $\Gamma$-Point Transitions

46

3-2 Complete Character Table for the Double Group of $A$ in Hexagonal Close-Packed Structures

3-3 Angular Dependence Functions for Hexagonal Close-Packed Structures

3-4 Angular Dependence Functions for Wurtzite structures

D-1 Correspondence of Point Group Notations

E-1 Angular Dependence Functions for Face-Centered Cubic, Diamond, Zinc Blende and Simple Cubic 


\section{List of Figures}

Figure No.

Page

2-1 Paths of forbidden two-photon

absorption

3-1 Definition of axes for point groups $\mathrm{D}_{2}, \mathrm{D}_{2 \mathrm{~h}}, \mathrm{C}_{2 \mathrm{v}}, \mathrm{D}_{4}, \mathrm{C}_{4 \mathrm{v}}$ and $\mathrm{D}_{4 \mathrm{~h}}$

58

3-2 Definition of axes for point groups $\mathrm{D}_{2 \mathrm{~d}}, \mathrm{D}_{3}, \mathrm{D}_{3 \mathrm{~d}}, \mathrm{C}_{3 \mathrm{v}}$ and $\mathrm{D}_{6}$

3-3 Definition of axes for point groups $\mathrm{C}_{6 \mathrm{v}}, \mathrm{D}_{6 \mathrm{~h}}$ and $\mathrm{D}_{3 \mathrm{~h}}$

60

3-4 Definition of axes for point groups $\mathrm{T}, \mathrm{T}_{\mathrm{d}}, \mathrm{T}_{\mathrm{h}}, \mathrm{O}$ and $\mathrm{O}_{\mathrm{h}}$

3-5 Brillouin zone of hexagonal closepacked and wurtzite structures

4-1 Two-photon absorption for spherical p-like bands

4-2 Ratio of parallel to perpendicular polarization absorption for $\mu_{1}>\mu_{2}$

4-3 Ratio of parallel to perpendicular polarization absorption for $\mu_{2}>\mu_{1}$

4-4 Constant energy surfaces near $\mathrm{X}$-point extremum

$\begin{array}{lll}5-1 & \text { Band structure of CuCl. } & 124\end{array}$

5-2 Two-photon absorption spectrum of CuCl 125

$\begin{array}{ll}\text { 5-3 Polarization dependence of two-photon } & \\ \text { absorption in CuCI } & 126\end{array}$

5-4 Band structure of $\mathrm{ZnS}$ 
Figure No.

Page

5-5 Polarization dependence of two-photon absorption in $\mathrm{ZnS}$

5-6 Two-photon absorption in RbI.

133

5-7 Two-photon absorption in CsI

135

5-8 Band structure of $\mathrm{CsI}$

136

5-9 Band structure of $\mathrm{KI}$

141

5-10 Two-photon absorption spectrum of KI

142

5-11 Polarization dependence of two-photon absorption in $\mathrm{KI}$

143

E-I Brillouin zones of simple cubic, diamond, zinc blende, face-centered cubic and body-centered cubic structures 
Chapter I

Introduction

Spectroscopy, the study of the interaction of light with matter, provides many useful methods of investigating the physical properties of atoms, molecules, and solids. In the study of insulators and semiconductors, with which this dissertation is concerned, substantial information on photon absorption (and hence, for example, exciton, impurity state and band energies) has been gained with the use of now commonplace spectroscopic techniques. 1 Response functions, such as the polarizability, in this ordinary spectroscopy are proportional to the electric field. Only with very large fields are nonlinear effects observable. Such light sources were not available until the early 1960's. With the advent of the laser nonlinear spectroscopy quickly became an area.of wide interest. 2

The nonlinear process of two-photon absorption was first discussed by Goeppert-Mayer ${ }^{3}$ long before the laser was developed. Two-photon absorption techniques were developed by Hopfield and Worlock ${ }^{4}$ for the study of solids. Such techniques are useful in studying the electronic states of solids in several ways. Suppose a crystal having inversion symmetry has a minimum vertical band gap $\mathrm{E}_{\mathrm{g}}$ at 
$\vec{k}=0$. The shape of the absorption edge in this crystal is described approximately by $\left(\hbar \omega-E_{g}\right)^{3 / 2}$ for one-photon absorption and by $\left(\hbar \omega_{1}+\hbar \omega_{2}-E_{g}\right)^{1 / 2}$ for two-photon absorption, where $\hbar \omega$ is the energy of a photon, if the valence and conduction bands have the same parity. The two-photon process in this case allows a better examination of states having energy near $\mathrm{E}_{\mathrm{g}}$, and permits excitons constructed from states in these bands to be observed. In the more general case the two-photon absorption data provides a supplement to the one-photon data. The one-photon absorption coefficient is often so large that the experimentalist must employ reflectivity techniques ${ }^{5}$ or use a very thin crystalline sample, 6 the results of which exhibit effects (though they are usually assumed to be small) due to the surface. 7-9 Large samples can be used in the two-photon process; and the absorption takes place in the heart of the crystal, where the Born-von Karman boundary conditions used in band theory are better approximated. Yet another premium can be placed on the utilization of two-photon spectroscopy in the study of solids. The dependence of the absorption coefficient on the directions. of polarization of the two photons can provide useful information on the symmetries of the conduction and valence bands. Dresselhaus ${ }^{10}$ emphasized the fact that the anisotropic one-photon absorption in anisotropic crystals could be explained by group theoretical considerations. Consid- 
erably more extensive information, however, can be obtained from the two-photon spectra of solids, including those which are one-photon isotropic, by examining the absorption coefficient as a function of the directions of polarization of two incident beams. The form of this angular dependence function will depend on the irreducible representations of the crystallographic point group to which the initial and final states belong. Inoue and Toyozawa ${ }^{11}$ first drew attention to this fact and calculated the functions for all final crystal state symmetries for each of the thirty-two point groups. They also calculated the angular functions for transitions at points and on lines of symmetry in the Brillouin zone other than $\vec{k}=0$ for $\mathrm{NaCl}, \mathrm{CsCl}$, diamond, and zinc blende structures. Their results give directly the angular function for a transition between one-electron states of arbitrary symmetry only in the case of allowed two-photon transitions in which either the valence band: or the conduction band belongs to the identity representation of the group of the wave vector, and, in the case of excitons, when the envelope function belongs to the identity representation.

It often happens that one-electron transitions are forbidden (by parity, for example) at an energy band extremum. The "forbidden" transitions observed in the optical spectra take place on an energy surface in $\vec{k}$-space enclosing the extremum. The two-photon absorption 
coefficient for these transitions has been calculated by Mahan 19, 20 in a two band model in which the valence and conduction bands are assumed to be spherical near the extremum. Exciton effects were explicitly included in his calculation. Mahan found that the absorption coefficient does not depend on the directions of polarization with respect to the crystal axes, but only on the angle between the two polarization vectors. The absorption coefficient has the form $a \cos ^{2} \theta+b \sin ^{2} \theta$. The ratio $a / b$ is difficult to evaluate and it depends on quantities which are not always precisely known. Angular dependence measurements ${ }^{12-18}$ have been made on crystals in which the observed transitions are of the allowed kind and on crystals in which the transitions are forbidden. These measurements have all been discussed by their investigators in the light of the theoretical works of Inoue and Toyozawa ${ }^{11}$ and of Mahan. 20

In this dissertation the angular functions will be produced for transitions in which the conduction band and valence band at the center of the Brillouin zone are of arbitrary symmetry for each of the thirty-two point groups. A way of finding the angular dependence of transitions between states of arbitrary symmetry at other high symmetry points in the Brillouin zone will be given which utilizes the $\Gamma$-point tables and the tables of Inoue and Toyozawa for the non-central symmetry points. Also, the angular 
functions for symmetry lines and points of the hexagonal close-packed and wurtzite structures will be calculated. The results will apply to band-to-band transitions and exciton transitions when the envelope function belongs to the identity representation. For forbidden transitions a simpler model will be considered in which exciton effects are ignored, but in which the restriction to two spherical bands is retained. It is shown that this model leads to a polarization dependence similar to that obtained by Mahan but for which the ratio $a / b$ is a simple function of the photon energies only. The effect on the polarization dependence of $\overrightarrow{\mathrm{k}} \bullet \overrightarrow{\mathrm{p}}$ splitting near the $\Gamma$-point is considered in the particular case of p-like valence bands and a totally symmetric conduction band, and it is shown that this band splitting does not alter the form of the polarization dependence, but changes the expression for the ratio $a / b$. Finally, the polarization dependence will be determined for forbidden transitions at the $\mathrm{X}$-point in simple cubic and face-centered cubic structures, about which the constant energy surfaces are ellipsoidal. For these transitions it is found that the absorption coefficient depends on the symmetries of the initial and final states. and on the orientations of the polarization vectors with respect to the crystal axes.

In Chapter II a review of the perturbation treatment of two-photon absorption will be given with its application 
to the process in solids. Chapter III contains the. formalism used to obtain the angular functions for allowed transitions and includes the results for the $\Gamma$-point and non-central symmetry points. Chapter IV is devoted to forbidden transitions, containing a review of Mahan's 20 results and the calculations neglecting exciton effects. A discusision of some of the experimental work $12-17$ in terms of theoretical results is presented in Chapter $V$. 


\section{Chapter II}

\section{Two-Photon Absorption}

\section{- A. Perturbation Theory}

The process of two-photon absorption by an atomic system can be treated with the use of second order timedependent perturbation theory 21,22 The specific process of interest is the following: we imagine that we have an atomic system, such as a crystalline solid, in its lowest energy state. Two Iinearly polarized light beams are incident on the system, each possessing a narrow spread in frequency. The atomic system is such that it has no excited states near energy $\hbar \omega_{1}$ or $\hbar \omega_{2}$ the photon energies of light beams one and two, respectively. But it does have an excited state near $\hbar \omega_{1}+\hbar \omega_{\lambda}$. In solids it is generally impossible to avoid a resonance at energy $2 \hbar \omega_{1}$ or $2 \hbar \omega_{2}$. We must therefore stipulate that in a two beam experiment, one beam must have insufficient power to cause detectable absorption of two photons from the same beam, say beam one. This will be accomplished with the use of a "conventional" light source. Beam two, a laser, must be sufficiently potent to allow the detection of absorption of two photons, one each from beams one and two, and hence it is imperative to avoid a 
resonance at $2 \hbar \omega_{2}$. This is generally accomplished by choosing the laser (or the crystal) such that $\hbar \omega_{2}$ is less than half the energy of the lowest excited state of the crystal.

The Hamiltonian of the entire system, in the coulomb gauge, is

$$
\begin{aligned}
H & =\sum_{j=1}^{N} \frac{1}{2 m}\left[\vec{p}_{j}-\frac{e}{c} \vec{A}\left(\vec{r}_{j}\right)\right]^{2}+V\left(\vec{r}_{1}, \vec{r}_{2} \cdots \vec{r}_{N}\right)+H_{F} \\
& =H_{0}-\frac{e}{m c} \sum_{j} \vec{A}\left(\vec{r}_{j}\right) \cdot \vec{p}_{j}+\frac{e^{2}}{2 m c^{2}} \sum_{j} A^{2}\left(\vec{r}_{j}\right)
\end{aligned}
$$

where $H_{0}=H_{A}+H_{F}$ and $j$ runs over ali electrons. $H_{A} \equiv \frac{1}{2 m} P^{2}+V$ is the energy of the unperturbed atomic system, with $\vec{P} \equiv \sum_{j} \vec{p}_{j}^{0} \cdot H_{F}$ is the free photon field Hamiltonian. It is not necessary to use the quantized field formalism for absorption processes, 3,24 but we shall employ it here simply for convenience. 25 In this description the vector potential is given by

$\vec{A}\left(\vec{r}_{j}\right)=c \sum_{k, \sigma} \sqrt{\frac{2 \pi \hbar}{\omega_{k} V k_{k \sigma}}} \hat{\varepsilon}_{k \sigma}\left[a_{k \sigma} e^{i \vec{k} \cdot \vec{r}_{j}}+a_{k \sigma}^{\dagger} e^{-i \vec{k} \cdot \vec{r}_{j}}\right]$,

where $\vec{k}=\frac{2 \pi}{v^{1 / 3}}\left(l_{1} \hat{x}+l_{2} \hat{y}+l_{3} \hat{z}\right) \quad\left(l_{i}=\right.$ integer $)$ and $\sigma$ runs over two polarizations. $\quad H_{F} \equiv \sum_{k \sigma} \hbar \omega_{k}\left[a_{k \sigma}^{+} a_{k \sigma}+\frac{1}{2}\right]$ is a sum of harmonic oscillator Hamiltonians, each of which has eigenstates such that

$$
a_{k \sigma}\left|n_{k \sigma}\right\rangle=\sqrt{n_{k \sigma}+1}\left|n_{k \sigma}+1\right\rangle
$$


and

$$
a_{k \sigma}^{+}\left|n_{k \sigma}\right\rangle=\sqrt{n_{k \sigma}}\left|n_{k \sigma}-1\right\rangle \text {. }
$$

The energy of state $\left|n_{k \sigma}\right\rangle$ is $\hbar \omega_{k}\left(n_{k \sigma}+\frac{1}{2}\right) . a_{k \sigma}$ and $a_{k \sigma}^{\dagger}$ are seen to be annihilation and creation operators, respectively, and hence will correspond respectively to absorption and emission of photons. The total field eigenstate can then be written $\left|n_{1}, n_{2}, \ldots n_{i} \ldots\right\rangle$, where $n_{i}(i \equiv k, \sigma)$ represents the number of photons of type $i$ which are present in the field. The eigenstates of $H_{0}$ can now be written in the form

$$
|\alpha\rangle=|a\rangle\left|n_{1}, n_{2}, \cdots n_{i}, \cdots\right\rangle
$$

where $|a\rangle$ are eigenstates of $H_{A}$ with energy $E_{a} \cdot|\alpha\rangle$ has energy $E_{\alpha}=E_{a}+\sum_{i} \hbar \omega_{i}\left(n_{i}+\frac{1}{2}\right)$.

As described earlier, light beam one is composed of photons having polarization $\hat{\varepsilon}_{1}$, having direction of propogation very nearly $\hat{k}_{1}$, and having energy within a narrow envelope about $\hbar \omega_{1}$. Beam two is similarly described. Thus the initial state of the system can be written

$$
\begin{aligned}
& |I\rangle \equiv \mid g ; 0,0, \ldots n_{11}, n_{12}, \ldots o \ldots \\
& \cdots n_{21}, n_{22}, \cdots n_{2 j} \cdots 0 \cdots>
\end{aligned}
$$

where $n_{1 i}$ is the number of photons with polarization having $\hat{k}_{i}$ and $\omega_{i}$ very nearly $\hat{k}_{1}$ and $\omega_{1}$, and similarly for $n_{2 j}$. The final state is $|F\rangle \equiv\left|f ; 0,0, \cdots n_{11}, n_{12} \cdots \dot{n}_{1 i}-1, \cdots 0 \cdots n_{21}, n_{22} \cdots n_{2 j}-1, \cdots 0 \cdots\right\rangle$ 
Following the standard time-dependent perturbation theory, the state of the entire system at a time $t$ is

$$
|\psi(t)\rangle=\sum_{\alpha} c_{\alpha}(t) e^{-i E_{\alpha} t / \hbar}|\alpha\rangle
$$

$|\psi(t)\rangle$ satisfies

$$
i \hbar \frac{\partial}{\partial t}|\psi(t)\rangle=\left(H_{0}+H^{\prime}\right)|\psi(t)\rangle
$$

where $H^{\prime}$ is the last two terms of Eq.2-l. $C_{\alpha}(t)$ satisfies

$$
i \hbar \frac{d c_{\alpha}}{d t}=\sum_{\beta}\left\langle\alpha\left|H^{\prime}\right| \beta\right\rangle c_{\beta}(t) .
$$

$\left|C_{\alpha}(t)\right|^{2}$ is the probability of finding the system in state. $|\alpha\rangle$ at time $t$, subject to the condition $c_{\alpha}(0)=\delta_{\alpha I}$. Expanding $c_{\alpha}(t)$ in a power series in the perturbation constant $\lambda$, we have $c_{\alpha}(t)=\sum_{n=0}^{\infty} c_{\alpha}^{(n)}(t)$, where $c_{\alpha}^{(n)}(t)$ is of order $\lambda^{n}$. Substituting into Eq.2-3 and equating equal powers of $\lambda$, we obtain

$$
\begin{aligned}
& i \hbar \frac{d c_{\alpha}^{(0)}}{d t}=0 \\
& i \hbar \frac{d c_{\alpha}^{(n)}}{d t}=\sum_{\beta}\left\langle\alpha\left|H^{\prime}\right| \beta\right\rangle e^{i \omega_{\alpha \beta} c_{\beta}^{(n-1)}(t), \quad n>0 .}
\end{aligned}
$$

We find immediately that

$$
c_{\alpha}^{(0)}(t)=c_{\alpha}^{(0)}(0)=\delta_{\alpha I}
$$

and

$$
i \hbar \frac{d c_{\alpha}^{(1)}}{d t}=\left\langle\alpha\left|H^{\prime}\right| I\right\rangle e^{i \omega_{\alpha I} t}
$$


so that

$$
c_{\alpha}^{(1)}(t)=-\frac{1}{\hbar \omega_{\alpha I}}\left\langle\alpha\left|H^{\prime}\right| I\right\rangle\left[e^{i \omega_{\alpha I} t}-1\right]
$$

We will restrict ourselves to systems, or portions of systems, in which $e^{i \vec{k} \cdot \vec{r}_{j}} \approx 1$. Since we are interested in an absorption process, the vector potential becomes in the dipole approximation

$$
\vec{A}\left(\vec{r}_{j}\right)=c \sum_{k \sigma} \sqrt{\frac{2 \pi \hbar}{\omega_{k} V k_{k \sigma}}} \hat{\varepsilon}_{k j \sigma} a_{k \sigma} .
$$

In the case of a solid the dielectric constant $K$ will sometimes depend on the direction of polarization. We will make a requirement here that in an experiment $\hat{\varepsilon}$ is to be chosen so that $K$ will remain constant. For example, in a hexagonal crystal $\hat{\varepsilon}_{1}$ can be chosen to lie in the direction of the c-axis, while $\hat{\varepsilon}_{z}$ may be varied in the plane perpendicular to the c-axis. The subscript $\sigma$ will now be dropped from $K_{k \sigma}$.

In $H^{\prime}$ the patio of the term quadratic in $A$ to the term linear in $A$ is of the order $\frac{e}{c p} A$, which is small. The term quadratic in A cannot in this approximation connect states with different electronic states anyway. Therefore we will drop this term from $H^{\prime}$, and the effective perturbation becomes

$H^{\prime}=-\frac{e}{m c} \vec{A} \cdot \vec{P}=-\frac{e}{m} \sum_{k \sigma} \sqrt{\frac{2 \pi \hbar}{\omega_{k} V K_{k}}} a_{k \sigma} \hat{\mathcal{E}}_{k \sigma} \cdot \vec{P}$. 
From Eq.2-4 it is seen that $\left\langle\alpha\left|H^{\prime}\right| I\right\rangle$ is non-zero only if a single photon is absorbed. Since there is no atomic state such that $E_{a}-E_{i}=\hbar \omega_{1}$ or $\hbar \omega_{2}$, then $\hbar \omega_{\alpha I}=E_{a}-E_{i}-\hbar \omega_{1}$ or $E_{a}-E_{i}-\hbar \omega_{2}$ is appreciably different from zero, and $\left|C_{\alpha}^{(1)}(t)\right|^{2}$ is very small, oscillating rapidly in time. We find $\begin{aligned} i \hbar \frac{d}{d t} c_{F}^{(2)}(t) & =\sum_{M}\left\langle F\left|H^{\prime}\right| M\right\rangle e^{i \omega_{F M} t} c_{M}^{(1)}(t) \\ & =-\frac{1}{\hbar} \sum_{M}\left\langle F\left|H^{\prime}\right| M\right\rangle\left\langle M\left|H^{\prime}\right| I\right\rangle e^{i \omega_{F M} t} \frac{e^{i \omega_{M I} t}-1}{\omega_{M I}}\end{aligned}$

and

$$
=-\frac{1}{\hbar} \sum_{M}\left\langle F\left|H^{\prime}\right| M\right\rangle\left\langle M\left|H^{\prime}\right| I\right\rangle e^{i \omega_{F M} t} \frac{e^{i \omega_{M I} t}-1}{\omega_{M I}}
$$

$$
C_{F}^{(2)}(t)=\frac{1}{\hbar^{2}} \sum_{M} \frac{\left\langle F\left|H^{\prime}\right| M\right\rangle\left\langle M\left|H^{\prime}\right| I\right\rangle}{\omega_{M I}}\left[\frac{e^{i \omega_{F I} t} \mid}{\omega_{F I}}-\frac{e^{i \omega_{F M} t}-1}{\omega_{F M}}\right] .
$$

By the hypothesis that $E_{f g}=\hbar \omega_{1}+\hbar \omega_{2}, \quad \omega_{F I} \approx 0$. We may neglect the second term in the brackets since it is never resonant.

$$
\left|C_{F}^{(2)}(t)\right|^{2}=\frac{1}{\hbar^{2}}\left|\sum_{M} \frac{\left\langle F\left|H^{\prime}\right| M\right\rangle\left\langle M\left|H^{\prime}\right| I\right\rangle}{E_{M}-E_{I}}\right|^{2} \frac{\sin ^{2} \frac{1}{2} \omega_{F I} t}{\left(\frac{1}{2} \omega_{F I}\right)^{2}}
$$

The final state $|F\rangle$ has two photons less than the initial state $|I\rangle$, one from beam one, the other from beam two. The intermediate state $|M\rangle$ must have one photon less than $|I\rangle$, but it can be one of energy near $\hbar \omega_{1}$ or near $\hbar \omega_{2}$. Thus two terms of the sum in Eq.2-4 survive, and Eq.2-5 reduces to 


$$
\begin{aligned}
\left|C_{F}^{(2)}(t)\right|^{2}= & \frac{1}{\hbar^{2}}\left(\frac{e}{m}\right)^{4}\left(\frac{2 \pi \hbar}{V}\right)^{2} \frac{n_{1}\left(\hat{k}_{1}, \omega_{1}\right) n_{2}\left(\hat{k}_{2}, \omega_{2}\right)}{\omega_{1} \omega_{2} k_{1} k_{2}} \\
& \times\left|P_{f g}^{(2)}\right|^{2} \frac{\sin ^{2} \frac{1}{2}\left[E_{f i} / \hbar-\left(\omega_{1}+\omega_{2}\right)\right] t}{\frac{1}{4}\left[E_{f i} / \hbar-\left(\omega_{1}+\omega_{2}\right)\right]^{2}}
\end{aligned}
$$

$$
P_{f g}^{(2)} \equiv \sum_{m}\left[\frac{\left\langle f\left|\hat{\varepsilon}_{1} \cdot \vec{P}\right| m\right\rangle\left\langle m\left|\hat{\varepsilon}_{2} \cdot \vec{P}\right| g\right\rangle}{E_{m}-E_{g}-\hbar \omega_{2}}+\frac{\left\langle f\left|\hat{\varepsilon}_{2} \cdot \vec{P}\right| m\right\rangle\left\langle m\left|\hat{\varepsilon}_{1} \cdot \vec{P}\right| g\right\rangle}{E_{m}-E_{g}-\hbar \omega_{1}}\right]
$$

Here $|f\rangle,|m\rangle$ and $|g\rangle$ represent electronic states. The transition rate for two-photon absorption is then

$$
\begin{aligned}
& W^{(2)}=\frac{d}{d t} \sum_{F}\left|c_{F}^{(2)}(t)\right|^{2} \\
& \therefore=\frac{1}{\hbar^{2}}\left(\frac{e}{m}\right)^{4}\left(\frac{2 \pi \hbar}{v}\right)^{2} \frac{1}{k_{1} k_{2}} \sum_{f} \frac{d}{d t} \iiint \frac{n_{1}\left(\hat{k}_{1}, \omega_{1}\right) n_{2}\left(\hat{k}_{2}, \omega_{2}\right)}{\omega_{1} \omega_{2}}\left|P_{f g}^{(2)}\right|^{2} \\
& X \frac{\sin ^{2} \frac{1}{2}\left[E_{i g} / \hbar-\left(\omega_{1}+\omega_{2}\right)\right] t}{\frac{1}{4}\left[E_{i g} / \hbar-\left(\omega_{1}+\omega_{2}\right)\right]^{2}} \rho\left(\hbar \omega_{1}\right) \rho\left(\hbar \omega_{2}\right) d\left(\hbar \omega_{1}\right) d\left(\hbar \omega_{2}\right) \text {. }
\end{aligned}
$$

The density of states $\rho\left(\hbar \omega_{i}\right)=d N / d\left(\hbar \omega_{i}\right)$ is given by

$$
\rho\left(\hbar \omega_{i}\right)=\frac{V}{(2 \pi c)^{3}} \frac{\omega_{i}^{2}}{\hbar} d \Omega_{i} \text {. }
$$

Here $d \Omega_{i}=\sin \theta_{i} d \theta_{i} d \phi_{i}$ and $\hat{k}_{i}=\left(\theta_{i}, \phi_{i}\right)$.

$\frac{c}{v} n_{i}\left(\hat{k}_{i}, \omega_{i}\right) \rho\left(\hbar \omega_{i}\right) d\left(\hbar \omega_{i}\right)=\frac{c}{v} n_{i}\left(\hat{k}_{i}, \omega_{i}\right) \frac{V}{(2 \pi c)^{3}} \frac{\omega_{i}^{2}}{\hbar} d\left(\hbar \omega_{i}\right) d \Omega_{i}$

is the number of photons incident per unit area per second having energy within $d\left(\hbar \omega_{i}\right)$ and direction $\hat{k}_{i}$ of propagation within $d \Omega_{i}$. 


$$
f_{i}\left(\omega_{i}\right) d\left(\hbar \omega_{i}\right) \equiv \frac{c}{v} d\left(\hbar \omega_{i}\right) \int \eta_{i}\left(\hat{k}_{i} \omega_{i}\right) \frac{V}{(2 \pi c)^{3}} \frac{\omega_{i}^{2}}{\hbar} d \Omega_{i}
$$

is the number of photons per unit area per second with energy between $\hbar \omega_{i}$ and $\hbar\left(\omega_{i}+d \omega_{i}\right)$.

$$
F_{i} \equiv \int f_{i}\left(\omega_{i}\right) d\left(\hbar \omega_{i}\right)
$$

is the total flux density of beam $i$.

$$
\text { We shall use the fact that } \sin ^{2} x t / x^{2} \text { is strongly }
$$

peaked about $x=0$ for large $t$ by extending the limits of integration over $x$ to $\pm \infty$. In this case

$$
\int_{-\infty}^{\infty} \frac{\sin ^{2} x t}{x^{2}} d x=2 \pi t
$$

We assume that $\left|P_{f g}^{(2)}\right|^{2}$ varies slowly with $\omega_{1}$ and $\omega_{2}$ compared with $f_{1}$ and $f_{2}$. The transition rate then becomes

$W^{(2)}=\frac{2 \pi}{\hbar}\left(\frac{e}{m}\right)^{4}\left(\frac{2 \pi \hbar}{c}\right)^{2} \frac{1}{K_{1} K_{2}} \sum_{f}\left|P_{f g}^{(2)}\right|^{2} \int \frac{f_{1}(\omega) f_{2}\left(E_{f g} / \hbar-\omega\right)}{\omega\left(E_{f g} / \hbar-\omega\right)} d(\hbar \omega)$.

It has been stipulated that $n_{i}(\hat{k}, \omega)$ is a function strongly peaked about $\omega_{i}$, so that $f_{1}$ and $f_{2}$ are narrow bands at $\omega_{1}$ and $\omega_{2}$ respectively, and hence

$$
\int f_{1}(\omega) f_{2}\left(\epsilon_{f g} / \hbar-\omega\right) \alpha \hbar \omega
$$

is a narrow function of $E_{f g}$ centered at $E_{f_{g}}=\hbar \omega_{1}+\hbar \omega_{2}$. Noting that 
15

$$
\iint f_{1}(\omega) f_{2}\left(E_{f g} / \hbar-\omega\right) d(\hbar \omega) d E_{f g}=F_{1} F_{2}
$$

we set

$$
\int f_{1}(\omega) f_{2}\left(E_{\mathrm{fg} / \hbar}-\omega\right) d(\hbar \omega)=F_{1} F_{2} \delta\left[E_{f_{i}}-\hbar\left(\omega_{1}+\omega_{2}\right)\right] .
$$

It follows that

$$
w^{(2)}=\frac{2 \pi}{\hbar}\left(\frac{e}{m}\right)^{4}\left(\frac{2 \pi \hbar}{c}\right)^{2} \frac{F_{1} F_{2}}{\hbar_{1} K_{2} \omega_{1} \omega_{2}} \sum_{f}\left|P_{f g}^{(2)}\right|^{2} \delta\left[E_{f g}-\hbar\left(\omega_{1}+\omega_{2}\right)\right] .
$$

A two-photon absorption coefficient can be defined to describe the attenuation of one of the beams:

$$
\begin{aligned}
\alpha^{(2)} & \equiv \frac{W^{(2)}}{\frac{V F_{1}}{\sqrt{\hbar_{1}}}} \\
& =\frac{(2 \pi)^{3}}{V} \frac{\hbar}{c}\left(\frac{e}{m}\right)^{4} \frac{F_{2}}{\hbar_{2} \sqrt{\pi_{1}} \omega_{1} \omega_{2}} \sum_{f}\left|P_{f g}^{(2)}\right|^{2} \delta\left[E_{f g}-\hbar\left(\omega_{1}+\omega_{2}\right)\right] .
\end{aligned}
$$

$(2-7)$ 
B. Application to Solids

In this section the form of the absorption coefficient will be obtained for electronic transitions in solids in which the ground state is described by a filled valence band and an empty conduction band separated by a forbidden energy gap. Effects of lattice vibrations will be neglected. Two general classifications of two-photon transitions, described as "allowed" and "forbidden" in analogy to Elilot's ${ }^{26}$ classification of one-photon transitions, are discussed in the literature. This terminology is not really appropriate, however, for two reasons: first, it arose in the discussion of inter-band transitions to describe the fact that a "two-photon tran-. sition between bands at a given point in the Brillouin zone was allowed at adjacent points; second, the excited electronic states of a crystal are more correctly described as excitons 2 ? which mix states of different $\vec{k}$, thus formally rendering the classification inappropriate. A distinction between two-photon "allowed" and "forbidden" transitions consistent with the usual terminology, however, is useful, especially when the electron-hole interaction effect is not too large. We will treat, therefore, the two cases defined as follows: let $\left|V\left(\vec{k}_{0}\right)\right\rangle$ and $\left|c\left(\vec{k}_{0}\right)\right\rangle$ be valence and conduction band states at $\vec{k}_{0}$, the point of an extremum of $E_{c}(\vec{k})-E_{v}(\vec{k})$. Then if 


\section{$\sum_{n} \frac{\left\langle c\left(k_{0}\right)|\hat{\varepsilon} \cdot \vec{p}| n\left(k_{0}\right)\right\rangle\left\langle n\left(k_{0}\right)\left|\hat{\varepsilon}_{2} \cdot \vec{p}\right| V\left(k_{0}\right)\right\rangle}{\Omega_{n}}=0$}

for $\Omega_{n}=$ constant, the transition is forbidden. An allowed transition is one which is not forbidden. The most common forbidden transition occurs when $\left|c\left(k_{0}\right)\right\rangle$ and $\left|v\left(k_{0}\right)\right\rangle$ are of opposite parity. In the discussion of forbidden transitions we will assume that this is the case; when some other symmetry property is responsible for the zero in the above equation, the treatment and results are exactly the same.

In order to describe the electronic states of the crystal we first establish the notation. Let $\left|n^{+}(k)\right\rangle$ . label all conduction bands and $\left|\eta^{-}(k)\right\rangle$ all valence bands. Suppose the minimum verticial energy gap occurs at $\vec{k}_{0}$. Then $\left|v_{j}(k)\right\rangle$ and $\left|c_{i}(k)\right\rangle$ are those particular valence and conduction bands, respectively, such that the minimum vertical energy gap is $E_{c_{i}}\left(k_{d}\right)-E_{v_{j}}\left(k_{d}\right)$. That is, $\left\{\left|v_{j}\left(k_{o}\right)\right\rangle\right\}$ is a degenerate set, as is $\left\{\left|c_{i}\left(k_{0}\right)\right\rangle\right\}$.

The ground state of a crystal can be approximated as a determinant of one-electron Bloch states from the valence bands:

$\mathcal{A} \equiv \frac{1}{\sqrt{N !}} \sum_{\mathrm{P}}(-1)^{p} \mathrm{P}$, where p is the parity of permutation P. If the electron-hole interaction is ignored, we can write an excited state as 


$$
\begin{aligned}
|m\rangle \equiv & A \prod_{s=1}^{N}\left|1^{-}\left(k_{s}\right)\right\rangle\left|2^{-}\left(k_{s}\right)\right\rangle \cdots \\
& \cdots\left|n^{-}\left(k_{1}\right)\right\rangle\left|n^{-}\left(k_{2}\right)\right\rangle \cdots\left|n^{+}\left(k^{\prime \prime}\right)\right\rangle \cdots\left|n^{-}\left(k_{N}\right)\right\rangle \cdots \\
\equiv & \left|n^{+}\left(k^{\prime \prime}\right), n^{-}\left(k^{\prime \prime \prime}\right)\right\rangle
\end{aligned}
$$

where valence band state $\left|n^{-}\left(k^{\prime \prime \prime}\right)\right\rangle$ has been replaced by some conduction band state $\left|n^{+}\left(k^{\prime \prime}\right)\right\rangle$.

The final state is

$$
|f\rangle=\left|c_{i}(k), v_{j}\left(k^{\prime}\right)\right\rangle \text {. }
$$

With these states we find

$$
\left\langle m\left|\hat{\varepsilon}_{2} \cdot \vec{p}\right| g\right\rangle
$$

$$
=\left\langle n^{+}\left(k^{\prime \prime}\right)\left|\hat{\varepsilon}_{2} \cdot \vec{p}\right| n^{-}\left(k^{\prime \prime}\right)\right\rangle \delta_{k_{0}^{\prime \prime}, k^{\prime \prime}}
$$

where $\delta_{k}^{\prime \prime}, k^{\prime \prime \prime}$ arises from conservation of momentum.

$$
\left\langle f\left|\hat{\varepsilon}_{1} \cdot \vec{p}\right| m\right\rangle \quad \begin{cases}\delta_{v_{j} n^{-}}\left\langle c_{i}\left(k^{\prime \prime}\right)\left|\hat{\varepsilon}_{1} \cdot \vec{p}\right| n^{\prime \prime}\left(k^{\prime \prime}\right)\right\rangle & \delta^{\prime} \neq k^{\prime \prime \prime} \neq c_{i} \\ -\delta_{c_{i} n^{+}}\left\langle n^{-}\left(k^{\prime \prime \prime}\right)\left|\hat{\varepsilon}_{i} \cdot \vec{p}\right| v_{j}\left(k^{\prime \prime \prime}\right)\right\rangle & n^{-} \neq v_{j} \\ \delta_{v_{j} n^{-}} \delta_{c_{i} n^{+}}+\left\langle c_{i}\left(k^{\prime \prime}\right)\left|\hat{\varepsilon}_{i} \cdot \vec{p}\right| c_{i}\left(k^{\prime \prime}\right)\right\rangle & \\ & \left.-\left\langle v_{j}\left(k^{\prime \prime \prime}\right)\left|\hat{\varepsilon}_{1} \cdot \vec{p}\right| V_{j}\left(k^{\prime \prime \prime}\right)\right\rangle\right]\end{cases}
$$

Substituting into Eq.2-6 we obtain

$$
\begin{aligned}
P_{f g}^{(2)}=\sum_{n}\left[\frac{\left\langle c_{i}(k)\left|\hat{\varepsilon}_{1} \cdot \vec{p}\right| n(k)\right\rangle\left\langle n(k)\left|\hat{\varepsilon}_{2} \cdot \vec{p}\right| V_{j}(k)\right\rangle}{E_{n}(k)-E_{v}(k)-\hbar \omega_{2}}\right. \\
\left.+\frac{\left\langle c_{i}(k)\left|\hat{\varepsilon}_{2} \cdot \vec{p}\right| n(k)\right\rangle\left\langle n(k)\left|\hat{\varepsilon}_{1} \cdot \vec{p}\right| V_{j}(k)\right\rangle}{E_{n}(k)-E_{v}(k)-\hbar \omega_{1}}\right]
\end{aligned}
$$

where $n$ runs over $n^{-}$and $n^{+}$.

Let us consider the case in which the energy difference $E_{c_{i}}(\vec{k})-E_{v_{j}}(\vec{k})$ has a minimum at some point $\vec{k}_{0}$ 
in the Brillouin zone. Near $\vec{k}=\vec{k}_{0}$ state $|n(k)\rangle$ is given by $\vec{k} \cdot \vec{p}$ perturbation theory as ${ }^{28}$ (see Appendix A)

$$
|n(k)\rangle=\left|n\left(k_{0}\right)\right\rangle+\frac{\hbar}{m} \sum_{l}^{\prime} \frac{\left\langle l\left(k_{0}\right)|\vec{k} \cdot \vec{p}| n\left(k_{0}\right)\right\rangle\left|\ell\left(k_{0}\right)\right\rangle}{E_{n}\left(k_{0}\right)-E_{l}\left(k_{0}\right)} .
$$

$\left|c_{i}(k)\right\rangle$ and $\left|V_{j}(k)\right\rangle$ are given by similar expressions.

(Linear terms in the energy sometimes occurring in spinorbit coupled bands will be neglected.) If $\left|c_{i}\left(k_{0}\right)\right\rangle$ and $\left|v_{j}\left(k_{0}\right)\right\rangle$ have the same parity or are of mixed parity, we can neglect the second term in the $\vec{k} \cdot \vec{p}$ expansions:

Allowed

$$
\begin{aligned}
P_{f_{g}}^{(2)}=\sum_{n}\left[\frac{\left\langle c_{i}\left(k_{0}\right)\left|\hat{\varepsilon}_{i} \cdot \vec{p}\right| n\left(k_{0}\right)\right\rangle\left\langle n\left(k_{0}\right)\left|\hat{\varepsilon}_{2} \cdot \vec{p}\right| V_{j}\left(k_{0}\right)\right\rangle}{E_{n}\left(k_{0}\right)-E_{v}\left(k_{0}\right)-\hbar \omega_{2}}\right. \\
\left.+\frac{\left\langle c_{i}\left(k_{0}\right)\left|\hat{\varepsilon}_{2} \cdot \vec{p}\right| n\left(k_{0}\right)\right\rangle\left\langle n\left(k_{0}\right)\left|\hat{\varepsilon}_{1} \cdot \vec{p}\right| V_{j}\left(k_{0}\right)\right\rangle}{E_{n}\left(k_{0}\right)-E_{v}\left(k_{0}\right)-\hbar \omega_{1}}\right]
\end{aligned}
$$

If $\left|c_{i}\left(k_{0}\right)\right\rangle$ and $\left|v_{j}\left(k_{0}\right)\right\rangle$ are of opposite parity, we have:

Forbidden

$$
\begin{aligned}
P_{f g}^{(z)}=\frac{\hbar}{m} \sum_{n}\left[\sum_{l \neq c_{i}} \frac{\left\langle c_{i}|\vec{k} \cdot \vec{p}| l\right\rangle\left\langle\ell\left|\hat{\varepsilon}_{1} \cdot \vec{p}\right| n\right\rangle\left\langle n\left|\hat{\varepsilon}_{2} \cdot \vec{p}\right| v_{j}\right\rangle}{\left(E_{c}-E_{l}\right)\left(E_{n}(k)-E_{v}(k)-\hbar \omega_{2}\right)}\right. \\
\quad+\sum_{l \neq n} \frac{\left\langle c_{i}\left|\hat{\varepsilon}_{1} \cdot \vec{p}\right| \ell\right\rangle\langle l|\vec{k} \cdot \vec{p}| n\rangle\left\langle n\left|\hat{\varepsilon}_{2} \cdot \vec{p}\right| v_{j}\right\rangle}{\left(E_{n}-E_{l}\right)\left(E_{n}(k)-E_{v}(k)-\hbar \omega_{2}\right)} \\
+\sum_{l \neq n} \frac{\left\langle c_{i}\left|\hat{\varepsilon}_{1} \cdot \vec{p}\right| n\right\rangle\langle n|\vec{k} \cdot \vec{p}| \ell\rangle\left\langle\ell\left|\hat{\varepsilon}_{2} \cdot \vec{p}\right| v_{j}\right\rangle}{\left(E_{n}-E_{l}\right)\left(E_{n}(k)-E_{v}(k)-\hbar \omega_{2}\right)} \\
+\sum_{l \neq v} \frac{\left\langle c_{i}\left|\hat{\varepsilon}_{1} \cdot \vec{p}\right| n\right\rangle\left\langle n\left|\hat{\varepsilon}_{2} \cdot \vec{p}\right| \ell\right\rangle\left\langle l|\vec{k} \cdot \vec{p}| v_{j}\right\rangle}{\left(E_{v}-E_{l}\right)\left(E_{n}(k)-E_{v}(k)-\hbar \omega_{2}\right)}
\end{aligned}
$$

+ similar terms with subscripts $I$ and 2 interchanged] 
If the electron-hole interaction is not ignored, the form of the excited states of the crystal must be modified. In this case the crystal Hamiltonian

$$
H=-\frac{\hbar^{2}}{2 m} \sum_{i}\left[\nabla_{i}^{2}+u\left(\vec{r}_{i}\right)\right]+H_{\text {spin }}+\sum_{i<j} \frac{e^{2}}{\left|\vec{r}_{i}-\vec{r}_{j}\right|}
$$

is no longer diagonal with respect to the states $\left|n^{+}(k), n^{-}(k)\right\rangle$. The problem of finding correct linear combinations of these states which diagonalize $\mathrm{H}$ has received considerable attention, and the fruits of its study constitute the theory of excitons ${ }^{27}$. Before returning to the discussion of two-photon absorption we will give a brief description of some of the results of exciton theory.

It is a good approximation for weakly bound excitons to construct the states from two bands, but we will retain more than two when several are degenerate at $\vec{k}=\vec{k}_{0}$. Thus, for example, we can write an excited crystal state

$$
|c v, \mu \vec{K}\rangle=\sum_{k, i, j} F_{c_{i} v_{j}}^{\mu}(\vec{k}, \vec{k}-\vec{K})\left|c_{i}(\vec{k}), v_{j}(\vec{k}-\vec{K})\right\rangle
$$
where $F_{c_{i} v_{j}}(\vec{k}, \vec{k}-\vec{K})$ satisfies

$$
\sum_{k^{\prime}, i^{\prime}, j^{\prime}}\left\langle c_{i}(k), v_{j}(k-K)|H| c_{i^{\prime}}\left(k^{\prime}\right), v_{j^{\prime}}\left(k^{\prime}-K\right)\right\rangle F_{c_{i i^{\prime} j^{\prime}}^{\mu}}^{\mu}\left(k^{\prime} k^{\prime}-K\right)=E_{c v k}^{\mu} F_{c_{i} v_{j}}^{\mu}(k, k-K)
$$

$\vec{K}$ is the exciton momentum, the difference between the electron and hole momenta, and is one of the labels of the space group representation according to which $|c v, \mu \vec{k}\rangle$ transforms. Since the ground state and $\overrightarrow{\mathrm{P}}$ transform 
according to $\overrightarrow{\mathrm{K}}=0$ representations, the intermediate states and the final state must also. This is simply another way of saying that crystal momentum is conserved. We will therefore need only $\vec{K}=0$ excitons, so we define.

$$
f_{c_{i} v_{j}}^{\mu}(\vec{k}) \equiv F_{c_{i} v_{j}}^{\mu}(\vec{k}, \vec{k}) \text {. }
$$

Then $f$ must satisfy

$$
\sum_{k^{\prime} i^{\prime} j^{\prime}}\left\langle c_{i}(k), v_{j}(k)|H| c_{i^{\prime}}\left(k^{\prime}\right), v_{j},\left(k^{\prime}\right)\right\rangle f_{c_{i}, v_{j^{\prime}}}^{\mu}\left(k^{\prime}\right)=E_{c v_{0}}^{\mu} f_{c_{i} v_{j}}^{\mu}(\vec{k})
$$

One can define the Fourier transform of $f$,

$$
U_{c_{i} v_{i}}^{\mu}(\vec{\beta}) \equiv N^{-\frac{1}{2}} \sum_{k} e^{i \vec{k} \cdot \vec{\beta}} f_{c_{i} v_{j}}^{\mu}(\vec{k})
$$

where $\vec{\beta}$ is the separation of the localized (Wannier) electron state lattice site and the hole state lattice site. These localized states $a_{n R}(\vec{r})$ are defined by the (unitary) Fourier transform of Bloch functions

$$
a_{n k}(\vec{r}) \equiv a_{n}(\vec{r}-\vec{R})=\frac{1}{\sqrt{N}} \sum_{k} e^{-i \vec{k} \cdot \vec{R}} \psi_{n k}(\vec{r})
$$

where $\vec{R}$ is a lattice site: The symmetry properties and degree of localization of $a_{n R}(\vec{r})$ about $\vec{R}$ are discussed by Slater, ${ }^{29}$ Koster 30 and Kohn. $31 \quad u_{c_{i} v_{j}}^{\mu}(\vec{\beta})$ is commonly known as the envelope function.

In the case of two non-degenerate bands Eq.2-II can be written

$$
\sum_{k^{\prime}}\left\langle c(k), v(k)|H| c\left(k^{\prime}\right), v\left(k^{\prime}\right)\right\rangle f_{c v}^{\mu}\left(k^{\prime}\right)=E_{c v}^{\mu} f_{c v}^{\mu}(k)
$$

or 
22

$$
\begin{gathered}
\left\{\left\langle c(k)\left|H^{e}\right| c(k)\right\rangle-\left\langle v(k)\left|H^{h}\right| v(k)\right\rangle\right\} \\
\quad-\sum_{k^{\prime}}\left\langle c(k), v(k)\left|\frac{e^{2}}{\left|\vec{r}_{e}-\vec{r}_{h}\right|}\right| c\left(k^{\prime}\right), v\left(k^{\prime}\right)\right\rangle f_{c v}^{\mu}\left(k^{\prime}\right) \\
\quad=\left[E_{c v}^{\mu}-E_{0}-E_{g a p}\right] f_{c v}^{\mu}\left(k^{\prime}\right)
\end{gathered}
$$

where $\mathrm{E}_{0}$ is the crystal ground state energy and $E_{\text {gap }}=E_{c}\left(k_{0}\right)-E_{v}\left(k_{0}\right)$. When the electron-hole interaction is weak we expect the electron-hole separation to be, on the average, large, so that $f(k)$ is a narrow function centered at $\vec{k}_{0}$. We can therefore write Eq.2-13 in an - effective mass approximation as

$$
\begin{gathered}
\frac{\hbar^{2}}{2}\left[\sum_{\lambda \lambda^{\prime}}\left(\frac{1}{m_{c}^{*}}-\frac{1}{m_{v}^{*}}\right)_{\lambda \lambda^{\prime}}\left(k-k_{0}\right)_{\lambda}\left(k-k_{0}\right)_{\lambda^{\prime}}\right] f_{c v}^{\mu}(k) \\
-\sum_{k^{\prime}}\left\langle c(k)_{0} v(k)\left|\frac{e^{2}}{\left|\vec{r}_{e}-\vec{r}_{h}\right|}\right| c\left(k^{\prime}\right), v\left(k^{\prime}\right)\right\rangle f_{c v}^{\mu}\left(k^{\prime}\right) \\
=\left[E_{c v}^{\mu}-E_{g a p}-E_{0}\right] f_{c v}^{\mu}\left(k^{\prime}\right)
\end{gathered}
$$

In the case of spherical non-degenerate bands Wanner 32 showed that Eq.2-14. leads to the hydrogenic equation

$$
\left[-\frac{\hbar^{2}}{2 \mu} \nabla_{\beta}^{2}-\frac{e^{2}}{\beta}\right] u_{c v}^{\mu}(\vec{\beta})=\left[E_{c v}^{\mu}-E_{0}-E_{g a p}\right] u_{c v}^{\mu}(\vec{\beta})
$$

Here the separation $\left|\vec{r}_{e}-\vec{r}_{h}\right|$ was, on the average, large, and it could be replaced by the separation of the electron and hole lattice sites $\vec{\beta}=\left|\vec{R}_{e}-\vec{R}_{h}\right| \approx\left|\vec{r}_{e}-\vec{r}_{h}\right|$. Since the electron-hole interaction varied slowly from lattice site 
to lattice site, $\vec{\beta}$ is approximated to be a continuous variable. The energies of the two bands (assumed here to have extrema at $\vec{k}=0$ ) near $\vec{k}=0$ were given in the effective mass approximation by

$$
E_{\alpha}(\vec{k})=E_{\alpha}(0)+\frac{\hbar^{2} k^{2}}{2 m_{\alpha}^{*}}
$$

$1 / \mu \equiv 1 / m_{c}^{*}-1 / m_{v}^{*}$ is the reduced inverse effective mass. Corrections which introduce a dielectric constant into the hydrogenic equation are discussed in the book by Knox. 27

The effective mass approximation is good for largeradius excitons, since in this case $f_{c v}^{\mu}(k)$ is a narrow function centered at $\vec{k}=0$. This approximation is not suitable, however, when the electron and hole are strongly interacting.

When an extremum of $E_{c}(\vec{k})-E_{v}(\vec{k})$ occurs at a point other than $\vec{k}=0$, or in anisotropic crystals at $\vec{k}=0$, one obtains from Eq.2-14 an equation somewhat more difficult than Eq.2-15:

$$
\begin{array}{r}
\left\{-\frac{\hbar^{2}}{2} \sum_{\lambda \lambda^{\prime}}\left(\frac{1}{m_{c}^{*}}-\frac{1}{m_{r}^{*}}\right)_{\lambda \lambda^{\prime}} \frac{\partial^{2}}{\partial \beta_{\lambda} \partial \beta_{\lambda^{\prime}}}-\frac{e^{2}}{\beta}\right\} u(\vec{\beta}) \\
=\left[E-E_{0}-E_{\text {gap }}\right] U(\vec{\beta})
\end{array}
$$

It is worthwhile to note that the operator in braces is invariant under operations of the point group of the crystal, so that $U(\vec{\beta})$ (and $f(k)$ ) must transform according to a representation of the point group. 
In the case of degeneracy at band extrema, the matrix $\left\langle c_{i}(k), v_{j}(k)|H| C_{i^{\prime}}\left(k^{\prime}\right) v_{j^{\prime}}\left(k^{\prime}\right)\right\rangle$ can be written

$$
H_{i i^{\prime}}^{e} \delta_{j j^{\prime}} \delta_{k k^{\prime}}+H_{j j^{\prime}}^{h} \delta_{i i^{\prime}} \delta_{k k^{\prime}}+V_{i j k, i^{\prime} j^{\prime} k^{\prime}}^{e h}
$$

where

$$
H^{e}=-\frac{\hbar^{2}}{2 m} \nabla_{r_{e}}^{2}+u_{e}\left(\vec{r}_{e}\right), \quad H^{h}=-\frac{\hbar^{2}}{2 m} \nabla_{r_{h}}^{2}+u_{h}\left(\vec{r}_{h}\right)
$$

and

$$
V^{e h}=-\frac{e^{2}}{\left|\vec{r}_{e}-\vec{r}_{h}\right|} \text {. }
$$

Dresselhaus $33_{\text {has }}$ treated the case of large radius excitons, in which case $V_{i j k, i^{\prime} j^{\prime}, k^{\prime}}^{\text {eh }}=V_{k, k^{\prime}}^{\text {eh }} \delta_{i i^{\prime}} \delta_{j j^{\prime}}$ and, in an effective mass approximation,

where

$$
\begin{aligned}
& H_{i i^{\prime}}^{e}=\frac{\hbar^{2}}{2} \sum_{\alpha \beta}\left(\frac{1}{m_{e}^{* \prime}}\right)_{i i^{\prime}}^{\alpha \beta}\left(k_{e}-k_{e}^{0}\right)_{\alpha}\left(k_{e}-k_{e}^{0}\right)_{\beta}+E_{c}\left(k_{0}\right) \delta_{i i^{\prime}} \\
& H_{j j^{\prime}}^{h}=\frac{\hbar^{2}}{2} \sum_{\alpha \beta}\left(\frac{1}{m_{h^{*}}^{*}}\right)_{j j^{\prime}}^{\alpha \beta}\left(k_{h}-k_{h}^{0}\right)_{\alpha}\left(k_{h}-k_{h^{\prime}}^{0}\right)_{\beta}+E_{v}\left(k_{0}\right) \delta_{j j^{\prime}}
\end{aligned}
$$

$$
\left(\frac{1}{m_{e}^{*}}\right)_{\left\{i^{\prime}\right.}^{\alpha \beta} \equiv \frac{1}{m} \delta_{i i^{\prime}} \delta_{\alpha \beta}+\frac{1}{m^{2}} \sum_{l}^{\prime}\left[\frac{\left\langle c_{i}\left|p_{\alpha}\right| l\right\rangle\left\langle l\left|p_{\beta}\right| c_{i^{\prime}}\right\rangle}{E_{c}-E_{l}}+\frac{\left\langle c_{i}\left|p_{\beta}\right| l\right\rangle\left\langle l\left|p_{\alpha}\right| c_{i^{\prime}}\right\rangle}{E_{c}-E_{l}}\right]
$$
etc.

If we had chosen initially the correct band states, $\mathrm{H}^{e}$ and $\mathrm{H}^{h}$ would be diagonal and the problem would reduce to several problems of two bands each. However, in this case the effective masses are functions of the direction in $\vec{k}$ space away from the extrema. An example of this will be given in Chapter IV. This warping of bands has an effect on the type of equation which must be solved for the coefficients f. Under these circumstances, Eq.2-14 cannot be reduced to an equation as simple as even Eq.2-16. One finds 
$\frac{\hbar^{2}}{2} \sum_{\beta^{\prime}} G_{c_{i} v_{j}}\left(\vec{\beta}-\vec{\beta}^{\prime}\right) U_{c_{i} v_{j}}^{\mu}\left(\vec{\beta}^{\prime}\right)-\frac{e^{2}}{\beta} U_{c_{i} v_{j}}^{\mu}(\vec{\beta})=\left[E_{c_{i} v_{j}}^{\mu}-E_{o}-E_{g a p}\right] U_{c_{i} v_{j}}^{\mu}(\vec{\beta})$

where

$$
G_{c_{i} v_{j}}\left(\vec{\beta}-\vec{\beta}^{\prime}\right) \equiv N^{-1} \sum_{k} e^{i \vec{k} \cdot\left(\vec{\beta}-\vec{\beta}^{\prime}\right)} \sum_{\lambda \lambda^{\prime}}\left[\frac{1}{\mu_{i j}\left(k_{\theta}, k_{\phi}\right)}\right]_{\lambda \lambda^{\prime}} k_{\lambda} k_{\lambda^{\prime}}
$$

- Again note that since $\sum_{\lambda x^{\circ}}\left[V \mu\left(k_{\theta} k_{\phi}\right)\right]_{\lambda x^{\prime}} k_{\lambda} k_{\lambda^{\prime}}$ is totally symmetric with respect to the point group, U must transform under a representation of the point group.

In cases where the warping of the bands is slight, i. e.,

$$
\left[\frac{1}{\mu_{i j}\left(k_{0} k_{\phi}\right)}\right]_{\lambda \lambda^{\prime}}=\left[\frac{1}{\mu_{i j}(0,0)}\right]_{\lambda \lambda^{\prime}}+\delta g\left(k_{\theta} k_{\phi}\right)_{\lambda \lambda^{\prime}}
$$

where $\delta$ is small, one might first solve for zero-order wave functions by Eq.2-15 or Eq.2-16, and then treat the "integral operator".

$$
\delta \frac{\hbar^{2}}{2} \sum_{\beta^{\prime}} N^{-1} \sum_{k} e^{i \vec{k} \cdot\left(\vec{\beta}-\vec{\beta}^{\prime}\right)} \sum_{\lambda \lambda^{\prime}} g\left(k_{\Delta} k_{\phi}\right)_{\lambda x^{\prime}} k_{\lambda} k_{\lambda^{\prime}}
$$

as a perturbation.

The correct states to use in the second-order matrix element $P_{f g}^{(2)}$, both in the final state and in the intermediate states, are the stationary states of the crystal, that is, exciton states. Elliot ${ }^{26}$ and Dresselhaus 34 have given the form of the absorption coefficient for single-photon transitions to exciton states. For the two-photon absorption coefficient we will assume that we can work with excitons constructed from two bands. The 
final state is

$$
\left|c_{i} v_{j} ; \mu\right\rangle=\sum_{k} f_{c_{i} v_{j}}^{\mu}(k)\left|c_{i}(k), v_{j}(k)\right\rangle
$$

and a typical intermediate state is

$$
\left|n^{+} n^{-} ; \nu\right\rangle=\sum_{k} f_{n^{+} n^{-}}^{\nu}(k)\left|n^{+}(k), n^{-}(k)\right\rangle .
$$

.. The ground state to intermediate state matrix element of $P_{f g}^{(2)}$ is

$$
\left\langle m\left|\hat{\varepsilon}_{2} \cdot \vec{P}\right| g\right\rangle=\sum_{k} f_{n^{+}}^{\nu}(k)^{*}\left\langle n^{+}(k)\left|\hat{\varepsilon}_{2} \cdot \vec{p}\right| n^{-}(k)\right\rangle
$$

The intermediate state to final state matrix element is $\left\langle f\left|\hat{\varepsilon}_{1} \cdot \vec{p}\right| m\right\rangle$

$$
=\left\{\begin{array}{cc}
\delta_{v_{j} n^{-}} \sum_{k^{\prime}} f_{c_{i} v_{j}}^{\mu}\left(k^{\prime}\right)^{*} f_{n^{+} v_{j}}^{\nu}\left(k^{\prime}\right)\left\langle c_{i}\left(k^{\prime}\right)\left|\hat{\varepsilon}_{i} \cdot \vec{p}\right| n^{+}\left(k^{\prime}\right)\right\rangle & n^{+} \neq c_{i} \\
-\delta_{c_{i} n^{+}} \sum_{k^{\prime}} f_{c_{i} v_{j}}^{\mu}\left(k^{\prime}\right)^{*} f_{c_{i} n^{-}}^{\nu}\left(k^{\prime}\right)\left\langle n^{-}\left(k^{\prime}\right)|\hat{\varepsilon} \cdot \vec{p}| v_{j}\left(k^{\prime}\right)\right\rangle & n^{-} \neq v_{j} \\
\delta_{v_{j} n^{-}}-\delta_{c_{i} n^{+}} \sum_{k^{\prime}} f_{c_{i} v_{j}}^{\mu}\left(k^{\prime}\right)^{*} f_{c_{i} v_{j}}\left(k^{\prime}\right) \\
x\left[\left\langle c_{i}\left(k^{\prime}\right)|\hat{\varepsilon} \cdot \vec{p}| c_{i}\left(k^{\prime}\right)\right\rangle-\left\langle v_{j}\left(k^{\prime}\right)|\hat{\varepsilon}, \vec{p}| v_{j}\left(k^{\prime}\right)\right\rangle\right]
\end{array}\right.
$$

Unless the conduction and valence band are of opposite parity, the first term in the $\vec{k} \bullet \vec{p}$ expansions gives the dominant contribution. Then Eq. 2-17 becomes

$$
\begin{aligned}
\left\langle m\left|\hat{\varepsilon}_{i} \cdot \vec{p}\right| g\right\rangle & =\left\langle n^{+}\left(k_{0}\right)\left|\hat{\varepsilon}_{2} \cdot \vec{p}\right| n^{-}\left(k_{0}\right)\right\rangle \sum_{k} f_{n^{+} n^{-}}^{\nu}(\vec{k})^{*} \\
& =\left\langle n^{+}\left(k_{0}\right)\left|\hat{\varepsilon}_{2} \cdot \vec{p}\right| n^{-}\left(k_{0}\right)\right\rangle N^{\frac{1}{2}} U_{n^{+} n^{-}}^{\nu}(0)^{*}
\end{aligned}
$$

where the band extrema occur at $\vec{k}_{0} \cdot U_{n^{+} n^{-}}^{\nu}(\vec{\beta})$ must therefore have the total symmetry of the point group. If parity is well defined there are other possible terms arising from 
Eq.2-17. If the valence and conduction bands have the same parity, then intermediate states of the same parity can contribute by keeping the cross terms of $\left\langle n^{+}(k)\left|\hat{\varepsilon}_{2} \cdot \hat{p}\right| n^{-}(k)\right\rangle$ when $\vec{k} \cdot \vec{p}$ expansions of $\left|n^{+}(k)\right\rangle$ and $\left|n^{-}(k)\right\rangle$ are supplied. In this case Eq.2-17 becomes

$$
\begin{aligned}
\left\langle m\left|\hat{\varepsilon}_{2} \cdot \vec{P}\right| g\right\rangle= & \sum_{k} f_{n^{+} n^{-}}^{\nu}(k)^{*} \frac{\hbar}{m} \sum_{l}^{\prime}\left\{\frac{\left\langle n^{+}\left(k_{0}\right)\left|\hat{\varepsilon}_{2} \cdot \vec{p}\right| l\left(k_{0}\right)\right\rangle\left\langle l\left(k_{0}\right)|\vec{k} \cdot \vec{p}| n^{-}\left(k_{0}\right)\right\rangle}{E_{n^{-}}\left(k_{0}\right)-E_{l}\left(k_{0}\right)}\right. \\
& \left.+\frac{\left\langle n^{+}\left(k_{0}\right)|\vec{k} \cdot \vec{p}| l\left(k_{0}\right)\right\rangle\left\langle l\left(k_{0}\right) \hat{\varepsilon}_{2} \cdot \vec{p} \mid n^{-}\left(k_{0}\right)\right\rangle}{E_{n^{-}}\left(k_{0}\right)-E_{l}\left(k_{0}\right)}\right\} \\
= & \sum_{k} f_{n^{+} n^{-}}^{\nu}(k)^{*} \hat{\varepsilon}_{2} \cdot \overleftrightarrow{M}_{n^{+} n^{-}} \cdot \hbar \vec{k}
\end{aligned}
$$

where

$$
M_{n^{+} n^{-}}^{\alpha \beta} \equiv \frac{1}{m} \sum_{l}^{\prime}\left\{\frac{\left\langle n^{+}\left|p_{\alpha}\right| \ell\right\rangle\left\langle l\left|p_{\beta}\right| n^{-}\right\rangle}{E_{n^{-}}-E_{l}}+\frac{\left\langle n^{+}\left|p_{\beta}\right| \ell\right\rangle\left\langle l\left|p_{\alpha}\right| n^{-}\right\rangle}{E_{n^{+}}-E_{l}}\right\} .
$$

$\frac{1}{m} \vec{M}_{n^{+} n^{-}}$is an "inverse interband effective mass" tensor which occurs in one-photon dipole forbidden transitions 35 and one-photon quadrupole allowed transitions. 36

$$
\begin{aligned}
& \left\langle m\left|\hat{\varepsilon}_{2} \cdot \vec{P}\right| g\right\rangle=N^{-\frac{1}{2}} \sum_{k, \beta} U_{n^{+} n^{-}}^{\nu}(\beta)^{*} \hat{\varepsilon}_{z} \cdot \overleftrightarrow{M}_{n^{+} n^{-}} \cdot \hbar \vec{k} e^{i \vec{k} \cdot \vec{\beta}} \\
& \cong \frac{N^{\frac{1}{2}}}{(2 \pi)^{3}} \iint d^{3} \beta d^{3} k u_{n^{+} n^{-}}^{\nu}(\beta)^{*} \hat{\varepsilon}_{2} \cdot \overleftrightarrow{M}_{n^{+} n^{-}} \cdot \frac{\hbar}{i} \nabla_{\beta} e^{i \vec{k} \cdot \vec{\beta}} \\
& =N^{\frac{1}{2}} \int \alpha^{3} U_{n^{+}{ }^{-}}^{\nu}(\beta)^{*} \hat{\varepsilon}_{2} \cdot \vec{M}_{n^{+} n^{-}} \cdot \frac{\hbar}{i} \nabla_{\beta} \delta(\vec{\beta}) \\
& =\left.N^{\frac{1}{2}} \hat{\varepsilon}_{2} \cdot \overleftrightarrow{M}_{n^{+} n^{-}} \cdot \frac{\hbar}{i} \nabla_{\beta} u_{n^{*} n^{-}}^{\nu}(\beta)^{*}\right|_{\beta=0}(2-20)
\end{aligned}
$$

For hydrogenic excitons $U_{n^{+} n}^{\nu}-(\vec{\beta})$ must be a $p$-state. This contribution. to "allowed" excitation will be ignored for the present, but it will be returned to in Chapter III. Eq. 2-18 becomes 
$\left\langle f\left|\hat{\varepsilon}_{1} \cdot \vec{P}\right| m\right\rangle$

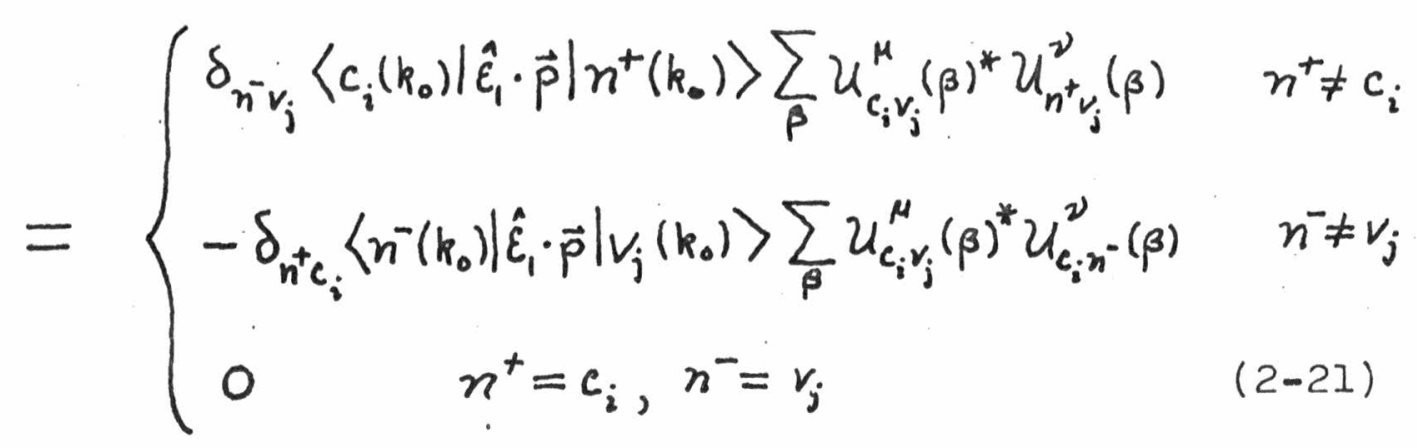

It is seen that $U^{\mathcal{N}}$ must belong to the same representation as $U^{\mu}$.

Substitution of Eq.2-19 and Eq.2-21 into Eq.2-6 yields

Allowed

$$
\begin{aligned}
& P_{f g}^{(2)}=N^{\frac{1}{2}} \sum_{\nu \beta}\left\{\sum_{n^{+}} U_{c_{i} v_{j}}^{\mu}(\beta)^{*} U_{n^{+} v_{j}}^{\nu}(\beta) U_{n^{+} v_{j}}^{\nu}(0)^{*}\right. \\
& \times\left[\frac{\left\langle c_{i}\left(k_{0}\right)\left|\hat{\varepsilon}_{i} \cdot \vec{p}\right| n^{+}\left(k_{0}\right)\right\rangle\left\langle n^{+}\left(k_{0}\right)\left|\hat{\varepsilon}_{2} \cdot \vec{p}\right| V_{j}\left(k_{0}\right)\right\rangle}{E_{n^{+} V_{j}}(\nu)-\hbar \omega_{2}}\right] \\
& -\sum_{n^{-}} U_{c_{i} v_{j}}^{\mu}(\beta)^{*} U_{c_{i} \eta^{-}}^{\nu}(\beta) U_{c_{i} \eta^{-}}^{\nu}(0)^{*} \\
& X\left[\frac{\left\langle c_{i}\left(k_{0}\right)\left|\hat{\varepsilon}_{i} \cdot \vec{p}\right| n^{-}\left(k_{0}\right)\right\rangle\left\langle n^{-}\left(k_{0}\right)\left|\hat{\varepsilon}_{i} \cdot \vec{p}\right| v_{j}\left(k_{0}\right)\right\rangle}{E_{c_{j} n^{-}}(\nu)-\hbar \omega_{2}}\right] \\
& + \text { terms with } 1 \text { and } 2 \text { interchanged }
\end{aligned}
$$

The ground state energy is taken to be zero.

When the valence and conduction bands have opposite parity we must keep both parities of intermediate states. When Eq.2-19 applies, Eq.2-18 becomes 
29

$$
\begin{aligned}
& \left\langle f\left|\hat{\varepsilon}_{1} \cdot \vec{P}\right| m\right\rangle=\sum_{k} f_{c_{i} v_{j}}^{\mu}(k)^{*} f_{n^{+} v_{j}}^{\nu}(k) \\
& x \frac{\hbar}{m} \sum_{l}^{\prime}\left\{\frac{\left\langle c_{i}\left(k_{0}\right)\left|\hat{\varepsilon}_{i} \cdot \vec{p}\right| l\left(k_{0}\right)\right\rangle\left\langle l\left(k_{0}\right)|\vec{k} \cdot \vec{p}| n^{+}\left(k_{0}\right)\right\rangle}{E_{n^{+}}-E_{l}}\right. \\
& \left.+\frac{\left\langle c_{i}\left(k_{0}\right)|\vec{k} \cdot \vec{p}| \ell\left(k_{0}\right)\right\rangle\left\langle\ell\left(k_{0}\right)\left|\hat{\varepsilon_{i}} \cdot \vec{p}\right| n^{+}\left(k_{0}\right)\right\rangle}{E_{c}-E_{l}}\right\}
\end{aligned}
$$

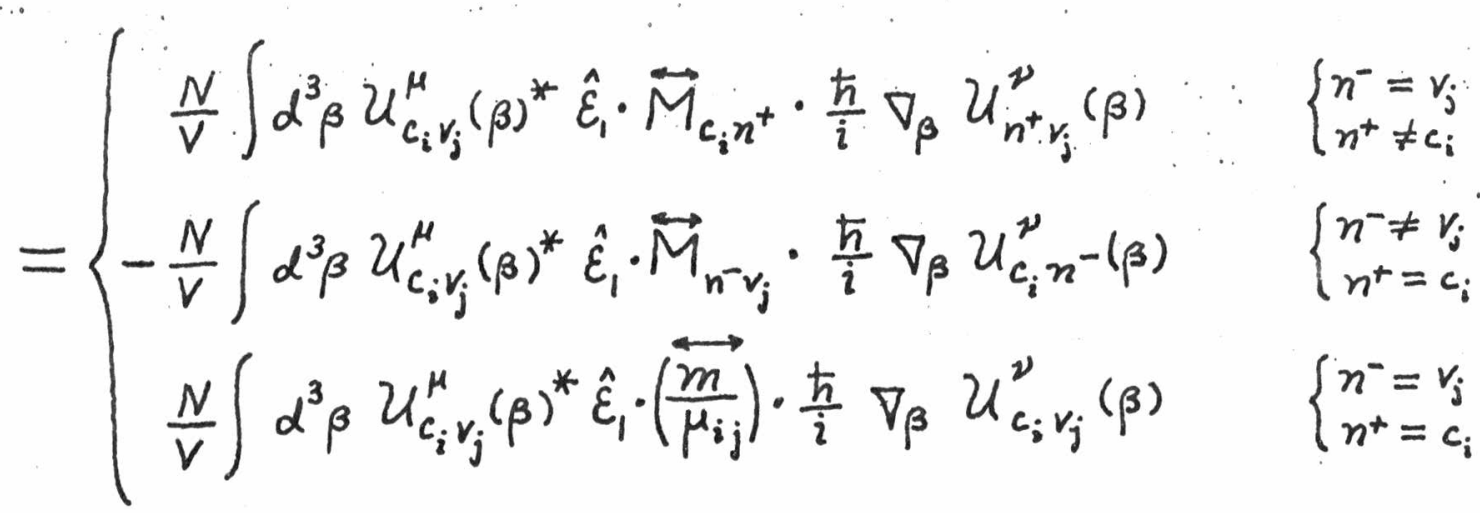

$$
\begin{aligned}
& \left(\frac{1}{\mu_{i j}}\right)=\frac{1}{m} \overleftrightarrow{M}_{c_{i} c_{i}}-\frac{1}{m} \overleftrightarrow{M}_{v_{j} v_{j}}
\end{aligned}
$$

is the reduced reciprocal effective mass tensor.

When Eq.2-20 applies for $\left\langle m\left|\hat{\varepsilon}_{k} \cdot \vec{p}\right| g\right\rangle$, Eq.2-2I gives $\left\langle f\left|\hat{\varepsilon}_{1} \cdot \vec{p}\right| m\right\rangle$. With these matrix elements we have

Forbidden

$$
\begin{aligned}
& P_{f g}^{(x)}= \\
& \left\{N ^ { \frac { 1 } { 2 } } \sum _ { \nu } \left[\sum_{n^{+}} U_{n^{+} v_{j}}^{\nu}(0)^{*} \frac{\hat{\varepsilon}_{i} \cdot \overleftrightarrow{M}_{c_{i} n^{+}} \cdot\left\langle U_{c_{i} v_{j}}^{\mu}|\vec{p}| u_{n^{+} v_{j}}^{\nu}\right\rangle}{E_{n^{+} v_{j}}(\nu)-\hbar \omega_{2}}\left\langle n^{+}\left(k_{0}\right)\left|\hat{\varepsilon}_{p} \cdot \vec{p}\right| v_{j}\left(k_{0}\right)\right\rangle\right.\right. \\
& \left.-\sum_{n^{-}} u_{c_{i} n^{-1}}^{\nu}(0)^{*} \frac{\hat{\varepsilon}_{1} \cdot \vec{M}_{n^{-} v_{i}} \cdot\left\langle u_{c_{i} v_{j}}^{\mu}|\vec{p}| u_{c_{i} n^{n}}^{\nu}\right\rangle}{E_{c_{i} n^{-}}(\nu)-\hbar \omega_{2}}\left\langle c_{i}\left(k_{0}\right)\left|\hat{\varepsilon}_{i} \cdot \vec{p}\right| \eta^{-}\left(k_{0}\right)\right\rangle\right]
\end{aligned}
$$




$$
\begin{aligned}
& +N^{\frac{1}{2}} \sum_{\nu} \sum_{n^{+}}\left\langle c_{i}\left(k_{0}\right)|\hat{\varepsilon} \cdot \vec{p}| n^{+}\left(k_{0}\right)\right\rangle \frac{\left.\left\langle u_{c i v_{j}}^{\mu} \mid u_{n^{+} v_{j}}^{\nu}\right\rangle \hat{\varepsilon}_{2} \cdot \vec{M}_{n^{+} v_{j}} \cdot \frac{\hbar}{i} \nabla_{\beta} u_{n^{+} v_{j}}^{\nu}(\beta)\right|_{0}}{E_{n^{+} v_{j}}(\nu)-\hbar \omega_{2}} \\
& -\sum_{n^{-}}\left\langle n^{-}\left(k_{0}\right)\left|\hat{\varepsilon_{i}} \cdot \vec{p}\right| V_{j}\left(k_{0}\right)\right\rangle \frac{\left.\left\langle U_{c_{i} v_{j}}^{\mu} \mid U_{c_{i} n^{-}}\right\rangle \hat{\varepsilon}_{i^{\prime}} \cdot \vec{M}_{c_{i} n^{-}} \cdot \frac{\hbar}{i} \nabla_{\beta} U_{c_{i} n^{-}}^{\nu}(\beta)\right|_{0}}{E_{c_{i} n^{-}}(\nu)-\hbar \omega_{2}} \\
& + \text { terms with subscripts } 1 \text { and } 2 \text { interchanged }
\end{aligned}
$$

Here

$$
\left\langle u_{c_{i} v_{j}}^{\mu}|\vec{p}| u_{n^{+} v_{j}}^{\nu}\right\rangle \equiv \frac{N}{V} \int U_{c_{i} v_{j}}^{\mu}(\beta)^{*} \frac{\hbar}{i} \nabla_{\beta} u_{n^{+} v_{j}}^{\nu}(\beta) d^{3} \beta \quad(2-24)
$$

and

$$
\left\langle u_{c_{i} v_{j}}^{\mu} \mid u_{n^{+} v_{j}}^{\nu}\right\rangle \equiv \frac{N}{v} \int u_{c_{i} v_{j}}^{\mu}(\beta)^{*} u_{n^{+} v_{j}}^{\nu}(\beta) d^{3} \beta .
$$

Let us now consider the significance of the terms in Eq.2-23. Each line represents a class of paths followed in the two-photon absorption process. Fig.2-I is a schematic diagram of these paths with $(a),(b),(c)$ and (d) indicating representatives of paths corresponding to the first, second, third and fourth lines, respectively, of Eq.2-23. In Fig.2-1 the vertical lines represent the initial, intermediate and final states as indicated at the top of each diagram. The horizontal dashes label conduction and valence band states. The parity of the bands is indicated by $g$ or $u$. (When we speak of the parity of a band we will mean the parity of the band at the extremum.) Circles and crosses represent holes and. 
$|g\rangle|m\rangle|f\rangle|g\rangle|m\rangle|f\rangle \quad|g\rangle|m\rangle|f\rangle \quad|g\rangle|m\rangle|f\rangle$

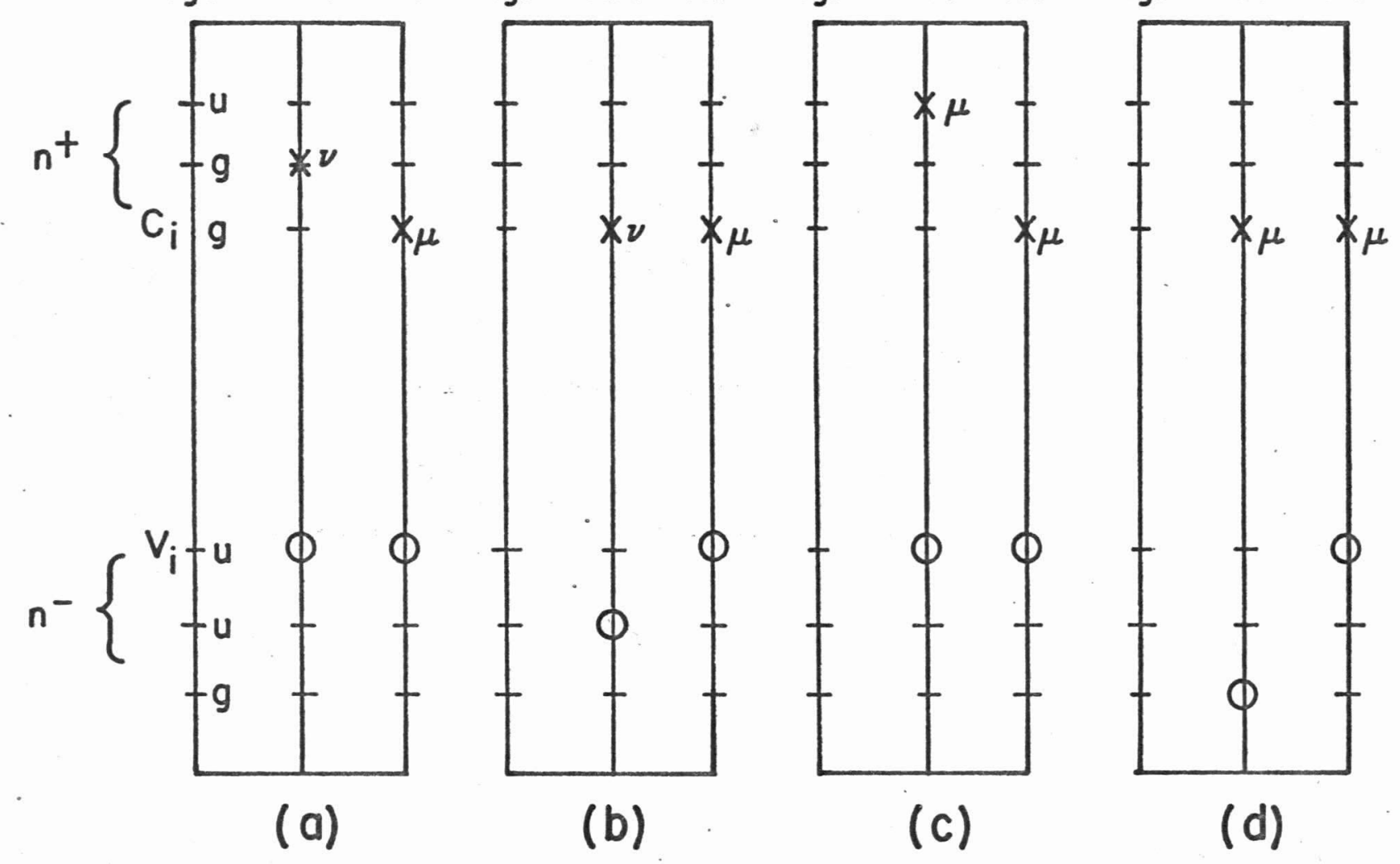

Fig.2-1. Paths of forbidden two-photon absorption 
electrons, respectively, and $p$ and $\mu$ label envelope functions of the electron-hole pair. Note that $\nu$ is always totally symmetric and, in a cubic crystal, $\mu$ must have $T_{1 u}$ symmetry

Referring again to Eq.2-23, the first line represents a path in which a photon raises an electron from the valence band $v_{j}$ to a conduction band of opposite parity, the position of the electron with respect to the hole being described by a totally symmetric envelope. The other photon forces the electron to the final state with the electron in band $c_{i}$, its position with respect to the hole then given by envelope function $\nu$. The second line describes the path in which the final state is reached via the intermediate state in which an electron in band $c_{i}$ moves, with respect to a hole in a valence band of parity opposite to that of $\mathbf{c}_{i}$, according to a totally symmetric envelope. In the third line the intermediate state consists of an electron in an upper conduction band having the same parity as $v_{j}$, the band of the hole, the envelope function having the same symmetry as that of the final state. The envelope also has the same symmetry in the intermediate state of the fourth line, where the electron in band $c_{i}$ moves with respect to the hole in a lower valence band having parity the same as the conduction band. Notice that the final exciton envelope function $U_{c_{i} v_{j}}^{\mu}$ in all terms must be a p-state in the 
hydrogenic model.

In this section we have given analytic expressions for the second-order matrix element $\mathrm{P}_{\mathrm{fg}}^{(2)}$, and hence the absorption coefficient by Eq.2-7, for allowed and forbidden transitions both in the simple band approximation and in an approximation to include exciton effects. To conclude this chapter we will give a brief description of relevant theoretical work which has appeared in the literature. Braunstein ${ }^{37}$ and Braunstein and Ockman ${ }^{38}$ have given the form of the two-photon absorption coefficient in a three-band model neglecting exciton effects. They have characterized transitions by the behavior of the matrix elements $\left\langle n\left(k_{0}\right)|\hat{\varepsilon} \cdot \vec{p}| v\left(k_{0}\right)\right\rangle$ and $\left\langle c\left(k_{0}\right)|\hat{\varepsilon} \cdot \vec{p}| n\left(k_{0}\right)\right\rangle$, "allowedforbidden", for example, meaning that $\left\langle n\left(k_{0}\right)|\hat{\varepsilon} \cdot \vec{p}| v\left(k_{0}\right)\right\rangle \neq 0$ and $\left\langle c\left(k_{0}\right)|\hat{\varepsilon} \cdot \vec{p}| n\left(k_{0}\right)\right\rangle=0$. Both their allowed-allowed case. and forbidden-forbidden case correspond to our allowed case. $P_{f g}^{(2)}$ in this model is obtained from Eq.2-8 by retaining a single intermediate band $n$, different from $v$ and $c$ and keeping the terms of order $l, k$, and $k^{2}$ for allowed-allowed, allowed-forbidden, and forbiddenforbidden, respectively. The first two are special cases of Eq.2-9 and Eq.2-10. We have assumed in deriving Eq.2-9 that when $\left|c\left(k_{0}\right)\right\rangle$ and $\left|v\left(k_{0}\right)\right\rangle$ have the same parity there will be opposite parity intermediate states $\left|n\left(k_{0}\right)\right\rangle$ whose contribution to $\mathrm{P}_{\mathrm{fg}}^{(\boldsymbol{k})}$ is much larger than the con- 
tribution of order $k^{2}$ of the same parity intermediate states.

Loudon ${ }^{39}$ and Inoue and Toyozawall have given the form of the two-photon absorption coefficient in the allowed case when the final states are excitons, but have neglected to use excitons in the intermediate states. Their result for $P_{f g}^{(2)}$ was

$$
\begin{aligned}
P_{f g}^{(z)}=u_{c_{i} v_{i}}^{\mu}(0)^{*} \sum_{n} & \left\{\frac{\left\langle c_{i}\left(k_{0}\right)\left|\hat{\varepsilon}_{i} \cdot \vec{p}\right| n\left(k_{0}\right)\right\rangle\left\langle n\left(k_{0}\right)\left|\hat{\varepsilon}_{2} \cdot \vec{p}\right| v_{j}\left(k_{0}\right)\right\rangle}{E_{n}\left(k_{0}\right)-E_{v}\left(k_{0}\right)-\hbar \omega_{2}}\right. \\
& \left.+\frac{\left\langle c_{i}\left(k_{0}\right)\left|\hat{\varepsilon}_{2} \cdot \vec{p}\right| n\left(k_{0}\right)\right\rangle\left\langle n\left(k_{0}\right)\left|\hat{\varepsilon}_{1} \cdot \vec{p}\right| v_{j}\left(k_{0}\right)\right\rangle}{E_{n}\left(k_{0}\right)-E_{v}\left(k_{0}\right)-\hbar \omega_{1}}\right\}
\end{aligned}
$$

We find a similar expression for the forbidden case:

$$
\begin{aligned}
P_{f g}^{(2)}= & N^{\frac{1}{2}} \sum_{n} \frac{\left.\hat{\varepsilon}_{1} \cdot \vec{M}_{c_{i} n} \cdot \frac{\hbar}{i} \nabla_{\beta} u_{c i v_{j}}^{\mu}(\beta)^{*}\right|_{0}\left\langle n\left(k_{0}\right)\left|\hat{\varepsilon}_{2} \cdot \vec{p}\right| v_{j}\left(k_{0}\right)\right\rangle}{E_{n}\left(k_{0}\right)-E_{r}\left(k_{0}\right)-\hbar \omega_{2}} \\
& +N^{\frac{1}{2}} \sum_{n} \frac{\left.\left\langle c_{i}\left(k_{0}\right)\left|\hat{\varepsilon}_{1} \cdot \vec{p}\right| n\left(k_{0}\right)\right\rangle \hat{\varepsilon}_{2} \cdot \vec{M}_{n v_{i}} \cdot \frac{\hbar}{i} \nabla_{\beta} u_{c_{i} v_{j}}^{\mu}(\beta)\right|_{0}}{E_{n}\left(k_{0}\right)-E_{v}\left(k_{0}\right)-\hbar \omega_{2}} \\
& + \text { terms with subscripts } 1 \text { and } 2 \text { interchanged }
\end{aligned}
$$

We will make no more use of this expression. Mahan ${ }^{19,20}$ has calculated the form of the absorption coefficient for the allowed and forbidden cases in a twoband model, using excitons in the final and intermediate states. His calculations will be discussed in Chapter IV。 


\section{Chapter III}

\section{Allowed Transitions}

The usefulness of studying the dependence of the two-photon absorption coefficient on the directions of polarization of the incident light beams was first pointed out by Inoue and Toyozawa, 11 who calculated the angular dependence functions for a transition in which the initial state had the total symmetry of the symmetry group. When considered from the point of view of Bloch functions, one might ask what the polarization dependence is for a transition between a valence band and a conduction band for an arbitrary symmetry of either. In this chapter we will derive a mathematical expression for the polarization dependence of such a transition at the $\Gamma$-point and tabulate the angular dependences as a supplement to the tables of Inoue and Toyozawa. In addition we will obtain angular functions for transitions taking place at point's and on lines of high symmetry in the Brillouin zone for hexagonal close-packed and wurtzite structures. 
.36

A. Formalism

Let us first consider the sum over intermediate states in Eq.2-22. Here we must make some simplifying assumptions. If several bands are degenerate at $\vec{k}=\vec{k}_{0}$, we will assume that the difference in the effective masses of these bands at $\vec{k}_{0}$ is sufficiently small that

$$
N^{\frac{1}{2}} \sum_{\nu \beta} \frac{u_{c_{i} v_{j}}^{\mu}(\vec{\beta})^{*} u_{n_{l}^{+} v_{j}}^{\nu}(\vec{\beta}) u_{n_{l}^{+} v_{j}}^{\nu}(0)^{*}}{E_{n_{l}^{+} v_{j}^{(\nu)}}-\hbar \omega}
$$

and

$$
N^{\frac{1}{2}} \sum_{\nu \beta} \frac{U_{c_{i} v_{j}}^{\mu}(\vec{\beta})^{*} U_{c_{i} n_{l}^{-}}^{\nu}(\vec{\beta}) U_{c_{i} n_{l}^{-}}^{\nu}(0)^{*}}{E_{c_{i} n_{l}^{-}}(\nu)-\hbar \omega}
$$

are approximately independent of $i, j$ and $l$, where $\left\{\left|C_{i}\left(k_{0}\right)\right\rangle\right\},\left\{\left|v_{j}\left(k_{0}\right)\right\rangle\right\}$ and $\left\{\left|n_{l}^{ \pm}\left(k_{0}\right)\right\rangle\right\}$ are degenerate sets. The second-order matrix element, excluding or including exciton effects, can then be written

$$
\begin{aligned}
p_{f g}^{(2)}=\left\langle c_{i}\left(k_{0}\right)\left|\hat{\varepsilon}_{1} \cdot \vec{p} \Lambda\left(\omega_{2}\right) \vec{p} \cdot \hat{\varepsilon}_{2}\right| v_{j}\left(k_{0}\right)\right\rangle \\
\\
+\left\langle c_{i}\left(k_{0}\right)\left|\hat{\varepsilon}_{2} \cdot \vec{p} \Lambda\left(\omega_{1}\right) \hat{\mathcal{E}}_{1} \cdot \vec{p}\right| v_{j}\left(k_{0}\right)\right\rangle
\end{aligned}
$$

where we define 
37

$$
\begin{aligned}
& \Lambda\left(\omega_{m}\right)= \\
& \begin{cases}\sum_{n} \frac{\left|n\left(k_{0}\right)\right\rangle\left\langle n\left(k_{0}\right)\right|}{E_{n}\left(k_{0}\right)-E_{v}\left(k_{0}\right)-\hbar \omega_{m}} & \text { in Eq.2-9 or Eq .2-25 } \\
N^{\frac{1}{2}} \sum_{n^{+}} \sum_{\nu \beta} U_{c v}^{\mu}(\vec{\beta})^{*} U_{n^{+} v}^{\nu}(\vec{\beta}) U_{n^{+} v}^{\nu}(0)^{*} & \frac{\left|n^{+}\left(k_{0}\right)\right\rangle\left\langle n^{+}\left(k_{0}\right)\right|}{E_{n^{+} v}(\nu)-\hbar \omega_{m}} \\
+N^{\frac{1}{2}} \sum_{n^{-}} \sum_{p \beta} U_{c v}^{\mu}(\vec{\beta})^{*} U_{c n^{-}}^{\nu}(\vec{\beta}) U_{c n^{-}}^{\nu}(0)^{*} \frac{\left|n^{-}\left(k_{0}\right)\right\rangle\left\langle n^{-}\left(k_{0}\right)\right|}{E_{c n^{-}}(\nu)-\hbar \omega_{m}} & \text { in Eq.2-22 }\end{cases}
\end{aligned}
$$

$\bigwedge\left(\omega_{m}\right)$ has the total symmetry of the group of $\vec{k}_{0}$ (see Appendix B). Following Inoue and Toyozawa ${ }^{\text {ll }}$ we define

$$
\begin{aligned}
& \Lambda_{+}=\Lambda\left(\omega_{2}\right)+\Lambda\left(\omega_{1}\right) \\
& \Lambda_{-}=\Lambda\left(\omega_{2}\right)-\Lambda\left(\omega_{1}\right) .
\end{aligned}
$$

The two-photon absorption coefficient is proportional to

$$
\sum_{f}\left|p_{f g}^{(2)}\right|^{2}=\sum_{k_{0} i j}\left|\left\langle c_{i}\left(k_{0}^{\prime}\right)\left|\left[\hat{\varepsilon}_{1} \cdot \vec{p} \wedge\left(\omega_{2}\right) \vec{p} \cdot \hat{\varepsilon}_{2}+\hat{\varepsilon}_{2} \cdot \vec{p} \wedge\left(\omega_{1}\right) \vec{p} \cdot \hat{\varepsilon}_{1}\right]\right| v_{j}\left(k_{0}^{\prime}\right)\right\rangle\right|^{2}
$$

where $\vec{k}_{0}^{\prime}$ runs over the star $\vec{k}_{0} .40$

Using Eqs.3-1 the operator in square brackets becomes

$$
\begin{aligned}
& \frac{1}{2} \hat{\varepsilon}_{1} \cdot \vec{p}\left(\Lambda_{+}+\Lambda_{-}\right) \vec{p} \cdot \hat{\varepsilon}_{2}+\frac{1}{2} \hat{\varepsilon}_{2} \cdot \vec{p}\left(\Lambda_{+}-\Lambda_{-}\right) \vec{p} \cdot \hat{\varepsilon}_{1} \\
& =\frac{1}{2}\left\{\hat{\varepsilon}_{1} \cdot \vec{p} \Lambda_{+} \vec{p} \cdot \hat{\varepsilon}_{2}+\hat{\varepsilon}_{2} \cdot \vec{p} \Lambda_{+} \vec{p} \cdot \hat{\varepsilon}_{1}\right\} \\
& +\frac{1}{2}\left\{\hat{\varepsilon}_{1} \cdot \vec{p} \Lambda_{-} \vec{p} \hat{\varepsilon}_{2}-\hat{\varepsilon}_{2} \cdot \vec{p} \Lambda_{-} \vec{p} \cdot \hat{\varepsilon}_{1}\right\}
\end{aligned}
$$


38

$=\hat{\varepsilon}_{1} \cdot\left\{\left(\vec{p} \wedge_{+} \vec{p}\right)_{S}+\left(\vec{p} \Lambda_{-} \vec{p}\right)_{A S}\right\} \cdot \hat{\varepsilon}_{2}$

where $S$ and AS mean the symmetric and antisymmetric parts. The tensor in braces, which will be labeled $\vec{T}$, can be written in matrix form as

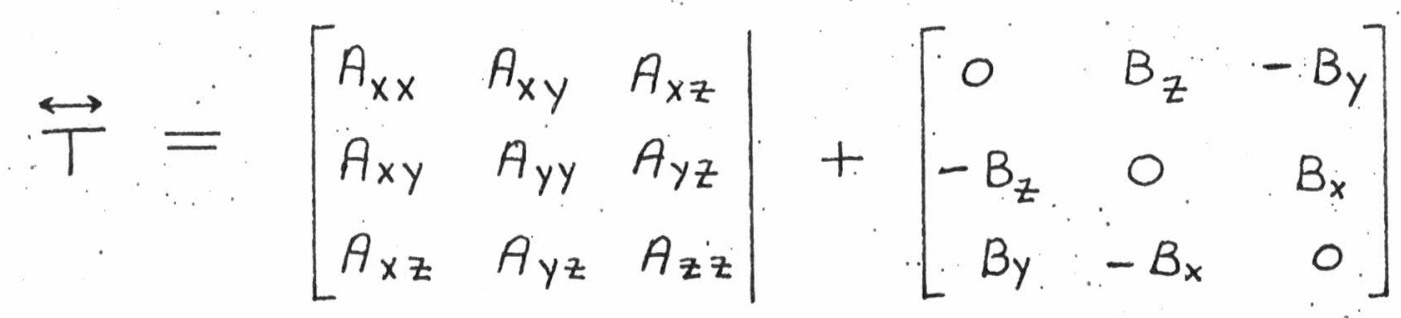

Here

$$
\begin{aligned}
& B_{x} \equiv \frac{1}{2}\left(p_{y} \Lambda_{-} p_{z}-p_{z} \Lambda_{-} p_{y}\right) \\
& B_{y} \equiv \frac{1}{2}\left(p_{z} \Lambda_{-} p_{x}-p_{x} \wedge_{-} p_{z}\right) \\
& B_{z} \equiv \frac{1}{2}\left(p_{x} \Lambda_{-} p_{y}-p_{y} \Lambda_{-} p_{x}\right)
\end{aligned}
$$

transform as components of an axial vector (or pseudovector), and

$$
A_{i j} \equiv \frac{1}{2}\left(p_{i} \wedge_{+} p_{j}+p_{j} \wedge_{+} p_{i}\right)
$$

transform as the product $i j$ (e. g., A $x y$ transforms as $x y)$. Recalling the notation for the polarization vectors, $\hat{\varepsilon}_{1}=\left(l_{1}, m_{1}, n_{1}\right)$ and $\varepsilon_{2}=\left(l_{2}, m_{2}, n_{2}\right)$, the tensor operator is written in expanded form as

$$
\begin{aligned}
& \hat{\varepsilon}_{1} \cdot \stackrel{\leftrightarrow}{T} \cdot \hat{\varepsilon}_{2}=l_{1} l_{2} A_{x x}+m_{1} m_{2} A_{y y}+n_{1} n_{2} A_{z z} \\
& +\left(n_{1} l_{2}+n_{2} l_{1}\right) A_{x z}+\left(m_{1} n_{2}+m_{2} n_{1}\right) A_{y z}+\left(l_{1} m_{2}+l_{2} m_{1}\right) A_{x y} \\
& +\left(n_{1} l_{2}-n_{2} l_{1}\right) B_{y}+\left(m_{1} n_{2}-m_{2} n_{1}\right) B_{x}+\left(l_{1} m_{2}-l_{2} m_{1}\right) B_{z}
\end{aligned}
$$


For a given point group each term in this equation will not necessarily transform under an irreducible representation of the point group. For example, $A_{x x}$ does not transform within a single representation of the cubic group, but $A_{x x}+A_{y y}+A_{z z}$ transforms according to the representation A, of group 0 . Therefore the terms in Eq.3-2 must be rearranged so as to be a sum of terms which transform according to specific rows of irreducible representations of the given point group; i. e.,

$$
\hat{\varepsilon}_{1} \cdot \ddot{T} \cdot \hat{\varepsilon}_{2}=\sum_{\mu n q} a_{n q}^{\mu} T_{n q}^{\mu}
$$

where $T_{n q}^{\mu}$ transforms according to row $n$ of representation $\Gamma^{\mu}$. The index $q$ distinguishes terms belonging to the same row of the same representation if more than one term. occurs in the decomposition of $\hat{\varepsilon}_{1} \cdot \overleftrightarrow{T} \cdot \hat{\varepsilon}_{2}$. The coefficients $a_{n q}^{\mu}$ are functions of $\hat{\varepsilon}_{1}$ and $\hat{\varepsilon}_{2}$. (Henceforth representations will be labeled by Greek letters and rows by Latin letters.)

Let us relabel the valence and conduction band states in order to make their symmetry explicit; $\left|c_{i}\left(k_{0}\right)\right\rangle \equiv\left|\lambda_{i}\right\rangle$ and $\left|v_{j}\left(k_{0}\right)\right\rangle \equiv\left|\nu_{j}\right\rangle$, where $\lambda$ and $\nu$ are irreducible representations of the group of $\vec{k}_{0}$, and $i$ and $j$ are rows. The absorption coefficient is now proportional to

$$
\cdot \sum_{k_{0} i j}\left|\left\langle\lambda i\left|\hat{\varepsilon}_{1} \cdot \stackrel{T}{T} \cdot \hat{\varepsilon}_{2}\right| \nu_{j}\right\rangle\right|^{2}=\sum_{k_{0}^{\prime} i j}\left|\sum_{\mu n q} a_{n q}^{\mu}\left\langle\lambda i\left|T_{n q}^{\mu}\right| \nu_{j}\right\rangle\right|^{2} .
$$


The following mathematical theorem will be proven. If an operator $T$ can be decomposed into a sum of terms $T_{n q}^{\mu}$ which transform according to rows $n$ of representation $\Gamma^{\mu}$ of a group, i. e., $T=\sum_{\mu n q} a_{n q}^{\mu} T_{n q}^{\mu}$, then

$$
\sum_{i j}\left|\sum_{\mu n q} a_{n q}^{\mu}\left\langle\lambda i\left|T_{n q}^{\mu}\right| \nu_{j}\right\rangle\right|^{2}=\sum_{\mu \tau_{\mu}} \gamma\left(\mu, \tau_{\mu}\right),(3-3)
$$

where the sum over $\mu$ on the right extends only over those representations contained in the direct product $\Gamma^{\lambda *} \times \Gamma^{\nu}$, and

$$
\gamma\left(\mu, \tau_{\mu}\right) \equiv \sum_{s}\left|\left\langle\Gamma^{\prime}\left|\sum_{\sigma n q} a_{n q}^{\sigma} T_{n q}^{\sigma}\right| \mu \tau_{\mu} s\right\rangle\right|^{2} .
$$

$\Gamma^{\prime}$ is the totally symmetric representation, and $\tau_{\mu}$ distinguishes occurrences of $\Gamma^{\mu}$ if it occurs more than once in the decomposition of $\Gamma^{\lambda *} x \Gamma^{\nu}$. The theorem says that the angular function for a transition between states of symmetries $\Gamma^{\lambda}$ and $\Gamma^{\nu}$ is a linear combination of angular functions characteristic of transitions of the type $\Gamma^{\prime} \rightarrow \Gamma^{\mu}$ for all $\Gamma^{\mu}$. contained in the decomposition of $\Gamma^{\lambda^{*}} \times \Gamma^{\nu}$, and that the function for a transition of the type $\Gamma^{\prime} \rightarrow \Gamma^{\mu}$ will occur as many times as $\Gamma^{\mu}$ is contained in $\Gamma^{\lambda *} \times \Gamma^{\nu}$ Eq.3-4 can be written

$$
\begin{aligned}
\gamma\left(\mu, \tau_{\mu}\right) & =\sum_{s}\left|\sum_{\sigma n q} a_{n q}^{\sigma}\left\langle\Gamma^{\prime}\left|\sum_{\rho \tau_{\rho} r}\left[\begin{array}{c}
\rho \tau_{\rho}, \sigma \mu \\
r, \sigma_{s}
\end{array}\right]^{*}\right| \rho \tau_{\rho} r ; \tau_{\mu q}\right\rangle\right| \\
& =\sum_{s}\left|\sum_{q} a_{n q}^{\mu} \xi_{q}^{\mu \tau_{\mu}}\right|^{2},
\end{aligned}
$$

where ${ }^{4 I}$ we have used the fact that $\left\langle\Gamma^{\prime} \mid \rho \tau_{\rho} r ; \tau_{\mu} g\right\rangle=0$ 
41

unless $\Gamma^{p}=\Gamma^{l}$, in which case $\Gamma^{\sigma}$ must be equal to $\Gamma^{M}$ $\xi_{q}^{\mu \tau_{\mu}}$ are complex constants, and $A_{r}^{e} \tau_{p},{ }_{n s} \mu$ are the Clebsch-Gordan coefficients defined by

$$
\Psi_{r}^{\rho \tau_{\rho}}=\sum_{n s}\left[\begin{array}{c}
\rho \tau_{\rho}, \sigma \mu \\
r, n_{s}
\end{array}\right] \psi_{n}^{\sigma} \phi_{s}^{\mu} .
$$

To prove the theorem we write

$$
\begin{aligned}
& \sum_{i j}\left|\sum_{\mu n q} a_{n q}^{\mu}\left\langle\lambda i\left|T_{n q}^{\mu}\right| \nu j\right\rangle\right|^{2} \\
& =\sum_{i j}\left|\sum_{\mu n q} a_{n q}^{\mu} \sum_{\sigma \tau_{\sigma} s}\left[A^{\sigma \tau_{\sigma}, \mu \nu} \begin{array}{c}
\mu \nu \\
s
\end{array}\right]^{*}\left\langle\lambda i \mid \sigma \tau_{\sigma} s ; i\right\rangle\right|^{2} \\
& =\sum_{i j}\left|\sum_{\mu n q} a_{n q}^{\mu} \sum_{\tau_{\lambda}}\left[\begin{array}{cc}
\lambda \tau_{\lambda}, & \mu \nu \\
i & n j
\end{array}\right]^{*} \eta_{q}^{\tau_{\lambda}}\right|^{2},
\end{aligned}
$$

where $\eta_{q}^{\tau_{\lambda}}$ are complex constants. At this point we use a symmetry property of Clebsch-Gordan coefficients derived in Appendix $\mathrm{C}$ :

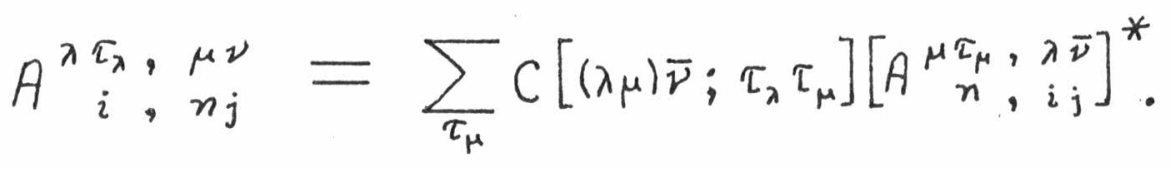

$C\left[(\lambda \mu) \bar{\nu} ; \tau_{\lambda} \tau_{\mu}\right]$ is a complex constant, and $\bar{\nu}$ denotes the representation which is complex conjugate to representation $\nu$. Continuing, Eq.3-6 becomes

$$
\sum_{i j}\left|\sum_{\mu n q} a_{n q}^{\mu} \sum_{\tau_{\lambda} \tau_{\mu}} C\left[(\lambda \mu) \bar{\nu}_{;} \tau_{\lambda} \tau_{\mu}\right]^{*}\left[\begin{array}{c}
\mu \tau_{\mu}, \lambda \bar{\nu} \\
n, i j
\end{array}\right] \eta_{q} \tau_{\lambda}\right|^{2}
$$




$$
\begin{aligned}
=\sum_{i j} \sum_{\mu n q} & \sum_{\mu^{\prime} n^{\prime} q^{\prime}} \sum_{\tau_{\lambda} \tau_{\mu}} \sum_{\tau_{\lambda^{\prime}} \tau_{\mu^{\prime}}}\left(a_{n q}^{\mu}\right)\left(a_{n^{\prime} q^{\prime}}^{\mu^{\prime}}\right)^{*} \\
& \times C\left[(\lambda \mu) \bar{\nu} ; \tau_{\lambda} \tau_{\mu}\right]^{*} C\left[\left(\lambda \mu^{\prime}\right) \bar{\nu} ; \tau_{\lambda^{\prime}} \tau_{\mu^{\prime}}\right]\left(\eta_{q}^{\tau_{\lambda}}\right)\left(\eta_{q^{\prime}}^{\tau_{\lambda^{\prime}}}\right)^{*} \\
& \times\left[\begin{array}{c}
A \tau_{\mu}, \lambda \bar{\nu} \\
n, i j
\end{array}\right]\left[\begin{array}{c}
A^{\mu^{\prime}} \tau_{\mu^{\prime}}, \lambda \bar{\nu} \\
n^{\prime}, i j
\end{array}\right] .
\end{aligned}
$$

The Clebsch-Gordan coefficients are elements of a unitary matrix, so that when the sum over $i$ and $j$ is performed (see Appendix C) we obtain

$$
\begin{aligned}
\sum_{i j} & \left|\sum_{\mu n q} a_{n q}^{\mu}\left\langle\lambda i\left|T_{n q}^{\mu}\right| \nu_{j}\right\rangle\right|^{2} \\
& =\sum_{\mu \tau_{\mu} n}\left|\sum_{q} a_{n q}^{\mu} \sum_{\tau_{\lambda}} C\left[(\lambda \mu) \bar{\nu} ; \tau_{\lambda} \tau_{\mu}\right]^{*} \eta_{q}^{\tau_{\lambda}}\right|^{2} \\
& =\sum_{\mu \tau_{\mu} n}\left|\sum_{q} a_{n q}^{\mu} \xi_{q}^{\mu \tau_{\mu}}\right|^{2} \\
& =\sum_{\mu \tau_{\mu}} \gamma\left(\mu, \tau_{\mu}\right),
\end{aligned}
$$

which completes the proof.

Before proceeding to the angular functions for the point groups, some comments must be made about the paths followed for allowed two-photon absorption. The preceding formalism was based on the assumption that only bands having parity opposite to that of the highest valence and lowest conduction bands entered into the intermediate states. This is an approximation, of course; all bands participate to some degree. Mahan ${ }^{20}$ has considered 
allowed two-photon absorption as being well-described within the framework of the two band model, thereby requiring that the bands in the intermediate states have the same parity as the valence and conduction bands. By proceeding with Eq.2-20. with $\mathrm{n}^{+}=\mathrm{c}$ and $\mathrm{n}^{-}=\mathrm{v}$, Mahan showed that final state excitons could be s- or d-states, and indicated that the s-states had been considered earlier by Inoue and Toyozawa. 11 It is apparent, however, that the s-states considered by Inoue and Toyozawa are reached by different paths. The transitions of Inoue and. Toyozawa are analogous to the allowed-allowed transitions of Braunstein and Ockman, 37,38 while Mahan's transitions are analogous to the forbidden-forbidden type. our assumption, then, is that the probability amplitudes for s-exciton transitions via opposite parity intermediate bands are considerably larger than the probability amplitudes for s- and d-exciton transitions described in the two-band model. 


\section{B. $\Gamma$-point Transitions}

The angular dependence functions for all possible band-to-band transitions occurring at $\vec{k}=0$ and to exictons of $\Gamma^{\prime}$ symmetry (when the stated assumptions are valid) are given in Table 3-1, where $\lambda_{i}$ are real constants and $c_{i}$ are real positive constants. As we have said before, transitions are not allowed between states of opposite parity, and so we have written $\Gamma_{ \pm}^{\mu} \longleftrightarrow \Gamma_{ \pm}^{\nu}$, meaning $\Gamma_{-}^{\mu} \longleftrightarrow \Gamma_{-}^{\nu}$ or $\Gamma_{+}^{\mu} \longleftrightarrow \Gamma_{+}^{\nu}$, when the point group contains the inversion. When two representations are given in parentheses they are degenerate by time reversal. 41 The symbols used for the single-valued representations are those common in molecular applications ( $A, B, E$ and $T$ ) and the symbols used for the double-valued representations $\left(\Gamma^{\mu}\right)$ are defined in the book by Koster, Dimmock, Wheeler and statz. 42 To minimize the possibility of confusion in the notation, Appendix $D$ provides a table of correspondence between the notation used here and the notation of Koster, et al. The definitions of the axes used for some of the point groups are given in Fig.3-I through Fig. 3-4. For the remaining groups $-C_{n}, S_{n}$ and $C_{n h}$-the $z$ axis is the axis of rotation, and $x$ and $y$ are arbitrary. Note that for some groups one can make an unambiguous choice of axes, while for others (e.g. $D_{3 d}$ ) one must be more careful, for the angular functions for these are not invariant under a coordinate rotation of $90^{\circ}$. 
In compiling Table 3-1 the angular functions for transitions of the type $\Gamma^{\prime} \longleftrightarrow \Gamma^{\mu}$ have been recalculated and agree with those reported by Inoue and Toyozawa except for the following cases: transitions of the type $\Gamma^{\prime} \longrightarrow \Gamma^{E}$, where E denotes a pair of complex conjugate representations, for the groups $\mathrm{C}_{4}, \mathrm{C}_{4 h}, \mathrm{~S}_{4}, \mathrm{C}_{3}, \mathrm{~S}_{6}, \mathrm{C}_{6}, \mathrm{C}_{6 \mathrm{~h}}$ and $\mathrm{C}_{3 \mathrm{~h}}$. The disagreement appears to be due to an algebraic error in the earlier calculation.

It is important to make some comments concerning the application of the angular functions in Table 3-1 in experiments. First, we point out the restriction placed on the directions of polarization made in part $A$ of Chapter II. Second, it must be noted that $\Lambda_{+}$and $\Lambda_{-}$are functions of $\omega_{1}$ and $\omega_{2}$; when $\omega_{1}=\omega_{2}, \bigwedge_{\text {_ }}$ is zero. Of course, this must be avoided in a two-beam experiment, as was stated in Chapter II. However, if the two frequencies are close, the two-photon absorption coefficient and polarization dependence can be strongly dependent on the frequency of the tunable light source. In the tables this fact is hidden in the constants; one needs only to remember that the components (and only the components) of $\left(\hat{\varepsilon}_{1} \times \hat{\varepsilon}_{2}\right), e \cdot g$. $\left(I_{1} m_{2}-I_{2} m_{1}\right)$, have coefficients dependent on $\Lambda_{-}$. 
Table 3-1

Angular Dependence Functions

The group(s) are given at the heads of the left hand columns. Other symbols are defined in the text.

\begin{tabular}{|c|c|c|}
\hline $\mathrm{C}_{1}$ & $\mathrm{~S}_{2}$ & - \\
\hline $\begin{array}{l}A \leftrightarrow A \\
\Gamma^{2} \leftrightarrow \Gamma^{2}\end{array}$ & $\begin{array}{l}\mathrm{A}_{ \pm} \leftrightarrow \mathrm{A}_{ \pm} \\
\Gamma_{ \pm}^{2} \leftrightarrow \Gamma_{ \pm}^{2}\end{array}$ & $\begin{aligned} {\left[I_{1} I_{2}\right.} & +\lambda_{1} m_{1} m_{2}+\lambda_{2} n_{1} n_{2}+\lambda_{3}\left(I_{1} m_{2}+I_{2} m_{1}\right)+\lambda_{4}\left(n_{1} I_{2}+n_{2} I_{1}\right) \\
& +\lambda_{5}\left(m_{1} n_{2}+m_{2} n_{1}\right)+\lambda_{6}\left(I_{1} m_{2}-I_{2} m_{1}\right)+\lambda_{7}\left(n_{1} I_{2}-n_{2} I_{1}\right) \\
& \left.+\lambda_{8}\left(m_{1} n_{2}-m_{2} n_{1}\right)\right]^{2}\end{aligned}$ \\
\hline
\end{tabular}

\begin{tabular}{|c|c|c|c|}
\hline $\mathrm{C}_{2}$ & ${ }^{\circ} C_{I h}$ & $\mathrm{C}_{2 \mathrm{~h}}$ & \\
\hline $\begin{array}{l}A \leftrightarrow A \\
B \leftrightarrow B\end{array}$ & $\begin{array}{l}A^{\prime} \leftrightarrow A^{\prime} \\
A^{\prime \prime} \leftrightarrow A^{\prime \prime}\end{array}$ & $\begin{array}{l}\mathrm{A}_{ \pm} \leftrightarrow \mathrm{A}_{ \pm} \\
\mathrm{B}_{ \pm} \leftrightarrow \mathrm{B}_{ \pm}\end{array}$ & $\begin{aligned} {\left[I_{1} I_{2}+\lambda_{1} m_{1} m_{2}+\lambda_{2} n_{1} n_{2}\right.} & +\lambda_{3}\left(I_{1} m_{2}+I_{2} m_{1}\right) \\
& \left.+\lambda_{4}\left(I_{1} m_{2}-I_{2} m_{1}\right)\right]^{2} \equiv A\left(\varepsilon_{1} \varepsilon_{2}\right)\end{aligned}$ \\
\hline$A \leftrightarrow B$ & $A^{\prime} \Leftrightarrow A^{\prime \prime}$ & $A_{ \pm} \leftrightarrow B_{ \pm}$ & $\begin{aligned} {\left[\left(m_{1} n_{2}-m_{2} n_{1}\right)+\lambda_{5}\left(n_{1} l_{2}\right.\right.} & \left.-n_{2} I_{1}\right)+\lambda_{6}\left(n_{1} I_{2}+n_{2} l_{1}\right) \\
& \left.+\lambda_{7}\left(m_{1} n_{2}+m_{2} n_{1}\right)\right]^{2} \equiv B\left(\varepsilon_{1} \varepsilon_{2}\right)\end{aligned}$ \\
\hline$\left(\Gamma^{3} \Gamma^{4}\right) \leftrightarrow$ & )$\leftrightarrow\left(\Gamma^{3} \Gamma^{4}\right.$ & $\left.\Phi_{ \pm}^{4}\right) \infty\left(\Gamma_{ \pm}^{3} \Gamma_{ \pm}^{4}\right)$ & $A\left(\varepsilon_{1} \varepsilon_{2}\right)+c B\left(\varepsilon_{1} \varepsilon_{2}\right)$ \\
\hline
\end{tabular}


Table 3-1 (Contd.)

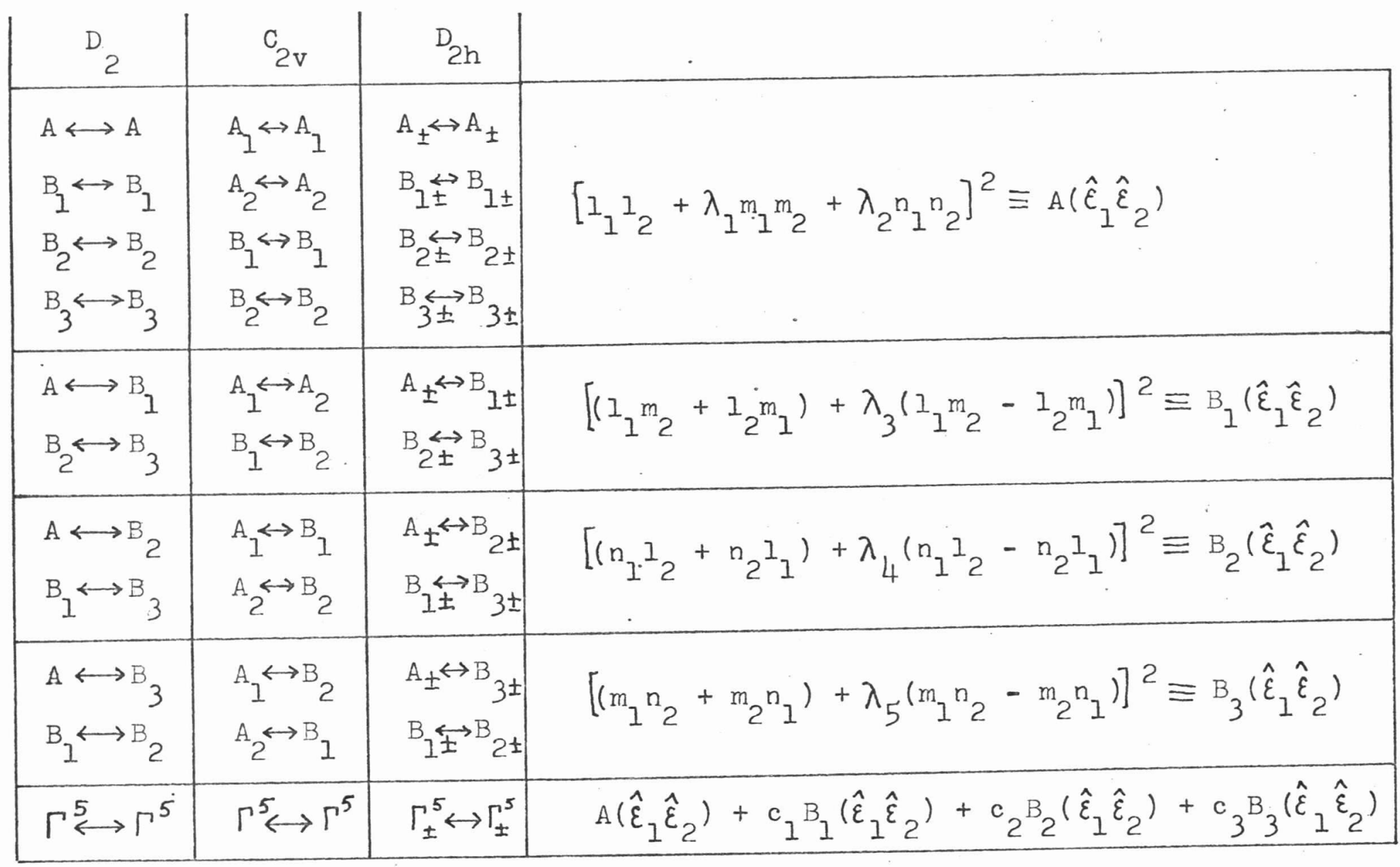




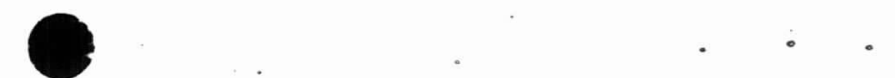

Table 3-1 (Contd.)

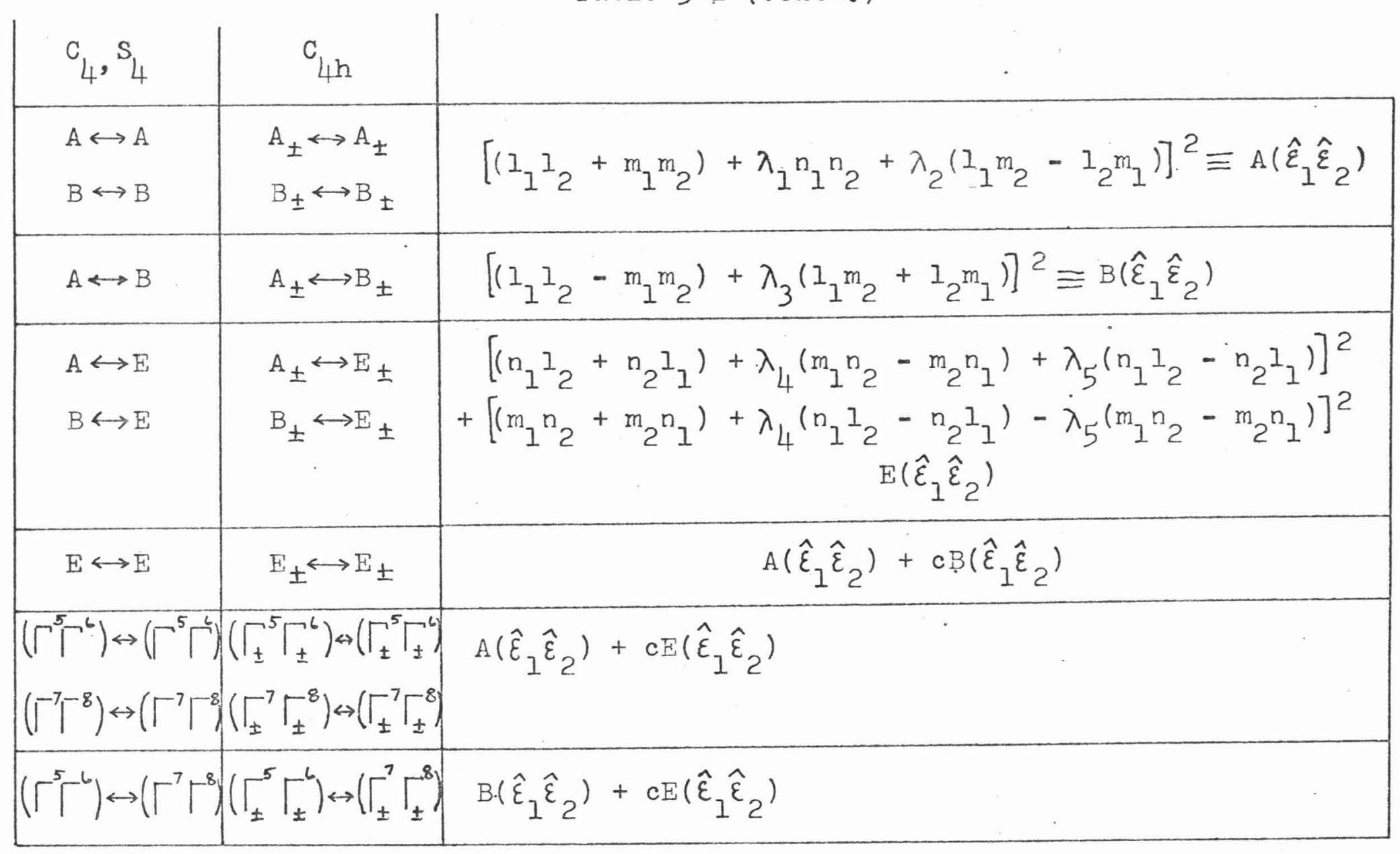

$\underset{\infty}{\infty}$ 
Table 3-1 (Contd.)

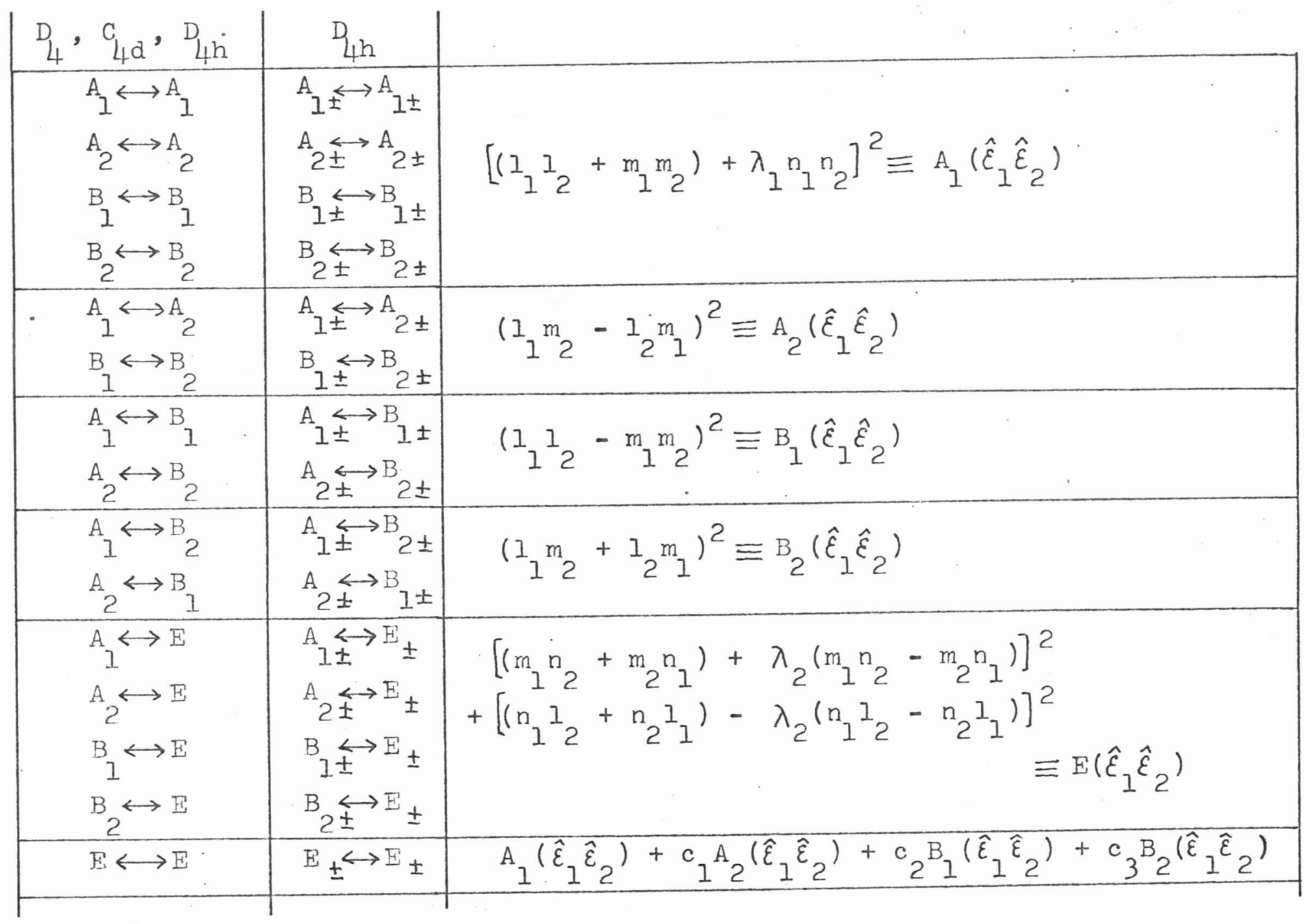

F 
Table 3-I (Contd.)

\begin{tabular}{|l|l|l|}
\hline$\Gamma^{6} \longleftrightarrow \Gamma^{6}$ & $\Gamma_{ \pm}^{6} \longleftrightarrow \Gamma_{ \pm}^{6}$ & $\mathrm{~A}_{1}\left(\hat{\varepsilon}_{1} \hat{\varepsilon}_{2}\right)+c_{1} \mathrm{~A}_{2}\left(\hat{\varepsilon}_{1} \hat{\varepsilon}_{2}\right)+c_{2} E\left(\hat{\varepsilon}_{1} \hat{\varepsilon}_{2}\right)$ \\
\hline$\Gamma^{7} \longleftrightarrow \Gamma^{7}$ & $\Gamma_{ \pm}^{7} \longleftrightarrow \Gamma_{ \pm}^{7}$ & \\
\hline$\Gamma^{6} \longleftrightarrow \Gamma^{7}$ & $\Gamma_{ \pm}^{6} \longleftrightarrow \Gamma_{ \pm}^{7}$ & $\mathrm{~B}_{1}\left(\hat{\varepsilon}_{I} \hat{\varepsilon}_{2}\right)+c_{I} B_{2}\left(\hat{\varepsilon}_{I} \hat{\varepsilon}_{2}\right)+c_{2} E\left(\hat{\varepsilon}_{1} \hat{\varepsilon}_{2}\right)$ \\
\hline
\end{tabular}

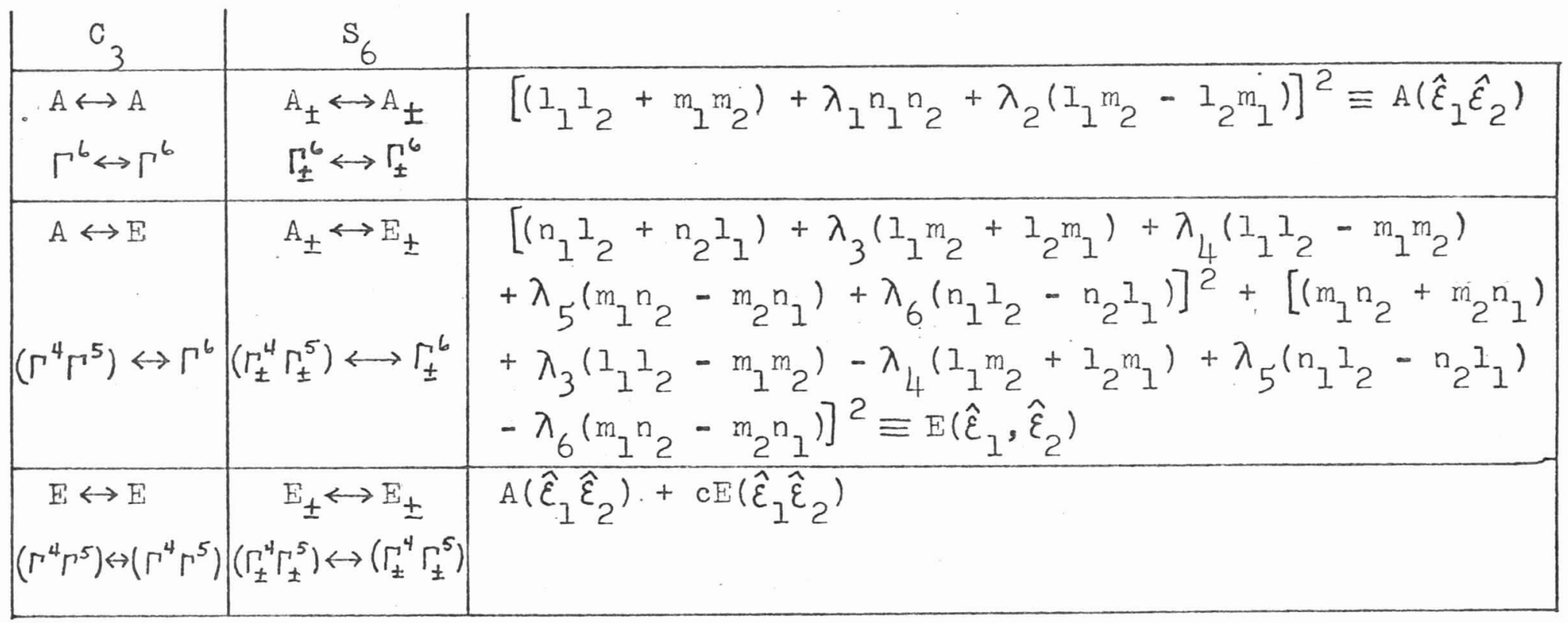

u 
Table 3-1 (Contd.)

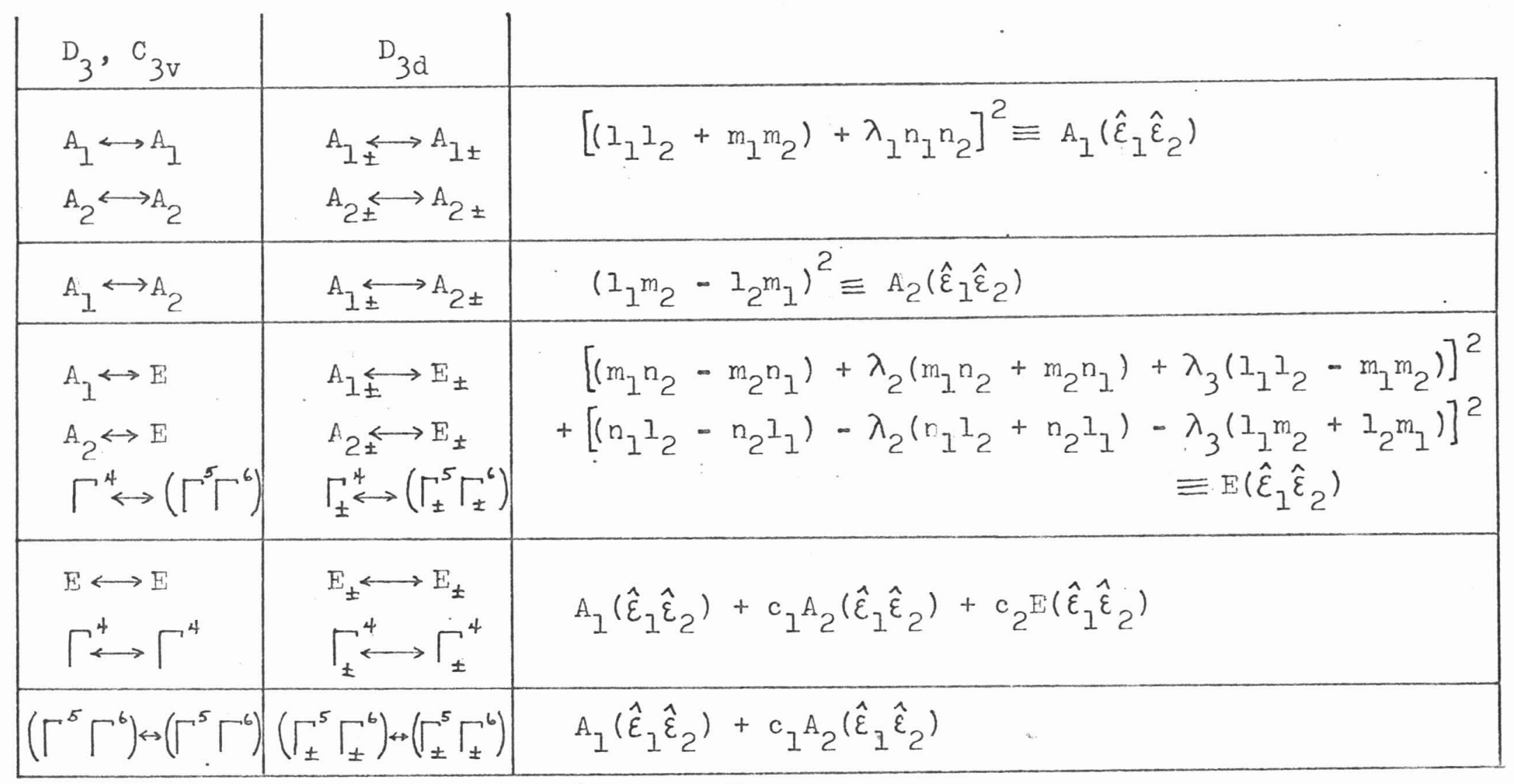


Table 3-1 (Contd.)

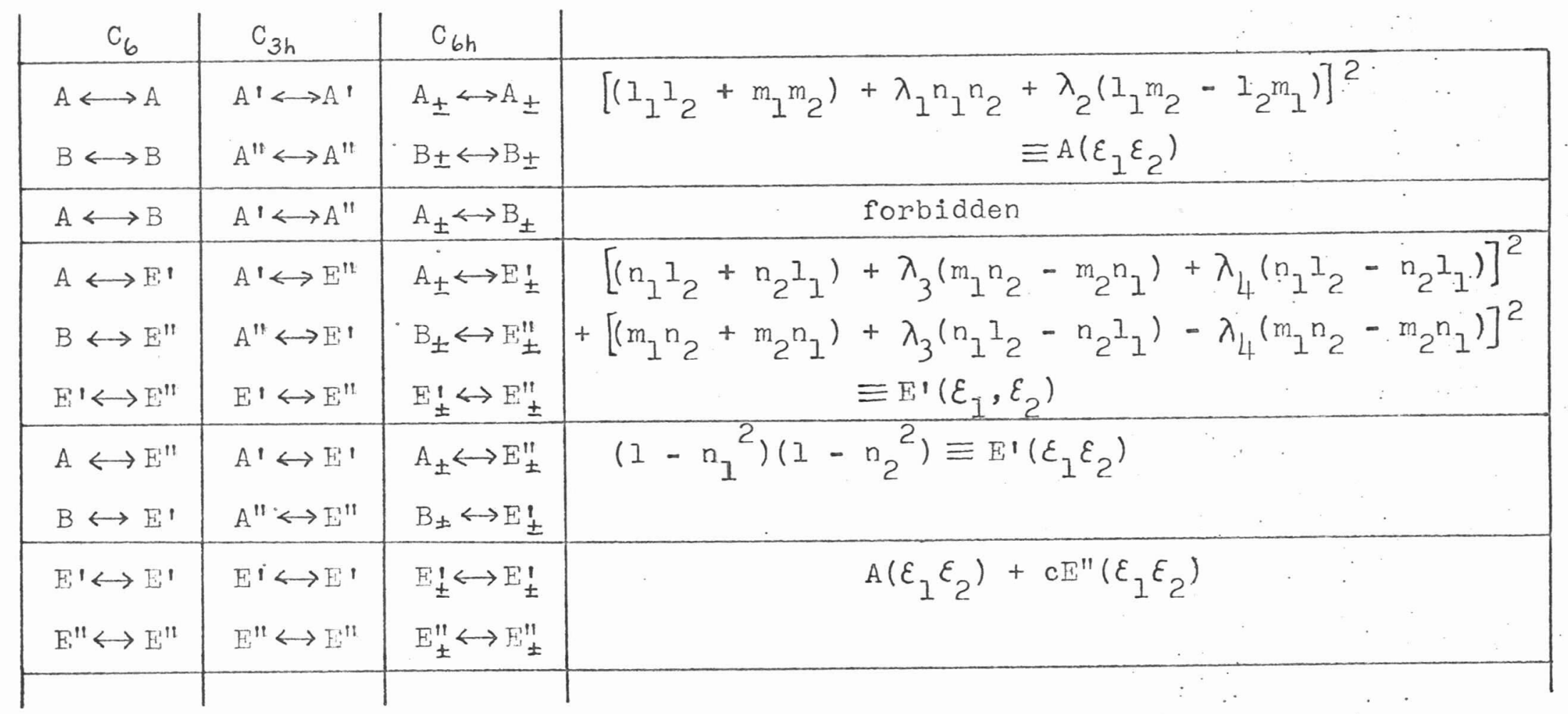

n 
Table 3-1 (Contd.)

\begin{tabular}{|c|c|}
\hline $\begin{array}{l}\left(\Gamma^{7} \Gamma^{8}\right) \leftrightarrow\left(\Gamma^{7} \Gamma^{8}\right)\left(\Gamma^{7} \Gamma^{8}\right) \leftrightarrow\left(\Gamma^{7} \Gamma^{8}\right)\left(\Gamma_{ \pm}^{7} \Gamma_{ \pm}^{8}\right) \leftrightarrow\left(\Gamma_{ \pm}^{7} \Gamma_{ \pm}^{8}\right) \\
\left(\Gamma^{9} \Gamma^{10}\right) \leftrightarrow\left(\Gamma^{9} \Gamma^{10}\right)\left(\Gamma^{9} \Gamma^{10}\right) \leftrightarrow\left(\Gamma^{9} \Gamma^{10}\right),\left(\Gamma_{ \pm}^{9} \Gamma_{ \pm}^{10}\right) \leftrightarrow\left(\Gamma_{ \pm}^{9} \Gamma_{ \pm}^{10}\right)\end{array}$ & $\mathrm{A}\left(\hat{\varepsilon}_{1}, \hat{\varepsilon}_{2}\right)+\mathrm{cE}\left(\hat{\varepsilon}_{1} \hat{\varepsilon}_{2}\right)$ \\
\hline$\left(\Gamma^{7} \Gamma^{8}\right) \leftrightarrow\left(\Gamma^{9} \Gamma^{10}\right)\left(\Gamma^{7} \Gamma^{8}\right) \leftrightarrow\left(\Gamma^{9} \Gamma^{10}\right)\left(\Gamma_{ \pm}^{7} \Gamma_{ \pm}^{8}\right) \leftrightarrow\left(\Gamma_{ \pm}^{9} \Gamma_{ \pm}^{10}\right)$ & $E^{\prime \prime}\left(\hat{\varepsilon}_{1} \hat{\varepsilon}_{2}\right)$ \\
\hline 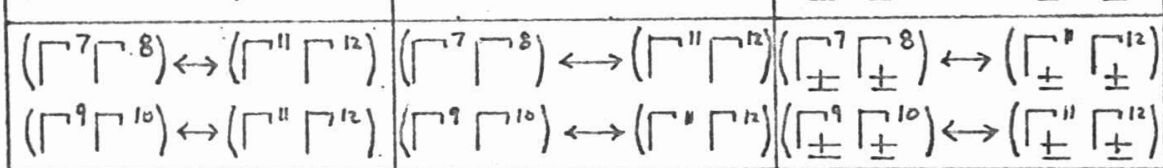 & $E^{\prime}\left(\hat{\varepsilon}_{1} \hat{\varepsilon}_{2}\right)+c E^{\prime \prime}\left(\hat{\varepsilon}_{1} \hat{\varepsilon}_{2}\right)$ \\
\hline$\left(\Gamma^{\prime \prime} \Gamma^{\prime 2}\right) \leftrightarrow\left(\Gamma^{\prime \prime} \Gamma^{\prime 2}\right)\left(\Gamma^{\prime \prime} \Gamma^{\prime 2}\right) \leftrightarrow\left(\Gamma^{\prime \prime} \Gamma^{\prime 2}\right)\left(\Gamma_{ \pm}^{\prime \prime} \Gamma_{ \pm}^{\prime 2}\right) \leftrightarrow\left(\Gamma_{ \pm}^{\prime \prime} \Gamma_{ \pm}^{\prime 2}\right)$ & $A\left(\hat{\varepsilon}_{1}, \hat{\varepsilon}_{2}\right)$ \\
\hline
\end{tabular}

\begin{tabular}{|c|c|c|c|}
\hline $\mathrm{D}_{6},{ }^{\mathrm{C}} 6 \mathrm{v}$ & $D_{3 h}$ & $D_{6 h}$ & \\
\hline $\begin{array}{l}\mathrm{A}_{1} \leftrightarrow \mathrm{A}_{1} \\
\mathrm{~A}_{2} \leftrightarrow \mathrm{A}_{2} \\
\mathrm{~B}_{1} \leftrightarrow \mathrm{B}_{1} \\
\mathrm{~B}_{2} \leftrightarrow \mathrm{B}_{2}\end{array}$ & $\begin{array}{l}A_{1}^{\prime} \leftrightarrow A_{1}^{\prime} \\
A_{2}^{\prime} \leftrightarrow A_{2}^{\prime} \\
A_{1}^{\prime \prime} \leftrightarrow A_{1}^{\prime \prime} \\
A_{2}^{\prime \prime} \leftrightarrow A_{2}^{\prime \prime}\end{array}$ & $\begin{array}{l}\mathrm{A}_{1 \pm} \leftrightarrow \mathrm{A}_{1 \pm} \\
\mathrm{A}_{2} \leftrightarrow \mathrm{A}_{2 \pm} \\
\mathrm{B}_{1 \pm} \leftrightarrow \mathrm{B}_{1 \pm} \\
\mathrm{B}_{2} \leftrightarrow \mathrm{B}_{2 \pm}\end{array}$ & {$\left[\left(I_{1} I_{2}+m_{1} m_{2}\right)+\lambda_{1} n_{1} n_{2}\right]^{2} \equiv A_{1}\left(\hat{\varepsilon}_{1} \hat{\varepsilon}_{2}\right)$} \\
\hline $\begin{array}{l}\mathrm{A}_{1} \leftrightarrow \mathrm{A}_{2} \\
\mathrm{~B}_{1} \leftrightarrow \mathrm{B}_{\mathrm{C}}\end{array}$ & $\begin{array}{l}A_{1}^{\prime} \leftrightarrow A_{2}^{\prime} \\
A_{1}^{\prime \prime} \leftrightarrow A_{2}^{\prime \prime}\end{array}$ & $\begin{array}{l}\mathrm{A}_{1 \pm} \leftrightarrow \mathrm{A}_{2 \pm} \\
\mathrm{B}_{1 \pm} \leftrightarrow \mathrm{B}_{2 \pm}\end{array}$ & $\left(I_{1} m_{2}-I_{2} m_{1}\right)^{2} \equiv A_{2}\left(\hat{\varepsilon}_{1} \hat{\varepsilon}_{2}\right)$ \\
\hline
\end{tabular}


Table 3-I"(Contd.)

\begin{tabular}{|c|c|c|c|}
\hline $\begin{array}{l}A_{1} \leftrightarrow B_{1} \\
A_{1} \leftrightarrow B_{2} \\
A_{2} \leftrightarrow B_{1} \\
A_{2} \leftrightarrow B_{2}\end{array}$ & $\begin{aligned} A_{1}^{\prime} \leftrightarrow A_{1}^{\prime \prime} \\
A_{1}^{\prime} \leftrightarrow A_{2}^{\prime \prime} \\
A_{2}^{\prime} \leftrightarrow A_{1}^{\prime \prime} \\
A_{2}^{\prime} \leftrightarrow A_{2}^{\prime \prime}\end{aligned}$ & 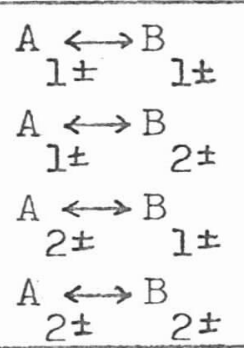 & forbidden \\
\hline $\begin{array}{l}\mathrm{A}_{2} \leftrightarrow \mathrm{E}_{1} \\
\mathrm{~A}_{2} \leftrightarrow \mathrm{E}_{1} \\
\mathrm{~B}_{1} \leftrightarrow \mathrm{E}_{2} \\
\mathrm{~B}_{2} \leftrightarrow \mathrm{E}_{2} \\
\mathrm{E}_{1} \leftrightarrow \mathrm{E}_{2}\end{array}$ & $\begin{array}{l}A_{1}^{\prime} \leftrightarrow E^{\prime \prime} \\
A_{2}^{\prime} \leftrightarrow E^{\prime \prime} \\
2 \\
A_{2}^{\prime \prime} \leftrightarrow E^{\prime} \\
A_{1}^{\prime \prime} \leftrightarrow E^{\prime} \\
E^{\prime} \leftrightarrow E^{\prime \prime}\end{array}$ & 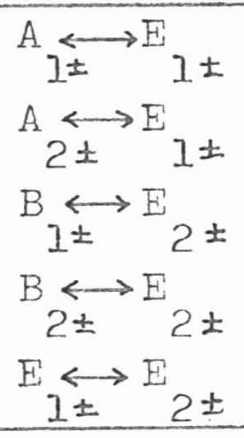 & $\begin{aligned} & {\left[\left(m_{1} n_{2}-m_{2} n_{1}\right)+\lambda_{2}\left(m_{1} n_{2}+m_{2} n_{1}\right)\right]^{2} } \\
+ & {\left[\left(n_{1} I_{2}-n_{2} I_{1}\right)-\lambda_{2}\left(n_{1} I_{2}+n_{2} I_{1}\right)\right]^{2} } \\
& \equiv E_{1}\left(\hat{\varepsilon}_{1} \hat{\varepsilon}_{2}\right) .\end{aligned}$ \\
\hline $\begin{array}{l}A_{1} \leftrightarrow E_{2} \\
A_{2} \leftrightarrow E_{2} \\
B_{1} \leftrightarrow E_{1} \\
B_{2} \leftrightarrow E_{1}\end{array}$ & $\begin{array}{l}A_{1}^{\prime} \leftrightarrow E^{\prime} \\
A_{2}^{\prime} \leftrightarrow E^{\prime} \\
2 \\
A^{\prime \prime} \leftrightarrow E^{\prime \prime} \\
2 \\
A_{1}^{\prime \prime} \leftrightarrow E^{\prime \prime}\end{array}$ & 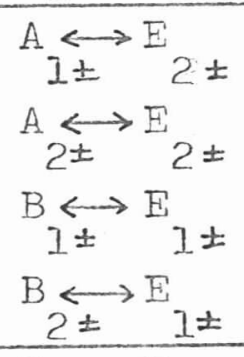 & $\left(1-n_{1}^{2}\right)\left(1-n_{2}^{2}\right) \equiv E_{2}\left(\hat{\varepsilon}_{1} \hat{\varepsilon}_{2}\right)$ \\
\hline $\begin{array}{l}\mathrm{E}_{1} \leftrightarrow \mathrm{E}_{1} \\
\mathrm{E}_{2} \leftrightarrow \mathrm{E}_{2}\end{array}$ & $\begin{array}{l}E^{\prime \prime} \longleftrightarrow E^{\prime \prime} \\
E^{\prime} \longleftrightarrow E^{\prime}\end{array}$ & $\begin{array}{l}E_{I \pm} E_{1 \pm} \\
E_{2 \pm}^{\longleftrightarrow} E_{2 \pm}\end{array}$ & $A_{1}\left(\hat{\varepsilon}_{1} \hat{\varepsilon}_{2}\right)+c_{1} A_{2}\left(\hat{\varepsilon}_{1} \hat{\varepsilon}_{2}\right)+c_{2} E_{2}\left(\hat{\varepsilon}_{1} \hat{\varepsilon}_{2}\right)$ \\
\hline
\end{tabular}


Table 3-1 (Contd.)

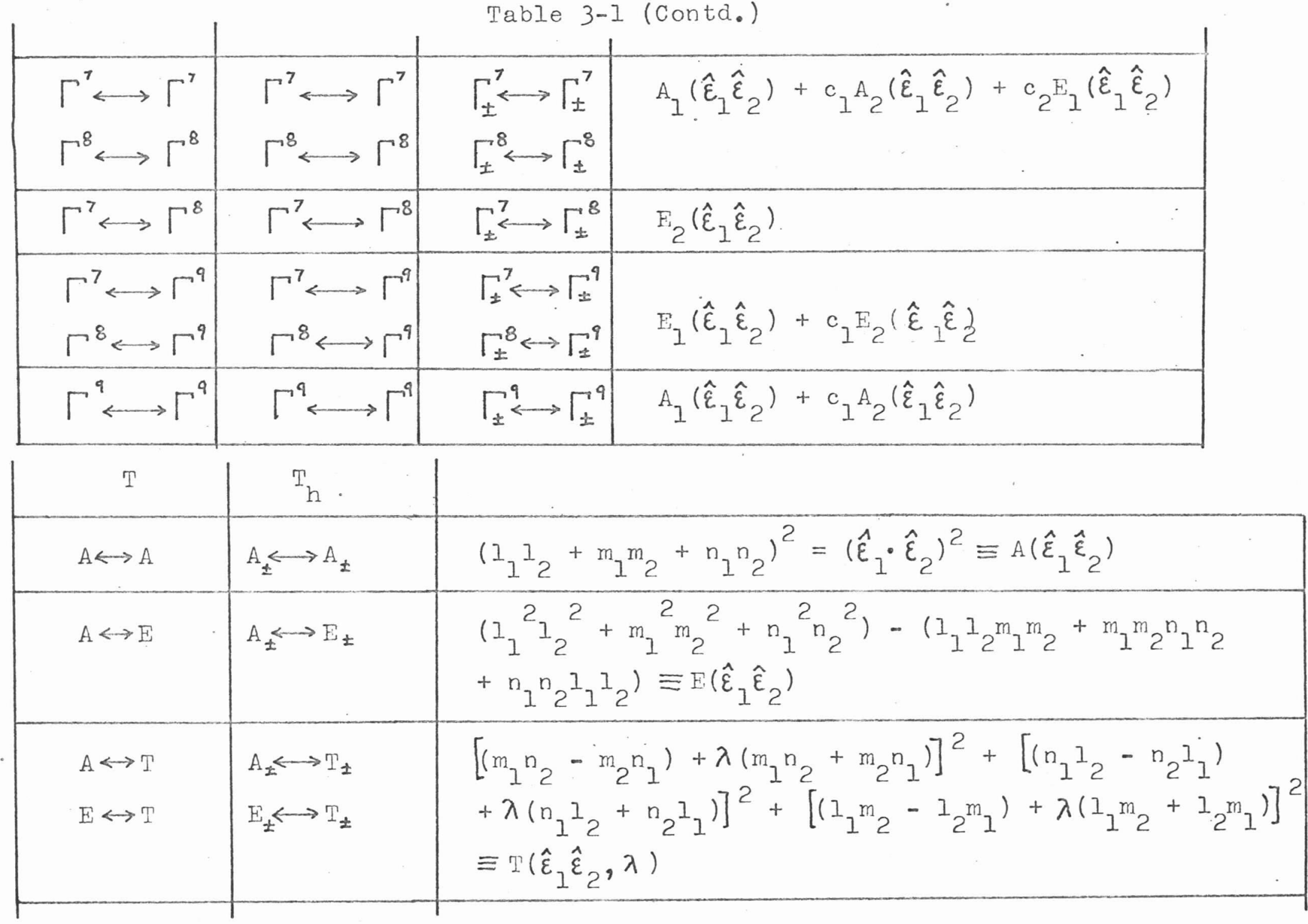

v 
Table 3-1 (Contd.)

\begin{tabular}{|c|c|l|} 
& & \\
\hline$E \leftrightarrow E$ & $E_{ \pm} \longleftrightarrow E_{ \pm}$ & $A\left(\hat{\varepsilon}_{I} \hat{\varepsilon}_{2}\right)+c E\left(\hat{\varepsilon}_{I} \hat{\varepsilon}_{2}\right)$ \\
\hline$T \leftrightarrow T$ & $T_{ \pm} \longleftrightarrow T_{ \pm}$ & $A\left(\hat{\varepsilon}_{I} \hat{\varepsilon}_{2}\right)+c_{I} E\left(\hat{\varepsilon}_{I} \hat{\varepsilon}_{2}\right)+c_{2} T\left(\hat{\varepsilon}_{1} \hat{\varepsilon}_{2}, \lambda\right)+c_{3} T\left(\hat{\varepsilon}_{1} \hat{\varepsilon}_{2}, \lambda^{\prime}\right)$ \\
\hline$\Gamma^{5} \leftrightarrow \Gamma^{5}$ & $\Gamma_{ \pm}^{5} \longleftrightarrow \Gamma_{ \pm}^{5}$ & $A\left(\hat{\varepsilon}_{I} \hat{\varepsilon}_{2}\right)+c_{I}\left(\hat{\varepsilon}_{1} \hat{\varepsilon}_{2,}, \lambda\right)$ \\
\hline$\Gamma^{5} \leftrightarrow\left(\Gamma^{6} \Gamma^{7}\right)$ & $\Gamma_{ \pm}^{5} \leftrightarrow\left(\Gamma_{ \pm}^{6} \Gamma_{ \pm}^{7}\right)$ & $E\left(\hat{\varepsilon}_{I} \hat{\varepsilon}_{2}\right)+c T\left(\hat{\varepsilon}_{1} \hat{\varepsilon}_{2,} \lambda\right)$ \\
\hline$\left(\Gamma^{6} \Gamma^{7}\right) \leftrightarrow\left(\Gamma^{6} \Gamma^{7}\right)$ & $\left(\Gamma_{ \pm}^{6} \Gamma_{ \pm}^{7}\right) \Leftrightarrow\left(\Gamma_{ \pm}^{6} \Gamma_{ \pm}^{7}\right)$ & $A\left(\hat{\varepsilon}_{I} \hat{\varepsilon}_{2}\right)+c_{I} E\left(\hat{\varepsilon}_{I} \hat{\varepsilon}_{2}\right)+c_{2} T\left(\hat{\varepsilon}_{1} \hat{\varepsilon}_{2}, \lambda\right)$ \\
\hline
\end{tabular}

\begin{tabular}{|c|c|c|}
\hline $0, \mathrm{~T}_{\mathrm{d}}$ & $\mathrm{O}_{\mathrm{h}}$ & \\
\hline $\begin{array}{l}A_{1} \longleftrightarrow A_{1} \\
A_{2} \longleftrightarrow A_{2}\end{array}$ & $\begin{array}{l}A_{1} \longleftrightarrow A_{1 \pm} \\
A_{2 \pm} \longleftrightarrow A_{2 \pm}\end{array}$ & $\left(I_{1} I_{2}+m_{1} m_{2}+n_{1} n_{2}\right)^{2} \equiv A_{1}\left(\hat{\varepsilon}_{1} \hat{\varepsilon}_{2}\right)$ \\
\hline$A_{1} \longleftrightarrow A_{2}$. & $\mathrm{A}_{1} \leftrightarrows \mathrm{A}_{2 \pm}$ & forbidden \\
\hline $\begin{array}{l}A_{1} \longleftrightarrow E \\
A_{2} \longleftrightarrow E\end{array}$ & $\begin{array}{l}\mathrm{A}_{1 \pm} \longrightarrow \mathrm{E}_{ \pm} \\
\mathrm{A}_{2 \pm} \longleftrightarrow \mathrm{E}_{ \pm}\end{array}$ & $\begin{aligned}\left(I_{1}^{2} I_{2}^{2}+\right. & \left.m_{1}^{2} m_{2}^{2}+n_{1}^{2} n_{2}^{2}\right)-\left(I_{1} I_{2} m_{1} m_{2}+m_{1} m_{2} n_{1} n_{2}{ }^{+}\right. \\
& \left.n_{1} n_{2} I_{1} I_{2}\right) \equiv E\left(\hat{\varepsilon}_{1} \hat{\varepsilon}_{2}\right)\end{aligned}$ \\
\hline $\begin{array}{l}A_{1} \longleftrightarrow T_{1} \\
A_{2} \longleftrightarrow T_{2}\end{array}$ & $\begin{array}{l}\mathrm{A}_{I_{ \pm}} \longleftrightarrow \mathrm{T}_{I_{ \pm}} \\
\mathrm{A}_{2 \pm} \longleftrightarrow \mathbb{T}_{2 \pm}\end{array}$ & $\begin{aligned}\left(m_{1} n_{2}\right. & \left.-m_{2} n_{1}\right)^{2}+\left(I_{1} n_{2}-I_{2} n_{1}\right)^{2} \\
& +\left(I_{1} m_{2}-I_{2} m_{1}\right)^{2}=\left(\hat{\varepsilon}_{1} \times \hat{\varepsilon}_{2}\right)^{2} \equiv T_{1}\left(\hat{\varepsilon}_{1} \hat{\varepsilon}_{2}\right)\end{aligned}$ \\
\hline
\end{tabular}


Table 3-1 (Contd.)

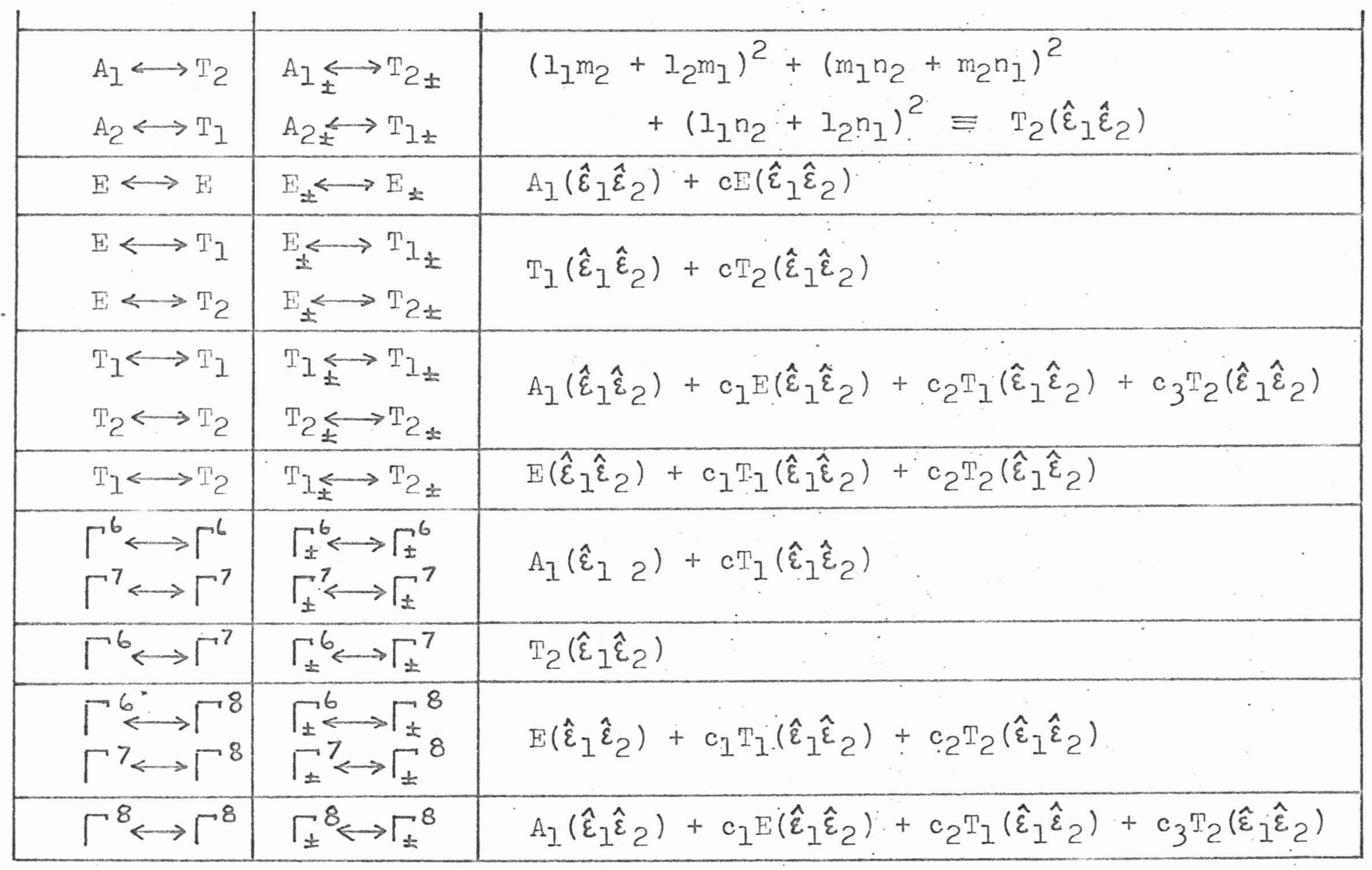

g 

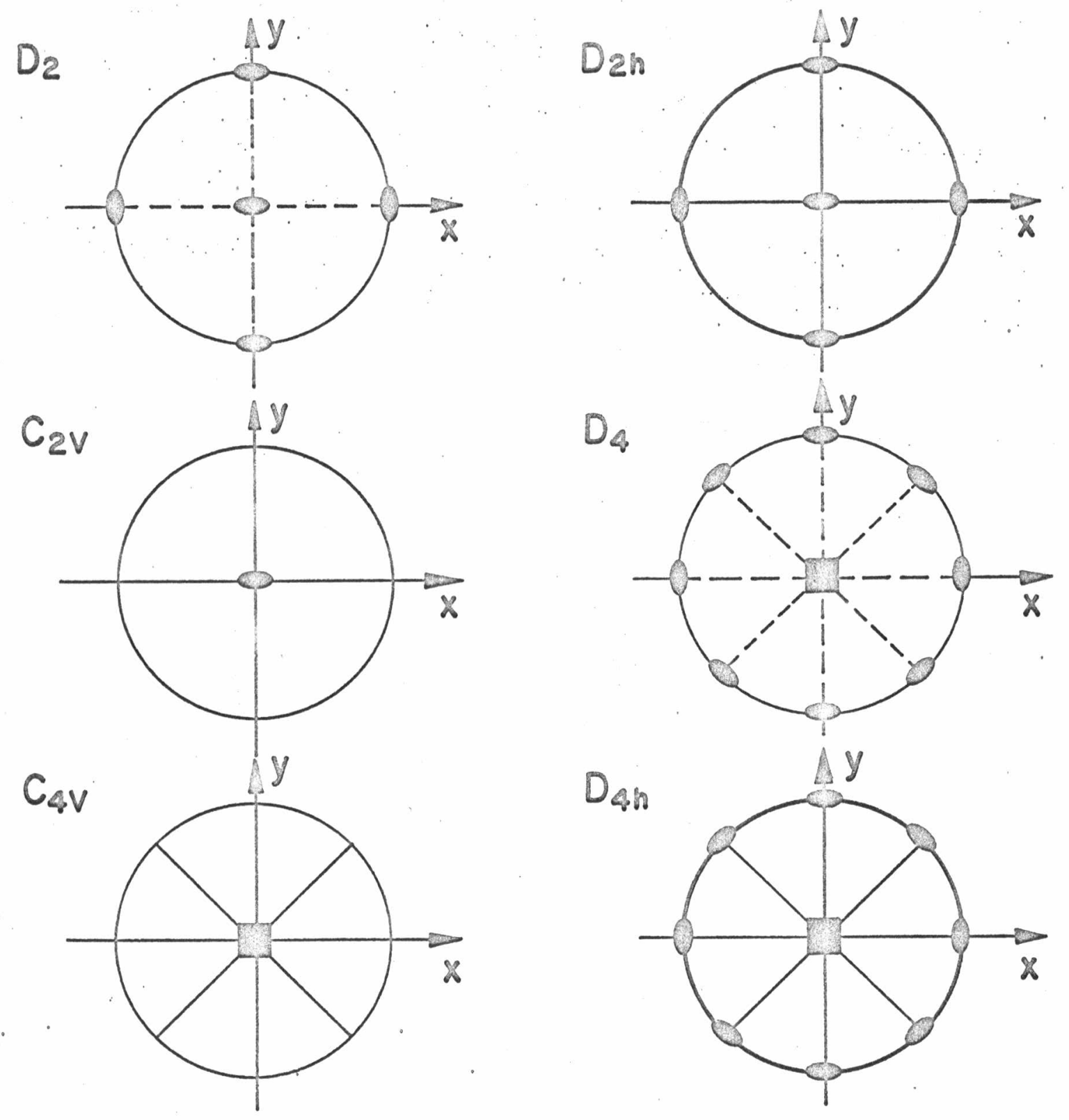

Fig.3-1. Definition of axes 

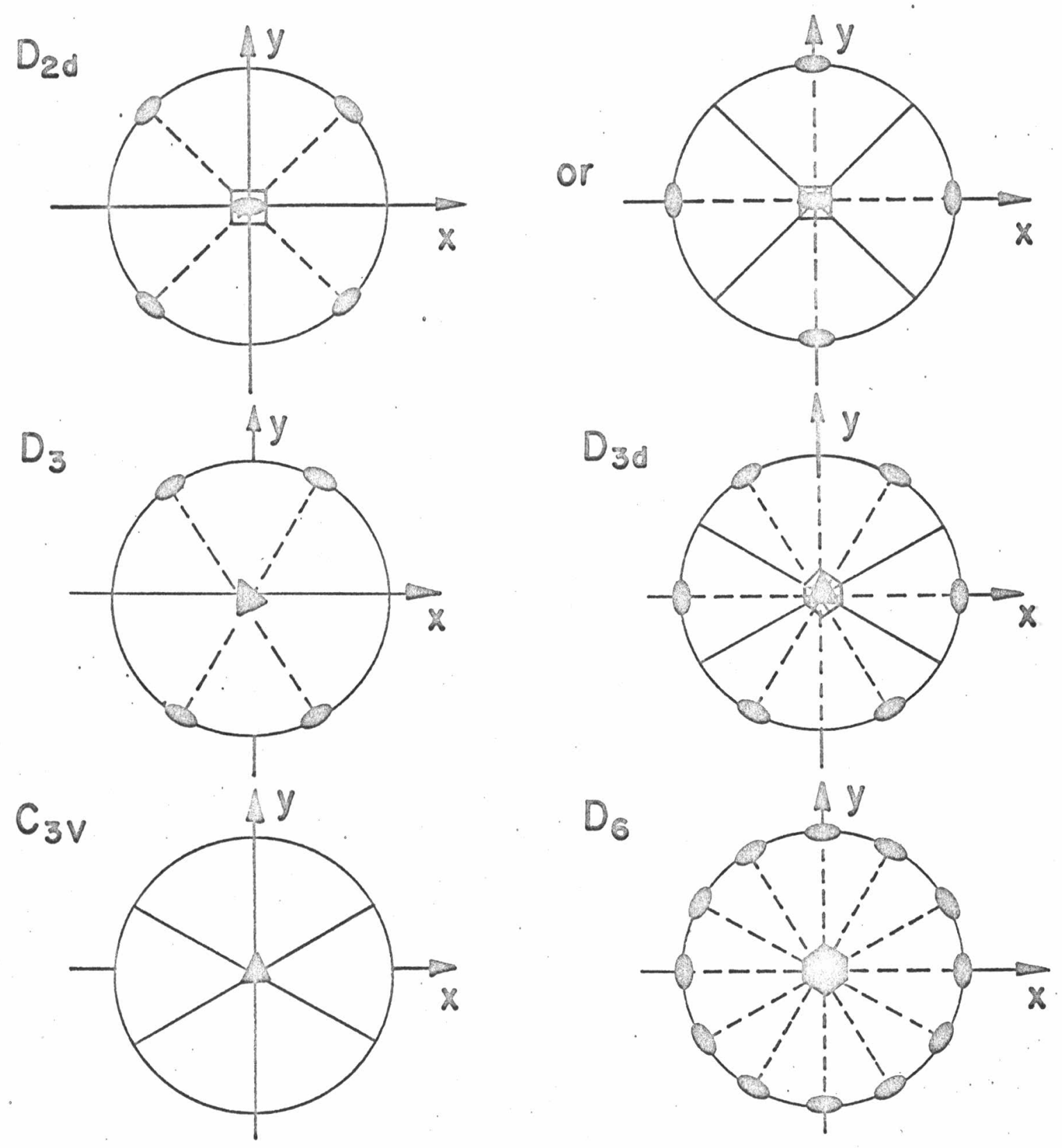

Fig.3-2. Definition of axes 
$c_{6 V}$

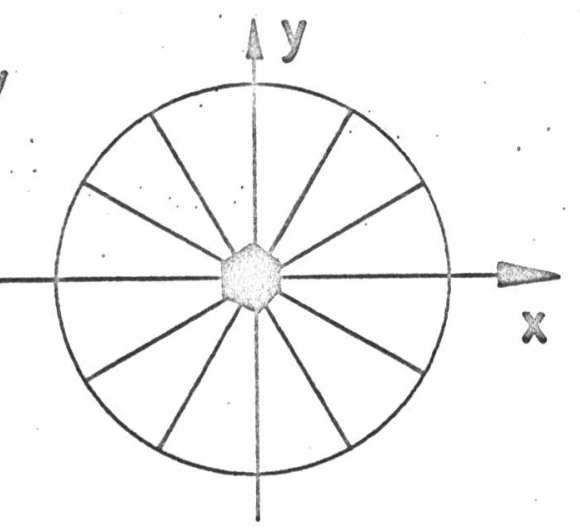

$D_{3 h}$

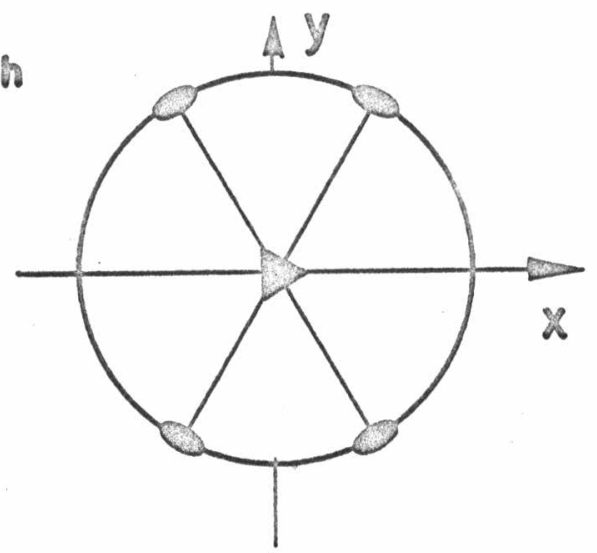

$D_{\text {en }}$

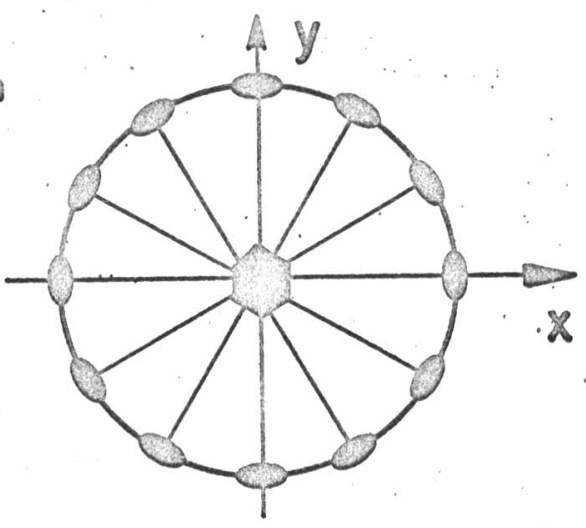

Or

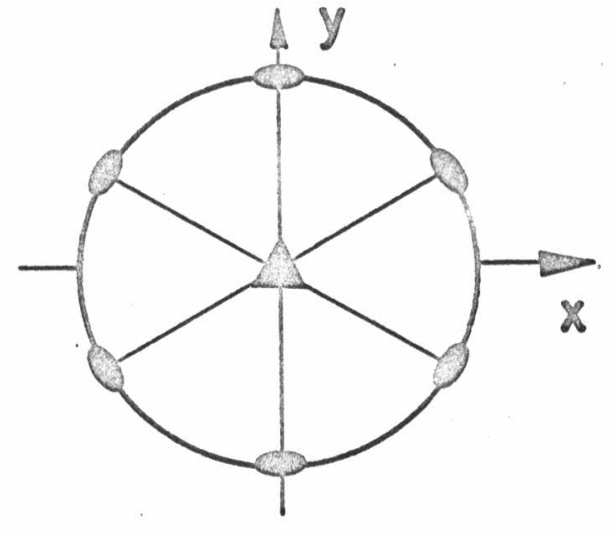

Fig.3-3. Definition of axes 
$T, T_{d}$, and $T_{h}$
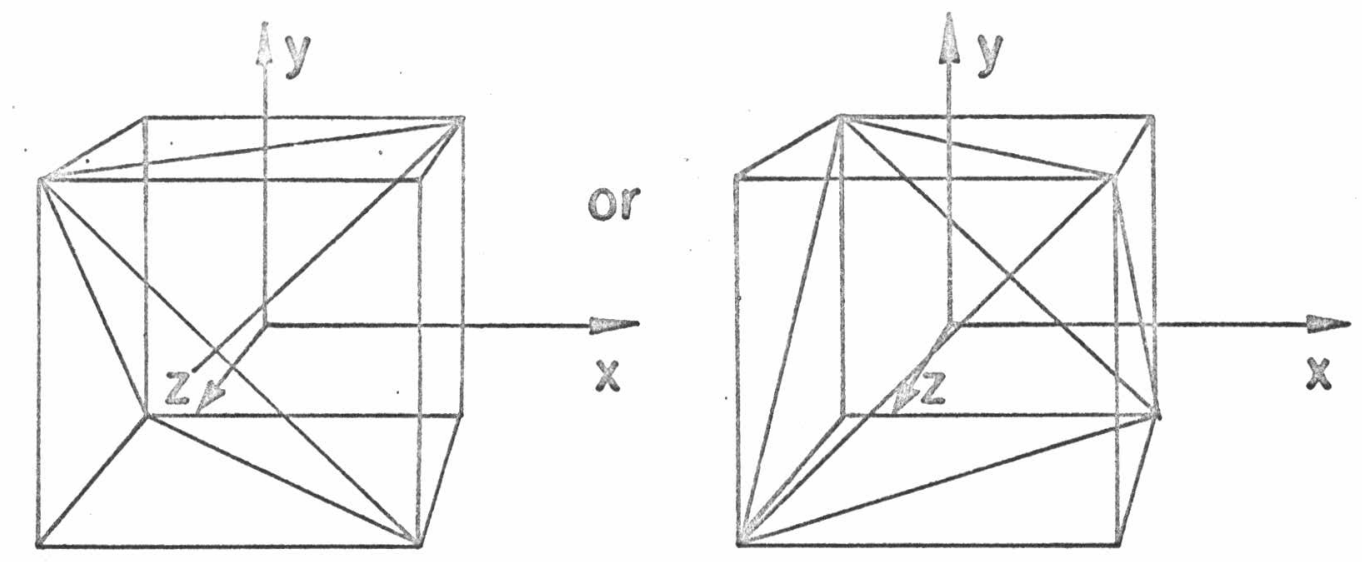

$O$ and $O_{h}$

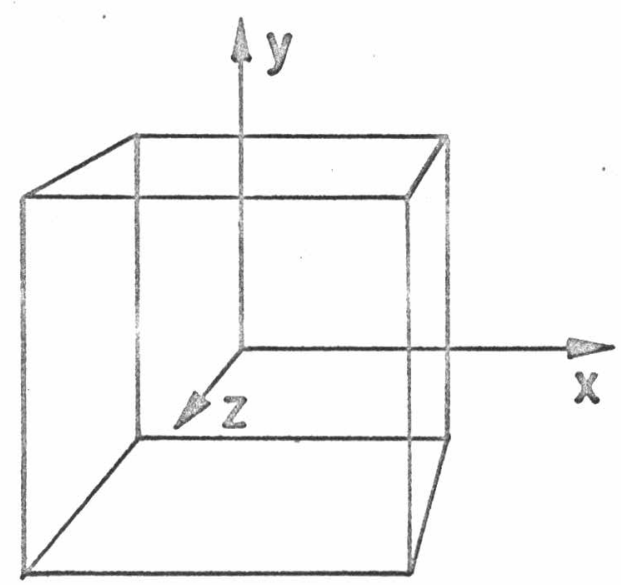

Fig.3-4. Definition of axes 
C. Transitions at Non-central Symmetry Points

It often happens that energy band minima occur at points in the Brillouin zone otwer than the $\Gamma$-point, and these minima are most frequently found at points having higher symmetry than that of a general point in the zone. 40 It is therefore useful to determine the angular dependence of two-photon absorption for transitions taking place at these symmetry points.

Perhaps the most airect approach to the problem is to note that $\left|c_{i}\left(k_{0}\right)\right\rangle$ and $\left|v_{j}\left(k_{0}\right)\right\rangle$ transform according to irreducible representations, say $D^{\lambda k_{0}}$ and $D^{\nu k_{0}}$, of the space group of the crystal. 43 men we could apply Eq.3-3 directiy and decompose $D^{\lambda k_{0}} \times D^{\nu k_{0}}$ into irreducible representations of the space group. Since $\hat{\mathcal{E}}_{1} \cdot \overrightarrow{\mathrm{T}} \cdot \hat{\varepsilon}_{2}$ transforms entirely within the set of space group representations for which $\vec{k}=0$, we would require only the knowledge of which point group representations are contained in $D^{\lambda k_{0}} \times D^{\nu k_{0}}$. Unfortunately the Kronecker multiplication properties of space group representations are not accessible. There is an alternative procedure, however, which requires only the knowledge of the representations of the group of the wave vector.

When an element of the space group operates on $\left|v_{j}\left(k_{0}\right)\right\rangle$, it either produces a state having wave vector $\vec{k}_{0}$ or it generates a state with wave vector in the star 40 of $\vec{k}_{0}$. Let us choose from the space group a set of $\mathbb{N}$ 
elements $\{P(R)\}$ which generates the $\mathbb{N}$-pointed star of. $\vec{k}_{0}$. Thus we can write 43

$$
P(R)\left|V_{j}\left(k_{0}\right)\right\rangle=\left|V_{j}\left(R k_{0}\right)\right\rangle
$$

and hence

$$
\begin{aligned}
& \sum_{k_{0}^{\prime} i j}\left|\left\langle c_{i}\left(k_{0}^{\prime}\right)\left|\hat{\varepsilon}_{1} \cdot \vec{T} \cdot \hat{\varepsilon}_{2}\right| v_{j}\left(k_{0}^{\prime}\right)\right\rangle\right|^{2} \\
& =\sum_{R_{i j}}\left|\left\langle c_{i}\left(R k_{0}\right)\left|\hat{\varepsilon}_{i} \cdot \overleftrightarrow{T} \cdot \hat{\varepsilon}_{2}\right| v_{j}\left(R k_{0}\right)\right\rangle\right|^{2}
\end{aligned}
$$

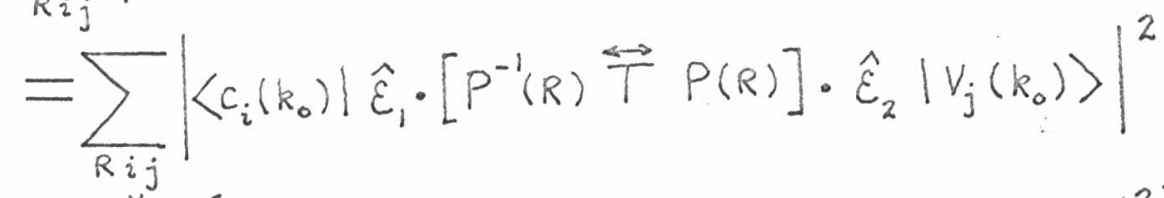

$$
\begin{aligned}
& =\sum_{R=1}^{N}\left\{\sum\left|\left\langle c_{i}\left(k_{0}\right)\left|\left[P(R) \hat{\varepsilon}_{1}\right] \cdot \overleftrightarrow{T} \cdot\left[P(R) \hat{\varepsilon}_{2}\right]\right| v_{j}\left(k_{0}\right)\right\rangle\right|^{2}\right\}
\end{aligned}
$$

The procedure, then, is to find the angular function for a transition at $\vec{k}_{0}$ between states belonging to arbitrary irreducible representations of the group of $\vec{k}_{0}$ and then to add for each $P(R)$ a similar function with $\hat{\varepsilon}_{\text {, }}$ and $\hat{\varepsilon}_{1}$ replaced by $P(R) \hat{\varepsilon}_{1}$ and $P(R) \hat{\varepsilon}_{2}$.

The angular dependence functions for transitions of the type $d^{\prime} \rightarrow d^{\lambda}$, where $d^{\lambda}$ is a representation of the group of the wave vector, at non-central symmetry points for simple and face-centered cubic crystals and for zinc blende and diamond structures, have been calculated by Inoue and royozawa. ${ }^{11}$ we can obtain the angular dependence for more general $d^{\nu} \rightarrow a^{\lambda}$ transitions from Inoue and 
Toyozawa's functions with the use of rable 3-1. For example, the group of $\Delta$ for a face-centered cubic crystal is $C_{4 V^{*}}$. The angular function for $\Delta^{\nu} \longrightarrow \Delta^{\lambda}$ is that given by $\Gamma^{\nu} \longrightarrow \Gamma^{\lambda}$ of $\mathrm{C}_{4 V}$ in Table $3-I$ with the $\gamma^{\mu}\left(\hat{\varepsilon}_{1} \hat{\varepsilon}_{2}\right)$ replaced by the $\Delta^{\mu}\left(\hat{\varepsilon}_{1} \hat{\varepsilon}_{2}\right)$ in the table of Inoue and Toyozawa. For reference their table is reproduced in Appendix $\mathbb{E}$, which also contains the Brillouin zones and definitions of the labels of symmetry lines and points for zinc blende, diamond, face-centered cubic, body-centered cubic and simple cubic structures ( $F i g . E-I$ )。

The angular functions for two more space groups have been calculated and are given below. These are for the hexagonal close-packed. structure (space group $D_{6 h}^{4}$ ) and the wurtzite structure $\left(\mathrm{C}_{6 \mathrm{~V}}^{4}\right)$. Wurtzite differs from HCP in that it does not contain a replection plane perpendicular to the c axis. Both groups contain point operations associated with fractional lattice translations. Much of the general theory of space group representations has been given by Seitz. 44 Extensive use of his results was made by Bouckaert, Smoluchowski and $W^{2}$ gner ${ }^{40}$ for some simple crystal structures. Herring 45 extended the method of space group reduction to more complicated groups, and this was extended further by Elifott ${ }^{46}$ to include the effects of spin-orbit coupling. The character tables for. the hexagonal close-packed structure were derived by Herring 45 and Elliott ${ }^{46}$ and we use their notation. For wurtzite we 
use the notation of Glasser 47 and of casella. 48 The Brillouin zone and definition of symmetry lines and points - are given in Fig.3-5.

A comment should be made about the calculation of the angular functions. The group of a wave vector other than $\vec{k}=0$ is usualiy rather complicated, and it is rarely isomorphic (or homomorphic) to one of the point groups. It is necessary to detemine which representations of the group of $\vec{k}$ have basis functions contained in the operator $\vec{T}$; that is, to which representations do the components of $\vec{T}$ belong. This is easily accomplished if one notes that frequently the entire character table is not given for the group of $\vec{k}$. Consider, for example, the character table for the group of A in HCP. The single-valued representations $\mathrm{A}_{1}, \mathrm{~A}_{2}$ and $\mathrm{A}_{3}$ reported by Herring have dimensionalities 2, 2 and 4 , respectively, as do the double-valued representations $A_{4}, A_{5}$ and $A_{6}$ calculated by Elliott. These, obviously, cannot constitute the entire character table for the double group of $A$, because they do not contain the identity representation; they are only the representations to which a Bloch function of wave vector $\vec{k}_{A}$ can belong. The entire group of $A$ is given in Table 3-2. The representations $a_{\mu}$ are those to which the tensor components belong. Just how the tensor components are related to the representations becomes apparent when it is noticed that the representations $a_{\mu}$ are those of the point group 
Table 3-2: Complete character table for the double group of A.

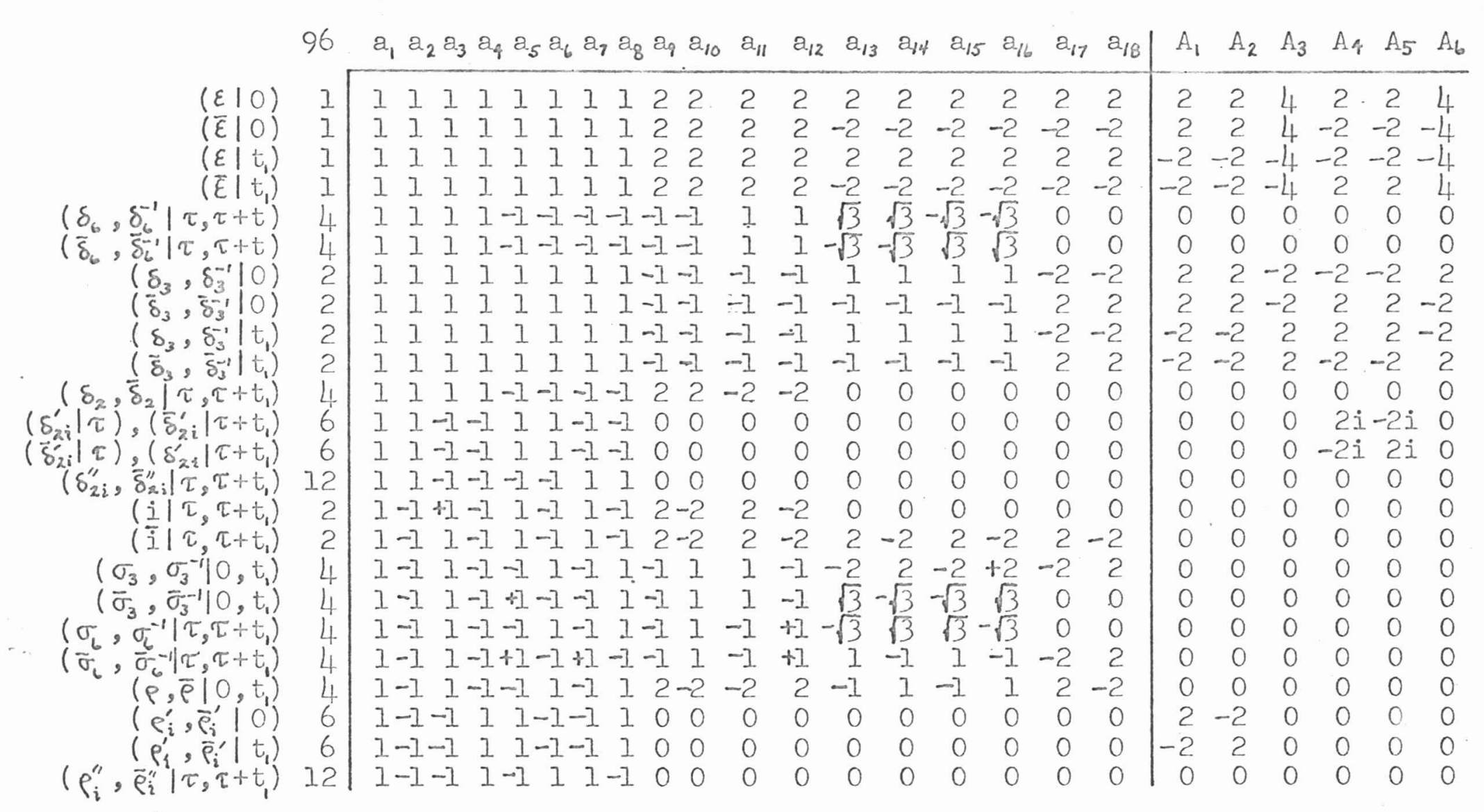


$\mathrm{C}_{6 \mathrm{v}}$, the properties of which are well known. In effect, then, what we must do to calculate the angular functions

- for $A^{\nu} \longrightarrow A^{\lambda}$ is to decompose $A^{\nu} \times A^{\lambda}$ into irreducible representations $a_{\mu}$. The angular dependence is given by $\sum_{\mu} a_{\mu}\left(\hat{\varepsilon}_{1} \hat{\varepsilon}_{2}\right)$ where the $a_{\mu}\left(\hat{\varepsilon}_{1} \hat{\varepsilon}_{2}\right)$ are the functions defined in rable $3-1$ for " $6 v$, and $\mu$ runs over the representations contained in $\mathrm{A}^{\nu} \times \mathrm{A}^{\lambda}$. For a point of lower symmetry than $A$, that is, a point having more than one point in its star, we must sum over $R$ as in Eq.3-7. The angular functions for all possible transitions at the symmetry points $\Gamma, K, A, H, M$ and $I$ and on lines $\Delta$, $\Sigma, U, P, T$ and $S$ are given for HCP in Table $3-3$ and for wurtzite in Table 3-4. 
Table 3-3

Angular dependence functions for hexagonal close-packed structure. Notation used is that of Herring (Ref. 45) for the singlewvalued representations and Elliott (Ref. 46) for the double-valued representations.

\begin{tabular}{|c|c|c|c|}
\hline$\Gamma$ & $\mathrm{K}$ & $\Delta$ & \\
\hline 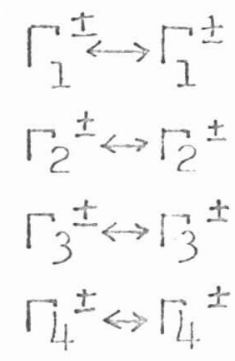 & $\begin{array}{l}\mathrm{K}_{1} \leftrightarrow \mathrm{K}_{1} \\
\mathrm{~K}_{2} \leftrightarrow \mathrm{K}_{2} \\
\mathrm{~K}_{3} \leftrightarrow \mathrm{K}_{3} \\
\mathrm{~K}_{4} \leftrightarrow \mathrm{K}_{4}\end{array}$ & $\begin{array}{l}\Delta_{1} \leftrightarrow \Delta_{1} \\
\Delta_{2} \leftrightarrow \Delta_{2} \\
\Delta_{3} \leftrightarrow \Delta_{3} \\
\Delta_{4} \leftrightarrow \Delta_{4}\end{array}$ & $\begin{aligned} {\left[\left(I_{1} I_{2}+m_{1} m_{2}\right)\right.} & \left.+\lambda_{1} n_{1} n_{2}\right]^{2} \\
& \equiv A_{1}\left(\hat{\varepsilon}_{1} \hat{\varepsilon}_{2}\right)\end{aligned}$ \\
\hline $\begin{array}{l}\Gamma_{1}^{t}<\Gamma_{2}^{ \pm} \\
\Gamma_{3}^{ \pm x} \Gamma_{4}^{ \pm}\end{array}$ & $\begin{array}{l}K_{1} \leftrightarrow K_{3} \\
K_{2} \leftrightarrow K_{4}\end{array}$ & $\begin{array}{l}\Delta_{1} \leftrightarrow \Delta_{3} \\
\Delta_{2} \leftrightarrow \Delta_{4}\end{array}$ & $\left(I_{1} m_{2}+I_{2} m_{1}\right)^{2} \equiv A_{2}\left(\hat{\varepsilon}_{1} \hat{\varepsilon}_{2}\right)$ \\
\hline $\begin{array}{l}\Gamma_{1}^{ \pm} \leftrightarrow \Gamma_{3}^{ \pm} \\
\Gamma_{1}^{ \pm} \leftrightarrow \Gamma_{4}^{ \pm} \\
\Gamma_{2}^{ \pm} \leftrightarrow \Gamma_{3}^{ \pm} \\
\Gamma_{2}^{ \pm} \leftrightarrow \Gamma_{4}^{ \pm}\end{array}$ & $\begin{array}{l}\mathrm{K}_{1} \leftrightarrow \mathrm{K}_{2} \\
\mathrm{I}_{1} \leftrightarrow \mathrm{K}_{1} \\
\mathrm{~K}_{3} \leftrightarrow \mathrm{K}_{2} \\
\mathrm{~K}_{3} \leftrightarrow \mathrm{K}_{4}\end{array}$ & $\begin{array}{l}\Delta_{1} \leftrightarrow \Delta_{2} \\
\Delta_{1} \leftrightarrow \Delta_{4} \\
\Delta_{3} \leftrightarrow \Delta_{2} \\
\Delta_{3} \leftrightarrow \Delta_{4}\end{array}$ & forbidden \\
\hline
\end{tabular}


Table 3-3 (Contd.)

\begin{tabular}{|c|c|c|c|}
\hline$\Gamma$ & $\mathrm{K}$ & $\triangle$ & \\
\hline $\begin{array}{l}\Gamma_{1}^{ \pm} \leftrightarrow \Gamma_{6}^{ \pm} \\
\Gamma_{2}^{ \pm} \leftrightarrow \Gamma_{6}^{ \pm} \\
\Gamma_{3}^{ \pm} \leftrightarrow \Gamma_{5}^{*} \\
\Gamma_{4}^{ \pm} \leftrightarrow \Gamma_{5}^{2} \\
\Gamma_{5}^{ \pm} \leftrightarrow \Gamma_{6}^{2}\end{array}$ & $\begin{array}{l}\mathrm{K}_{1} \leftrightarrow \mathrm{K}_{6} \\
\mathrm{~K}_{3} \leftrightarrow \mathrm{K}_{6} \\
\mathrm{~K}_{2} \leftrightarrow \mathrm{K}_{5} \\
\mathrm{~K}_{4} \leftrightarrow \mathrm{K}_{5} \\
\mathrm{~K}_{5} \leftrightarrow \mathrm{K}_{6}\end{array}$ & $\begin{array}{l}\Delta_{1} \leftrightarrow \Delta_{6} . \\
\Delta_{3} \leftrightarrow \Delta_{6} \\
\Delta_{2} \leftrightarrow \Delta_{5} \\
\Delta_{4} \leftrightarrow \Delta_{5} \\
\Delta_{5} \leftrightarrow \Delta_{6}\end{array}$ & $\begin{array}{r}{\left[\left(m_{1} n_{2}-m_{2} n_{1}\right)+\lambda_{2}\left(m_{1} n_{2}+m_{2} n_{1}\right)\right]^{2}} \\
{\left[\left(n_{1} I_{2}-n_{2} I_{1}\right)-\lambda_{2}\left(n_{1} I_{2}+n_{2} I_{1}\right)\right]^{2}} \\
\equiv F_{1}\left(\hat{\varepsilon}_{1} \hat{\varepsilon}_{2}\right)\end{array}$ \\
\hline $\begin{array}{l}\Gamma_{1}^{ \pm} \leftrightarrow \Gamma_{5}^{ \pm} \\
\Gamma_{2}^{ \pm} \leftrightarrow \Gamma_{5}^{ \pm} \\
\Gamma_{3}^{ \pm} \leftrightarrow \Gamma_{6}^{ \pm} \\
\Gamma_{4}^{ \pm} \leftrightarrow \Gamma_{6}^{ \pm}\end{array}$ & $\begin{array}{l}\mathrm{K}_{1} \leftrightarrow \mathrm{K}_{5} \\
\mathrm{~K}_{3} \leftrightarrow \mathrm{K}_{5} \\
\mathrm{~K}_{2} \leftrightarrow \mathrm{K}_{6} \\
\mathrm{~K}_{4} \leftrightarrow \mathrm{F}_{6}\end{array}$ & $\begin{array}{l}\Delta_{1} \leftrightarrow \Delta_{5} \\
\Delta_{3} \leftrightarrow \Delta_{5} \\
\Delta_{2} \leftrightarrow \Delta_{6} \\
\Delta_{4} \leftrightarrow \Delta_{6}\end{array}$ & $\left(1-n_{1}^{2}\right)\left(1-n_{2}^{2}\right) \equiv \mathbb{E}_{2}\left(\hat{\varepsilon}_{1} \hat{\varepsilon}_{2}\right)$ \\
\hline $\begin{array}{l}\Gamma_{5}^{ \pm} \leftrightarrow \Gamma_{5}^{ \pm} \\
\Gamma_{6}^{ \pm} \leftrightarrow \Gamma_{6}^{ \pm}\end{array}$ & $\begin{array}{l}K_{5} \leftrightarrow K_{5} \\
K_{6} \leftrightarrow K_{6}\end{array}$ & $\begin{array}{l}\Delta_{5} \leftrightarrow \Delta_{5} \\
\Delta_{6} \leftrightarrow \Delta_{6}\end{array}$ & $A_{1}\left(\hat{\varepsilon}_{1} \hat{\varepsilon}_{2}\right)+c_{1} A_{2}\left(\hat{\varepsilon}_{1} \hat{\varepsilon}_{2}\right)+c_{2} E_{2}\left(\hat{\varepsilon}_{1} \hat{\varepsilon}_{2}\right)$ \\
\hline $\begin{array}{l}\Gamma_{7}^{ \pm} \leftrightarrow \Gamma_{7}^{ \pm} \\
\Gamma_{8}^{ \pm} \leftrightarrow \Gamma_{8}^{ \pm}\end{array}$ & $\begin{array}{l}\mathrm{K}_{7} \leftrightarrow \mathrm{K}_{7} \\
\mathrm{~K}_{8} \leftrightarrow \mathrm{K}_{8}\end{array}$ & $\begin{array}{l}\Delta_{7} \leftrightarrow \Delta_{7} \\
\Delta_{8} \leftrightarrow \Delta_{8}\end{array}$ & $A_{1}\left(\hat{\varepsilon}_{1} \hat{\varepsilon}_{2}\right)+c_{1} A_{2}\left(\hat{\varepsilon}_{1} \hat{\varepsilon}_{2}\right)+c_{2} E_{1}\left(\hat{\varepsilon}_{1} \hat{\varepsilon}_{2}\right)$ \\
\hline
\end{tabular}


Table 3-3 (Contd.)

\begin{tabular}{|c|c|c|c|}
\hline & $K$ & $\Delta$ & \\
\hline$\Gamma_{7}^{ \pm} \leftrightarrow \Gamma_{8}^{ \pm}$ & $K_{7} \leftrightarrow K_{8}$ & $\Delta_{7} \leftrightarrow \Delta_{8}$ & $E_{2}\left(\hat{\varepsilon}_{1} \hat{\varepsilon}_{2}\right)$ \\
\hline$\Gamma_{7}^{ \pm} \leftrightarrow \Gamma_{9}^{ \pm}$ & $K_{7} \leftrightarrow K_{9}$ & $\Delta_{7} \leftrightarrow \Delta_{9}$ & $E_{1}\left(\hat{\varepsilon}_{1} \hat{\varepsilon}_{2}\right)+c E_{2}\left(\hat{\varepsilon}_{1} \hat{\varepsilon}_{2}\right)$ \\
$\Gamma_{8}^{ \pm} \leftrightarrow \Gamma_{9}^{ \pm \pm}$ & $K_{8} \leftrightarrow K_{9}$ & $\Delta_{8} \leftrightarrow \Delta_{9}$ & $\vdots$ \\
\hline$\Gamma_{9}^{ \pm} \leftrightarrow \Gamma_{9}^{ \pm}$ & $K_{9} \leftrightarrow K_{9}$ & $\Delta_{9} \leftrightarrow \Delta_{9}$ & $A_{1}\left(\hat{\varepsilon}_{1} \hat{\varepsilon}_{2}\right)+c A_{2}\left(\hat{\varepsilon}_{1} \hat{\varepsilon}_{2}\right)$ \\
\hline
\end{tabular}

\begin{tabular}{|c|c|c|}
\hline$A$ & $\mathrm{H}$ & \\
\hline $\mathrm{A}_{1} \leftrightarrow A_{1}$ & $\left(\mathrm{H}_{5} \mathrm{H}_{7}\right) \leftrightarrow\left(\mathrm{H}_{5} \mathrm{H}_{7}\right)$ & $\mathrm{A}_{I}\left(\hat{\varepsilon}_{1} \hat{\varepsilon}_{2}\right)$ \\
\hline $\mathrm{A}_{1} \leftrightarrow A_{2}$ & & $\mathrm{~A}_{2}\left(\hat{\varepsilon}_{1} \hat{\varepsilon}_{2}\right)$ \\
\hline$\left(\mathrm{A}_{4} \mathrm{~A}_{5}\right) \leftrightarrow\left(\mathrm{A}_{4} \mathrm{~A}_{5}\right)$ & $\begin{array}{c}\mathrm{H}_{1} \leftrightarrow \mathrm{H}_{1} \\
\left(\mathrm{H}_{4} \mathrm{H}_{6}\right) \leftrightarrow\left(\mathrm{H}_{4} \mathrm{H}_{6}\right)\end{array}$ & $\mathrm{A}_{1}\left(\hat{\varepsilon}_{1} \hat{\varepsilon}_{2}\right)+\mathrm{cA}_{2}\left(\hat{\varepsilon}_{1} \hat{\varepsilon}_{2}\right)$ \\
\hline & $\begin{array}{c}\mathrm{H}_{8} \leftrightarrow \mathrm{H}_{9} \\
\left(\mathrm{H}_{4} \mathrm{H}_{6}\right) \leftrightarrow \mathrm{H}_{8} \\
\left(\mathrm{H}_{5} \mathrm{H}_{7}\right) \leftrightarrow \mathrm{H}_{9}\end{array}$ & $\mathrm{E}_{1}\left(\hat{\varepsilon}_{1} \hat{\varepsilon}_{2}\right)$ \\
\hline
\end{tabular}


Table 3-3 (Conta.)

\begin{tabular}{|c|c|c|}
\hline \multirow[t]{2}{*}{ A } & $\mathrm{H}$ & \\
\hline & $\begin{aligned} \mathrm{H}_{2} & \leftrightarrow \mathrm{H}_{3} \\
\left(\mathrm{H}_{4} \mathrm{H}_{6}\right) & \leftrightarrow \mathrm{H}_{9} \\
\left(\mathrm{H}_{5} \mathrm{H}_{7}\right) & \leftrightarrow \mathrm{H}_{8}\end{aligned}$ & $E_{2}\left(\hat{\varepsilon}_{1} \hat{\varepsilon}_{2}\right)$ \\
\hline \multirow{2}{*}{$\begin{aligned}\left(A_{4} A_{5}\right) & \leftrightarrow A_{6} . \\
A_{1} & \leftrightarrow A_{3} \\
A_{2} & \leftrightarrow A_{3}\end{aligned}$} & $\mathrm{H}_{1} \longleftrightarrow \mathrm{H}_{2}$ & $E_{1}\left(\hat{\varepsilon}_{1} \hat{\varepsilon}_{2}\right)+c_{1} E_{2}\left(\hat{\varepsilon}_{1} \hat{\varepsilon}_{2}\right)$ \\
\hline & $\begin{array}{l}\mathrm{H}_{2} \leftrightarrow \mathrm{H}_{2} \\
\mathrm{H}_{8} \leftrightarrow \mathrm{H}_{8} \\
\mathrm{H}_{9} \leftrightarrow \mathrm{H}_{9}\end{array}$ & $A_{1}\left(\hat{\varepsilon}_{1} \hat{\varepsilon}_{2}\right)+c_{1} A_{2}\left(\hat{\varepsilon}_{1} \hat{\varepsilon}_{2}\right)+C_{2} E_{1}\left(\hat{\varepsilon}_{1} \hat{\varepsilon}_{2}\right)$ \\
\hline \multirow[t]{2}{*}{$\begin{array}{l}A_{3} \leftrightarrow A_{3} \\
A_{6} \leftrightarrow A_{6}\end{array}$} & & $\mathrm{~A}_{1}\left(\hat{\varepsilon}_{1} \hat{\varepsilon}_{2}\right)+\mathrm{c}_{1} \mathrm{~A}_{2}\left(\hat{\varepsilon}_{1} \hat{\varepsilon}_{2}\right)+\mathrm{c}_{2} \mathrm{E}_{1}\left(\hat{\varepsilon}_{1} \hat{\varepsilon}_{2}\right)+\mathrm{c}_{3} \mathrm{E}_{2}\left(\hat{\varepsilon}_{1} \hat{\varepsilon}_{2}\right)$ \\
\hline & $\left(\mathrm{H}_{4} \mathrm{H}_{6}\right) \leftrightarrow\left(\mathrm{H}_{5} \mathrm{H}_{7}\right)$ & forbidaen \\
\hline
\end{tabular}


Table 3-3 (Contd。)

\begin{tabular}{|c|c|c|c|c|c|}
\hline$M^{\circ}$ & $\Sigma$ & $\mathrm{R}$ & U & $\mathrm{T}$ & \\
\hline $\begin{array}{l}M_{2}^{ \pm} \leftrightarrow M_{1}^{ \pm} \\
M_{2}^{ \pm} \leftrightarrow M_{2}^{ \pm} \\
M_{3}^{ \pm} \leftrightarrow M_{3}^{ \pm} \\
M_{4}^{ \pm} \leftrightarrow M_{4}^{2} .\end{array}$ & $\begin{array}{l}\Sigma_{1} \leftrightarrow \Sigma_{1} \\
\Sigma_{2} \leftrightarrow \Sigma_{2} \\
\Sigma_{3} \leftrightarrow \Sigma_{3} \\
\Sigma_{4} \leftrightarrow \Sigma_{4}\end{array}$ & $\begin{array}{l}R_{1} \leftrightarrow R_{1} \\
R_{2} \leftrightarrow R_{2} \\
R_{3} \leftrightarrow R_{3} \\
R_{4} \leftrightarrow R_{4}\end{array}$ & $\begin{array}{l}U_{1} \leftrightarrow U_{1} \\
U_{2} \leftrightarrow U_{2} \\
U_{3} \leftrightarrow U_{3} \\
U_{4} \leftrightarrow U_{4}\end{array}$ & $\begin{array}{l}T_{1} \leftrightarrow T_{1} \\
T_{2} \leftrightarrow T_{2} \\
T_{3} \leftrightarrow T_{3} \\
T_{4} \leftrightarrow T_{4} .\end{array}$ & $A_{1}\left(\hat{\varepsilon}_{1} \hat{\varepsilon}_{2}\right)+c \mathbb{F}_{2}\left(\hat{\varepsilon}_{1} \hat{\varepsilon}_{2}\right)$ \\
\hline $\begin{array}{l}M_{1}^{ \pm} \leftrightarrow M_{2}^{ \pm} \\
M_{1}^{ \pm} \leftrightarrow M_{3}^{ \pm} \\
M_{2}^{ \pm} \leftrightarrow M_{4}^{ \pm} \\
M_{3}^{ \pm} \leftrightarrow M_{4}^{ \pm}\end{array}$ & $\begin{array}{l}\Sigma_{1} \leftrightarrow \Sigma_{2} \\
\Sigma_{1} \leftrightarrow \Sigma_{3} \\
\Sigma_{2} \leftrightarrow \Sigma_{4} \\
\Sigma_{3} \leftrightarrow \Sigma_{4}\end{array}$ & $\begin{array}{l}R_{1} \leftrightarrow R_{2} \\
R_{1} \leftrightarrow R_{3} \\
R_{2} \leftrightarrow R_{4} \\
R_{3} \leftrightarrow R_{4}\end{array}$ & $\begin{array}{l}U_{1} \leftrightarrow U_{2} \\
U_{1} \leftrightarrow U_{3} \\
U_{2} \longleftrightarrow U_{4} \\
U_{3} \leftrightarrow U_{4}\end{array}$ & $\begin{array}{l}T_{1} \leftrightarrow T_{2} \\
T_{1} \leftrightarrow T_{3} \\
T_{2} \leftrightarrow T_{4} \\
T_{3} \leftrightarrow T_{4}\end{array}$ & $E_{1}\left(\hat{\varepsilon}_{1} \hat{\varepsilon}_{2}\right)$ \\
\hline $\begin{array}{l}M_{2}^{2} \leftrightarrow M_{4}^{ \pm} \\
M_{2}^{2} \leftrightarrow M_{3}^{ \pm}\end{array}$ & $\begin{array}{l}\Sigma_{1} \leftrightarrow \Sigma_{4} \\
\Sigma_{2} \leftrightarrow \Sigma_{3}\end{array}$ & $\begin{array}{l}R_{1} \leftrightarrow R_{4} \\
R_{2} \leftrightarrow R_{3}\end{array}$ & $\begin{array}{l}\mathrm{U}_{1} \leftrightarrow \mathrm{U}_{4} \\
\mathrm{U}_{2} \leftrightarrow \mathrm{U}_{3}\end{array}$ & $\begin{array}{l}\mathrm{T}_{1} \leftrightarrow \mathrm{T}_{4} \\
\mathrm{~T}_{2} \leftrightarrow \mathrm{T}_{3}\end{array}$ & $A_{2}\left(\hat{\varepsilon}_{1} \hat{\varepsilon}_{2}\right)+c E_{2}\left(\hat{\varepsilon}_{1} \hat{\varepsilon}_{2}\right)$ \\
\hline$M_{5}^{2} \leftrightarrow M_{5}^{2}$ & $\Sigma^{5} \leftrightarrow \Sigma_{5}$ & $R_{5} \leftrightarrow R_{5}$ & $U_{5} \leftrightarrow U_{5}$ & $T_{5} \leftrightarrow T_{5}$ & $\begin{aligned} & A_{1}\left(\hat{\varepsilon}_{1} \hat{\varepsilon}_{2}\right)+c_{1} A_{2}\left(\hat{\varepsilon}_{1} \hat{\varepsilon}_{2}\right) \\
+ & c_{2} E_{1}\left(\hat{\varepsilon}_{1} \hat{\varepsilon}_{2}\right)+c_{3} E_{2}\left(\hat{\varepsilon}_{I} \hat{\varepsilon}_{2}\right)\end{aligned}$ \\
\hline
\end{tabular}


Table 3-3 (Contd.)

\begin{tabular}{|c|c|}
\hline$I$ & $A_{I}\left(\hat{\varepsilon}_{I} \hat{\varepsilon}_{2}\right)+c_{I} E_{I}\left(\hat{\varepsilon}_{I} \hat{\varepsilon}_{2}\right)+c_{2} F_{2}\left(\hat{\varepsilon}_{I} \hat{\varepsilon}_{2}\right)$ \\
\hline$I_{I} \leftrightarrow I_{I}$ & $A_{2}\left(\hat{\varepsilon}_{I} \hat{\varepsilon}_{2}\right)+c_{I} E_{I}\left(\hat{\varepsilon}_{I} \hat{\varepsilon}_{2}\right)+c_{2} E_{2}\left(\hat{\varepsilon}_{I} \hat{\varepsilon}_{2}\right)$ \\
\hline$\left(I_{3} I_{4}\right) \leftrightarrow\left(I_{3} I_{4}\right)$ & $A_{I}\left(\hat{\varepsilon}_{I} \hat{\varepsilon}_{2}\right)+c_{I} A_{2}\left(\hat{\varepsilon}_{I} \hat{\varepsilon}_{2}\right)+c_{3} E_{I}\left(\hat{\varepsilon}_{I} \hat{\varepsilon}_{2}\right)+c_{4} E_{2}\left(\hat{\varepsilon}_{I} \hat{\varepsilon}_{2}\right)$ \\
\hline
\end{tabular}

\begin{tabular}{|c|c|}
\hline$S_{1} \leftrightarrow S_{1}$ & $A_{1}\left(\hat{\varepsilon}_{1} \hat{\varepsilon}_{2}\right)+c_{1} A_{2}\left(\hat{\varepsilon}_{1} \hat{\varepsilon}_{2}\right)+c_{2} E_{1}\left(\hat{\varepsilon}_{1} \hat{\varepsilon}_{2}\right)+c_{3} E_{2}\left(\hat{\varepsilon}_{1} \hat{\varepsilon}_{2}\right)$ \\
\hline $\begin{array}{l}\left(S_{3} S_{4}\right)-\left(S_{3} S_{4}\right) \\
\left(S_{2} S_{5}\right)-\left(S_{2} S_{5}\right)\end{array}$ & $A_{1}\left(\hat{\varepsilon}_{1} \hat{\varepsilon}_{2}\right)+c_{1} A_{2}\left(\hat{\varepsilon}_{1} \hat{\varepsilon}_{2}\right)+c_{2} E_{2}\left(\hat{\varepsilon}_{1} \hat{\varepsilon}_{2}\right)$ \\
\hline$\left(S_{3} S_{4}\right)-\left(S_{2} S_{5}\right)$ & $E_{1}\left(\hat{\varepsilon}_{1} \hat{\varepsilon}_{2}\right)$ \\
\hline
\end{tabular}


Table 3-3 (Contd.)

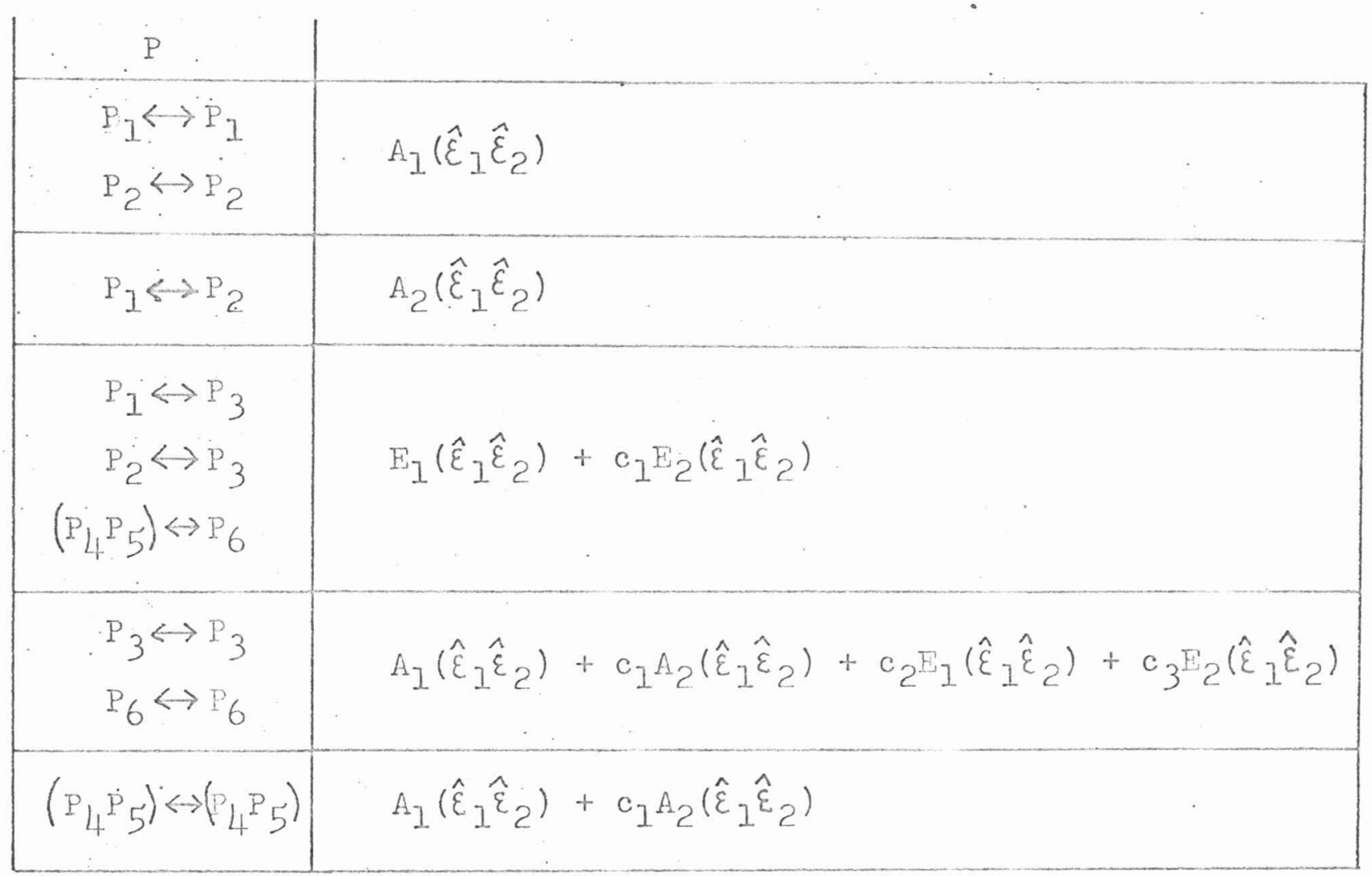

$\rightleftarrows$ 


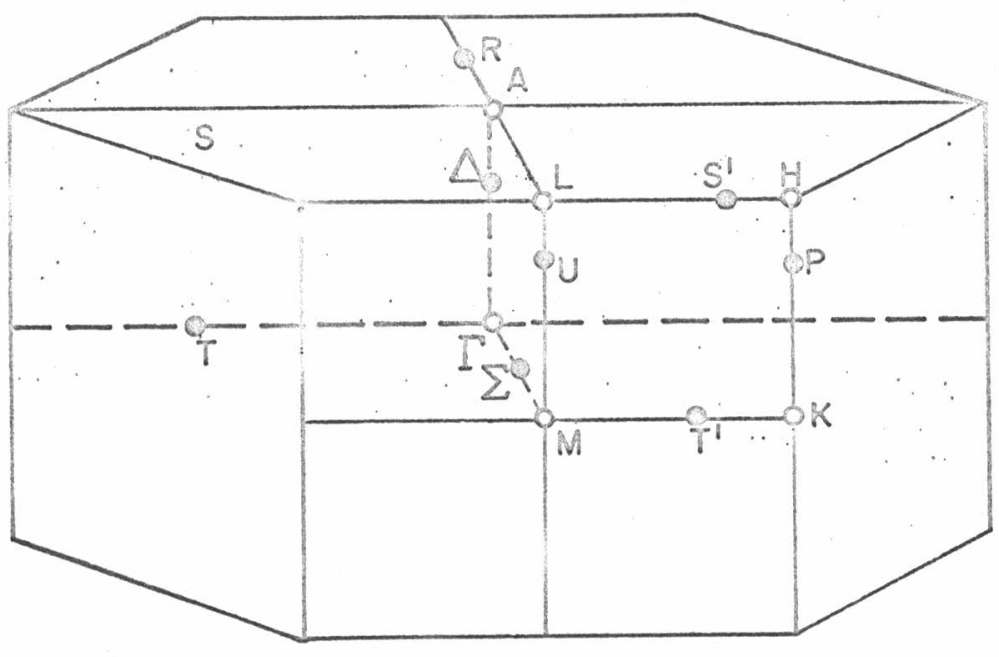

Fig.3-5. Brillouin zone of hexagonal close-packed and wurtite structures 
Table $3-4$

Angular dependence functions for wurtzite structuras. Notations used are those of Glasser (Ref.47) and of Casella (Ref.48).

\begin{tabular}{|c|c|c|c|}
$\Gamma$ & $\Delta$ & $A$ & \\
\hline$\Gamma_{1} \leftrightarrow \Gamma_{1}$ & $\Delta_{1} \leftrightarrow \Delta_{1}$ & $\left(\Delta_{1} A_{3}\right) \leftrightarrow\left(A_{1} A_{3}\right)$ & \\
$\Gamma_{2} \leftrightarrow \Gamma_{2}$ & $\Delta_{2} \leftrightarrow \Delta_{2}$ & $\left(A_{2} A_{4}\right) \leftrightarrow\left(A_{2} A_{4}\right)$ & {$\left[\left(I_{1} I_{2}+m_{I} m_{2}\right)+\lambda_{I_{1}} n_{2}\right]^{2} \equiv A_{1}\left(\hat{\varepsilon}_{1} \hat{\varepsilon}_{2}\right)$} \\
$\Gamma_{3} \leftrightarrow \Gamma_{3}$ & $\Delta_{3} \leftrightarrow \Delta_{3}$ & & \\
$\Gamma_{4} \leftrightarrow \Gamma_{4}$ & $\Delta_{4} \leftrightarrow \Delta_{4}$ & & $\left(I_{1} m_{2}-I_{2} m_{1}\right)^{2} \equiv A_{2}\left(\hat{\varepsilon}_{1} \hat{\varepsilon}_{2}\right)$ \\
\hline$\Gamma_{1} \leftrightarrow \Gamma_{3}$ & $\Delta_{1} \leftrightarrow \Delta_{3}$ & & \\
$\Gamma_{2} \leftrightarrow \Gamma_{4}$ & $\Delta_{2} \leftrightarrow \Delta_{4}$ & & forbidden \\
\hline$\Gamma_{1} \leftrightarrow \Gamma_{2}$ & $\Delta_{1} \leftrightarrow \Delta_{2}$ & $\left(A_{1} A_{3}\right) \leftrightarrow\left(\Delta_{2} A_{4}\right)$ & \\
$\Gamma_{1} \leftrightarrow \Gamma_{4}$ & $\Delta_{1} \leftrightarrow \Delta_{4}$ & & \\
$\Gamma_{2} \leftrightarrow \Gamma_{3}$ & $\Delta_{2} \leftrightarrow \Delta_{3}$ & & \\
$\Gamma_{3} \leftrightarrow \Gamma_{4}$ & $\Delta_{3} \leftrightarrow \Delta_{4}$ & & \\
\hline
\end{tabular}




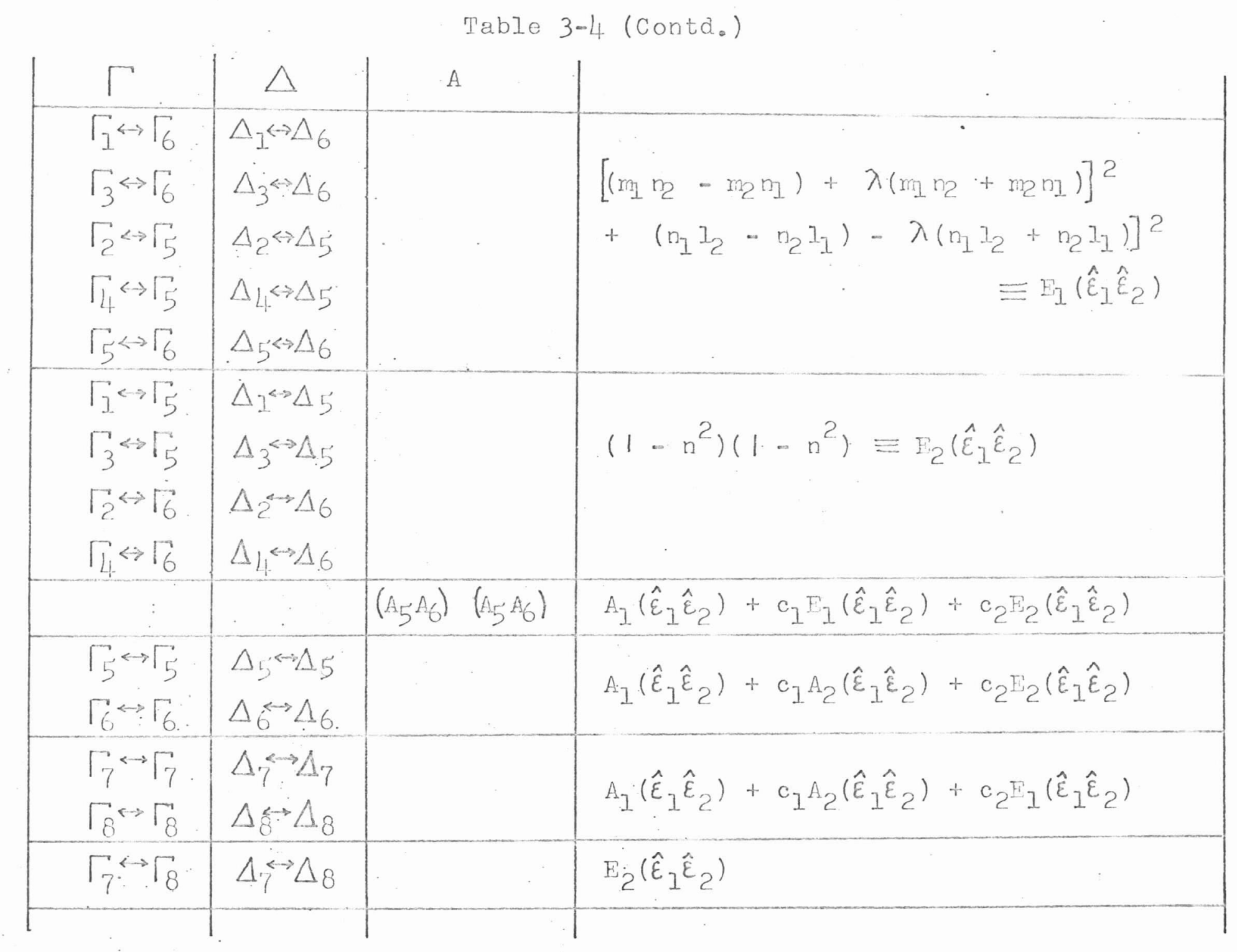


Table 3-4 (Conta.)

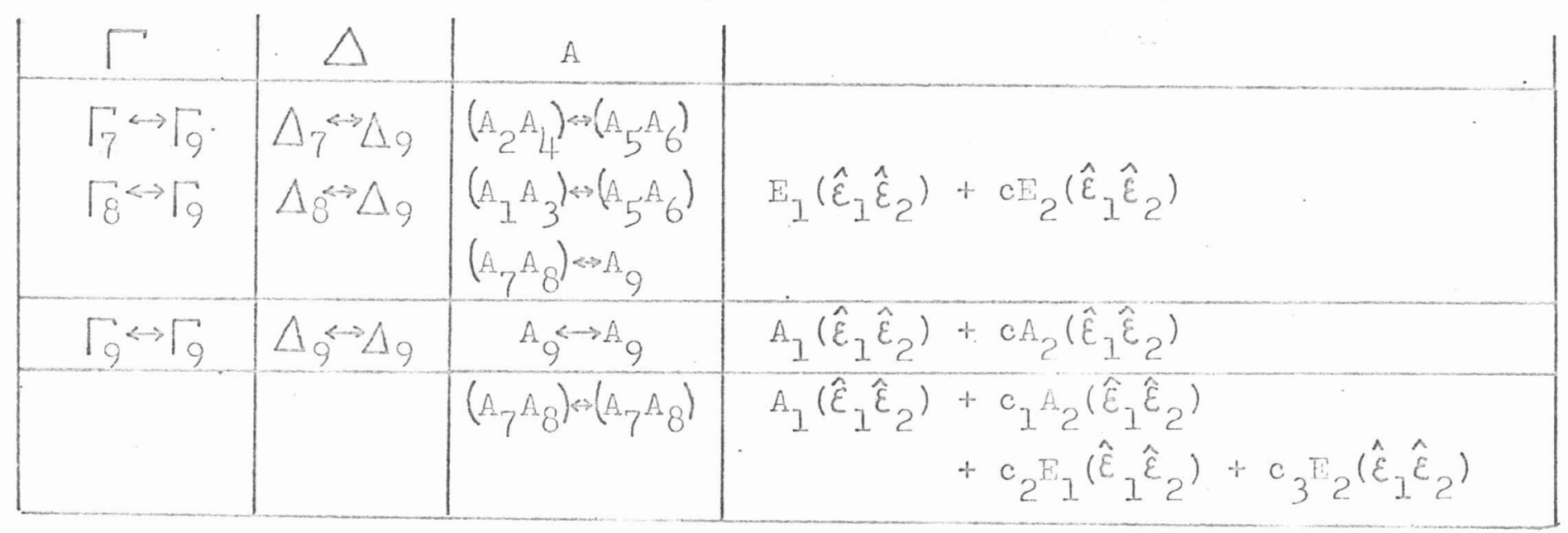

\begin{tabular}{|c|c|c|c|}
$\mathrm{K}$ & $\mathrm{P}$ & \\
\hline & $\mathrm{K}_{1} \leftrightarrow \mathrm{K}_{1}$ & $\mathrm{P}_{1} \leftrightarrow \mathrm{P}_{1}$ & \\
$\mathrm{~K}_{2} \leftrightarrow \mathrm{K}_{2}$ & $\mathrm{P}_{2} \leftrightarrow \mathrm{P}_{2}$ & $\mathrm{~A}_{1}\left(\hat{\varepsilon}_{1} \hat{\varepsilon}_{2}\right)$ \\
$\mathrm{K}_{4} \leftrightarrow \mathrm{K}_{4}$ & $\mathrm{P}_{4} \leftrightarrow \mathrm{P}_{4}$ & \\
& $\mathrm{~K}_{5} \leftrightarrow \mathrm{K}_{5}$ & $\mathrm{P}_{5} \leftrightarrow \mathrm{P}_{5}$ & \\
\hline $\mathrm{K}_{1} \leftrightarrow \mathrm{K}_{2}$ & $\mathrm{P}_{1} \leftrightarrow \mathrm{P}_{2}$ & $\mathrm{~A}_{2}\left(\hat{\varepsilon}_{1} \hat{\varepsilon}_{2}\right)$ \\
& $\mathrm{K}_{4} \leftrightarrow \mathrm{K}_{5}$ & $\mathrm{P}_{4} \leftrightarrow \mathrm{P}_{5}$ & ${ }$ \\
& &
\end{tabular}

$\vec{\infty}$ 
Table 3-4 (Conta.)

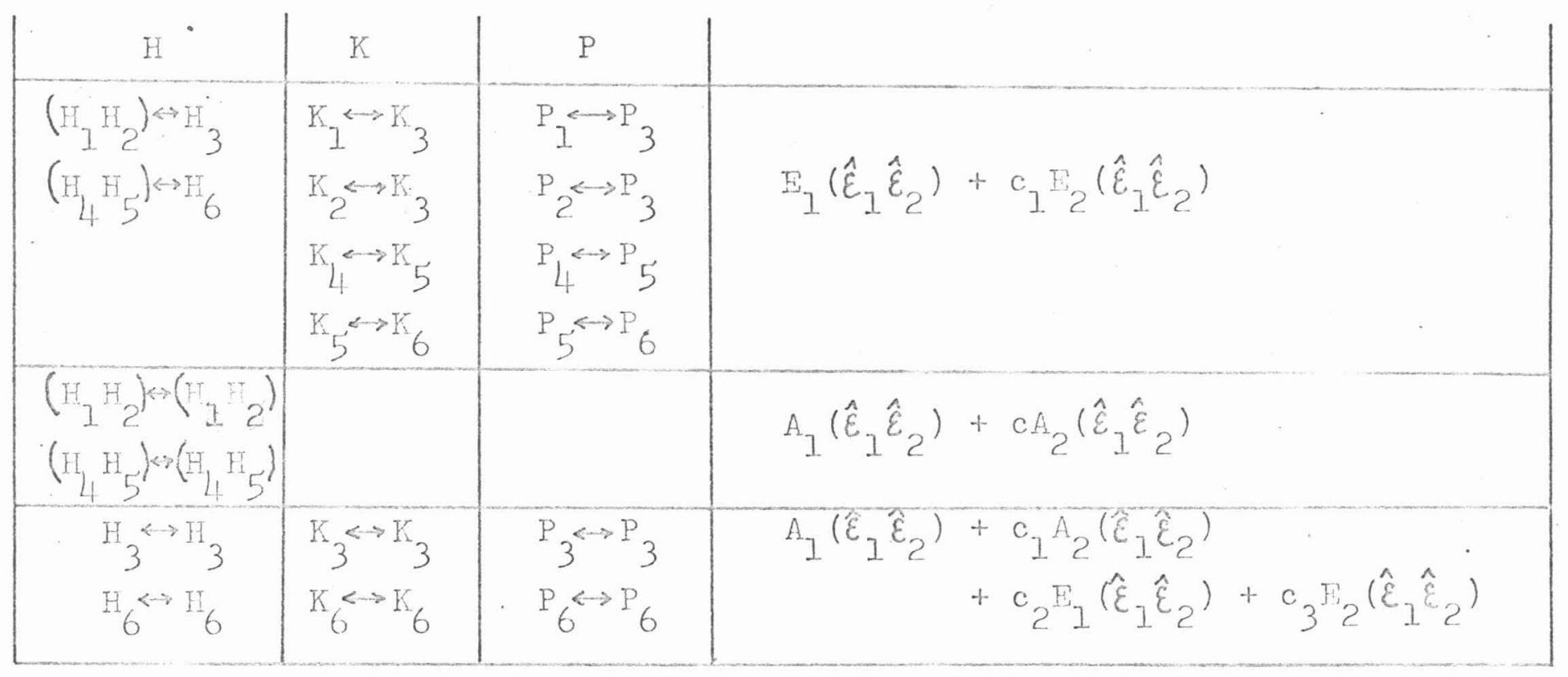

ชื 


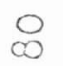

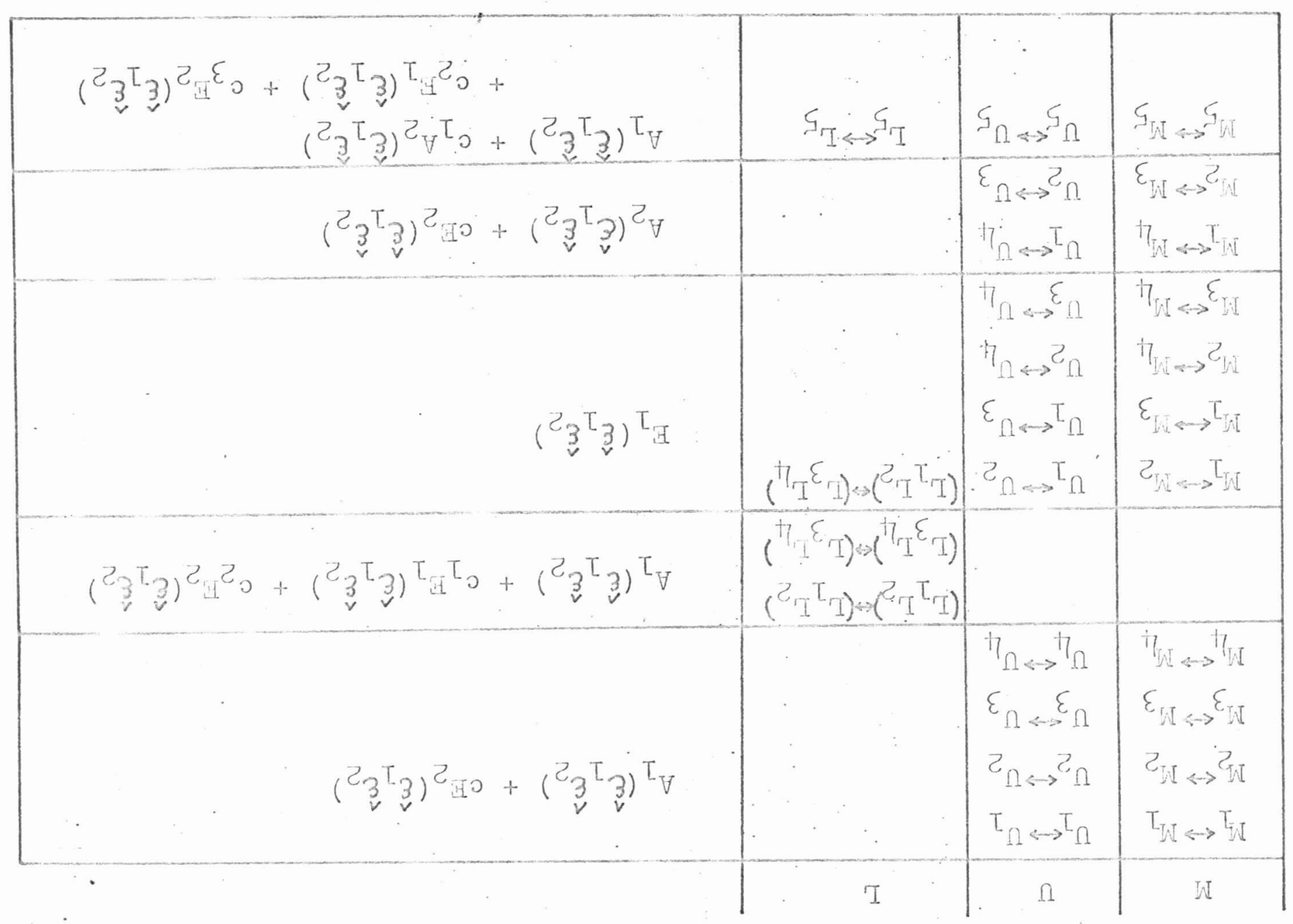

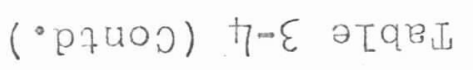


Table 3-4 (Contd.)

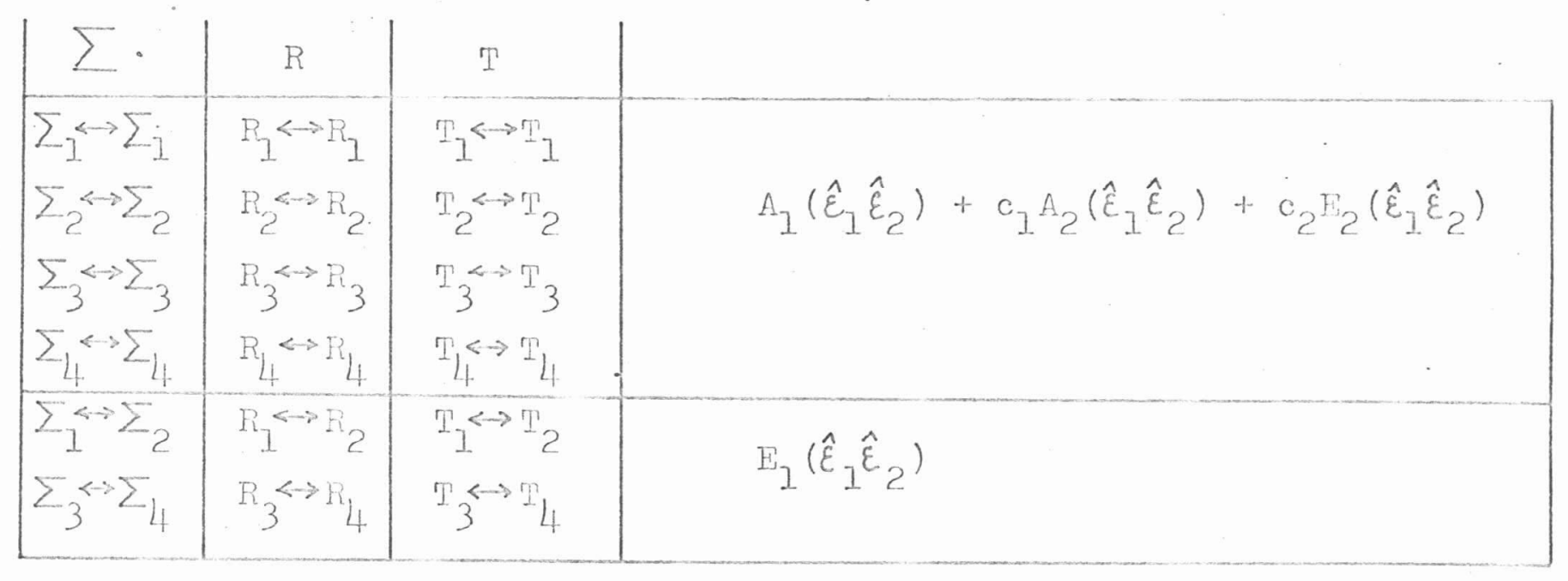

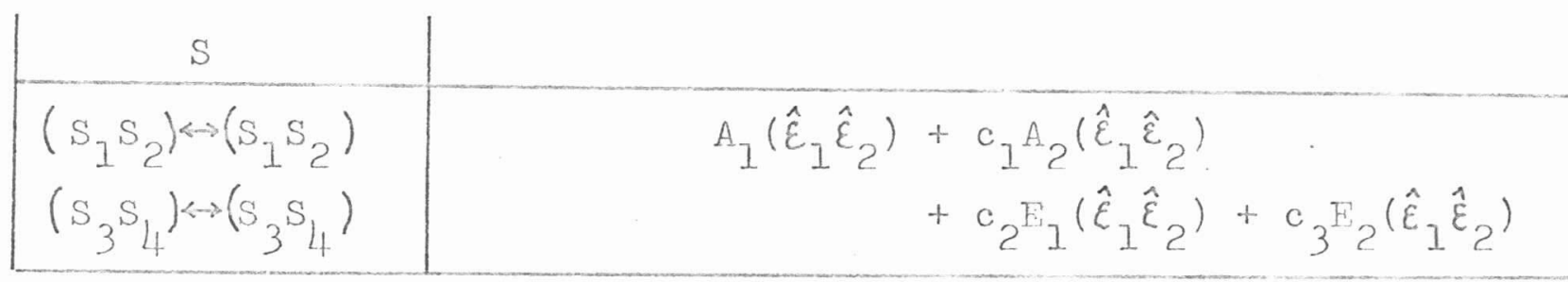

$\infty$ 


\section{Chapter IV}

\section{Forbidden Transitions}

In many crystals of interest, such as the alkali halides, in which the point group contains the inversion, the valence band and conduction band at a high symmetry point often have opposite parity. This means that the dependence of the absorption coefficient on the directions of polarization will not be given by the tables in Chapter II for transitions taking place near extrema occurring at these points. In this section we will discuss Mahan's calculations of the angular dependence in a spherical band model with the explicit use of hydrogenic excitons in the initial and final states. A less sophisticated calculation will then be made in which the electron-hole interaction is ignored, and this will be compared with the calculation of Mahan. In this simpler model some refinements can be made more easily. Two of these will be discussed: the special case of valence p-bands near $\vec{k}=0$ split into a spherical non-degenerate band and a spherical two-fold degenerate band, and ellipsoidal energy surfaces at point $X$ for simple cubic and face-centered cubic crystals. 


\section{A. Mahan's Model}

As was noted after Eq.2-23, for a forbidden two-photon transition the final state exciton envelope must, in the hydrogenic model, be a p-state. Its symmetry is determined by the decomposition of the $l=1$ representation of the full rotation group into representations of the crystal. point group. If an envelope function transforms as $\Gamma^{\alpha}$, and if $\left|c_{i}\left(k_{0}\right)\right\rangle$ and $\left|v_{j}\left(k_{0}\right)\right\rangle$ transform as $\Gamma^{c}$ and $\Gamma^{v}$, exciton states can be formed 49,50 which transform as representations contained in the decomposition of $\Gamma^{\alpha} \times \Gamma^{c} \times \Gamma^{v}$. Suppose a p-state in a crystal field decomposes according to $D^{\prime}=\sum_{\alpha} \Gamma^{\alpha}$. Then the final state excitons for forbidden transitions will transform according to $\sum_{\lambda \tau_{\lambda}} \Gamma^{\lambda \tau_{\lambda}}=\sum_{\alpha} \Gamma^{\alpha} \times \Gamma^{c} \times \Gamma^{v}$, where, as in Chapter III, $\tau_{\lambda}$ is an index to account for the fact that $\Gamma^{\lambda}$ might occur more than once in the decomposition. If these states overlap, the angular dependence of the twophoton absorption will be given by

$$
\alpha^{(2)} \sim \sum_{\lambda \tau_{\lambda}} a^{\lambda \tau_{\lambda}} \gamma^{\lambda \tau_{\lambda}}\left(\hat{\varepsilon}_{1} \hat{\varepsilon}_{2}\right)
$$

where $a^{\lambda \tau_{\lambda}}$ are positive constants and $\gamma^{\lambda \tau_{\lambda}}$ are the functions of Table 3-1. In a real crystal all paths indicated in Eq.2-23 will contribute to the absorption. The problem now is to see if suitable approximations can be made to simplify Eq.4-I.

Referring to Eq.2-23, for $\vec{M}_{c_{i} n^{-}}$of the fourth line 
to be non-zero $n^{-}$must have the same parity as $c_{i} \cdot$ As an example of a band structure, consider that of $\mathrm{KI}^{5 I}$ shown in Fig.5-9 of Chapter V. As can be seen in this case there are no near conduction bands or valence bands (there will be core bands) with this property at $\Gamma$ or $\mathrm{x}$. $\vec{M}_{c_{i} n^{-}}$(or $\vec{M}_{n^{+} v_{j}}$ by the same argument) is expected, then, to be small compared with $\vec{M}_{c_{i} n^{+}}$(and $\vec{M}_{n^{-} v_{j}}$ ) due to larger energy denominators and smaller one-electron matrix elements. Also $E_{n^{+} v_{j}}(\nu)$ in the third line of Eq.2-23 will be larger than $E_{n^{+} v_{j}}(\nu)$ in the first line, since in the former case $n^{+}$represents higher conduction bands. Similarly $E_{c_{i} n^{-}}(\boldsymbol{\nu})$ of the fourth line will be larger than $E_{c_{i} n^{-}}(\nu)$ of the first line. When the band structure has the stated properties, then, we will neglect the third and fourth lines and their counterparts with subscripts $I$ and 2 permuted.

We will now, after Mahan, make the following assumptions: first, that $\vec{M}_{c_{i} n^{+}}$and $\vec{M}_{n^{-} v_{j}}$ of Eq.2-23 are sufficiently large when $n^{+}=c_{i}$ and $n^{-}=v_{j}$ so that terms due to other bands can be neglected; second, that the Wannier hydrogenic model is a good description of exciton states. The first assumption defines what will be referred to as a two band model, meaning that we can proceed with calculations in which the only bands needed are those contained in the manifolds $\left\{\left|c_{i}(k)\right\rangle\right\}$ and $\left\{\left|v_{j}(k)\right\rangle\right\}$, both of which are degenerate at the extremum, say at $\vec{k}=0$. The second 
assumption implies that surfaces of constant energy $E_{c_{i}}(k)-E_{v_{j}}(k)$ are spherical near $\vec{k}=0$. We must here, however, make the additional assumption not stated by Mahan that the bands $\left\{\left|c_{i}(k)\right\rangle\right\}$ remain degenerate and the bands $\left\{\left|V_{j}(k)\right\rangle\right\}$ remain degenerate near $\vec{k}=0$. Or, equivalently, the reduced reciprocal effective mass

$$
\left(\frac{1}{\mu_{i j}}\right)=\frac{1}{m}\left[\vec{M}_{c_{i} c_{i}}-\vec{M}_{v_{j} v_{j}}\right]
$$

is independent of $i$ and $j$.

Under these conditions only the first term in the sum over intermediate bands of $\mathrm{Eq}_{1} \cdot 2-23$, and its counterpart with subscripts 1 and 2 interchanged, contribute, with $\left|n^{+}\right\rangle=\left|c_{i}\right\rangle$ and $\left|n^{-}\right\rangle=\left|v_{j}\right\rangle$, giving the result

$$
\begin{aligned}
P_{f g}^{(2)} & = \\
& N^{\frac{1}{2}} \sum_{\nu} \hat{\varepsilon}_{1} \cdot\left(\frac{m}{\mu}\right) \cdot \frac{\left\langle u_{c v}^{\mu}|\vec{p}| u_{c v}^{\nu}\right\rangle u_{c v}^{\nu}(0)^{*}}{E_{c v}(\nu)-\hbar \omega_{2}}\left\langle c_{i}(0)\left|\hat{\varepsilon}_{2} \cdot \vec{p}\right| v_{j}(0)\right\rangle \\
+ & N^{\frac{1}{2}} \sum_{p} \hat{\varepsilon}_{2} \cdot\left(\frac{m}{\mu}\right) \cdot \frac{\left\langle u_{c v}^{\mu}|\vec{p}| u_{c v}^{\nu}\right\rangle u_{c v}^{\nu}(0)^{*}}{E_{c v}(\nu)-\hbar \omega_{1}}\left\langle c_{i}(0)\left|\hat{\varepsilon}_{1} \cdot \vec{p}\right| v_{j}(0)\right\rangle
\end{aligned}
$$

where we have dropped the subscripts $i$ and $j$ in the envelope matrix element and in $E_{c_{i} v_{j}}(\nu)$, since the equation that $\mathcal{U}_{c_{i} v_{j}}^{\nu}$ must satisfy $(E q .2-15)$ is independent of $i$ and $j$. Since the bands are spherical $(\overleftrightarrow{1 / \mu})$ is a constant $\frac{1}{\mu}$ times a unit tensor. Referring to Eq.2-24 we write the matrix element

$$
\left\langle u_{c v}^{\mu}|\vec{p}| u^{\nu}\right\rangle=\int \psi_{n l m}^{*}(\vec{\beta}) \vec{p} \psi_{n^{\prime} l^{\prime} m^{\prime}}(\vec{\beta}) d^{3} \beta
$$

where we have set $\mu=(n, I, m)$ and $\nu=\left(n^{\prime}, I^{\prime}, m^{\prime}\right)$ for hydrogenic excitons, and 


$$
\sqrt{\frac{N}{V}} U_{c v}^{\mu}(\vec{\beta}) \equiv \psi_{n \ell m}(\vec{\beta}) .
$$

From Eq.2-15 we see that $E_{c v}(p)=E_{g}+E_{n}$, where

$$
\begin{aligned}
& E_{n}=-\mu e^{4} / 2 \hbar^{2} n^{2} \cdot E q \cdot 4-2 \text { becomes }
\end{aligned}
$$

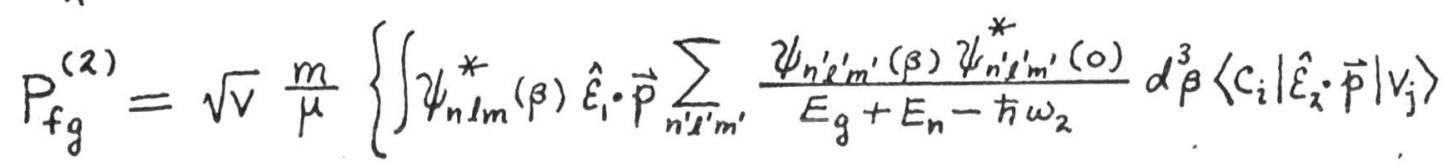

$$
\begin{aligned}
& \left.+\int \psi_{n l_{m}(\beta)}^{*} \hat{\varepsilon}_{2} \cdot \vec{p} \sum_{n^{\prime} \ell^{\prime} m^{\prime}} \frac{\psi_{n^{\prime} \ell^{\prime} m^{\prime}}(\beta) \psi_{n^{\prime} l^{\prime} m^{\prime}}^{*}(0)}{E_{g}+E_{n}-\hbar \omega_{1}} d^{3} \beta\left\langle c_{i}\left|\hat{\varepsilon}_{i} \cdot \vec{p}\right| v_{j}\right\rangle\right\}
\end{aligned}
$$

We have already said that since $\psi_{n^{\prime} \ell^{\prime} m^{\prime}}(0)$ is non-zero only for s-states $\psi_{n l m}$ must be a p-state. Mahan points out in addition that the sum over n'l'm' is the Coulomb Green's function

$$
\left.G_{\alpha}\left(\vec{\beta}, \vec{\beta}^{\prime}\right)\right|_{\beta^{\prime}=0}=\left.\sum_{n^{\prime} \prime^{\prime} m^{\prime}} \frac{\psi_{n^{\prime} \ell^{\prime} m^{\prime}}(\vec{\beta}) \psi_{n^{\prime} l^{\prime} m^{\prime}}^{*}\left(\vec{\beta}^{\prime}\right)}{E_{\lambda}-\Omega_{\alpha}}\right|_{\beta^{\prime}=0}
$$

where $\Omega_{\alpha} \equiv-E_{g}+\hbar \omega_{\alpha}$, and that this can be expressed in terms of a Whattaker's function,

$$
G_{\alpha}(\vec{\beta}, 0)=\frac{\mu}{2 \pi \hbar^{2} \beta} \Gamma\left(1-K_{\alpha}\right) W_{\kappa_{\alpha}, \frac{1}{2}}\left(\frac{2 \beta}{a K_{\alpha}}\right)
$$

where

$$
W_{k_{\alpha}, \frac{1}{2}}(z)=\frac{z e^{-z / 2}}{\Gamma\left(1-K_{\alpha}\right)} \int_{0}^{\infty} e^{-z t}\left(\frac{1+t}{t}\right)^{K_{\alpha}} d t
$$

$a$ is the exciton Bohr radius and $K_{\alpha} \equiv\left[E_{B} /\left(E_{g}-\hbar \omega_{\alpha}\right)\right]^{\frac{1}{2}}$.

$E_{B}$ is the exciton binding energy. The integrals in Eq.4-3 $c$ an then be evaluated numerically to any desired degree of approximation. It is convenient to put these integrals in the form 


$$
\begin{aligned}
I & =\frac{\hbar}{i} \int \psi_{n p_{m}}^{*}(\vec{\beta}) \hat{\mathcal{E}}_{j} \cdot \hat{\beta} \frac{\partial}{\partial \beta} G_{\alpha}(\vec{\beta}, 0) d^{3} \beta \quad(\gamma, \alpha=1, \lambda) \\
& =\frac{\hbar \varepsilon_{\gamma m}}{i E_{B} \sqrt{3 \pi a^{5}}} \sqrt{\frac{n^{2}-1}{n^{5}}} J_{p, n}\left(K_{\alpha}\right)
\end{aligned}
$$

for discrete p-states, where the numerically evaluated, integral is

$$
J_{p, n}\left(K_{\alpha}\right) \equiv \frac{K_{\alpha}^{2}}{2} \int_{0}^{\infty}\left(t+\frac{1}{2}\right)\left(\frac{1+t}{t}\right)^{K_{\alpha}} \frac{\left[t+\frac{1}{2}-\frac{K_{\alpha}}{2 n}\right]^{n-2}}{\left[t+\frac{1}{2}+\frac{K_{\alpha}}{2 n}\right]^{n+2}} d t .
$$

For continuum states

$$
I=\frac{(\hat{\varepsilon} \cdot \vec{k}) \hbar}{\sqrt{v} E_{B}} e^{\eta \frac{\pi}{2}}|\Gamma(2-i \eta)| J_{p, k}\left(K_{\alpha}\right),
$$

wi th

$$
J_{p, k}\left(K_{\alpha}\right) \equiv \frac{K_{\alpha}^{2}}{2} \int_{0}^{\infty}\left(t+\frac{1}{2}\right)\left(\frac{1+t}{t}\right)^{K_{\alpha}} \frac{\left[t+\frac{1}{2}-i \frac{K_{\alpha}}{2 \eta}\right]^{-i \eta-2}}{\left[t+\frac{1}{2}+i \frac{k_{\alpha}}{2 \eta}\right]^{i \eta-2}} d t
$$

where $E_{k}=-E_{B} / \eta^{2}$. Combining Eq.2-7, Eq.4-3 and Eq.4-4 or Eq. 4-5 one obtains the absorption coefficient for discrete or continuum states. The sum over final states in Eq.2-7 is

$$
\sum_{f}=\sum_{i j} \int d E_{f} p\left(E_{f}\right) \text {. }
$$

For a discrete state $\rho\left(E_{f}\right)$ is the normalized line shape function. 22 We assume here that the spectral band widths of the light beams are narrow compared with $\rho\left(E_{f}\right)$. For large n, where the states are close together and 
88

overlap, the density of states is

$$
\rho\left(E_{f}\right)=\left(\frac{\partial E_{n}}{\partial n}\right)^{-1}=\left[\frac{\partial}{\partial n}\left(-\frac{E_{B}}{n^{2}}\right)\right]^{-1}=\frac{n^{3}}{2 E_{B}} .
$$

For continuum states

$$
\sum_{f}=\sum_{i j} \frac{V}{(2 \pi)^{3}} \int d^{3} k \text {. }
$$

The absorption coefficient becomes

$$
\begin{aligned}
& \alpha^{(2)}=A\left(1-\frac{1}{n^{2}}\right) \frac{2 E_{B}}{n^{3}} \rho\left(E_{f}\right) \\
& (4-6) \\
& x \sum_{i j m}\left|\varepsilon_{1 m}\left\langle c_{i}\left|\hat{\varepsilon}_{2} \cdot \vec{p}\right| v_{j}\right\rangle J_{p, n}\left(\kappa_{1}\right)+\varepsilon_{2 m}\left\langle c_{i}\left|\hat{\varepsilon}_{1} \cdot \vec{p}\right| v_{j}\right\rangle J_{p, n}\left(k_{2}\right)\right|^{2} \\
& \text { (discrete), } \\
& \alpha^{(2)}=A\left(1-\frac{1}{n^{2}}\right) \\
& x \sum_{i j m} \mid \varepsilon_{1 m}\left\langle c_{i}\left|\hat{\varepsilon}_{2} \cdot \vec{p}\right| v_{j}\right\rangle J_{p, n}\left(K_{1}\right)+\varepsilon_{2 m}\left\langle c_{i}\left|\hat{\varepsilon}_{1} \cdot \vec{p}\right| v_{j}\right\rangle J_{p, n}\left(K_{2}\right)^{2} \\
& \text { (overlap), } \\
& \alpha^{(2)}=A\left(1-\frac{1}{\eta^{2}}\right)\left(1-e^{-2 \pi \eta}\right)^{-1} \\
& x \sum_{i j m} \mid \varepsilon_{1 m}\left\langle c_{i}\left|\hat{\varepsilon}_{2} \cdot \vec{p}\right| v_{j}\right\rangle J_{p, k}\left(k_{1}\right)+\varepsilon_{2 m}\left\langle c_{i}\left|\hat{\varepsilon}_{1} \cdot \vec{p}\right| v_{j}\right\rangle J_{p, k}\left(\left.x_{2}\right|^{2}\right. \\
& \text { (continuum), } \\
& \text { where } A \equiv \frac{4 \pi}{3}\left(\frac{e}{m}\right)^{4} \frac{\hbar^{3}}{E_{B}^{3} a^{5} c^{2}} \frac{F_{2}}{n_{2} \sqrt{n_{1}} \omega_{1} \omega_{2}} \text {. We have } \\
& \text { changed the notation for the dielectric constants } n_{1} \text { and } \\
& n_{2} \text { to avoid confusion. } \\
& \text { In crystals of symmetry } T_{h} \text { or } O_{h}, i \cdot e \cdot \text { crystals } \\
& \text { in which } p_{x}, p_{y} \text {, and } p_{z} \text { transform according to different } \\
& \text { rows of the same representation, the dependence of the } \\
& \text { absorption coefficient on } \hat{\varepsilon}_{1} \text { and } \hat{\varepsilon}_{2} \text { is independent of the } \\
& \text { symmetries of }\left|c_{i}\right\rangle \text { and }\left|v_{j}\right\rangle \text {, as long as the matrix }
\end{aligned}
$$


elements are non-zero. To show this, we let $\left|c_{i}\right\rangle$ and $\left|v_{j}\right\rangle$ transform according to rows $i$ and $j$ of representations $\Gamma^{c}$ and $\Gamma^{v}$, respectively, let $p_{m}(m=x, y, z)$ transform according to row $s$ of $\Gamma^{4,5}$ of $O_{h}$ or $T_{h}$, and take the absolute square in Eq.4-6. The first term is proportional to

$$
\sum_{i j m} \varepsilon_{1 m}^{2}\left|\left\langle c_{i}\left|\hat{\varepsilon}_{2} \cdot \vec{p}\right| v_{j}\right\rangle\right|^{2}=\sum_{i j}\left|\left\langle c_{i}\left|\hat{\varepsilon}_{2} \cdot \vec{p}\right| v_{j}\right\rangle\right|^{2} .
$$

Now note that $\left\langle c_{i}\left|\hat{\varepsilon}_{2} \cdot \vec{p}\right| v_{j}\right\rangle=\left\langle c_{i}, v_{j}\left|\hat{\varepsilon}_{2} \cdot \vec{p}\right| g\right\rangle$, where $|g\rangle$ transforns under $\Gamma^{\prime}$. Since

$$
\left|c_{i}, v_{j}\right\rangle=\sum_{\mu \tau_{\mu} s}\left[\begin{array}{c}
\mu \tau_{\mu}, c v \\
s, i j
\end{array}\right]\left|\mu \tau_{\mu} s\right\rangle
$$

we have

$$
\begin{aligned}
& \sum_{i j}\left|\left\langle c_{i}\left|\hat{\varepsilon}_{2} \cdot \vec{p}\right| v_{j}\right\rangle\right|^{2}=\sum_{i j} \sum_{\mu \tau_{\mu} s} \sum_{\mu^{\prime} \tau_{\mu=}^{\prime} \varepsilon^{\prime}}\left[\begin{array}{r}
\mu \tau_{\mu}, c v \\
s, i j
\end{array}\right]^{*}\left[\begin{array}{r}
A^{\prime} \tau_{\mu}^{\prime}, c v \\
s^{\prime}, i j
\end{array}\right] \\
& X\left\langle\mu \tau_{\mu} s\left|\hat{\varepsilon}_{2} \cdot \vec{p}\right| g\right\rangle\left\langle g\left|\hat{\varepsilon}_{2} \cdot \vec{p}\right| \mu^{\prime} \tau_{\mu}^{\prime} s^{\prime}\right\rangle \\
& =\sum_{\tau_{4} \tau_{4}^{\prime} s s^{\prime}} \varepsilon_{2 s} \varepsilon_{2 s^{\prime}} \sum_{i j}\left[\begin{array}{r}
4 \tau_{4}, c v \\
s, i j
\end{array}\right]^{*}\left[\begin{array}{r}
4 \tau_{4}^{\prime}, c v \\
s^{\prime}, i j
\end{array}\right] \\
& x\left\langle 4 \tau_{4} s\left|p_{s}\right| g\right\rangle\left\langle g\left|p_{s^{\prime}}\right| 4 \tau_{4}^{\prime} s^{\prime}\right\rangle \\
& =\hat{\varepsilon}_{2} \cdot \hat{\varepsilon}_{2}\left|\langle p\rangle_{c v}\right|^{2}=\left|\langle p\rangle_{c v}\right|^{2},
\end{aligned}
$$

where

$$
\left|\langle p\rangle_{c v}\right|^{2} \equiv \sum_{\tau_{\psi}}\left|\sum_{i j}\left[\begin{array}{cc}
4 \tau_{\psi}, & c v \\
x, & i j
\end{array}\right]\left\langle c_{i}\left|p_{x}\right| v_{j}\right\rangle\right|^{2} \cdot(4-7)
$$

Using identical arguments for the other terms, the twophoton absorption coefficient becomes 


$$
\begin{aligned}
\alpha^{(2)} & =B\left\{\left|J_{1}\right|^{2}+\left|J_{2}\right|^{2}+\left(\hat{\varepsilon}_{1} \cdot \hat{\varepsilon}_{2}\right)^{2}\left[J_{1} J_{2}^{*}+J_{2} J_{1}^{*}\right]\right\} \\
& =B\left\{\left(\hat{\varepsilon}_{1} \times \hat{\varepsilon}_{2}\right)^{2}\left[\left|J_{1}\right|^{2}+\left|J_{2}\right|^{2}\right]+\left(\hat{\varepsilon}_{1} \cdot \hat{\varepsilon}_{2}\right)^{2}\left|J_{1}+J_{2}\right|^{2}\right\}
\end{aligned}
$$

where $J_{i}$ is $J_{p, n}\left(K_{i}\right)$ or $J_{p, k}\left(K_{i}\right)$, and

$$
B=A \times \begin{cases}\left(1-\frac{1}{n^{2}}\right) \frac{2 E_{B}}{n^{3}} \rho\left(E_{f}\right)\left|\langle p\rangle_{c v}\right|^{2} & \text { (discrete) } \\ \left(1-\frac{1}{n^{2}}\right)\left|\langle p\rangle_{c v}\right|^{2} & \text { (overlap) } \\ \left(1-\frac{1}{\eta^{2}}\right)\left(1-e^{-2 \pi \eta}\right)^{-1}\left|\langle p\rangle_{c V}\right|^{2} & \text { (continuum) }\end{cases}
$$

There are two significant properties of these results. The first is that the angular dependence of the absorption does not depend, in cubic and tetrahedral crystals, on the symmetries of the valence and conduction bands. The second is that it does not depend on the orientations of the polarization vectors with respect to the crystal axes, but only with respect to each other. Absorption for parallel polarizations is larger than for perpendicular polarizations, the ratio of their magnitudes being

$$
\frac{\alpha_{11}^{(2)}}{\alpha_{\perp}^{(2)}} \frac{\left|J_{1}+J_{2}\right|^{2}}{\left|J_{1}\right|^{2}+\left|J_{2}\right|^{2}}
$$

This ratio mast have a value between $I$ and 2 . Probably the largest error in this calculation is 
the restriction to the two band model, and it becomes important when, for example, a higher conduction band is close in energy to the conduction band of interest at the extremum. An example of this can be seen in the energy bands of CsI shown in Fig.5-8 of Chapter V, where the $\Gamma_{6}^{+}\left(A_{1 g}\right)$ and $\Gamma_{8}^{+}\left(E_{g}\right)$ conduction bands are close together. The. proximity of another conduction band has two principal effects: it necessitates keeping another term in the sum over intermediate states in Eq.2-23, and it can severely warp the energy bands near the extremum, invalidating the spherical band approximation. These effects immensely complicate calculations for the angular dependence. A second inadequacy of this calculation should be noted; that is, that although calculations have been made for discrete and continuum states, the calculations for the lowest energy discrete states are dubious, since these are the least weIl described by the Wannier model, especially for s-states, which enter the sum over intermediate states. This is particularly true in large bandgap insulators 54,55 such as the alkali halides. On the other hand, discrete p-states have not been observed experimentally in two-photon absorption, and, except for some error due to the sum over intermediate s-states, the calculations should be adequate for large n overlapping states and continuum states, insofar as the two spherical band model is adequate. 
B. Band Model

We will calculate the absorption coefficient for forbidden two-photon transitions ignoring the electron-hole interaction. We again assume a two band model in which the bands $\left|c_{i}(k)\right\rangle$ and $\left|v_{j}(k)\right\rangle$ are spherical near $\vec{k}=0$, and in which $\left|c_{i}(k)\right\rangle$ remain degenerate near $\vec{k}=0$, as do, $\left|V_{j}(k)\right\rangle$. Thus in Eq.2-10 we retain only the bands contained in the sets $\left\{\left|c_{i}\right\rangle\right\}$ and $\left\{\left|v_{j}\right\rangle\right\}$ in the intermediate states $n$, but we will keep all terms in the sum over $l$. Noting that $E_{c}(k)-E_{v}(k)=\hbar \omega_{1}+\hbar \omega_{2}, E q \cdot 2-10$ becomes

$$
\begin{aligned}
& P_{f g}^{(2)}= \\
& \frac{\hbar}{m} \sum_{l \neq c} \sum_{c_{i^{\prime}}}\left[\frac{\left\langle c_{i}|\vec{k} \cdot \vec{p}| l\right\rangle\left\langle\ell\left|\hat{\mathcal{E}}_{1} \cdot \vec{p}\right| c_{i^{\prime}}\right\rangle}{E_{c}-E_{l}}\right. \\
& \left.+\frac{\left\langle c_{i}\left|\hat{\varepsilon}_{1} \cdot \vec{p}\right| \ell\right\rangle\left\langle l|\vec{k} \cdot \vec{p}| c_{i^{\prime}}\right\rangle}{E_{c}-E_{l}}\right] \frac{\left\langle c_{i^{\prime}}\left|\hat{\varepsilon}_{2} \cdot \vec{p}\right| V_{j}\right\rangle}{\hbar \omega_{i}} \\
& -\frac{\hbar}{m} \sum_{l \neq v} \sum_{v_{j^{\prime}}} \frac{\left\langle c_{i}\left|\hat{\varepsilon}_{1} \cdot \vec{p}\right| v_{j^{\prime}}\right\rangle}{\hbar \omega_{2}}\left[\frac{\left\langle v_{j^{\prime}}|\vec{k} \cdot \vec{p}| \ell\right\rangle\left\langle l\left|\hat{\varepsilon}_{2} \cdot \vec{p}\right| v_{j}\right\rangle}{E_{v}-E_{l}}\right. \\
& \left.+\frac{\left\langle V_{j^{\prime}}\left|\hat{\varepsilon}_{2} \cdot \vec{p}\right| l\right\rangle\left\langle l|\vec{k} \cdot \vec{p}| V_{j}\right\rangle}{E_{v}-E_{l}}\right] \\
& \text { + terms with subscripts } 1 \text { and } 2 \text { interchanged (4-10) }
\end{aligned}
$$

Note that under the conditions imposed on the bands (see Appendix A) 
93

$$
\begin{aligned}
& \frac{1}{m} \sum_{l \neq c}\left[\frac{\left\langle c_{i}|\vec{k} \cdot \vec{p}| l\right\rangle\left\langle l\left|\hat{\varepsilon}_{1} \cdot \vec{p}\right| c_{i^{\prime}}\right\rangle}{E_{c}-E_{l}}\right. \\
& \left.+\frac{\left\langle c_{i}\left|\hat{\varepsilon}_{i} \cdot \vec{p}\right| l\right\rangle\left\langle\ell|\vec{k} \cdot \vec{p}| c_{i^{\prime}}\right\rangle}{E_{c}-E_{l}}\right] \\
& =\delta_{i i^{\prime}} \vec{k} \cdot\left[\left(\frac{\overleftrightarrow{m}}{m_{c}^{*}}\right)-\mathbb{Z}\right] \cdot \hat{\varepsilon}_{1}
\end{aligned}
$$

and

$$
\begin{aligned}
& \frac{1}{m} \sum_{l \neq v}\left[\frac{\left\langle v_{j \prime}|\vec{k} \cdot \vec{p}| \ell\right\rangle\left\langle\ell\left|\hat{\varepsilon}_{2} \cdot \vec{p}\right| v_{j}\right\rangle}{E_{v}-E_{l}}\right. \\
& \left.\quad+\frac{\left\langle v_{j^{\prime}}\left|\hat{\varepsilon}_{2} \cdot \vec{p}\right| l\right\rangle\left\langle\ell|\vec{k} \cdot \vec{p}| v_{j}\right\rangle}{E_{v}-E_{l}}\right] \\
& =\delta_{j j^{\prime}} \vec{k} \cdot\left[\left(\frac{m}{m_{v}^{*}}\right)-1\right] \cdot \hat{\varepsilon}_{2}
\end{aligned}
$$

where each effective mass tensor is a constant times a unit tensor. Eq .4-10 reduces to

$$
\begin{aligned}
& P_{f g}^{(2)}= \\
& \hbar \vec{k} \cdot\left[\frac{\vec{m}}{m_{c}^{*}}-1\right] \cdot \hat{\varepsilon}_{1} \frac{\left\langle c_{i}\left|\hat{\varepsilon}_{2} \cdot \vec{p}\right| v_{j}\right\rangle}{\hbar \omega_{1}} \\
& -\hbar \vec{k} \cdot\left[\frac{\vec{m}}{m_{v}^{*}}-1\right] \cdot \hat{\varepsilon}_{2} \frac{\left\langle c_{i}\left|\hat{\varepsilon}_{i} \cdot \vec{p}\right| v_{j}\right\rangle}{\hbar \omega_{2}} \\
& +\hbar \vec{k} \cdot\left[\frac{\vec{m}}{m_{c}^{*}}-\mathbb{1}\right] \cdot \hat{\varepsilon}_{2} \frac{\left\langle c_{i}\left|\hat{\varepsilon}_{1} \cdot \vec{p}\right| v_{j}\right\rangle}{\hbar \omega_{2}} \\
& -\hbar \vec{k} \cdot\left[\frac{\vec{m}}{m_{v}^{*}}-\mathbb{1}\right] \cdot \hat{\varepsilon}_{1} \frac{\left\langle c_{i}\left|\hat{\varepsilon}_{2} \cdot \vec{p}\right| v_{j}\right\rangle}{\hbar \omega_{1}}
\end{aligned}
$$




$$
\begin{aligned}
& =\hbar \vec{k} \cdot\left(\frac{\vec{m}}{\mu}\right) \cdot \hat{\varepsilon}_{1} \frac{\left\langle c_{i}\left|\hat{\varepsilon}_{2} \cdot \vec{p}\right| v_{j}\right\rangle}{\hbar \omega_{1}}+\hbar \vec{k} \cdot\left(\frac{\vec{m}}{\mu}\right) \cdot \hat{\varepsilon}_{2} \frac{\left\langle c_{i}\left|\hat{\varepsilon}_{1} \cdot \vec{p}\right| v_{j}\right\rangle}{\hbar \omega_{2}} \\
& =\hbar \frac{m}{\mu}\left[\vec{k} \cdot \hat{\varepsilon}_{1} \frac{\left\langle c_{i}\left|\hat{\varepsilon}_{2} \cdot \vec{p}\right| v_{j}\right\rangle}{\hbar \omega_{1}}+\vec{k} \cdot \hat{\varepsilon}_{2} \frac{\left\langle c_{i}\left|\hat{\varepsilon}_{1} \cdot \vec{p}\right| v_{j}\right\rangle}{\hbar \omega_{2}}\right] .
\end{aligned}
$$

Inserting this into Eq.2-7 we find

$$
\begin{aligned}
\alpha^{(2)=} & A^{\prime} \sum_{f}\left|P_{f g}^{(2)}\right|^{2} \delta\left[E_{f g}-\hbar\left(\omega_{1}+\omega_{2}\right)\right] \\
= & A^{\prime} \hbar^{2}\left(\frac{m}{\mu}\right)^{2} \sum_{i j} \frac{v}{(2 \pi)^{3}} \int d^{3} k \delta\left[E_{c}(k)-E_{v}(k)-\hbar\left(\omega_{1}+\omega_{2}\right\rangle\right] \\
& \quad \times\left|\vec{k} \cdot \hat{\varepsilon}_{1} \frac{\left\langle c_{i}\left|\hat{\varepsilon}_{2} \cdot \vec{p}\right| v_{j}\right\rangle}{\hbar \omega_{1}}+\vec{k}_{\cdot} \hat{\varepsilon}_{2} \frac{\left\langle c_{i}\left|\hat{\varepsilon}_{1} \cdot \vec{p}\right| v_{j}\right\rangle}{\hbar \omega_{2}}\right|^{2}
\end{aligned}
$$

where

$$
A^{\prime} \equiv \frac{(2 \pi)^{3}}{V} \frac{\hbar}{c^{2}}\left(\frac{e}{m}\right)^{4} \frac{F_{2}}{\kappa_{2} \sqrt{\pi_{1}} \omega_{1} \omega_{2}}
$$

As in the last section the form of

$$
\sum_{i j}\left|\vec{k} \cdot \hat{\varepsilon}_{1} \frac{\left\langle c_{i}\left|\hat{\varepsilon}_{2} \cdot \vec{p}\right| v_{j}\right\rangle}{\hbar \omega_{1}}+\vec{k} \cdot \hat{\varepsilon}_{2} \frac{\left\langle c_{i}\left|\hat{\varepsilon}_{1} \cdot \vec{p}\right| v_{j}\right\rangle}{\hbar \omega_{2}}\right|^{2}
$$

is independent of the symmetries of $c_{i}$ and $v_{j}$, provided that the matrix elements are non-zero; $i$. e. provided that $\Gamma^{c} x \Gamma^{v}$ contains $\Gamma^{4}$, so that we can write the preceding expression as

$$
k^{2} \sum_{s}\left[\hat{k} \cdot \hat{\varepsilon}_{1} \frac{\varepsilon_{2 s}}{\hbar \omega_{1}}+\hat{k} \cdot \hat{\varepsilon}_{2} \frac{\varepsilon_{1 s}}{\hbar \omega_{2}}\right]^{2}\left|\langle p\rangle_{c v}\right|^{2},
$$

where $\left|\langle p\rangle_{c v}\right|^{2}$ is defined by Eq.4-7. Near $\vec{k}=0$ let $E_{c}(k)-E_{r}(k)=E_{\text {gap }}+\frac{\hbar^{2} k^{2}}{2 \mu}$, and $d^{3} k=k^{2} d k d \Omega_{k}$. The Eq.4-II 
becomes

$$
\begin{aligned}
& \alpha(2)= \\
& A^{\prime} \hbar^{2}\left(\frac{m}{\mu}\right)^{2} \frac{V}{(2 \pi)^{3}}\left|\langle p\rangle_{c v}\right|^{2} \int k^{4} d k \delta\left[E_{g a p}+\frac{\hbar^{2} k^{2}}{2 \mu}-\hbar\left(\omega_{1}+\omega_{2}\right)\right] \\
& \quad \times \sum_{s} \int d \Omega_{k}\left[\hat{k} \cdot \hat{\varepsilon}_{1} \frac{\varepsilon_{2 s}}{\hbar \omega_{1}}+\hat{k} \cdot \hat{\varepsilon}_{2} \frac{\varepsilon_{1 s}}{\hbar \omega_{2}}\right]^{2}
\end{aligned}
$$

Let $z \equiv \frac{\hbar^{2} k^{2}}{2 \mu}$. Then

$$
\begin{aligned}
& \int k^{4} d k \delta\left[E_{\text {gap }}+\frac{\hbar^{2} k^{2}}{2 \mu}-\hbar\left(\omega_{1}+\omega_{2}\right)\right] \\
= & \frac{1}{2}\left(\frac{2 \mu}{\hbar^{2}}\right)^{5 / 2} \int z^{3 / 2} d z \delta\left[E_{\text {gap }}+z-\hbar\left(\omega_{1}+\omega_{2}\right)\right] \\
= & \frac{1}{2}\left(\frac{2 \mu}{\hbar^{2}}\right)^{5 / 2}\left[\hbar\left(\omega_{1}+\omega_{2}\right)-E_{\text {gap }}\right]^{3 / 2}
\end{aligned}
$$

This gives the well-known energy dependence for forbidden band-to-band transitions. Let $\left(K_{x}, K_{y}, K_{z}\right) \equiv \hat{k}$. Expanding the square in Eq.4-13 and using the integral

$$
\int K_{i} K_{j} d \Omega_{k}=\frac{4 \pi}{3} \delta_{i j}
$$

the two-photon absorption coefficient reduces to

$$
\begin{aligned}
& \alpha^{(2)}= \\
& \sqrt{\mu} B^{\prime}\left\{\left(\hat{\varepsilon}_{1} \times \hat{\varepsilon}_{2}\right)^{2}\left|\left(\frac{1}{\hbar \omega_{1}}\right)^{2}+\left(\frac{1}{\hbar \omega_{2}}\right)^{2}\right|+\left(\hat{\varepsilon}_{1} \cdot \hat{\varepsilon}_{2}\right)^{2}\left[\frac{1}{\hbar \omega_{1}}+\frac{1}{\hbar \omega_{2}}\right]^{2}\right\},
\end{aligned}
$$

where 


$$
B^{\prime} \equiv \frac{4 \pi}{3} \frac{e^{4}}{\hbar^{2} c^{2} m^{2}} \frac{F_{2} 2 \sqrt{2}}{k_{2} \sqrt{k_{1}} \omega_{1} \omega_{2}}\left[\hbar\left(\omega_{1}+\omega_{2}\right)-E_{g a p}\right]^{3 / 2}\left|\langle p\rangle_{c v}\right|^{2}
$$

The angular dependence of Eq.4-15 is seen to be identical in form with Eq.4-8, with $J_{i}$ replaced by $1 / \hbar \omega_{i}$. The ratio of parallel polarization absorption to perpendicular polarization absorption is

$$
\frac{\alpha_{11}^{(2)}}{\alpha_{\perp}^{(2)}}=\frac{\left[\frac{1}{\hbar \omega_{1}}+\frac{1}{\hbar \omega_{2}}\right]^{2}}{\left(\frac{1}{\hbar \omega_{1}}\right)^{2}+\left(\frac{1}{\hbar \omega_{2}}\right)^{2}}
$$

similar to Eq.4-9. Eq.4-17 is, of course, much simpler to evaluate than Eq.4-9, in view of the fact that the evaluation of $J_{i}$ requires a computer? The only experimental data available (which will be discussed in Chapter V) with which a comparison of Eq.4-17 and Eq.4-9 can be made, however, indicates that Eq.4-17 more accurately describes the polarization dependence than Fq.4-9. This could be due to uncertain values for the band gap and the exciton binding energy, both of which enter into the expression for $J_{i}$ in Mahan's model. A possible error also arises in the sum over intermediate s-states, although this is probably small for continuum final states. However with a single comparison the closer agreement of the simpler band model could simply be fortuitous in this case; future experiments should permit further comparison. 
C. p-like Valence Bands

An assumption in the two previous calculations was that the valence bands $\left\{\left|c_{i}(k)\right\rangle\right\}$ were degenerate near $\vec{k}=0$. This, of course, is never really the case; in general, if there is degeneracy at $\vec{k}=0$, then a short distance away in a general direction at least some of the degeneracy will be lifted. 56 In this section we will examine the splitting of $p$-like states near $k=0$ in a crystal with $O_{h}$ symmetry and determine what effect this has on the two-photon absorption coefficient for forbidden transitions.

Let the p-like valence band states at $\vec{k}=0$ be labeled $|x\rangle,|y\rangle$ and $|z\rangle$. In order to find the energies of the bands at a nearby $\vec{k}$ we must diagonalize the matrix ( see Appendix A)

$$
\left[\sum_{l \neq x, y, z} \frac{\langle i|\vec{k} \cdot \vec{p}| \ell\rangle\langle l|\vec{k} \cdot \vec{p}| j\rangle}{E_{i}-E_{l}}\right],
$$

where $i$ and $j$ are $x, y$ or $z$, and where all states and energies are at $\vec{k}=0$. This really requires knowledge of matrix elements involving all intermediate states, but substantial information can be obtained from symmetry considerations. Let us consider the intermediate states of the five possible symmetries $\Gamma^{\mu}\left(A_{i g}, A_{2 g}, E_{g}, T_{i g}\right.$ and $T_{2 g}$ ) one at a time. (The odd parity (ungerade) intermediate states do not contribute, since $p$ and $|i\rangle$ are both odd.) Thus we relabel the intermediate states: 


$$
\begin{aligned}
\sum_{l} \frac{|l\rangle\langle l|}{E_{i}-E_{l}}= & \sum \frac{\left|A_{1 g ; \alpha}\right\rangle\left\langle A_{1 g j \alpha}\right|}{E_{i}-E_{\alpha}}+\sum_{\alpha} \frac{\left|A_{2 g ; \alpha}\right\rangle\left\langle A_{2 g ; \alpha}\right|}{E_{i}-E_{\alpha}} \\
& \sum_{\alpha, m} \frac{\left|E_{g} m ; \alpha\right\rangle\left\langle E_{g} m ; \alpha\right|}{E_{i}-E_{\alpha}}+\sum_{\alpha, m} \frac{\left|T_{1 g} m ; \alpha\right\rangle\left\langle T_{i g} m \alpha\right|}{E_{i}-E_{\alpha}} \\
& \sum_{\alpha, m} \frac{\left|\tau_{2 g} m ; \alpha\right\rangle\left\langle T_{2 g} m ; \alpha\right|}{E_{i}-E_{\alpha}},
\end{aligned}
$$

where $m$ labels the row of the representation and $\alpha$ represents all other quantum numbers. The procedure for finding the form of the matrix is as follows: a) determine the matrices $\left[\left\langle i|\vec{k} \cdot \vec{p}| \Gamma^{\mu} m ; \alpha\right\rangle\right]$ for each representation $\Gamma^{\mu}$, b) multiply from the right by its adjoint, c) divide by $E_{i}-E_{\alpha}$ and sum over $\alpha$, and d) sum over representations $\Gamma^{\mu}$

a) First write $\left[\left\langle i|\vec{k} \cdot \vec{p}| \Gamma^{\mu} m ; \alpha\right\rangle\right]=\sum_{s} k_{s}\left[\left\langle i\left|p_{s}\right| \Gamma^{\mu} m ; \alpha\right\rangle\right]$ Each matrix $\left[\left\langle i\left|p_{s}\right| \Gamma^{\mu} m ; \alpha\right\rangle\right]$ is given in terms of an unknown constant $C_{\alpha}^{\mu}$ in the book by Koster et $a .{ }^{42}$ We have

$$
\begin{aligned}
& {\left[\left\langle i|\vec{k} \cdot \vec{p}| A_{1 g} ; \alpha\right\rangle\right]=\frac{1}{\sqrt{3}} C_{\alpha}^{1}\left[\begin{array}{l}
k_{x} \\
k_{y} \\
k_{z}
\end{array}\right]} \\
& {\left[\left\langle i|\vec{k} \cdot \vec{p}| A_{2 g} ; \alpha\right\rangle\right]=0}
\end{aligned}
$$


99

$$
\begin{aligned}
& {\left[\left\langle i|\vec{k} \cdot \vec{p}| E_{g} m ; \alpha\right\rangle\right]=C_{\alpha}^{3}\left[\begin{array}{cc}
-\frac{1}{\sqrt{6}} k_{x} & \frac{1}{\sqrt{2}} k_{x} \\
-\frac{1}{\sqrt{6}} k_{y} & -\frac{1}{\sqrt{2}} k_{y} \\
\sqrt{\frac{2}{3}} k_{z} & 0
\end{array}\right]} \\
& {\left[\left\langle i|\vec{k} \cdot \vec{p}| T_{1 g} ; \alpha\right\rangle\right]=C_{\alpha}^{4}\left[\begin{array}{ccc}
0 & -\frac{1}{\sqrt{2}} k_{z} & \frac{1}{\sqrt{2}} k_{y} \\
\frac{1}{\sqrt{2}} k_{z} & 0 & -\frac{1}{\sqrt{2}} k_{x} \\
-\frac{1}{\sqrt{2}} k_{y} & \frac{1}{\sqrt{2}} k_{x} & 0
\end{array}\right]} \\
& {\left[\left\langle i|\vec{k} \cdot \vec{p}| T_{2 g} m ; \alpha\right\rangle\right]=C_{\alpha}^{5}\left[\begin{array}{ccc}
0 & \frac{1}{\sqrt{2}} k_{z} & \frac{1}{\sqrt{2}} k_{y} \\
\frac{1}{\sqrt{2}} k_{z} & 0 & \frac{1}{\sqrt{2}} k_{x} \\
\frac{1}{\sqrt{2}} k_{y} & \frac{1}{\sqrt{2}} k_{x} & 0
\end{array}\right]}
\end{aligned}
$$

where the $C_{\alpha}^{k}$ are complex matrix elements. For example, $C_{\alpha}^{\prime}=\left\langle x\left|p_{x}\right| A_{i g} ; \alpha\right\rangle$.

$$
\begin{aligned}
& \text { b) }\left[\left\langle i|\vec{k} \cdot \vec{p}| A_{1 g} ; \alpha\right\rangle\left\langle A_{1 g} ; \alpha|\vec{k} \cdot \vec{p}| j\right\rangle\right]=\frac{\left|C_{\alpha}^{\prime}\right|^{2}}{3}\left[\begin{array}{ccc}
k_{x}^{2} & k_{x} k_{y} & k_{x} k_{z} \\
k_{x} k_{y} & k_{y}^{2} & k_{y} k_{z} \\
k_{x} k_{z} & k_{y} k_{z} & k_{z}^{2}
\end{array}\right]
\end{aligned}
$$

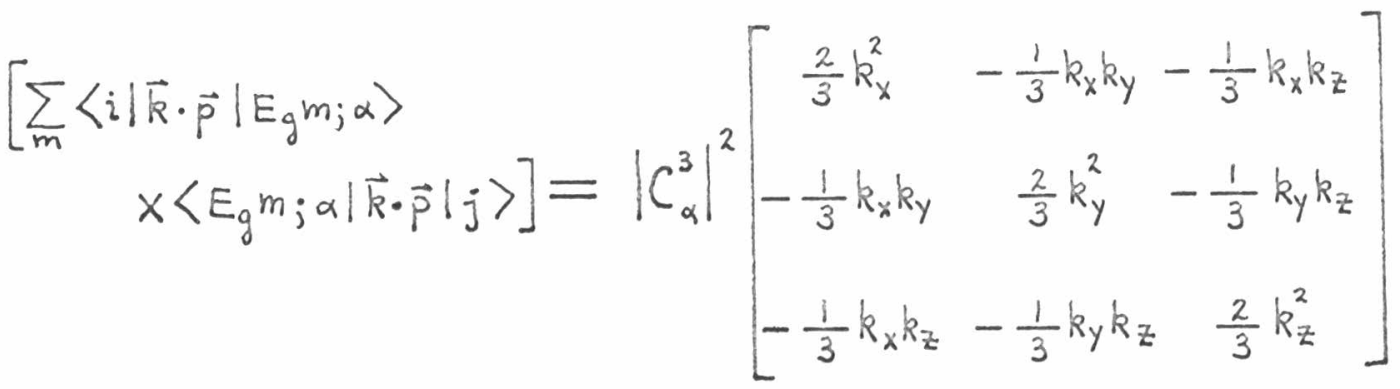




$$
\begin{aligned}
& \begin{array}{l}
{\left[\sum _ { m } \left\langlei|\vec{k} \cdot \vec{p}| T_{i g} m ; \alpha\right.\right.} \\
\left.x\left\langle T_{i g} m_{j} \alpha|\vec{k} \cdot \vec{p}| j\right\rangle\right]
\end{array}=\frac{\left|C_{\alpha}^{4}\right|^{2}}{2}\left[\begin{array}{ccc}
\left(k_{y}^{2}+k_{z}^{2}\right) & -k_{x} k_{y} & -k_{x} k_{z} \\
-k_{x} k_{y} & \left(k_{x}^{2}+k_{z}^{2}\right) & -k_{y} k_{z} \\
-k_{x} k_{z} & -k_{y} k_{z} & \left(k_{x}^{2}+k_{y}^{2}\right)
\end{array}\right] \\
& {\left[\begin{array}{l}
\sum_{m}\left\langle i|\vec{k} \cdot \vec{p}| T_{2 g} m ; \alpha\right\rangle \\
x\left\langle T_{2 g} m_{j} \alpha|\vec{k} \cdot \vec{p}| j\right\rangle
\end{array}\right]=\frac{\left|C_{\alpha}^{5}\right|^{2}}{2}\left[\begin{array}{ccc}
\left(k_{y}^{2}+k_{z}^{2}\right) & k_{x} k_{y} & k_{x} k_{z} \\
k_{x} k_{y} & \left(k_{x}^{2}+k_{z}^{2}\right) & k_{y} k_{z} \\
k_{x} k_{z} & k_{y} k_{z} & \left(k_{x}^{2}+k_{y}^{2}\right)
\end{array}\right]}
\end{aligned}
$$

c) For the sum over all sets $\alpha$ having symmetry $\Gamma^{\mu}$ we make the definition

$$
\sigma^{\mu} \equiv \sum_{\alpha} \frac{\left|C_{\alpha}^{\mu}\right|^{2}}{E_{i}-E_{\alpha}}
$$

where $\sigma^{\mu}$ is a real constant.

d) Summing over $\mu$, and keeping in mind that $k^{2}=k_{x}^{2}+k_{y}^{2}+k_{z}^{2}$, we obtain

$\left[\sum_{l \neq x, y, z} \frac{\langle i|\vec{k} \cdot \vec{p}| \ell\rangle\langle\ell|\vec{k} \cdot \vec{p}| j\rangle}{E_{i}-E_{l}}\right]$

$$
=\frac{1}{2}\left(\sigma^{4}+\sigma^{5}\right) k^{2} \mathbb{1}+\left[\begin{array}{ccc}
\xi k_{x}^{2} & \eta k_{x} k_{y} & \eta k_{x} k_{z} \\
\eta k_{x} k_{y} & \xi k_{y}^{2} & \eta k_{y} k_{z} \\
\eta k_{x} k_{z} & \eta k_{y} k_{z} & \xi k_{z}^{2}
\end{array}\right]
$$

where

$$
\begin{aligned}
& \xi \equiv \frac{1}{3} \sigma^{1}+\frac{2}{3} \sigma^{3}-\frac{1}{2} \sigma^{4}-\frac{1}{2} \sigma^{5} \\
& \eta \equiv \frac{1}{3} \sigma^{1}-\frac{1}{3} \sigma^{3}-\frac{1}{2} \sigma^{4}+\frac{1}{2} \sigma^{5} .
\end{aligned}
$$


Eq.4-19 is identical to that given by shockley 57 for a p-like valence band, except that, in addition, we have obtained the dependence of the coefficients $\xi$ and $\eta$ on the intermediate state symmetries, by Eq.4-20 and Eq.4-18. The diagonalization of Eq.4-19 involves the solution of a cubic secular equation, and the energies of the bands, given by

$$
\begin{aligned}
E_{s}(\vec{k}) & =E_{s}(0)+\frac{\hbar^{2} k^{2}}{2 m}+\frac{\hbar^{2}}{m^{2}} \sum_{l \neq\{s\}} \frac{\langle s|\vec{k} \cdot \vec{p}| l\rangle\langle l|\vec{k} \cdot \vec{p}| s\rangle}{E_{s}-E_{l}} \\
& =E_{s}(0)+\frac{\hbar^{2}}{2} \sum_{\beta} k_{\beta}^{2}\left[\frac{1}{\left.m_{s}^{*(n)}\right)}\right]_{\beta \beta,}
\end{aligned}
$$

depend, for a given magnitude $k$, on the direction $\hat{k}$. The states $|s\rangle \equiv|1\rangle,|2\rangle,|3\rangle$ are

$$
|s\rangle=\sum_{j=x, y, z} u_{j s}|j\rangle,
$$

where $U$ (a function of $\hat{k}$ ) is the unitary matrix which diagonalizes Eq.4-19.

Let us suppose that $\beta=\eta$. As will be shown in a moment this leads to spherical energy bands. Our supposition implies, by Eq.4-20, that $\sigma^{3}=\sigma^{5}$, or that, in the sum over intermediate states, those of symmetry $\mathrm{E}_{g}$ give the same contribution as those of symmetry $\mathbb{T}_{2 q} \cdot$ Usually the states with these symmetries of most consequence are those that derive from atomic d-states. We can therefore say, approximately, that p-like valence bands at 
$\vec{k}=0$ are spherical if the $E_{g}$ and $T_{2} g$ states derived from atomic d-states are negligibly crystal-field split. (This is also true of $\Gamma^{6}$ and $\Gamma^{8}$ bands derived from spin-orbit split $T_{1 g}$ bands (which will not be shown here) as can be seen by inspecting the valence bands of KI and CsI of Fig.5-9 and Fig.5-7.)

$$
\text { Returning to Eq.4-19, with } \rho=\eta \text {, let us focus }
$$

our attention on the second matrix on the right, since the first is invariant under any unitary transformation. W'e will factor out the magnitude $k$ and, as in the last section, let $\hat{k}=\left(k_{x}, k_{y}, k_{z}\right)$. The determinantal equation

$$
\operatorname{det}\left|\begin{array}{ccc}
k_{x}^{2}-\lambda & k_{x} k_{y} & k_{x} k_{z} \\
k_{x} k_{y} & k_{y}^{2}-\lambda & k_{y} k_{z} \\
k_{x} k_{z} & k_{y} k_{z} & k_{z}^{2}-\lambda
\end{array}\right|=0
$$

has solutions $\lambda=1$ and $\lambda=0$ (twice). The unitary transformation which gives the states $|S\rangle$ in terms of $|x\rangle,|y\rangle$ and $|z\rangle$ is obtained from

$$
\begin{aligned}
& \left(k_{x}^{2}-\lambda_{s}\right) u_{x s}+k_{x} k_{y} u_{y s}+k_{x} k_{z} u_{z s}=0 \\
& k_{x} k_{y} u_{x s}+\left(k_{y}^{2}-\lambda_{s}\right) u_{y s}+k_{y} k_{z} u_{z s}=0 \\
& k_{x} k_{z} u_{x s}+k_{y} k_{z} u_{y s}+\left(k_{z}^{2}-\lambda_{s}\right)=0
\end{aligned}
$$

For $\lambda_{s}=1 \equiv \lambda_{1}, \quad\left(u_{x_{1}}, u_{y_{1}}, u_{z_{1}}\right)=\left(k_{x}, k_{y}, k_{z}\right)$. Since $\lambda_{2}=\lambda_{3}=0$ we can choose any two vectors. orthogonal to $\left(K_{x}, K_{y}, K_{z}\right)$. We will choose these 
to be $\left(\theta_{x}, \theta_{y}, \theta_{z}\right)$ and $\left(\phi_{x}, \phi_{y}, \phi_{z}\right)$ which are the usual unit vectors denoted by $\hat{\theta}$ and $\hat{\phi}$ :

$$
\begin{aligned}
& \hat{k}=\hat{\imath} \sin \theta \cos \phi+\hat{\jmath} \sin \theta \sin \phi+\hat{k} \cos \theta \\
& \hat{\theta}=\hat{\imath} \cos \theta \cos \phi+\hat{\jmath} \cos \theta \sin \phi-\hat{k} \sin \theta \\
& \phi=-\hat{\imath} \sin \phi+\hat{\jmath} \cos \phi
\end{aligned}
$$

In summation we have

$$
\begin{aligned}
& {\left[\sum_{l}^{\prime} \frac{\langle s|\vec{k} \cdot \vec{p}| \ell\rangle\left\langle\ell|\vec{k} \cdot \vec{p}| s^{\prime}\right\rangle}{E_{s}-E_{l}}\right]} \\
& =\frac{1}{2}\left[\sigma^{4}+\sigma^{5}\right] k^{2} 1+\xi k^{2}\left[\begin{array}{lll}
1 & 0 & 0 \\
0 & 0 & 0 \\
0 & 0 & 0
\end{array}\right]
\end{aligned}
$$

with (see Appendix A)

$$
\begin{gathered}
{\left[\sigma^{4}+\sigma^{5}\right]+2 \xi=\left[\frac{m^{2}}{m_{1}^{*}}-m\right]} \\
{\left[\sigma^{4}+\sigma^{5}\right]=\left[\frac{m^{2}}{m_{2}^{*}}-m\right] \quad(4-21)} \\
\xi=\frac{1}{2}\left[\frac{m^{2}}{m_{1}^{*}}-\frac{m^{2}}{m_{2}^{*}}\right]=\frac{m^{2}}{2}\left[\frac{1}{\mu_{2}}-\frac{1}{\mu_{1}}\right] \equiv \frac{m^{2}}{2}\left(\Delta \frac{1}{\mu}\right) \\
\text { where } m_{1}^{*} \text { and } m_{2}^{*} \text { are the effective masses of the } \\
\text { non-degenerate and the two-fold degenerate bands, respec- } \\
\text { tively, and } \mu_{s}^{-1} \equiv\left(m_{c}^{*}\right)^{-1}-\left(m_{s}^{*}\right)^{-1} \text { is the reduced }
\end{gathered}
$$
reciprocal eprective mass.

$$
|s\rangle=\sum_{j} u_{j s}|j\rangle
$$


where

$$
u=\left[\begin{array}{lll}
K_{x} & \theta_{x} & \phi_{x} \\
K_{y} & \theta_{y} & \phi_{y} \\
K_{z} & \theta_{z} & \phi_{z}
\end{array}\right] .
$$

The problem now is to determine the angular dependence of absorption for transitions to a conduction band (which we will assume to be totally symmetric) from two spherical valence bands. The situation is depicted in Fig.4-1. The degeneracies of the bands are indicated in parentheses (the ordering of the valence bands is arbitrary). In calculating the absorption coefficient we will need to know the expression

$$
\sum_{l}^{\prime}\left\{\frac{\langle s|\vec{k} \cdot \vec{p}| l\rangle\left\langle l|\hat{\varepsilon} \cdot \vec{p}| s^{\prime}\right\rangle}{E_{s}-E_{l}}+\frac{\langle s|\hat{\varepsilon} \cdot \vec{p}| l\rangle\left\langle l|\vec{k} \cdot \vec{p}| s^{\prime}\right\rangle}{E_{s}-E_{l}}\right\} .
$$

In a manner identical to that of finding Eq.4-19 we obtain (with $\hat{\varepsilon} \equiv(l, m, n)$ )

$$
\begin{aligned}
& \sum_{l}^{\prime}\left\{\frac{\langle i|\vec{k} \cdot \vec{p}| l\rangle\langle 2|\hat{\varepsilon} \cdot \vec{p}| j\rangle}{E_{i}-E_{l}}+\frac{\langle i|\hat{\varepsilon} \cdot \vec{p}| l\rangle\langle l|\vec{k} \cdot \vec{p}| j\rangle}{E_{i}-E_{l}}\right\} \\
& =\left[\sigma^{4}+\sigma^{5}\right](\hat{\varepsilon} \cdot \vec{k}) \mathbb{1}+\xi\left[\begin{array}{ccc}
2 l k_{x} & 2 k_{y}+m k_{x} & l k_{z}+n k_{x} \\
l k_{y}+m k_{x} & 2 m k_{y} & m k_{z}+n k_{y} \\
2 k_{z}+n k_{x} & m k_{z}+n k_{y} & 2 n k_{z}
\end{array}\right]
\end{aligned}
$$

Multiplication from the left by $u^{+}$and from the right by 


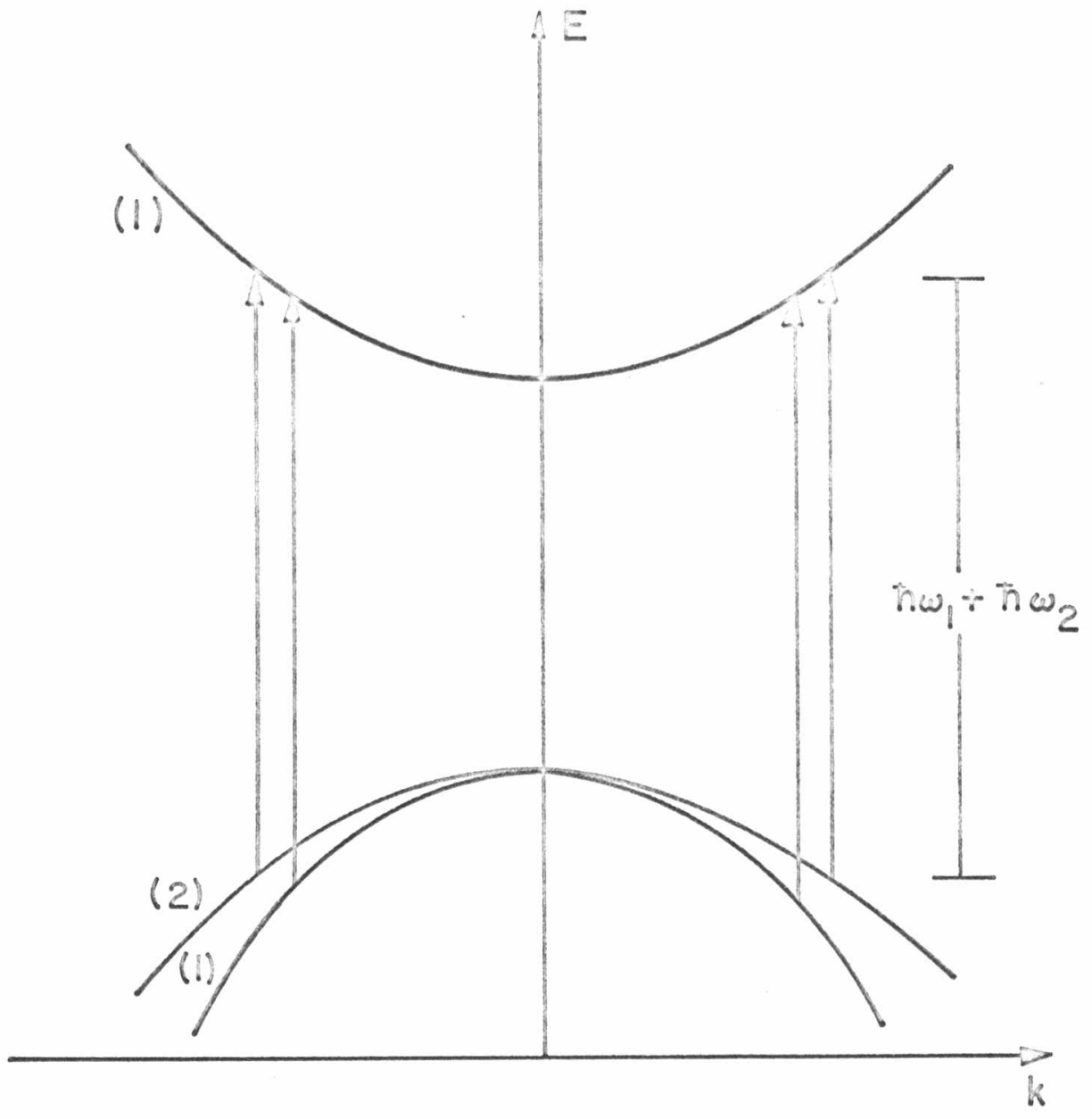

Fig.4-1. Two-photon absorption for spherecal p-like bands 
U yields

$$
\begin{array}{r}
\left.\sum_{l}\left\{\frac{\langle s|\vec{k} \cdot \vec{p}| l\rangle\left\langle l|\hat{\varepsilon} \cdot \vec{p}| s^{\prime}\right\rangle}{E_{s}-E_{l}}+\frac{\langle s|\hat{\varepsilon} \cdot \vec{p}| l\rangle\left\langle l|\vec{k} \cdot \vec{p}| s^{\prime}\right\rangle}{E_{s}-E_{l}}\right\}\right] \\
=\left[\sigma^{4}+\sigma^{5}\right](\hat{\varepsilon} \cdot \hat{k}) k \mathbb{1}+\xi k\left[\begin{array}{ccc}
2 \hat{\varepsilon} \cdot \hat{k} & \hat{\varepsilon} \cdot \hat{\theta} & \hat{\varepsilon} \cdot \hat{\phi} \\
\hat{\varepsilon} \cdot \hat{\theta} & 0 & 0 \\
\hat{\varepsilon} \cdot \hat{\phi} & 0 & 0
\end{array}\right]
\end{array}
$$

The second-order matrix element of Eq.2-10 is

$$
\begin{aligned}
& P_{f g}^{(2)}=\frac{\hbar k}{m} \sum_{l \neq c c}\left[\frac{\langle c|\hat{k} \cdot \vec{p}| \ell\rangle\left\langle l\left|\hat{\varepsilon}_{1} \cdot \vec{p}\right| c\right\rangle}{E_{c}-E_{l}}\right. \\
& \left.+\frac{\left\langle c\left|\hat{\varepsilon}_{1} \cdot \vec{p}\right| 2\right\rangle\langle 2|\hat{k} \cdot \vec{p}| c\rangle}{E_{c}-E_{l}}\right] \frac{\left\langle c\left|\hat{\varepsilon}_{1} \cdot \vec{p}\right| s^{\prime}\right\rangle}{\hbar \omega_{1}} \\
& -\frac{\hbar k}{m} \sum_{l \neq v} \sum_{s} \frac{\left\langle c\left|\hat{\varepsilon}_{1} \cdot \vec{p}\right| s\right\rangle}{\hbar \omega_{2}}\left[\frac{\langle s|\hat{k} \cdot \vec{p}| \ell\rangle\left\langle l\left|\hat{\varepsilon}_{2} \cdot \vec{p}\right| s^{\prime}\right\rangle}{E_{s^{\prime}}-E_{l}}\right. \\
& \left.+\frac{\left\langle s\left|\hat{\varepsilon}_{2} \cdot \vec{p}\right| \ell\right\rangle\left\langle 2|\hat{k} \cdot \vec{p}| s^{\prime}\right\rangle}{E_{s^{\prime}}-E_{l}}\right] \\
& +(1 \leftrightarrow 2) \\
& \equiv \frac{\hbar k}{m} P_{c s^{\prime}}^{(2)},
\end{aligned}
$$

where the symbol ( $1 \leftrightarrow 2$ ) neans similar terms with the subscripts 1 and 2 on $\hat{\varepsilon}$ and $\omega$ interchanged. Substitution into Eq.2-7 yields

$$
\alpha^{(2)}=A^{\prime} \sum_{f}\left|P_{f g}\right|^{2} \delta\left[E_{f g}-\hbar\left(\omega_{1}+\omega_{2}\right)\right]
$$




$$
\begin{aligned}
& =A^{\prime} \frac{\hbar^{2}}{m^{2}} \frac{v}{(2 \pi)^{3}} \sum_{s^{\prime}} \int d^{3} k k^{2}\left|P_{c s^{\prime}}^{(2)}\right|^{2} \delta\left[E_{g a p}+\frac{\hbar k^{2}}{2 \mu_{s^{\prime}}}-\hbar\left(\omega_{1}+\omega_{2}\right)\right] \\
& =A^{\prime} \frac{\hbar^{2}}{m^{2}} \frac{v}{(2 \pi)^{3}} \int k^{4} d k \sum_{s^{\prime}} \int d \Omega_{k}\left|P_{c s^{\prime}}\right|^{2} \delta\left[E_{g a p}+\frac{\hbar k^{2}}{2 \mu_{s^{\prime}}}-\hbar\left(\omega_{1}+\omega_{2}\right)\right]
\end{aligned}
$$

where $A^{\prime}$ is defined in $\mathrm{Eq} \cdot 4-12$. We perform the integral, over k, using Eq. 4-14:

$$
\alpha^{(2)}=A^{\prime} \frac{\hbar^{2}}{m^{2}} \frac{v}{(2 \pi)^{3}} \frac{1}{2}\left(\frac{2}{\hbar^{2}}\right)^{5 / 2}\left[\frac{1}{\hbar}\left(\omega_{1}+\omega_{2}\right)-E_{g a p}\right]^{3 / 2} \sum_{s^{\prime}} \int d \Omega_{k} \mu_{s^{\prime}}^{5 / 2}\left|P_{c s^{\prime}}^{(2)}\right|^{2}
$$

Using Eq.L-2L and the fact that $\langle c|\hat{c} \cdot \vec{p}| s\rangle=\hat{\varepsilon} \cdot \hat{\gamma}_{s}\left\langle c\left|p_{x}\right| x\right\rangle$ $\equiv \hat{\varepsilon} \cdot \hat{\gamma}_{s}\langle p\rangle_{c V}$, where $\hat{\gamma}_{s}$, by Fq.4-22 and Eq.4-23, is $\hat{k}, \hat{\theta}$ or $\hat{\phi}$, we obtain

$$
\begin{aligned}
& \alpha^{(2)}=A^{\prime} \frac{v}{(2 \pi)^{3}} \frac{\hbar^{2}}{m^{2}} \frac{1}{2}\left(\frac{2}{\hbar^{2}}\right)^{5 / 2}\left[\hbar\left(\omega_{1}+\omega_{2}\right)-E_{g a p}\right]^{3 / 2}\left|\langle p\rangle_{c v}\right|^{2} \int d \Omega_{k} \\
& x\left\{\mu_{1}^{5 / 2} \mid \frac{\left(\hat{\varepsilon}_{1} \cdot \hat{k}\right)\left(\hat{\varepsilon}_{2} \cdot \hat{k}\right)}{\hbar \omega_{1}}\left[\frac{m^{2}}{m_{c}^{* / 2}}-m\right]-\frac{(\hat{\varepsilon} \cdot \hat{k})\left(\hat{\varepsilon}_{2} \cdot \hat{k}\right)}{\hbar \omega_{2}}\left[\frac{m^{2}}{m_{1}^{* k^{*}}}-m\right]\right. \\
& -\left[\frac{\left(\hat{\varepsilon}_{1} \cdot \hat{\theta}\right)\left(\hat{\varepsilon}_{2} \cdot \hat{\theta}\right)}{\hbar \omega_{2}}+\frac{\left(\hat{\varepsilon}_{1} \cdot \hat{\phi}\right)\left(\hat{\varepsilon}_{2} \cdot \hat{\phi}\right)}{\hbar \omega_{2}}\right] \frac{1}{2}\left(\Delta \frac{1}{\mu}\right)+\left.(\mid \leftrightarrow 2)\right|^{2} \\
& +\mu_{2}^{5 / 2} \mid \frac{\left(\hat{\varepsilon}_{1} \cdot \hat{k}\right)\left(\hat{\varepsilon}_{2} \cdot \hat{\theta}\right)}{\hbar \omega_{1}}\left[\frac{m^{2}}{m_{c}^{*}}-m\right]-\frac{\left(\hat{\varepsilon}_{1} \cdot \hat{k}\right)\left(\hat{\varepsilon}_{2} \cdot \hat{\theta}\right)}{\hbar \omega_{2}} \frac{1}{2}\left(\Delta \frac{1}{\mu}\right) \\
& -\frac{\left(\hat{\varepsilon}_{1} \cdot \hat{\theta}\right)\left(\hat{\varepsilon}_{2} \cdot \hat{k}\right)}{\hbar \omega_{2}}\left[\frac{m^{2}}{m_{2}^{*}}-m\right]+\left.(1 \leftrightarrow 2)\right|^{2}
\end{aligned}
$$




$$
\begin{aligned}
+\mu_{2}^{5 / 2} \mid \frac{\left(\hat{\varepsilon}_{1} \cdot \hat{k}\right)\left(\hat{\varepsilon}_{2} \cdot \hat{\phi}\right)}{\hbar \omega_{1}}\left[\frac{m^{2}}{m_{c}^{*}}-m\right]-\frac{\left(\hat{\varepsilon}_{1} \cdot \hat{k}\right)\left(\hat{\varepsilon}_{2} \cdot \hat{k}\right)}{\hbar \omega_{2}} \frac{1}{2}\left(\Delta \frac{1}{\mu}\right) \\
\\
\left.-\frac{\left(\hat{\varepsilon}_{1} \cdot \hat{\dot{p}}\right)\left(\hat{\varepsilon}_{2} \cdot \hat{k}\right)}{\hbar \omega_{2}}\left[\frac{m^{2}}{m_{c}^{*}}-m\right]+\left.(1 \leftrightarrow 2)\right|^{2}\right\}
\end{aligned}
$$

The integrand can be rewritten

$$
\begin{aligned}
& m^{4} \mu_{1}^{5 / 2} \mid\left(\hat{\varepsilon}_{1} \cdot \hat{k}_{)}\right)\left(\hat{\varepsilon}_{2} \cdot \hat{k}\right)\left[\frac{1}{\hbar \omega_{1}}+\frac{1}{\hbar \omega_{2}}\right] \frac{1}{\mu_{1}}-\left.\left[\left(\hat{\varepsilon}_{1} \cdot \hat{\theta}\right)\left(\hat{\varepsilon}_{2} \cdot \hat{\theta}\right)+\left(\hat{\varepsilon}_{1} \cdot \hat{\phi}\right)\left(\hat{\varepsilon}_{2} \cdot \hat{\phi}\right)\right]\left[\frac{1}{\hbar \omega_{1}}+\frac{1}{\hbar \omega_{2}}\right] \frac{1}{2}\left(\Delta \frac{1}{\mu}\right)\right|^{2} \\
&+m^{4} \mu_{2}^{5 / 2} \mid\left(\hat{\varepsilon}_{1} \cdot \hat{k}\right)\left(\hat{\varepsilon}_{2} \cdot \hat{\theta}\right)\left[\frac{1}{\hbar \omega_{1}} \frac{1}{\mu_{2}}-\frac{1}{\hbar \omega_{2}} \frac{1}{2}\left(\Delta \frac{1}{\mu}\right)\right] \\
&+\left.\left(\hat{\varepsilon}_{1} \cdot \hat{\theta}\right)\left(\hat{\varepsilon}_{2} \cdot \hat{k}\right)\left[\frac{1}{\hbar \omega_{2}} \frac{1}{\mu_{2}}-\frac{1}{\hbar \omega_{1}} \frac{1}{2}\left(\Delta \frac{1}{\mu}\right)\right]\right|^{2} \\
&+m^{4} \mu_{2}^{5 / 2} \mid\left(\hat{\varepsilon}_{1} \cdot \hat{k}\right)\left(\hat{\varepsilon}_{2} \cdot \hat{\phi}\right)\left[\frac{1}{\hbar \omega_{1}} \frac{1}{\mu_{2}}-\frac{1}{\hbar \omega_{2}} \frac{1}{2}\left(\Delta \frac{1}{\mu}\right)\right] \\
& \quad+\left.\left(\hat{\varepsilon}_{1} \cdot \hat{\phi}\right)\left(\hat{\varepsilon}_{2} \cdot \hat{k}\right)\left[\frac{1}{\hbar \omega_{2}} \frac{1}{\mu_{2}}-\frac{1}{\hbar \omega_{1}} \frac{1}{2}\left(\Delta \frac{1}{\mu}\right)\right]\right|^{2} .
\end{aligned}
$$

Taking the absolute squares and integrating over the angles we obtain, after considerable algebra, 


$$
\begin{aligned}
& \alpha^{(2)=} \frac{1}{5} B^{\prime} \\
& \times\left[( \hat { \varepsilon } _ { 1 } \cdot \hat { \varepsilon } _ { 2 } ) ^ { 2 } \left\{\sqrt{\mu_{1}}\left[3-2\left(\frac{\mu_{1}}{\mu_{2}}-1\right)+2\left(\frac{\mu_{1}}{\mu_{2}}-1\right)^{2}\right]\right.\right. \\
& \left.+\frac{1}{2} \sqrt{\mu_{2}}\left[1+\frac{\mu_{2}}{\mu_{1}}\right]^{2}\right\}\left[\frac{1}{\hbar \omega_{1}}+\frac{1}{\hbar \omega_{2}}\right]^{2} \\
& +\left(\hat{\varepsilon}_{1} \times \hat{\varepsilon}_{2}\right)^{2}\left\{\frac{1}{4} \sqrt{\mu_{1}}\left[1+\frac{\mu_{1}}{\mu_{2}}\right]^{2}\left[\frac{1}{\hbar \omega_{1}}+\frac{1}{\hbar \omega_{2}}\right]^{2}\right. \\
& \\
& \left.\left.\quad+2 \sqrt{\mu_{2}}\left[2 a^{2}+2 b^{2}-a b\right]\right\}\right]
\end{aligned}
$$

where

$$
\begin{aligned}
& a \equiv \frac{1}{\hbar \omega_{1}}-\frac{1}{\hbar \omega_{2}} \frac{1}{2}\left(1-\frac{\mu_{2}}{\mu_{1}}\right), \\
& b \equiv \frac{1}{\hbar \omega_{2}}-\frac{1}{\hbar \omega_{1}} \frac{1}{2}\left(1-\frac{\mu_{2}}{\mu_{1}}\right),
\end{aligned}
$$

and $B^{\prime}$ is defined by $E Q_{1} \cdot 4-16$.

Thus we have the result, when the conduction band is totally symmetric, that a spherical splitting or a p-like valence band does not alter the absorption's independence of the orientation of the polarizations with respect to the crystal axes. The splitting, however, changes the expression for the ratio of parallel polarization absorption to perpendicular polarization absorption. This ratio is seen to be a rather complicated function of the photon energies and the ratio of reduced effective masses. To determine how severe the splitting effect is on this 
ratio, the maximum and minimum of $\alpha_{11}^{(2)} / \alpha_{\perp}^{(2)}$ has been plotted, and the curves are given in Fig.4-2 and Fig.4-3. Fig.4-2 applies when $\mu_{1} \geqslant \mu_{2}$ and Fig.4-3 is for $\mu_{2} \geq \mu_{1}$. The upper curve in each figure represents the maximum ratio, which occurs when $\hbar \omega_{1}=\hbar \omega_{2}$; the lower curves give the minimum ratio, which is the limit as $\hbar \omega_{1}$ becomes large compared with $\hbar \omega_{2}$, or vice-versa. Thus, for a given reduced mass ratio, the ratio of $\alpha_{11}^{(2)}$ to $\alpha_{\perp}^{(2)}$ lies within the shaded portion, its precise value determined by the photon energies. The range of $\alpha_{11}^{(2)} / \alpha_{1}^{(2)}$ does not differ too drastically from the range $1 \rightarrow 2$ when $\mu_{2}=\mu_{1}$ except when $\mu_{1}$ is much larger than $\mu_{2}$. The curves in Fig.4-2 approach the limit 8 with no photon energy dependence for large $\mu_{1} / \mu_{2}$.

For crystals in which two-photon forbidaen transitions at the $\Gamma$-point have been observed, mostly heavy halides, the spin-orbit coupling is appreciable. Although it is a simple matter to determine the energy dependence of spinorbit split $p$-like bands near $\vec{k}=0$, finding an equation analogous to Eq.4-24 is not, nor would be the evaluation of the required integrals over k-space. For this reason the inclusion of spin-orbit coupling has been omitted. In itself our calculation is of limited usefulness until experiments are performed on crystals having negligibly spin-orbit split p-like spherical valence bands, such as, perhaps, some chlorides. However, this calculation serves 
to show that $\vec{k} \cdot \vec{p}$ splitting does affect the polarization dependence, at the very least to the extent of altering the ratio of maximum to minimum absorption. 

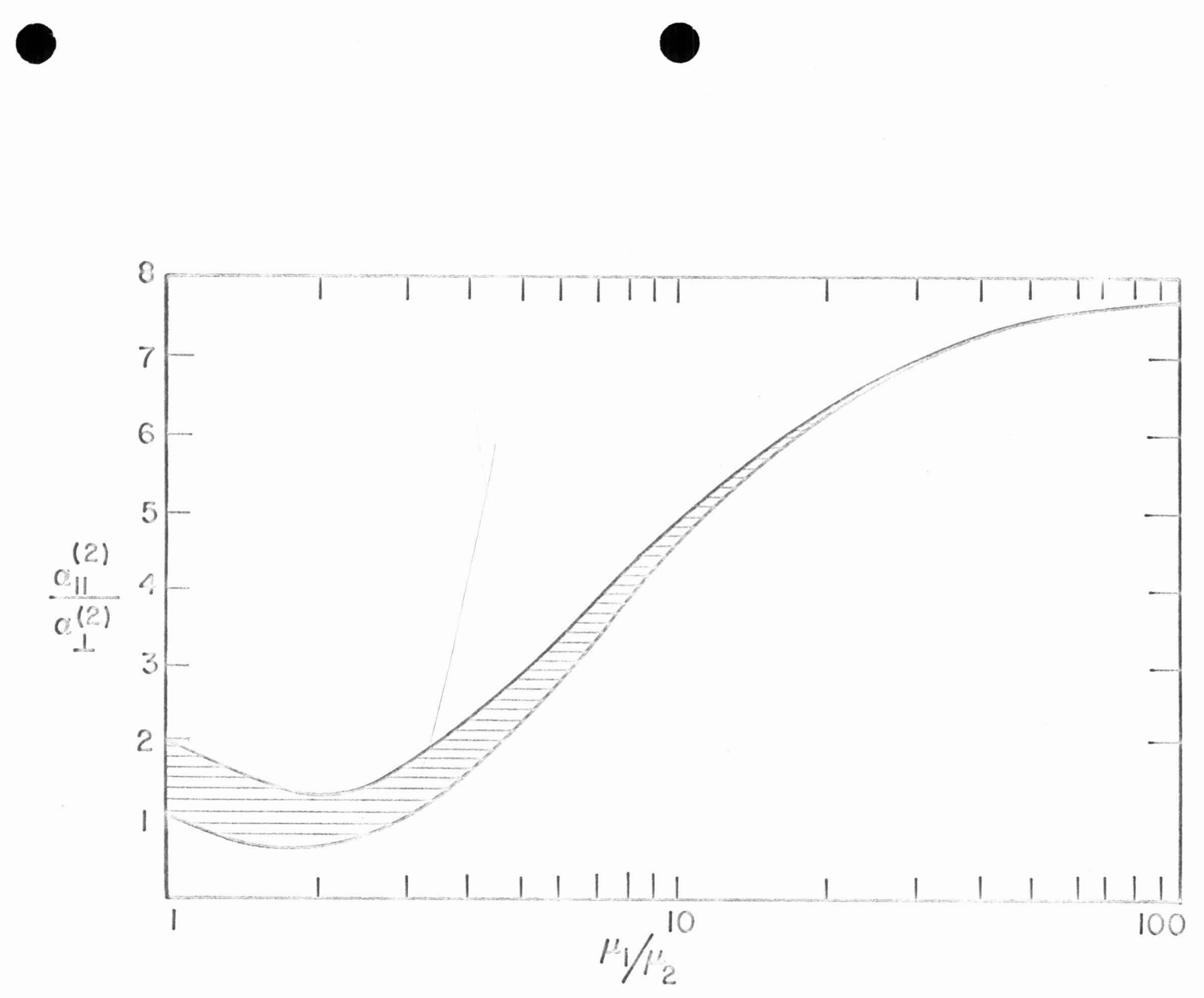

Fig.4-2. Ratio of parallel to perpendicular polarization absorption for $\mu_{1}>\mu_{2}$ 


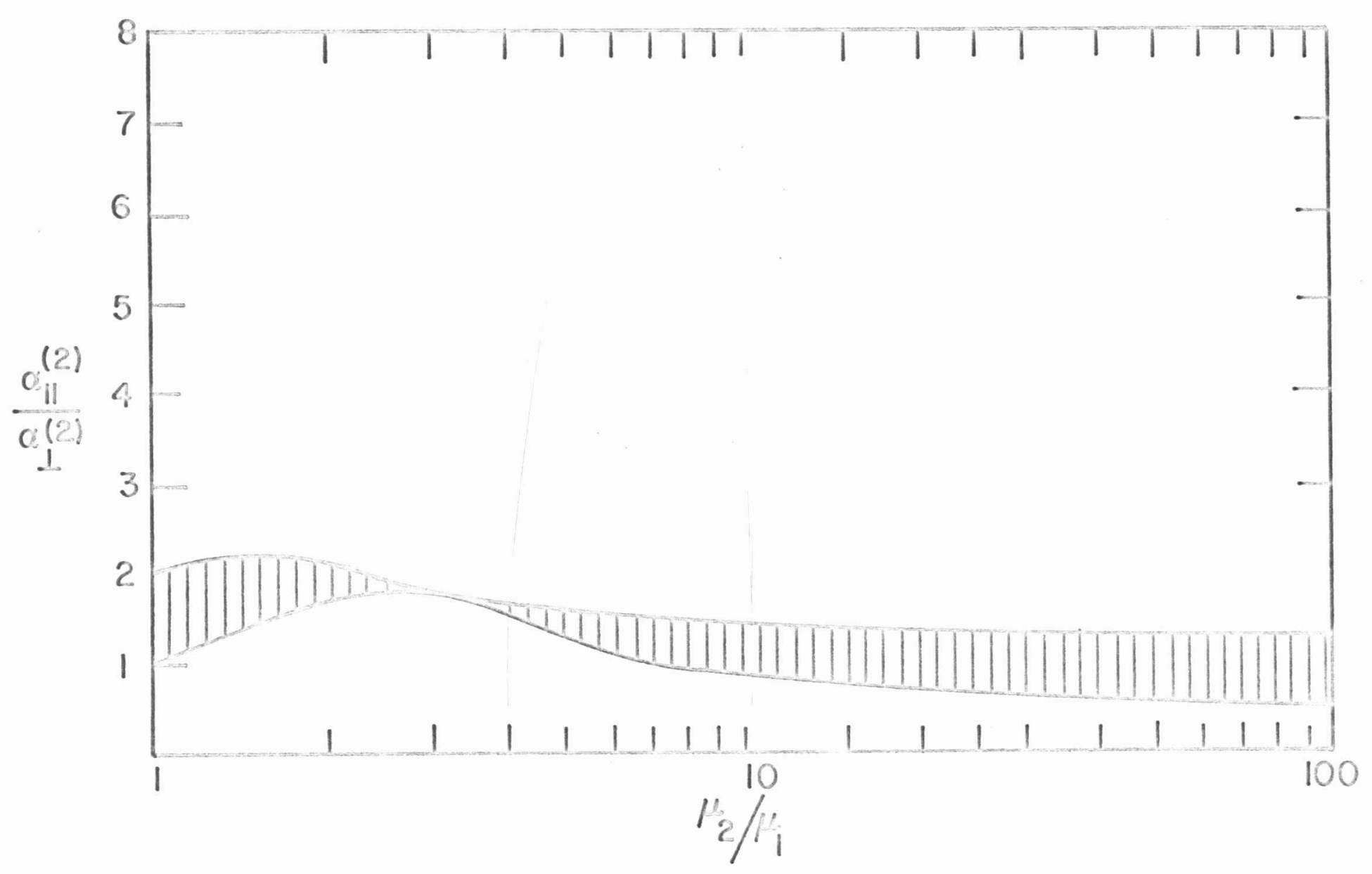

Fig.4-3. Ratio of parallel to perpenaicular polarization absorption for $\mu_{2}>\mu_{1}$ 


\section{X-point Transitions}

The preceding sections in this chapter have dealt with transitions at the $\Gamma$-point in cubic and tetrahedral crystals. If an extremum occurs at some other point where the conduction band and valence band have opposite parity, the polarization dependence will generally be different because of the non-spherical nature of the energy surfaces. In this section we will consider transitions at the $\mathrm{X}$-point in simple cubic and face-centered cubic crystals (see Fig. E-I in Appendix E.)

The group of the wave vector at $X$ is $D_{4 h}$, so that symetry dictates that the surfaces of constant energy near $\mathrm{X}$ be ellipsoids of revolution for non-degenerate bands and for bands degenerate at $X$ which remain degenerate near $\mathrm{x}$. The energy surfaces are described by

$$
\begin{aligned}
E_{n}(\vec{k}) & =E_{n}\left(\vec{k}_{0}+\vec{k}^{\prime}\right) \\
& \left.=E_{n}\left(\vec{k}_{0}\right)+\frac{\hbar^{2}}{2}\left[a\left(k_{r_{1}}\right)^{2}+a k_{r_{2}}^{\prime}\right)+b k_{r_{3}}^{\prime}\right]
\end{aligned}
$$

where $\left(r_{1}, r_{2}, r_{3}\right)$ is any permutation of $(x, y, z) ; k_{0}$ is one of the $X$-points, and

$$
\begin{aligned}
& a=\left(\frac{1}{m_{n}^{*}}\right)_{r_{1} r_{1}}=\left(\frac{1}{m_{n}^{*}}\right)_{r_{2} r_{2}} \\
& b=\left(\frac{1}{m_{n}^{*}}\right)_{r_{3} r_{3}}
\end{aligned}
$$

Fig.4-4 is a sketch of the surfaces when they are prolate spheroids, when $a>b$. The surfaces can also be oblate 


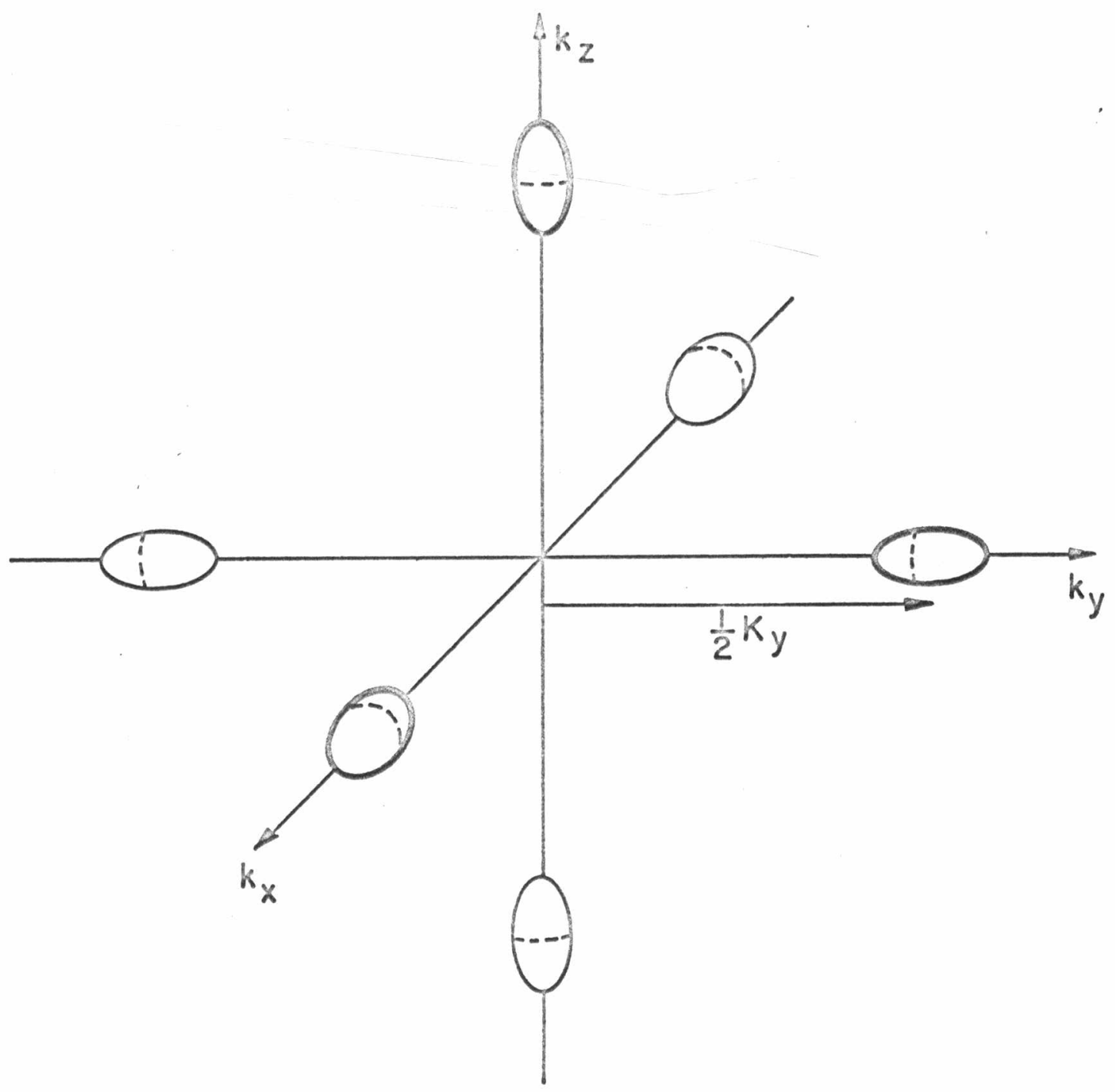

$$
\begin{array}{ll}
\text { Fig.4-4. Constant energy surfaces near } \\
\text { X-point extremum }
\end{array}
$$


spheroids, for which $a<b$. The dotted lines indicate the intersections of zone surfaces $\vec{k}=\frac{1}{2} \vec{K}_{r_{i}}$, where $\vec{K}_{r_{i}}$ is a reciprocal lattice vector. The states corresponding to the values $\vec{k}$ on the surface of each ellipsoid are equivalent to the states having value $\vec{k}-\vec{K} r_{i}$. We have therefore indicated by heavy lines the $\vec{k}$ values which we will use. The second order matrix element is

$$
\begin{aligned}
& P_{f g}^{(2)} \equiv \frac{\hbar k^{\prime}}{m} P_{c V}^{(2)}\left(k_{0}\right)= \\
& \frac{\hbar k^{\prime}}{m} \sum_{l}^{\prime}\left[\frac{\left\langle c_{i}\left(k_{0}\right) \hat{k}^{\prime} \cdot \vec{p} \mid l\left(k_{0}\right)\right\rangle\left\langle l\left(k_{0}\right)\left|\hat{\varepsilon}_{1} \cdot \vec{p}\right| c_{i}\left(k_{0}\right)\right\rangle\left\langle c_{i}\left(k_{0}\right)\left|\hat{\varepsilon}_{2} \cdot \vec{p}\right| V_{j}\left(k_{0}\right)\right\rangle}{\hbar \omega_{1}}\right. \\
& +\frac{\left\langle c_{i}\left(k_{0}\right)\left|\hat{\varepsilon}_{1} \cdot \vec{p}\right| \ell\left(k_{0}\right)\right\rangle\left\langle l\left(k_{0}\right)\left|\hat{k}^{\prime} \cdot \vec{p}\right| c_{i}\left(k_{0}\right)\right\rangle\left\langle c_{i}\left(k_{0}\right)\left|\hat{\varepsilon}_{2} \cdot \vec{p}\right| v_{j}\left(k_{0}\right)\right\rangle}{\left.\hbar \omega_{1}-E_{l}\right)} \\
& -\frac{\left\langle c_{i}\left(k_{0}\right)\left|\hat{\varepsilon}_{1} \cdot \vec{p}\right| V_{j}\left(k_{0}\right)\right\rangle\left\langle V_{j}\left(k_{0}\right)\left|\hat{k}^{\prime} \cdot \vec{p}\right| l\left(k_{0}\right)\right\rangle\left\langle l\left(k_{0}\right)\left|\hat{\varepsilon}_{2} \cdot \vec{p}\right| V_{j}\left(k_{0}\right)\right\rangle}{\hbar \omega_{2}} \\
& -\frac{\left\langle c_{i}\left(k_{0}\right)\left|\hat{\varepsilon}_{1} \cdot \vec{p}\right| V_{j}\left(k_{0}\right)\right\rangle\left\langle v_{j}\left(k_{0}\right)\left|\hat{\varepsilon}_{2} \cdot \vec{p}\right| \ell\left(k_{0}\right)\right\rangle\left\langle l\left(k_{0}\right)\left|\hat{k}^{\prime} \cdot \vec{p}\right| V_{j}\left(k_{0}\right)\right\rangle}{\left(E_{V}-E_{l}\right)} \\
& +(1 \longleftrightarrow 2)]
\end{aligned}
$$

Recalling that

$$
\begin{aligned}
\sum_{l}^{\prime} & {\left[\frac{\left\langle n\left(k_{0}\right)|\hat{k} \cdot \vec{p}| l\left(k_{0}\right)\right\rangle\left\langle l\left(k_{0}\right)|\hat{\varepsilon} \cdot \vec{p}| n\left(k_{0}\right)\right\rangle}{E_{n}-E_{l}}\right.} \\
& \left.+\frac{\left\langle n\left(k_{0}\right)|\hat{\varepsilon} \cdot \vec{p}| l\left(k_{0}\right)\right\rangle\left\langle l\left(k_{0}\right)|\hat{k} \cdot \vec{p}| n\left(k_{0}\right)\right\rangle}{E_{n}-E_{l}}\right]
\end{aligned}
$$


$=\hat{k} \cdot\left[\left(\stackrel{\frac{m^{2}}{m_{n}^{*}}}{=}-m \mathbb{1}\right] \cdot \hat{\varepsilon}\right.$

we have

$$
\begin{aligned}
& p_{C V}^{(2)}\left(k_{0}\right)=
\end{aligned}
$$

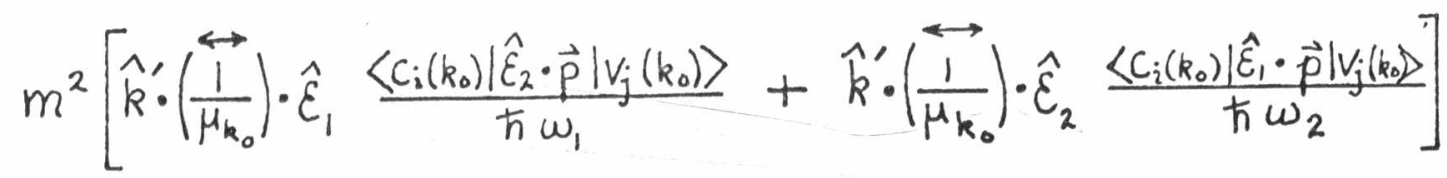

From Eq.2-7

$$
\begin{aligned}
& \alpha^{(2)}=A^{\prime} \frac{\hbar^{2}}{m^{2}} \frac{V}{(2 \pi)^{3}} \sum_{i j k_{0}} \int d^{3} k^{\prime}\left(k^{\prime}\right)^{2}\left|P_{c v}^{(2)}\left(k_{0}\right)\right|^{2} \delta\left[E_{g a p}+\frac{\hbar^{2}}{2} \vec{k}^{\prime \prime} \cdot \frac{1}{\mu_{k_{0}}} \cdot \vec{k}^{\prime}-\hbar\left(\omega_{1}+\omega_{2}\right)\right] \\
& =A^{\prime} \frac{\hbar^{2}}{m^{2}} \frac{V}{(2 \pi)^{3}} \frac{1}{2}\left(\frac{2}{\hbar^{2}}\right)^{5 / 2}\left[\hbar\left(\omega_{1}+\omega_{2}\right)-E_{g a p}\right]^{3 / 2} \sum_{i j k_{0}} \int d \Omega_{k^{\prime}} \frac{\left|P_{c v}^{(2)}\left(k_{0}\right)\right|^{2}}{\left[\hat{k}^{\prime} \cdot\left(\frac{1}{\mu_{k_{0}}}\right) \cdot \hat{k}^{\prime}\right]^{5 / 2}} \\
& (4-27)
\end{aligned}
$$

Consider the $x$-point on the $k_{z}$ axis. The matrix element $\left\langle c_{i}\left(k_{o z}\right)|\hat{\varepsilon} \cdot \vec{p}| V_{j}\left(k_{0 z}\right)\right\rangle$ is non-zero only if $x^{c} \times x^{v}$ contains $X_{2}$ or $X_{5}$. Let us suppose that $\left|V_{j}\left(k_{0 z}\right)\right\rangle=$ $\left|X_{1}\left(k_{\circ z}\right)\right\rangle$ and $\left|c_{i}\left(k_{\circ z}\right)\right\rangle=\left|X_{2}\left(k_{\circ z}\right)\right\rangle$. Then (for $\hat{\varepsilon}=$ $(1, m, n))$

$$
\begin{aligned}
\left\langle x_{2}\left(k_{0 z}\right)|\hat{\varepsilon} \cdot \vec{p}| x_{1}\left(k_{0 z}\right)\right\rangle & =n\left\langle x_{2}\left|p_{z}\right| x_{1}\right\rangle \\
& =n\langle p\rangle_{c v}
\end{aligned}
$$

If $\left|c_{i}\left(k_{o z}\right)\right\rangle=\left|x_{5 i}\left(k_{o z}\right)\right\rangle$, 
118

$$
\begin{aligned}
\left\langle x_{51}\left(k_{0 z}\right)|\hat{\varepsilon} \cdot \vec{p}| X_{1}\left(k_{0 z}\right)\right\rangle & =l\left\langle x_{51}\left|p_{x}\right| x_{1}\right\rangle \\
& =l\langle p\rangle_{c V}, \\
\left\langle x_{52}\left(k_{0 z}\right)|\hat{\varepsilon} \cdot \vec{p}| x_{1}\left(k_{0 z}\right)\right\rangle & =m\langle p\rangle_{c V} .
\end{aligned}
$$

Expanding the absolute square in Fq.4-27, the integral,

over the angles of the first term is

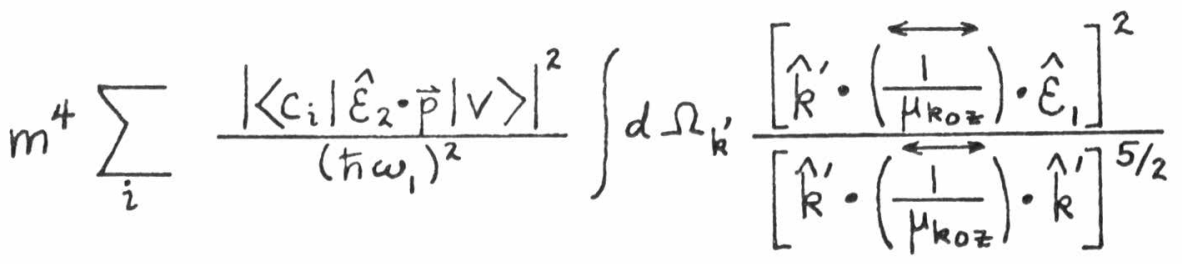

$$
\begin{aligned}
& =m^{4} \sum_{i} \frac{\left|\left\langle c_{i}\left|\hat{\varepsilon}_{z} \cdot \vec{p}\right| v\right\rangle\right|^{2}}{\left(\hbar \omega_{1}\right)^{2}} \int d \Omega_{k^{\prime}} \frac{\left(a \ell k_{x}+a m k_{y}+b n k_{z}\right)^{2}}{\left[a K_{x}^{2}+a k_{y}^{2}+b k_{z}^{2}\right]^{5 / 2}} \\
& =m^{4} \sum_{i} \frac{\left|\left\langle c_{i}\left|\hat{\varepsilon}_{2} \cdot \vec{p}\right| v\right\rangle\right|^{2}}{\left(\hbar \omega_{1}\right)^{2}} \frac{4 \pi}{3} \frac{1}{\sqrt{b}}\left[l_{1}^{2}+m_{1}^{2}+\frac{b}{a} n_{1}^{2}\right]
\end{aligned}
$$

The cross terms in the absolute square give

$$
\begin{array}{r}
\frac{m^{4}}{\left(\hbar \omega_{1}\right)\left(\hbar \omega_{2}\right)} \sum_{i}\left\{\left\langle c_{i}\left|\hat{\varepsilon}_{1} \cdot \vec{p}\right| v\right\rangle\left\langle c_{i}\left|\hat{\varepsilon}_{2} \cdot \vec{p}\right| v\right\rangle^{*}+c_{0} c \cdot\right\} \\
\quad x \int d \Omega_{k^{\prime}} \frac{\left[\hat{k}^{\prime} \cdot\left(\frac{1}{\mu_{k_{0} z}}\right) \cdot \hat{\varepsilon}_{1}\right]\left[\hat{k}^{\prime} \cdot\left(\frac{1}{\mu_{k 0 z}}\right) \cdot \hat{\varepsilon}_{2}\right]}{\left[\hat{k}^{\prime} \cdot\left(\frac{1}{\mu_{k 0 z}}\right) \cdot \hat{k}^{\prime}\right]^{5 / 2}} \\
=\frac{m^{4}}{\left(\hbar \omega_{1}\right)\left(\hbar \omega_{2}\right)} \sum_{i}\left\{\left\langle c_{i}\left|\hat{\varepsilon}_{1} \cdot \vec{p}\right| v\right\rangle\left\langle v\left|\hat{\varepsilon}_{2} \cdot \vec{p}\right| c_{i}\right\rangle+c_{0} \cdot c_{0}\right\} \\
\quad \times \frac{4 \pi}{3} \frac{1}{\sqrt{b}}\left[l_{1} l_{2}+m_{1} m_{2}+\frac{b}{a} n_{1} n_{2}\right]
\end{array}
$$


Similar expressions result for the two other $\mathrm{X}$-points, with $I$ and $n$ permuted and $m$ and $n$ permuted. Using these expressions and Eq.4-28 and Eq.4-29 we obtain $\mathrm{x}_{1}^{ \pm} \longleftrightarrow \mathrm{x}_{2}^{\mp}$

$$
\begin{array}{r}
\alpha^{(2)=} B^{\prime} \frac{1}{\sqrt{6}}\left\{\left(\hat{\varepsilon}_{1} \cdot \hat{\varepsilon}_{2}\right)^{2}\left[\frac{1}{\hbar \omega_{1}}+\frac{1}{\hbar \omega_{2}}\right]^{2}+\left(\hat{\varepsilon}_{1} \times \varepsilon_{2}\right)^{2}\left[\left(\frac{1}{\hbar \omega_{1}}\right)^{2}+\left(\frac{1}{\hbar \omega_{2}}\right)^{2}\right]\right. \\
\left.b+\left[l_{1}^{2} l_{2}^{2}+m_{1}^{2} m_{2}^{2}+n_{1}^{2} n_{2}^{2}\right]\left[\frac{b}{a}-1\right]\left[\frac{1}{\hbar \omega_{1}}+\frac{1}{\hbar \omega_{2}}\right]^{2}\right\}
\end{array}
$$

$\mathrm{x}_{1}^{ \pm} \longleftrightarrow \mathrm{x}_{5}^{\mp}$

$\alpha^{(2)}=B^{\prime} \frac{1}{\sqrt{b}}\left\{\left(\hat{\varepsilon}_{1} \cdot \hat{\varepsilon}_{2}\right)^{2}\left[\frac{b}{a}+1\right]\left[\frac{1}{\hbar \omega_{1}}+\frac{1}{\hbar \omega_{2}}\right]^{2}\right.$

$$
\begin{array}{ll}
b & +\left(\hat{\varepsilon}_{1} \times \hat{\varepsilon}_{2}\right)^{2}\left[\frac{b}{a}+1\right]\left[\left(\frac{1}{\hbar \omega_{1}}\right)^{2}+\left(\frac{1}{\hbar \omega_{2}}\right)^{2}\right] \\
- & \left.\left[l_{1}^{2} l_{2}^{2}+m_{1}^{2} m_{2}^{2}+n_{1}^{2} n_{2}^{2}\right]\left[\frac{b}{a}-1\right]\left[\frac{1}{\hbar \omega_{1}}+\frac{1}{\hbar \omega_{2}}\right]^{2}\right\}
\end{array}
$$

where $B^{\prime}$ is defined in Eq.4-16.

When a forbidden transition occurs at the $\mathrm{X}$-point the absorption coefficient is seen to have two important features. It depends on the symmetries of the valence and conduction bands, and it depends on the orientation of the polarization vectors with respect to the crystal axes. In the limit $b=a, i . e .$, spherical energy surfaces, the absorption coefficient reduces to the form given in Eq.4-15 for $\Gamma$-point transitions. 


\section{Chapter V}

\section{Application to Experiment}

The optical spectra of solids generally show complicated structures over wide ranges of energy, and the interpretation of the various peaks and steps in an absorption spectrum aids in understanding the electronic structure of a material. Studies of the polarization dependence of two-photon absorption are aimed at examining the symmetry properties of the electronic states responsible for the absorption. This chapter contains a brief review of some two-photon absorption experiments in which polarization studies have been made and includes an examination of the experimental data in terms of the theory discussed in Chapters III and IV. The first two materials (CuCl and ZnS) crystallize in structures without inversion symmetry, and so one expects to discuss the polarization dependences in terms of allowed transitions. The last four (RbI, CsI, $K I$, and $T I C I)$ have inversion invariant structures in which the valence band and conduction band states at the band minima are of opposite parity, indicating forbidden transitions near the absorption edge. 
A. $\quad \underline{\mathrm{CuCl}}$

Two-photon measurements on $\mathrm{CuCl}$ were made by Fröhlich, Staginnus and Schönherr, 13 who for the first time reported sharp exciton lines in a two-photon spectrum. CuCl has $\mathrm{T}_{\mathbf{d}}$ point group symmetry (which does not contain the inversion) and therefore there are no parity-forbidden transitions. The band gap, according to the energy band calculations of Song ${ }^{58}$ (see Fig.5-1), is $3.43 \mathrm{eV}$, neglecting spin-orbit coupling, and occurs at the $\Gamma$-point. Spin-orbit coupling splits the valence band at $\Gamma$ by $0.072 \mathrm{ev.58}$ The twophoton spectrum of Fröhlich, Staginnus and Schönherr is shown in Fig.5-2. Cardona 59 observed relatively weak onephoton absorption in the region above the exciton lines, in contrast to the strong two-photon absorption in this region. The polarization dependence of the two-photon absorption was studied by Fröhlich et al. at the energy of the second exciton $(3.29 \mathrm{eV})$ and in the continuum region $(3.5 \mathrm{eV})$. Their results for the exciton line are shown in Fig.5-3; they did not report their results for $3.5 \mathrm{eV}$, but stated that they were different. In Fig.5-3 $\theta$ is the angle in a (100) plane between the probe polarization and a [100] axis. Parts (a) and (b) give the absorption coefficient as a function of $\theta$ with the laser polarized at $0^{\circ}$ and $135^{\circ}$, respectively. The data points in (a) are seen to be described by $\sin ^{2} \theta$, and in (b) by $\frac{1}{2}(1-\sin \theta)$. The only angular function which has this behavior is $T_{2}\left(\hat{\varepsilon}_{1}, \hat{\varepsilon}_{2}\right)$, 
which is the angular dependence expected for a $T_{2}$ valence band and $A$, conduction band. Actually the valence band is spin-orbit split into a $\Gamma^{7}$ band and a $\Gamma^{8}$ band, indicating an angular dependence $\mathrm{E}\left(\hat{\varepsilon}_{1}, \hat{\varepsilon}_{2}\right)+\mathrm{cT}\left(\hat{\varepsilon}_{1}, \hat{\varepsilon}_{2}\right)+$ $\mathrm{c}^{\prime} \mathrm{T}_{2}\left(\hat{\varepsilon}_{1}, \hat{\varepsilon}_{2}\right)$ for the $s$ exciton derived from $\Gamma^{8}$ and $\Gamma^{6}\left(\Gamma^{\prime}\right)$ states (see Table 3-I). However, the spin-orbit coupling is small, so that the $\mathrm{T}_{2}$ contribution is expected to dominate.

In the one-photon measurements of Cardona ${ }^{59}$, the region above $3.35 \mathrm{eV}$ is smooth and relatively flat, indicating small absorption at the $\mathrm{X}$-point. Song ${ }^{58}$ has calculated the electric dipole moments for transitions $\mathrm{T}_{2} \rightarrow \mathrm{A}_{1}(3.43 \mathrm{eV})$ and $\mathrm{X}_{1}, \mathrm{X}_{5} \rightarrow \mathrm{X}_{3}{ }^{60}(3.77 \mathrm{eV}$ and $3.84 \mathrm{eV})$. He finds

$$
\frac{\left|\langle\vec{r}\rangle_{r}\right|^{2}}{\left|\langle\vec{r}\rangle_{x}\right|^{2}} \approx 30
$$

which he suggests to be the reason for the lack of structure in the continuum of Cardona's data. For twophoton absorption one needs the matrix elements

$$
\sum_{l} \frac{\left\langle A_{1}|\vec{p}| \ell\right\rangle\left\langle\ell|\vec{p}| T_{2}\right\rangle}{E_{l}-E_{T_{2}}-\hbar \omega} \text { and } \sum_{l} \frac{\left\langle x_{1}|\vec{p}| \ell\right\rangle\left\langle\ell|\vec{p}| X_{3}\right\rangle}{E_{l}-E_{x_{3}}-\hbar \omega} \text {. }
$$

The nearest intermediate states at $\vec{k}=0$ which connect $T_{2}$ and $A_{1}$ are the lower valence band states of symmetry $T_{2}$ and the upper conduction band states of symmetry $\mathrm{T}_{2} \cdot$ On the other hand, the state at $X_{5}$ immediately below $X_{1}$ 
connects $X_{1}$ and $X_{3}$. Thus one might find that $X$-point transitions give a larger contribution to the absorption in the two-photon spectrum than in the one-photon spectrum. If the strong absorption observed by Fröhlich, Staginnus and Schönherr above $3.35 \mathrm{eV}$ is due in part to $\mathrm{X}$-point excitons and continuum transitions, one would expect a polarization dependence different than that observed for the exciton at $3.29 \mathrm{eV}$. However, the absence of nearby connecting intermediate states at $\Gamma$ indicates that "forbidden" transitions (i. e. taking place away from the $\Gamma$-point, in the band-to-band picture) might give a larger contribution to the absorption than "allowed" transitions. Mahan 20 has previously suggested (in the more correct exciton picture) that p-state absorption would be stronger than s-state absorption in the continuum. If this forbidden absorption is larger than the $\mathrm{X}$-point allowed absorption, the polarization dependence would be given by Eq.4-8 or Eq.4-15, again quite different from that of the $3.29 \mathrm{eV}$ exciton. 


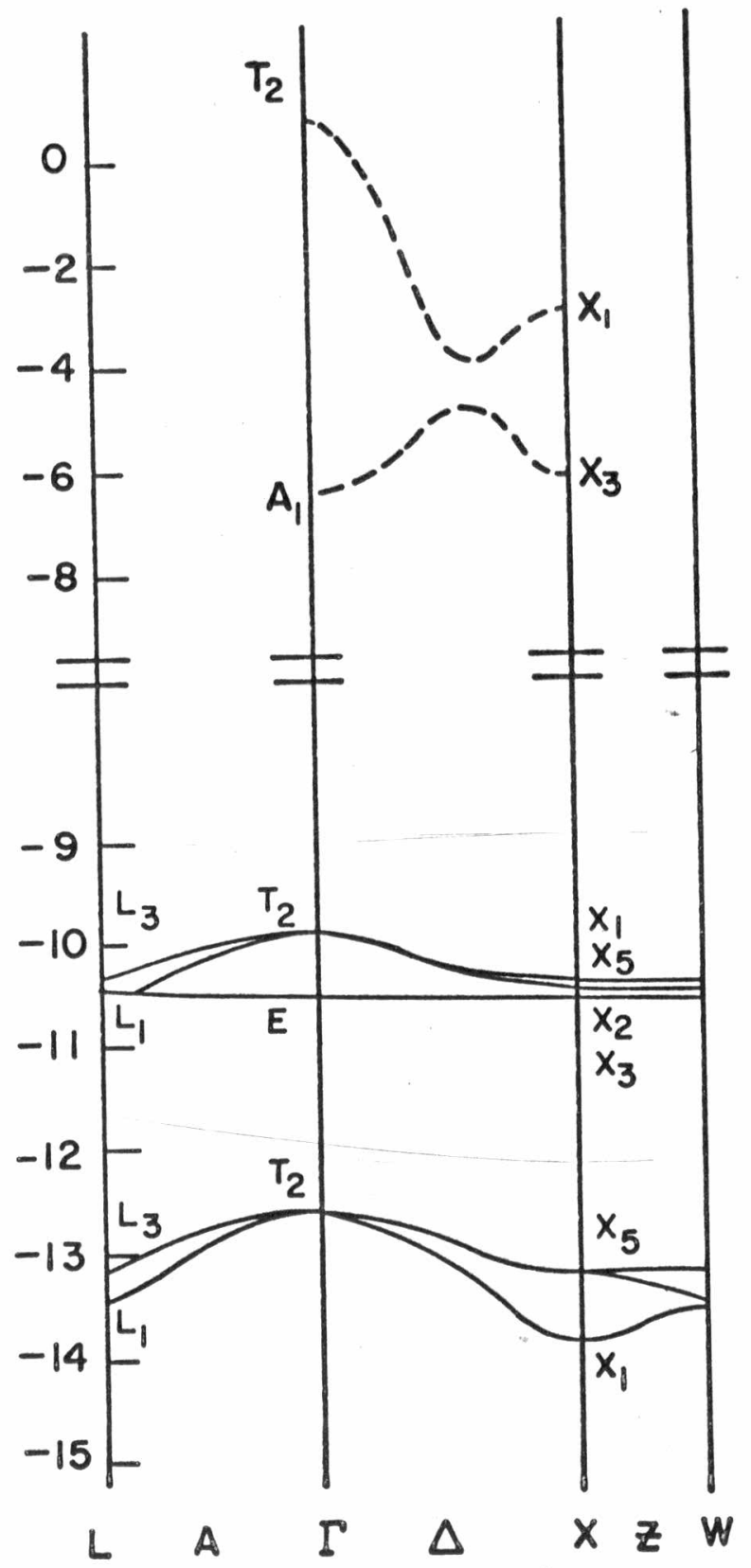

Fig.5-1. Band structure of CuCl (after Song, Ref. 58) 


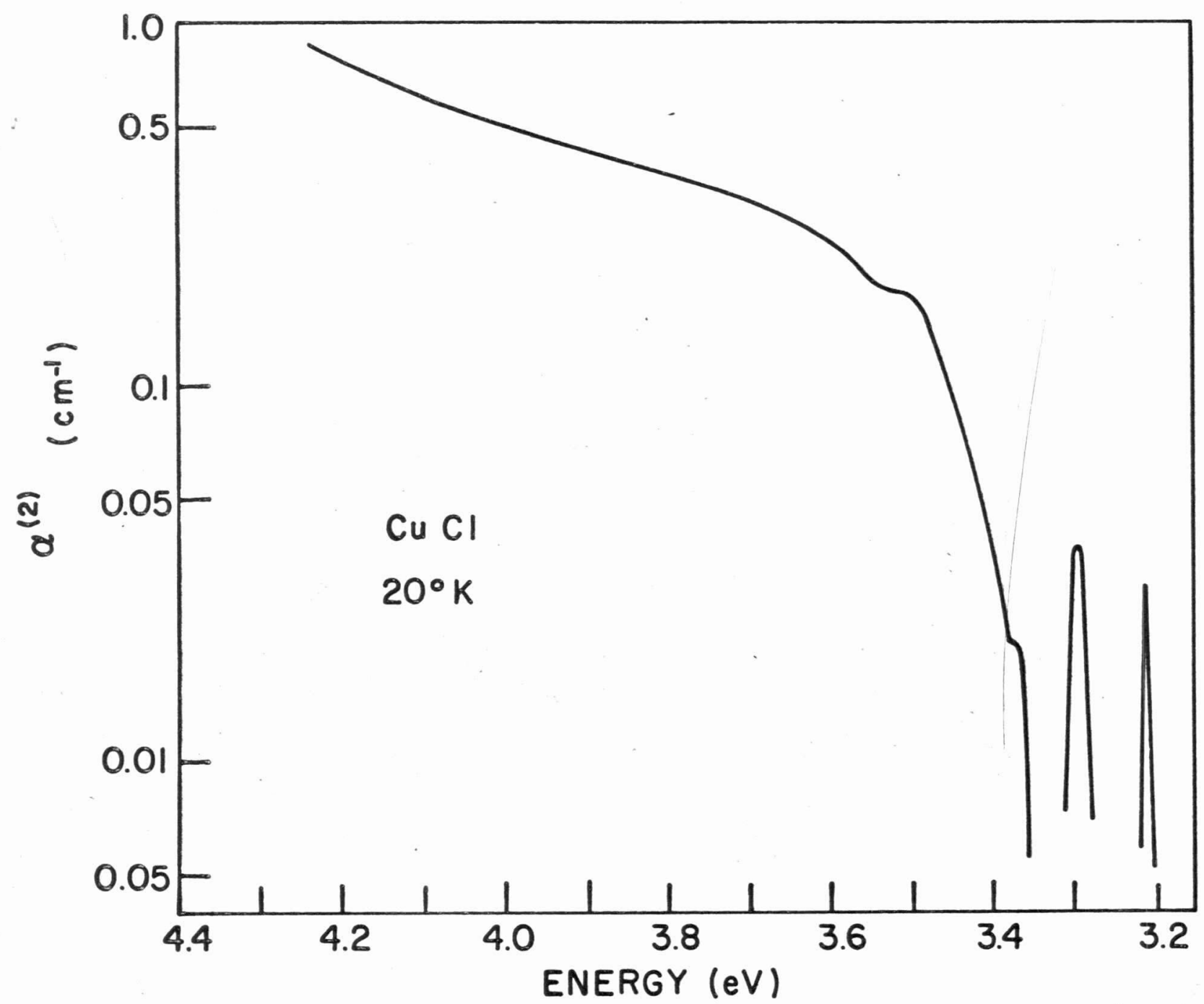

Fig.5-2. Two-photon absorption spectrum of CuCl (after Frohlich, Staginnus and Schonherr, Ref. 13) 


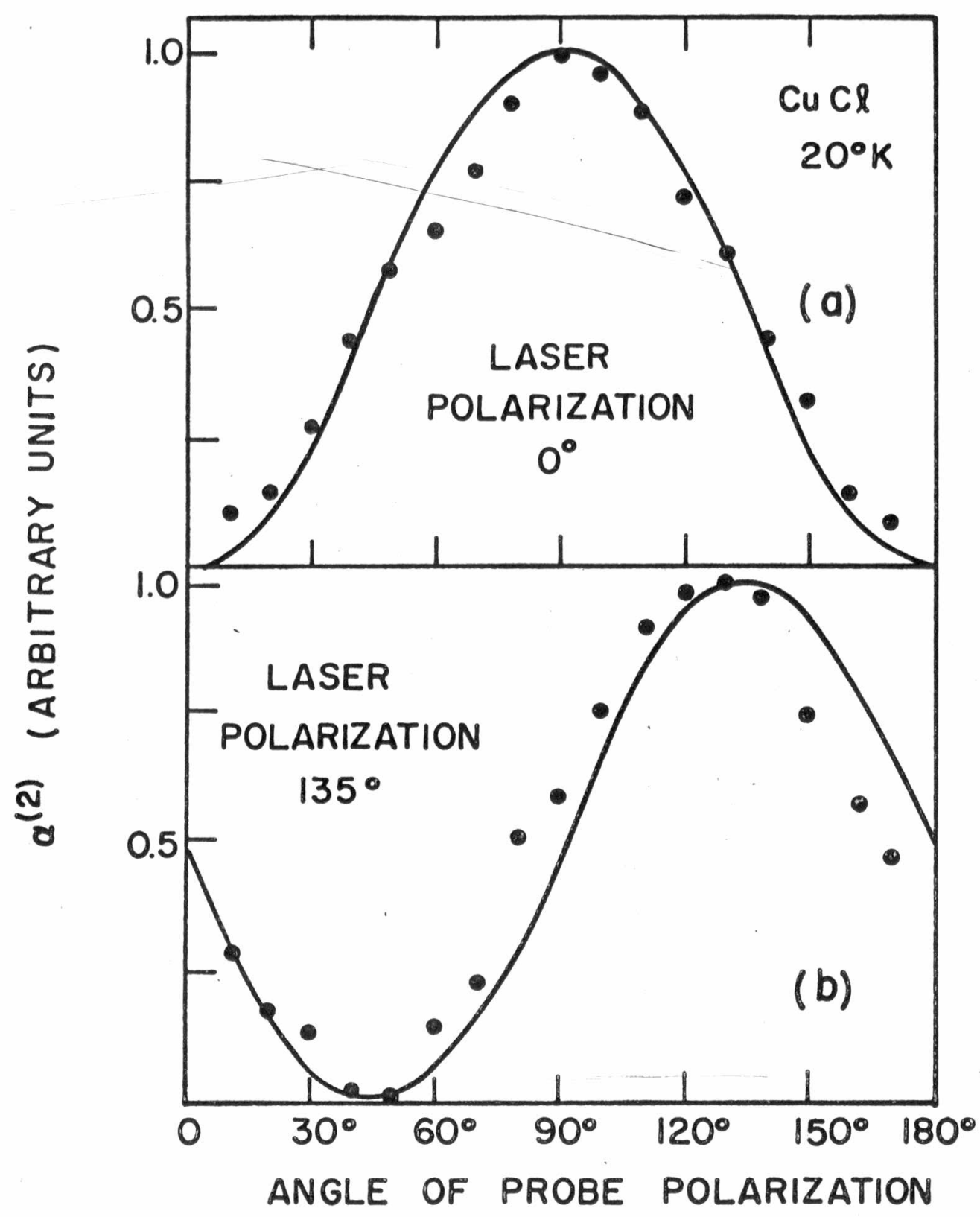

Fig.5-3. Polarization dependence of two-photon absorption in CuCl (after Frohlich, Staginnus and Schonherr, Ref. 13) 


\section{B. $\mathrm{ZnS}$}

ZnS crystallizes in the zinc blende $\left(T_{d}\right)$ and wurtzite $\left(C_{6 V}\right)$ structures, of which the wurtzite form has been the subject of two-photon absorption measurements. 61,17

Energy bands for wurtzite ZnS have been calculated by Bergstresser and Cohen 62 and are shown in part in Fig.5-4. These energy bands were calculated ignoring spin-orbit interactions. With the inclusion of spin the lowest conduction band at $\Gamma$ is $\Gamma^{7}$, while the uppor valence bands are $\Gamma^{9}, \Gamma^{7}$, and $\Gamma^{7}$, in order of decreasing energy, resulting primarily from combined crystal field and spin-orbit splitting of the $3 p$ states of the sulfur ions. In Fig.5-4 the energy separation between valence bands $\Gamma^{1}$ and $\Gamma^{6}$ ( $E_{1}$ in the notation of Table 2-I) is $0.05 \mathrm{eV}$, while the spin-orbit splitting of these states in the absence of the crystal field interaction is $0.09 \mathrm{eV} .62,63$ The relative magnitudes of these energies indicate that the spin-orbit coupling can not be ignored, as it could be for CuCl, in favor of crystal field splitting alone. This conclusion was drawn by Thomas and Hopfield 64 regarding the valence bands in CdS, which has a band structure closely resembling that of ZnS. 62 This is important in discussing selection rules for optical absorption.

The two-photon spectrum of wurtzite $\mathrm{ZnS}$ has been investigated by Park and $W^{6} f^{61}$ and by Reilly. 17 Park and Waff measured the absorption coefficient up to about 
$4 \mathrm{eV}$ at liquid helium temperature, but they reported no measurements of polarization dependence. Reilly measured the absorption coefficient from $4 \mathrm{eV}$ to $4.4 \mathrm{eV}$, and the polarization dependence, given in Fig.5-5, at (a) $4.05 \mathrm{eV}$ and (b) $4.15 \mathrm{eV}$. From Table $3-1$ we find that the $\Gamma^{9} \rightarrow \Gamma^{7}$ transition is a constant with respect to $\theta$, the laser. polarization, in both case A (probe polarized in $x y$ plane) and case $B$ (probe polarized along the $z$ axis). The $\Gamma^{7} \rightarrow \Gamma^{7}$ transitions, however, behave in $\operatorname{case} A$ as $c_{1} \sin ^{2} \theta+c_{1} \cos \theta$ where $c_{1}$ and $c_{2}$ are positive constants, and in case $B$ as a constant. The transitions at both $4.05 \mathrm{eV}$ and $4.15 \mathrm{eV}$ include $\Gamma^{9} \rightarrow \Gamma^{7}$ and $\Gamma^{7} \rightarrow \Gamma^{7}$ transitions. Since the $\Gamma^{9}$ valence band is the highest, the $\Gamma^{7} \rightarrow \Gamma^{7}$ transitions would be expected to give a larger contribution to the absorption at $4.15 \mathrm{eV}$ than at $4.05 \mathrm{eV}$. This is confirmed in the more pronounced sinusoidal behavior at $4.15 \mathrm{eV}$ in the A case. The possibility that forbidden transitions (wave vector dependent) are responsible for the two-photon absorption can be disregarded, in spite of the fact that the polarization dependence would be qualitatively the same, because forbidden transitions predict a slightly lower ratio of maximum to minimum at the higher energy, in contrast to the increase observed in this ratio. 


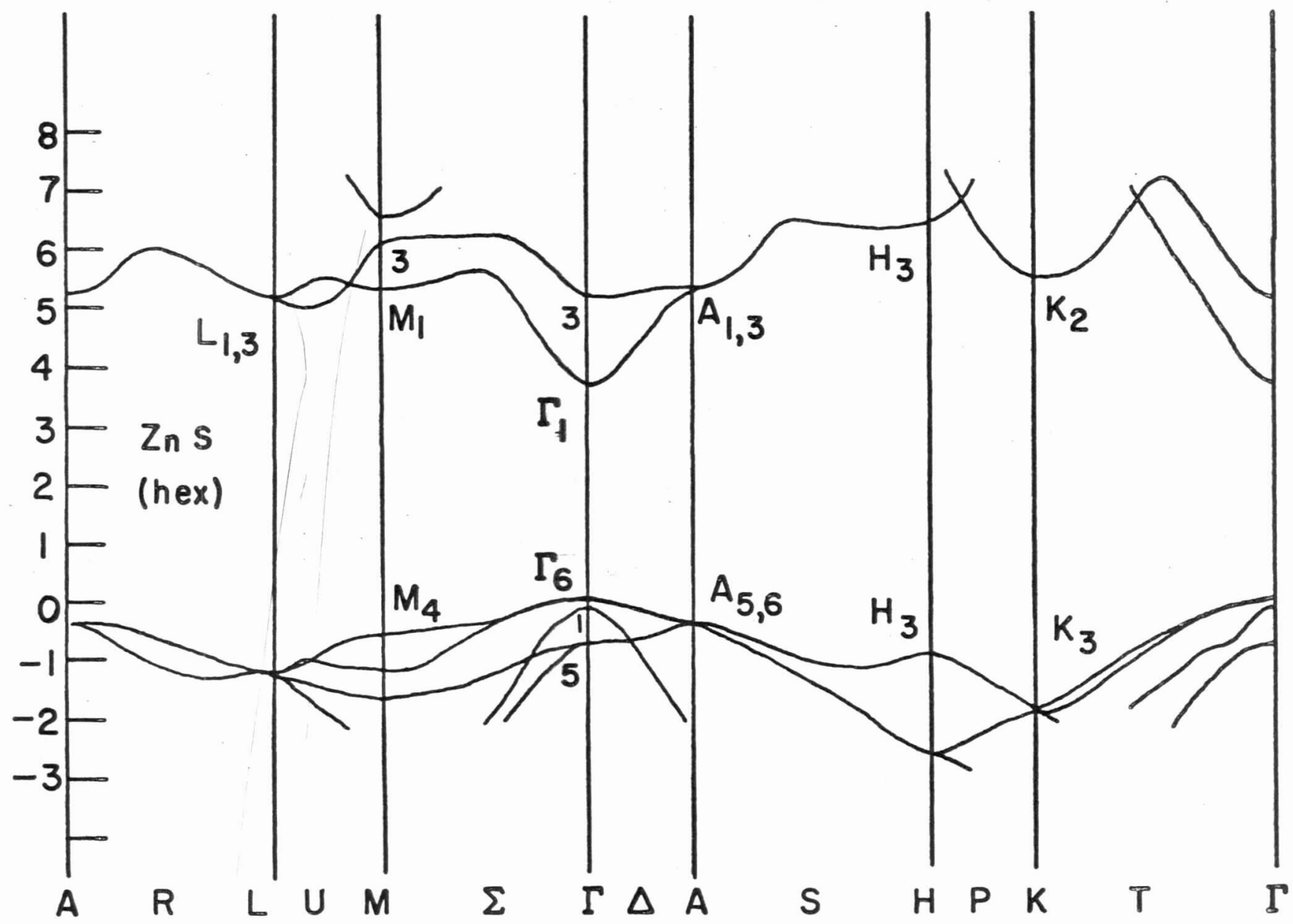
Fig.5-4. Band structure of ZnS (after Bergstresser
and Cohen, Ref. 62) 

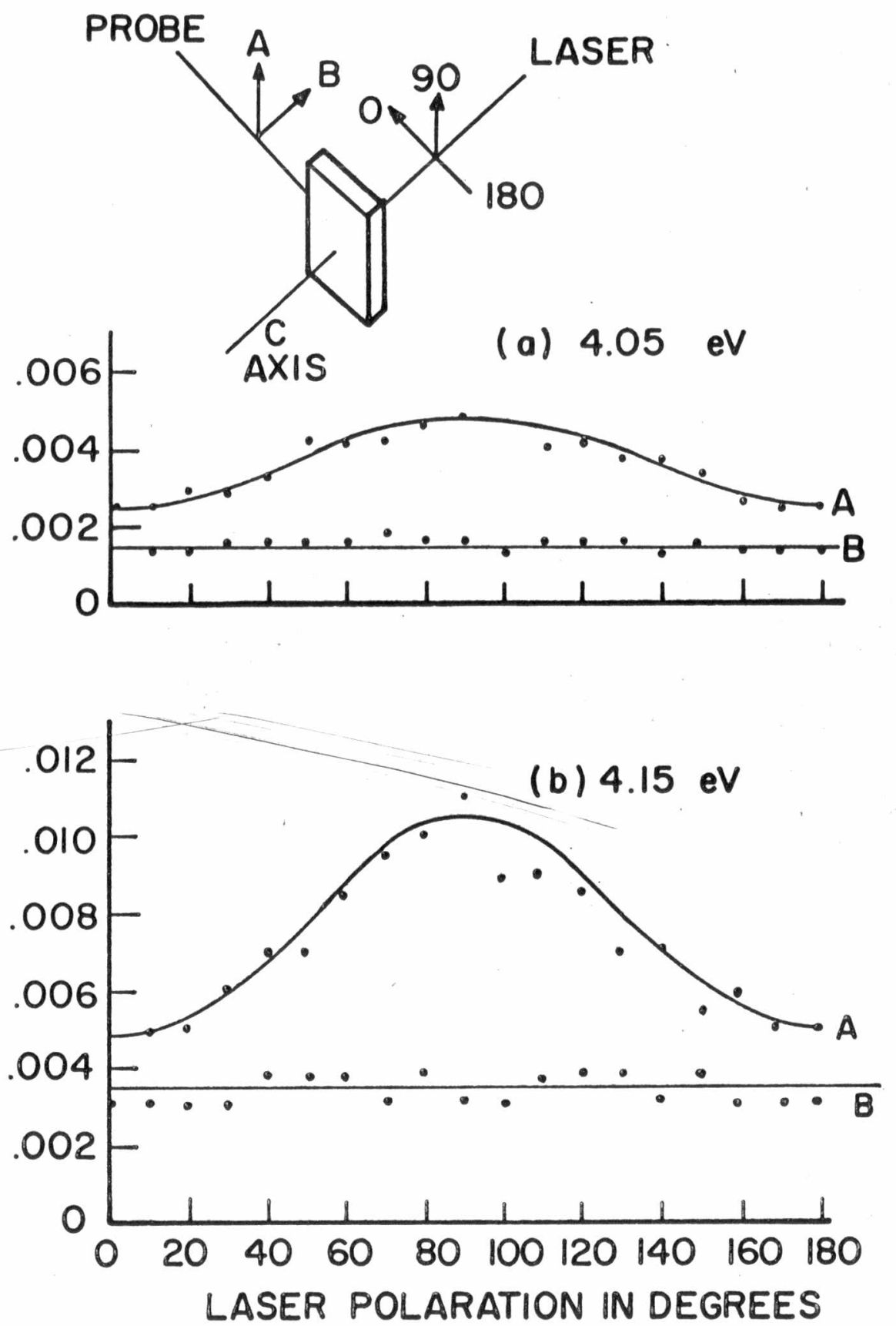

Fig.5-5. Polarization Dependence of two-photon absorption in $\mathrm{ZnS}$ at a) $4.05 \mathrm{eV}$ b) $4.15 \mathrm{eV}$ (after Reilly, Ref. 17) 
C. $\underline{R b I}$

Fröhlich and Staginnus ${ }^{12}$ measured the two-photon absorption spectrum of RbI and several alkali bromides, which have point group $O_{h}$. Since $O_{h}$ contains the inversion, the energy bands have well-defined parity at some points in the Brillouin zone. Generally in alkali halides the uppermost valence bands are derived chiefly from the p-states of the halide ion, becoming $\Gamma_{-}^{8}$ and $\Gamma_{-}^{6}$ at $\vec{k}=0$. The lowest conduction band is composed mostly of alkali s-states, which become $\Gamma_{+}^{6}$ at $\vec{k}=0.65$ since the minimum vertical energy gap is usually assumed to be at the center of the zone, the two-photon absorption near the edge can be discussed in terms of the forbidden transitions of Chapter IV, assuming, of course, that the two band model is valid and that the highest valence band $\left(\Gamma_{-}^{8}\right)$ has the well-behaved properties required.

The two-photon spectrum of Fröhlich and Staginnus for $\mathrm{RbI}$ is given in Fig.5-6 with the one-photon absorption measurements of Teegarden and Baldini. 66 Also in Fig.5-6 is the polarization dependence of the two-photon absorption for RbI at $6.8 \mathrm{eV}$. Frölich and Staginnus used a ruby laser $(1.78 \mathrm{eV})$ for one of their sources, and a conventional flash lamp for the other $(6.8 \mathrm{eV}-1.78 \mathrm{eV}=5.02 \mathrm{eV})$. The polarization dependence of Fig.5-6 is seen to have the predicted behavior; that is, $\left(\hat{\varepsilon}_{1} \cdot \hat{\varepsilon}_{2}\right)^{2}$ and a smaller amount of $\left(\hat{\varepsilon}_{1} \times \hat{\varepsilon}_{2}\right)^{2}$, with the ratio of minimum to maximum 
between $\frac{1}{2}$ and 1 . More precisely this ratio is estimated from the data to be about 0.625 . Mahan ${ }^{19}$ has calculated the theoretical value from Eq.4-9 to be

$$
\frac{\left|J_{1}\right|^{2}+\left|J_{2}\right|^{2}}{\left|J_{1}+J_{2}\right|^{2}}=0.69
$$

In the simple band model using the above energies of the photons we obtain from Eq.4-17

$$
\frac{\left(\frac{1}{\hbar \omega_{1}}\right)^{2}+\left(\frac{1}{\hbar \omega_{2}}\right)^{2}}{\left(\frac{1}{\hbar \omega_{1}}+\frac{1}{\hbar \omega_{2}}\right)^{2}}=0.613
$$

As we have said in Chapter IV, the closer agreement of the simple band model could be due to errors in the values of the exciton binding energy and the band gap and a possible error due to the sum over intermediate Wannier s-states in the evaluation of $J$, or in this single instance to accident. 

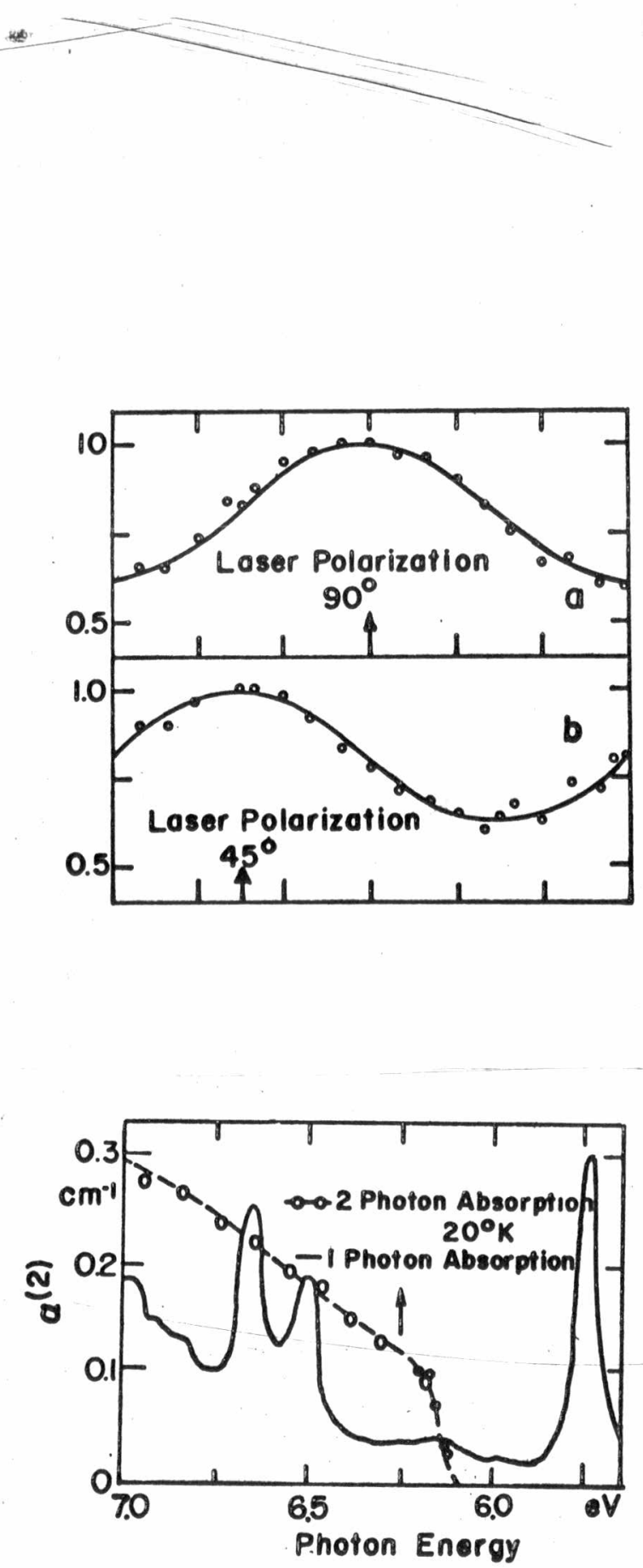

Fig.5-6. Polarization dependence of two-photon absorption and two-photon spectrum of RbI (after Frohlich and Staginnus; Ref. 12). One-photon spectrum is from Teegarden and Baldini, Rer. 66. 


\section{D. $\mathrm{CsI}$}

CsI has the simple cubic $\mathrm{CsCl}$ structure and therefore has point group symmetry $O_{h}$. Fröhlich and staginnus ${ }^{15}$ measured the two-photon absorption spectrum of CsI shown in the lower diagram of Fig.5-7, superimposed on the onephoton spectrum measured by Teegarden and Baldini. 66 The upper diagram shows the ratio of $\alpha_{\|}^{(\lambda)}$ to $\alpha_{\perp}^{(2)}$ measured by Fröhlich and Staginnus. Note that this ratio is larger than two over most of the spectrum. This disagreement with the theoretical ratio of $\operatorname{Mahan}^{20}$, as pointed out by Fröhlich and Staginnus, and of the simpler band model is probably due to the proximity of the $\Gamma_{f}^{8}\left(E_{g}\right)$ and $\Gamma_{f}^{6}\left(A_{i g}\right)$ conduction bands (see the CsI band structure, calculated by onodera, 67 in Fig.5-8). As we said in Chapter IV, a second conduction band would have to be kept in the intermediate states, and the fact that one is of $\mathrm{Eg}$ symmetry invalidates the assumption of a spherical valence band.

The two two-photon peaks at $6.25 \mathrm{eV}$ and $6.42 \mathrm{eV}$ superimposed on the band absorption have been assigned by Fröhlich and Staginnus to s excitons associated with $X_{7}^{-}$and $X_{7}^{-}\left(X_{3}\right)$, since the only states of the same parity in the correct energy range occur at $\mathrm{X}$. 


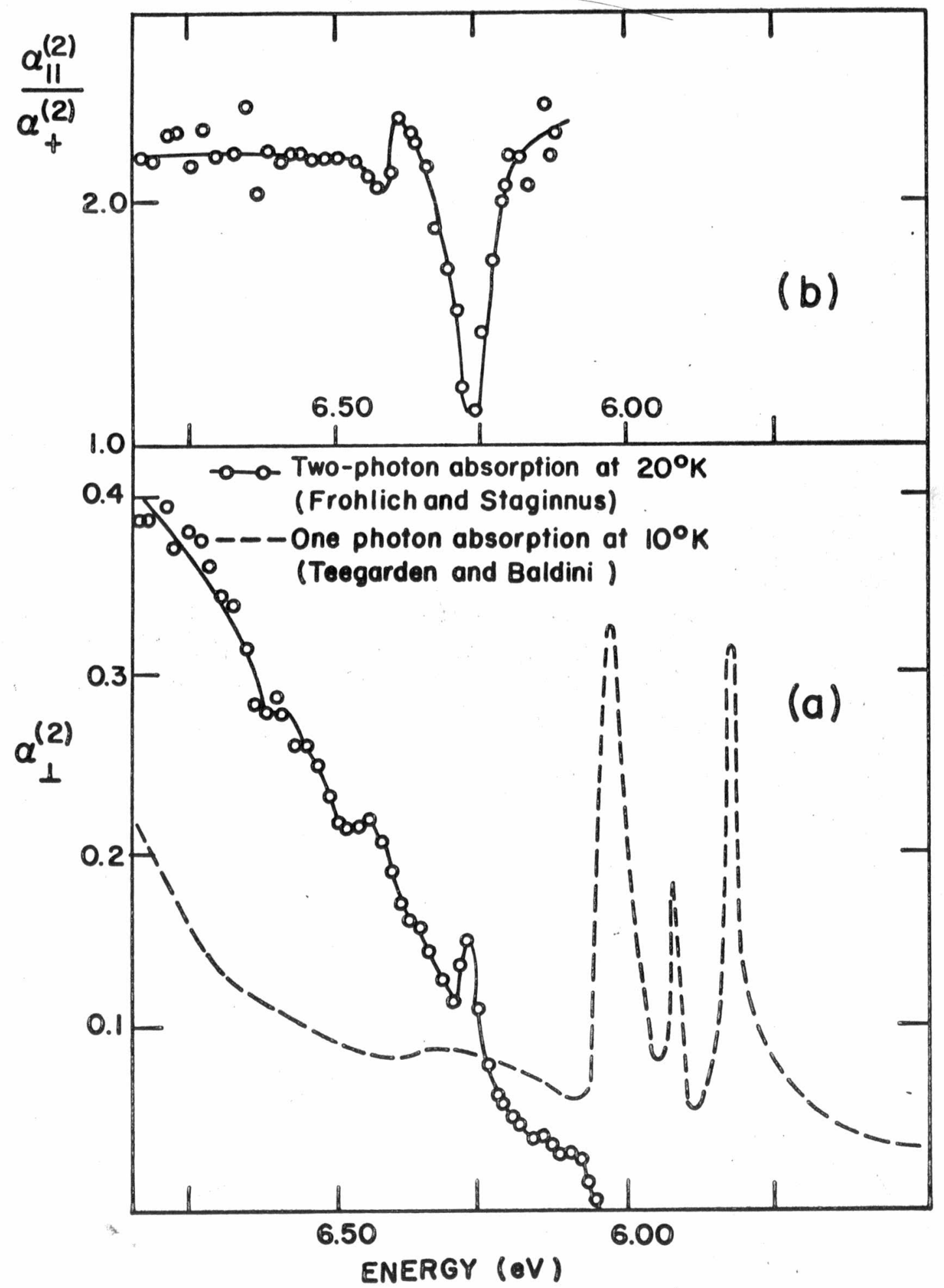

Fig.5-7. One- and two-photon absorption in CsI 


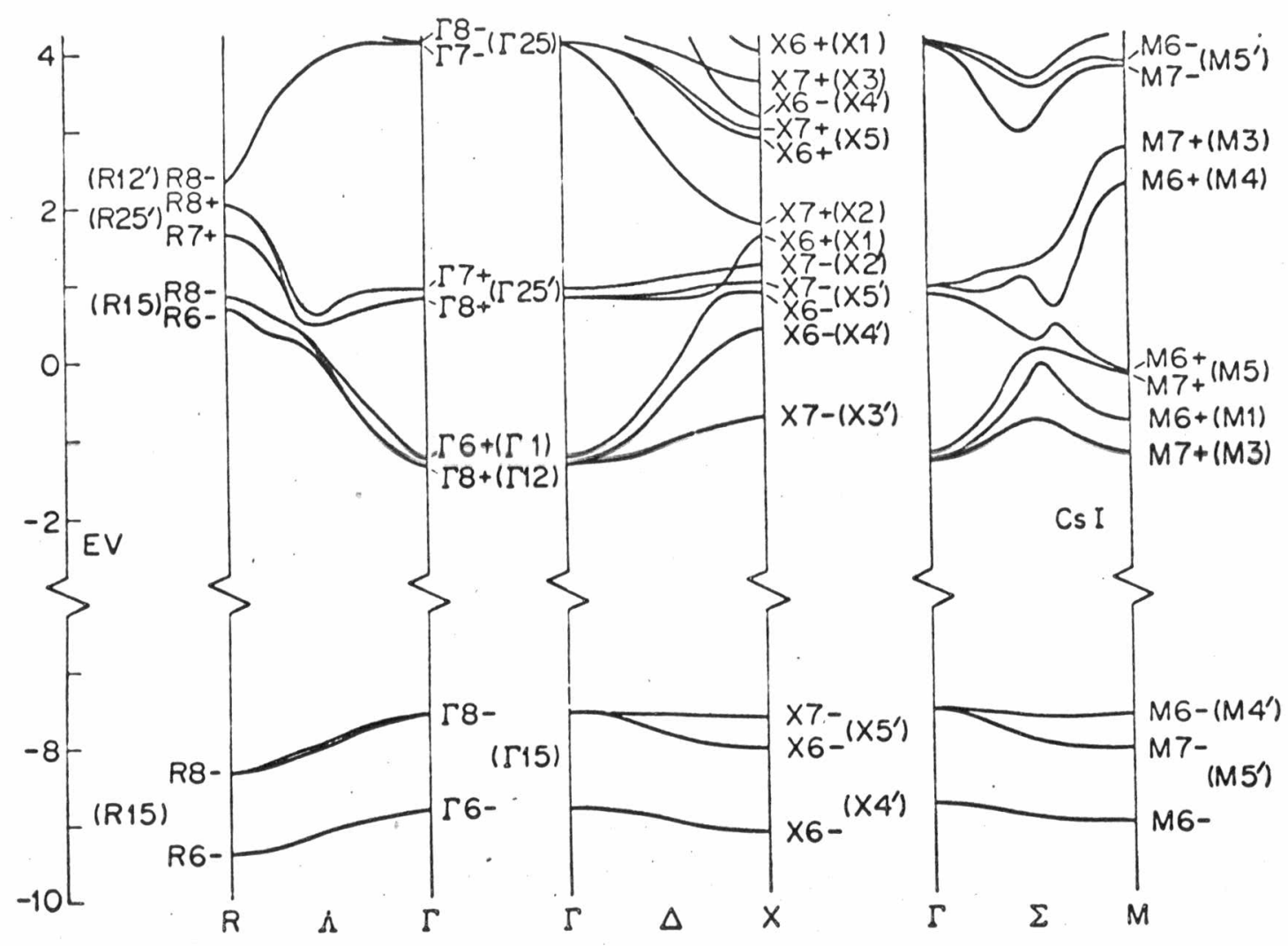

Fig.5-8. Band structure of CsI (after Onodera, Ref. 67) 
E. $\underline{K I}$

KI is face centered cubic (point group $O_{h}$ ). Energy bands have been calculated by Onodera, Okazaki and Inui ${ }^{5 l}$ and are shown in Fig.5-9. The first two-photon spectroscopic experiments on KI were performed by Hopfield, Worlock and Park ${ }^{68}$ and Hopfield and Worlock, 4 who measured the two-photon absorption up to about $6.4 \mathrm{eV}$. Park and Stafford ${ }^{16}$ have extended the measurements to $7.5 \mathrm{eV}$. Their data is shown in Fig.5-10 along with the one-photon measurements of Teegarden and Baldini. 66 Park and Stafford report two peaks in the spectrum, one at $7.21 \mathrm{eV}$, the other at $7.375 \mathrm{eV}$. Their attention is focused on the lower energy peak (the second peak will wait until a later date due to difficulties in measurement at $7.375 \mathrm{eV}$ ). Park and Stafford believe that the absorption peak at 7.21 results from the same states which produce the one-photon peak at $7.24 \mathrm{eV}$, due to their similarity in shapes and their proximity in energy. Under the assumption that the two peaks $(7.21 \mathrm{eV}$ and $7.24 \mathrm{eV})$ have the same origin, they argue that the absorption can not be due to an $s$ exciton associated with $\Gamma_{-}^{b}$ and $\Gamma_{+}^{b}$ bands, as suggested by Onodera et al. ${ }^{51}$ and Teegarden et al., ${ }^{66}$ since parity would not allow two-photon absorption to this state. On the same basis they discard the possibility of transitions at $X$. $\sum, K, S$ and $\Lambda$ lines are disregarded because of unsuitable energy differences and densities of states (see the 
energy band calculations of onodera et al. in Fig.5-9), and hence they conclude that the transitions producing the peak at $7.21 \mathrm{eV}$ occur on $\Delta$. In order to obtain more information about the absorption Park and Stafford measured the polarization dependence at $7.1 \mathrm{eV}$, which is reproduced in the upper diagram of Fig.5-11. The absorption appears to have a $\cos ^{2} \alpha$ dependence, where $\alpha$ is the angle between laser and probe polarization vectors. Since all the angular functions of $o_{h}$ except $A_{1} g\left(\hat{\varepsilon}_{1}, \hat{\varepsilon}_{2}\right)$ have dips when $\hat{\varepsilon}_{1} \cdot \hat{\varepsilon}_{2}=1$, they conclude that $\Delta_{6} \rightarrow \Delta_{6}$ is the correct choice, this being the only $\Delta$ transition which contains an $A_{1 g}$ dependence. Park and Stafford seem to be in error here; $\Delta_{7} \rightarrow \Delta_{7}$ also contains an $A_{1 g}\left(\hat{\varepsilon}_{1}, \hat{\varepsilon}_{2}\right)$ function, as can be seen in Table E-I and Table 3-I for $C_{4 V}$.

There appears, however, to be another possibility. Suppose the transition takes place between the ellipsoidal surfaces about $\mathrm{X}_{7}^{-}$and $\mathrm{x}_{7}^{+}$. In Chapter IV we calculated the angular dependence functions for $x_{1}^{ \pm} \rightarrow x_{2}^{\mp}$ and $\mathrm{x}_{1}^{ \pm} \rightarrow \mathrm{x}_{5}^{\mp}$ transitions. The transition $\mathrm{x}_{7}^{-} \rightarrow \mathrm{x}_{7}^{+}$would have as an angular dependence a linear combination of those functions, since $X_{7}^{-} \times X_{7}^{+}$contains $X_{2}$ and $X_{5}$ (see, for example, Koster et al. ${ }^{42}$ ). In Fig.5-9 it can be seen that the effective mass of $\mathrm{x}_{7}^{+}$is larger along $\Delta$ than along $\Sigma$, so that in Eq.4-30 and Eq.4-31,a $>$ b. The precise values of $a$ and $b$ are not known, but for $a=2 b$ we have plotted the angular functions for $X_{1} \rightarrow X_{5}$ and $X_{1} \rightarrow X_{2}$ in the 
lower diagram in Fig.5-11, with the conditions of Park and Stafford: $\hbar \omega_{1}=1.78 \mathrm{eV}, \quad \hbar \omega_{2}=5.43 \mathrm{eV}$, and the laser polarized $32^{\circ}$ from the (100) axis in a [100] plane. The vertical arrows indicate the angles at which $\hat{\varepsilon}_{1}$ and $\hat{\varepsilon}_{2}$ are parallel and perpendicular. We see that the functions have the correct shape, with the maximum for $X_{1} \rightarrow X_{5}$ shifted to the left of $32^{\circ}$ and the $x_{1} \rightarrow x_{2}$ maximum shifted to the right. The data of Park and Stafford in the upper diagram appears to have its maximum slightly to the right of $32^{\circ}$. Thus if the matrix element for $x_{1} \rightarrow x_{2}$ is larger than that for $X_{1} \rightarrow X_{5}$, the polarization dependence would be sinusoidal with the maximum shifted in the same way as that measured by Park and Stafford.

The ratios of maximum to minimum, however, are different, with the experimental value of about 1.5 and the calculated value for $X_{1} \rightarrow X_{2}$ of 1.82 . This could be due to the fact that a forbidden $\mathrm{X}$-point transition can not be the only transition occurring at $7.21 \mathrm{eV}$, and small contributions of other transitions could alter this ratio. It should be noted that a sole addition of $X_{1} \rightarrow X_{5}$ dependence can not sufficiently improve the calculated ratio; the minimum ratio for any linear combination of $X_{1} \rightarrow X_{2}$ and $\mathrm{X}_{1} \rightarrow \mathrm{X}_{5}$ functions is 1.59 .

The possibility of the peak at $7.21 \mathrm{eV}$ occurring near $X$ is given some support by the fact that the energy difference $(\sim 0.15)$ between the peaks at $7.21 \mathrm{eV}$ and 7.375 
is close to the energy difference of points $x_{7}^{-}$and $x_{6}^{-}$ of $0.21 \mathrm{eV}$ calculated by Onodera et al., and it is close to the energy difference between the one-photon peaks at $6.7 \mathrm{eV}$ and $6.9 \mathrm{eV}$ which have been interpreted ${ }^{51}$ as due to s excitons for $x_{7}^{+}, x_{7}^{-}$and $x_{6}^{-}, x_{7}^{+}$. The question now remains of why a peak did not appear in the one-photon. spectrum which would correspond to the $\mathrm{x}_{6}^{-} \rightarrow \mathrm{x}_{7}^{+}$transition. This could result if the one-photon 7.24 peak occurred with $\Gamma_{-}^{8} \rightarrow \Gamma_{+}^{6}$ interband absorption which falls off rapidly at slightly higher energy. A more likely possibility, however, is that the $7.24 \mathrm{eV}$ peak does not have its origin in the $\mathrm{x}_{7}^{-} \rightarrow \mathrm{x}_{7}^{+}$transition, but is indeed due to a $\Gamma_{-}^{6}, \Gamma_{+}^{6} \mathrm{~s}$ exciton, as suggested by Onodera et al..$^{51}$ and Teegarden et al. 66

As mentioned earlier, Park and Stafford could not measure the polarization dependence of the peak at 7.375 $\mathrm{eV}$, but they indicated that they would attempt this after new equipment is prepared. Since $\bar{x}_{6}^{-} \times X_{7}^{+}$contains $x_{5}$ but not $x_{2}$, we can predict that if in fact the peak at $7.375 \mathrm{eV}$ is due to an $\mathrm{X}_{6}^{-} \rightarrow \mathrm{X}_{7}^{+}$forbidden transition, the polarization dependence will have the behavior indicated in Fig.5-10 for $X_{1} \rightarrow X_{5}$, with the maximum shifted to the left of $32^{\circ}$. 


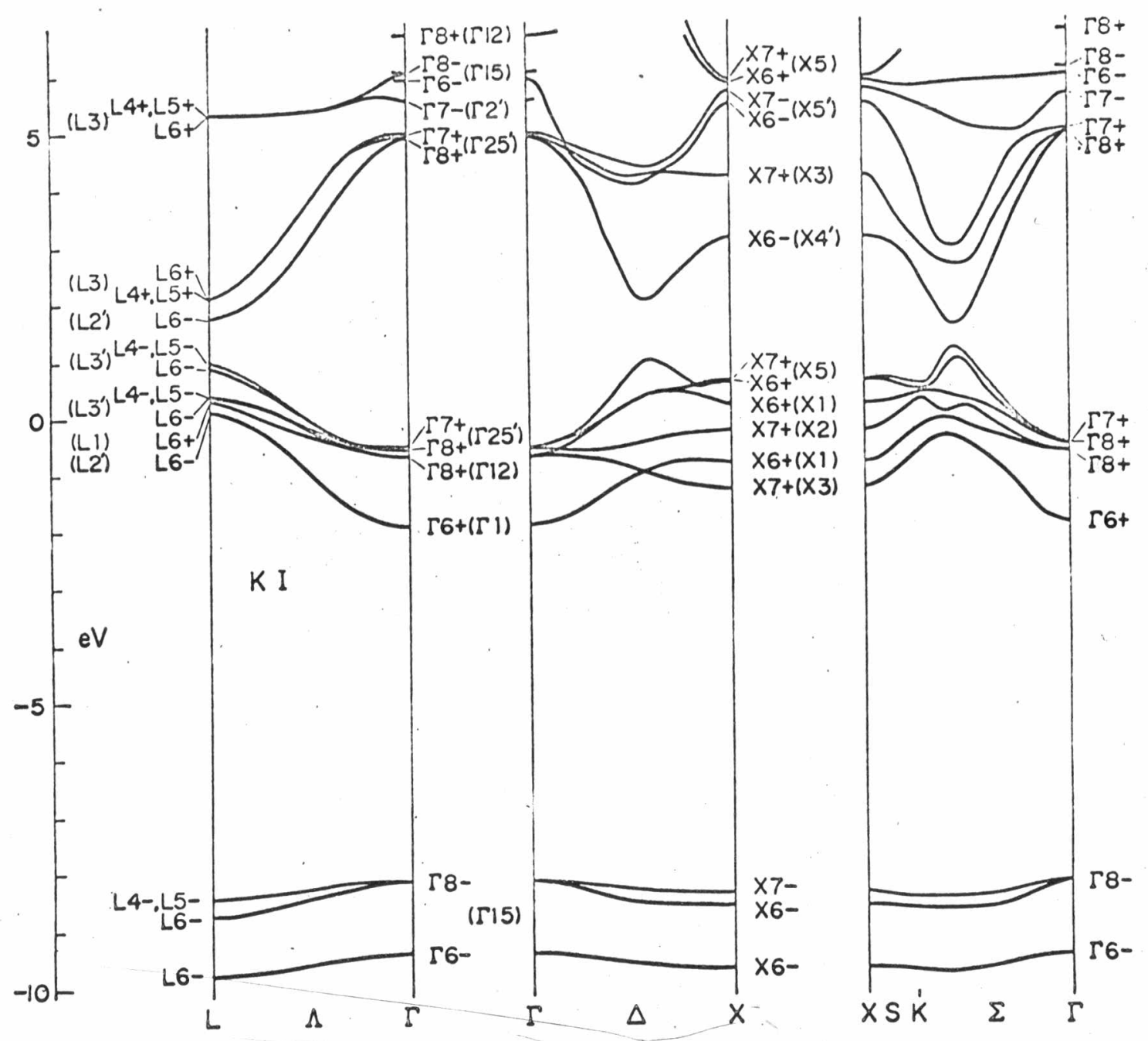

Fig.5-9. Band structure of KI (after Onodera, Okazaki and Inui, Ref. 51) 


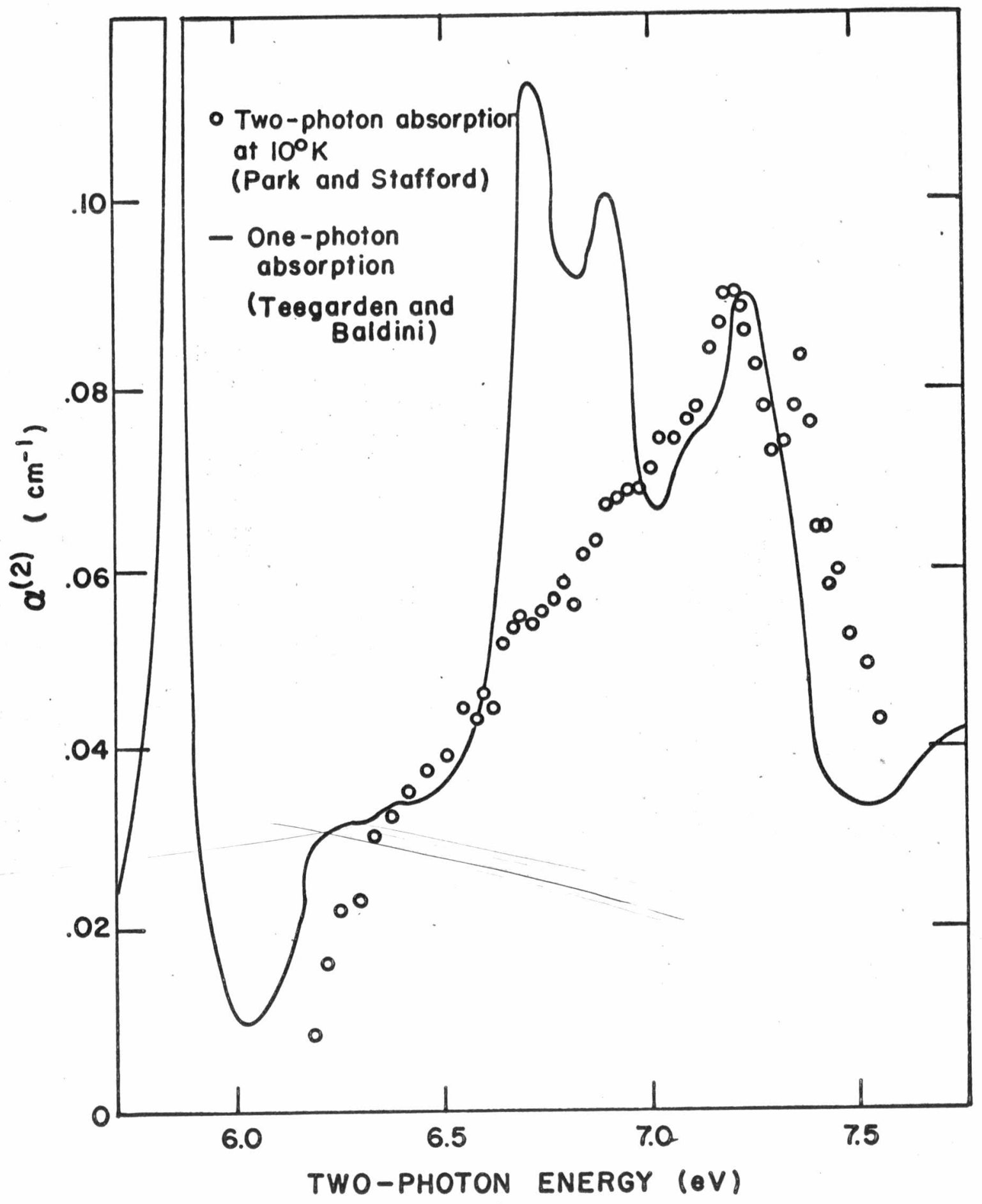

Fig.5-10. Two-photon absorption spectrum of KI (after Park and Stafford, Ref. 16). One-photon spectrum is from Teegarden and Baldinis Ref. 66. 

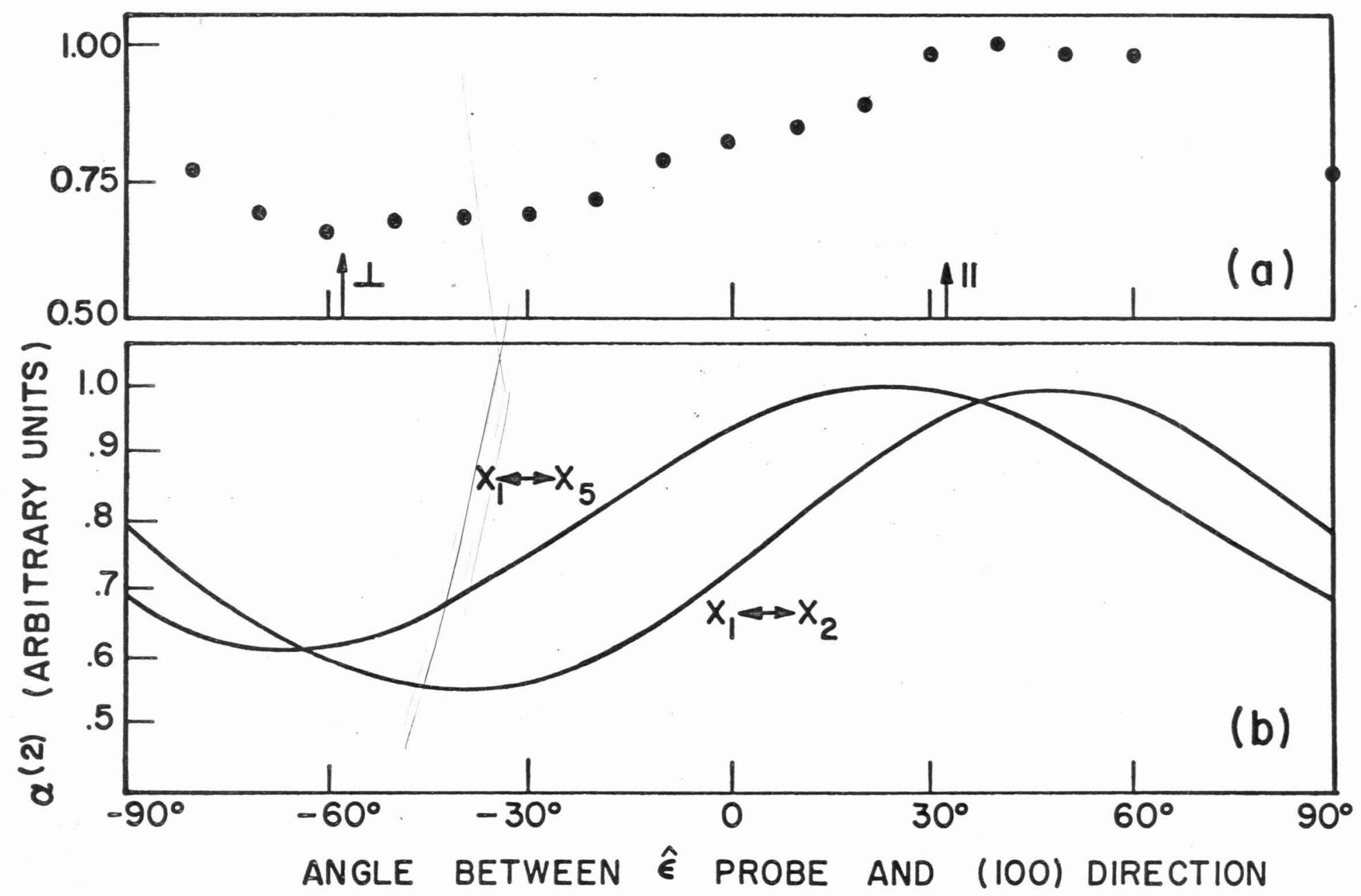

Fig.5-11. (a) Polarization dependence of two-photon absorption in KI (after Park and Stafford, Ref. 16). (b) Theoretical dependence for forbidden X-point transitions. 
F. TICl

Matsuoka ${ }^{14}$ has measured the two-photon absorption spectrum of TlCl near the absorption edge, from about 3.4 $\mathrm{eV}$ to about $3.7 \mathrm{eV}$, and has obtained polarization dependence data at energies ranging from $3.55 \mathrm{eV}$ to $4.33 \mathrm{eV}$. At each of these energies the absorption coefficient was measured as a function of the angle $\theta_{1}$, of the probe light polarization in a (100) plane with respect to a [100] axis for two angles $\theta_{2}$ of the laser polarization: $\theta_{2}=0$ and $\theta_{2}=\frac{\pi}{4}$. Any final state of the crystal must transform according to a representation of $O_{h}$. Therefore the angular dependence for transitions to any possible combination of final states must have the form

$$
\begin{aligned}
\alpha^{(2)}= & a_{1} A_{1 g}\left(\hat{\varepsilon}_{1} \hat{\varepsilon}_{2}\right)+a_{2} E_{g}\left(\hat{\varepsilon}_{1}, \hat{\varepsilon}_{2}\right) \\
& +a_{3} T_{1 g}\left(\hat{\varepsilon}_{1} \hat{\varepsilon}_{2}\right)+a_{4} T_{2 g}\left(\hat{\varepsilon}_{1} \hat{\varepsilon}_{2}\right)
\end{aligned}
$$

where $a_{i}$ is a positive constant and $A_{1 g}\left(\hat{\varepsilon}_{1}, \hat{\varepsilon}_{2}\right)$, etc., are defined in Table 3-1. For Matsuoka!s configurations we find

$$
\begin{aligned}
& \alpha_{\max }^{(2)}(0)=a_{1}+a_{2} \\
& \alpha_{\operatorname{mix}}^{(2)}(0)=a_{3}+a_{4}
\end{aligned}
$$

and

$$
\begin{aligned}
& \alpha_{\max }^{(2)}\left(\frac{\pi}{4}\right)=a_{1}+\frac{1}{4} a_{2}+a_{4} \\
& \alpha_{\min }^{(2)}\left(\frac{\pi}{4}\right)=\frac{3}{4} a_{2}+a_{3} .
\end{aligned}
$$


(The labels indicating maximum and minimum might be interchanged, but this is unimportant here.) Therefore we must have

$$
\alpha_{\max }^{(2)}(0)+\alpha_{\min }^{(2)}(0)=\alpha_{\max }^{(2)}\left(\frac{\pi}{4}\right)+\alpha_{\min }^{(2)}\left(\frac{\pi}{4}\right)
$$

for any possible combination of final states. Matsuoka's data do not conform to this restriction, and hence his polarization measurements can not be explained in terms of any electronic states of the crystal. 


\section{Chapter VI \\ Conclusion}

Angular dependence functions for allowed two-photon absorption have been found for transitions between oneelectron states of arbitrary symmetries for $\Gamma$-point transitions in all crystal point groups. Those obtained for $\Gamma^{\prime} \longrightarrow \Gamma^{\mu}$ transitions agree in most cases with those obtained earlier by Inoue and Toyozawa. ${ }^{11}$ For non-central symmetry points it was shown how to obtain the angular dependence for transitions between states of arbitrary symmetries using the tables of Inoue and Toyozawa for these symmetry points and the tables for the $\Gamma$-point transitions. Angular functions were calculated and tabulated for transitions occurring at symmetry points in hexagonal close-packed and wurtzite structures. The angular functions for allowed transitions are supplementary to those obtained by the earlier workers, and they are intended to aid the experimentalist in determining oneelectron state symmetries responsible for observed twophoton absorption.

The angular dependence for forbidden transitions has been calculated in a simple two band model neglecting exciton effects. In this model the ratio of maximum to 
minimum absorption was found to be a simple function of the energies of the photons participating in the absorption. The earlier calculation of $\operatorname{Mahan}^{20}$ which included exciton effects gave similar results with, however, a more complicated expression for the ratio of maximum to minimum absorption. The neglect of exciton effects is expected to be a good approximation in the continuum region of the absorption due to the small electron-hole pairs. Since band gap energies and exciton binding energies upon which Mahan's maximum to minimum ratio depends are often not known very accurately, the simpler model is expected to give more accurate results for the polarization dependence in the continuum. This contention has been given experimental support in the measurements on $\mathrm{RbI} .12$

The effect of $\vec{k} \cdot \vec{p}$ splitting in the case of $p$-like valence bands has been shown to alter the ratio of maximum to minimum; when substantial splitting occurs in the valence bands this ratio is no longer confined to values between $I$ and 2 , as predicted when $\vec{k} \cdot \vec{p}$ splitting is neglected, and this should be kept in mind when interpreting the polarization dependence.

Since the group of the wave vector at the $X$-point in simple cubic and face-centered cubic structures contains the inversion, forbidden transitions can occur near this point. The energy surfaces about $X$, however, are not in general spherical, so that the previous calculations are not 
applicable. The polarization dependence was calculated for these transitions, and it was found to depend both on the symmetries of the initial and final states and on the orientations of the polarization vectors with respect to the crystal axes. The results were used to suggest that two absorption peaks in $\mathrm{KI}^{16}$ could be due to transitions near $X$. The peak upon which polarization dependence measurements were made showed the correct polarization behavior; the polarization dependence of the second peak, which is separated from the first approximately by the energy difference of two valence bands near $X$, has been predicted on the basis of the symmetries of the valence and conduction bands at this point.

Polarization studies of two-photon absorption in solids very quickly followed the initial theoretical work by Inoue and Toyozawa, and Mahan's extension of the theory to forbidden transitions has given added impetus to the experimental work. The increasing interest in this work reflected in the rising frequency of experimental reports has indicated that further theoretical work has been warranted. It is hoped that the investigations contained herein will be of assistance in the interpretation of two-photon absorption spectra. 


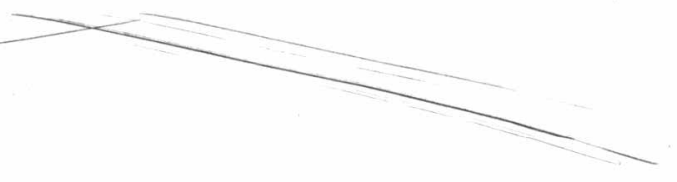

Appendix A

$\vec{k} \cdot \vec{p}$ Perturbation Theory

Bloch functions are solutions to an equation

$$
\left[-\frac{\hbar^{2}}{2 m} \nabla^{2}+V(\vec{r})\right] \psi_{n k}(\vec{r})=E_{n}(k) \psi_{n k}(\vec{r})
$$

where $V(\vec{r})$ is a potential which has the periodicity of the crystal lattice. Bloch's theorem states that one can write

$$
\psi_{n k}(\vec{r})=e^{i \vec{k} \cdot \vec{r}} u_{n k}(\vec{r})
$$

where $u_{n k}(\vec{r})$ has the periodicity of the lattice. If this expression is substituted into Eq.A-I, one obtains

$$
\begin{aligned}
{\left[-\frac{\hbar^{2}}{2 m} \nabla^{2}+V(\vec{r})\right] u_{n k}(\vec{r}) } & +\left[\frac{\hbar}{m} \vec{k} \cdot \vec{p}+\frac{\hbar^{2} k^{2}}{2 m}\right] u_{n k}(\vec{r}) \\
& =E_{n}(k) u_{n k}(\vec{r}) .
\end{aligned}
$$

If we know the solution of this equation at some point, say $\vec{k}=0$ for simplicity, an approximate solution at a nearby point can be obtained by treating the second term on the left as a perturbation. Assume that $\boldsymbol{U}_{n_{0}}(\vec{r})$ is non-degenerate. To second order 21 we have

$$
\begin{aligned}
E_{n}(\vec{k}) & =E_{n}(0)+\frac{\hbar^{2} k^{2}}{2 m}+\frac{\hbar^{2}}{m^{2}} \sum_{l \neq n} \frac{\langle n(0)|\vec{k} \cdot \vec{p}| \ell(0)\rangle\langle l(0)|\vec{k} \cdot \vec{p}| n(0)\rangle}{E_{n}(0)-E_{l}(0)} \\
& =E_{n}(0)+\frac{\hbar^{2}}{2} \vec{k} \cdot\left(\frac{1}{m^{*}}\right) \cdot \vec{k}
\end{aligned}
$$


where the effective mass

$$
\begin{aligned}
\left(\frac{1}{m^{*}}\right)_{\alpha \beta} \equiv \frac{1}{m}+\frac{1}{m^{2}} \sum_{l \neq n} & {\left[\frac{\left\langle n(0)\left|p_{\alpha}\right| l(0)\right\rangle\left\langle l(0)\left|p_{\beta}\right| n(0)\right\rangle}{E_{n}(0)-E_{l}(0)}\right.} \\
& \left.+\frac{\left\langle n(0)\left|p_{\beta}\right| l(0)\right\rangle\left\langle l(0)\left|p_{\alpha}\right| n(0)\right\rangle}{E_{n}(0)-E_{l}(0)}\right]
\end{aligned}
$$

The perturbed function is

$$
u_{n k}(\vec{r}) \cong u_{n_{0}}(\vec{r})+\frac{\hbar}{m} \sum_{l \neq n} \frac{\langle l(0)|\vec{k} \cdot \vec{p}| n(0)\rangle}{E_{n}(0)-E_{l}(0)} u_{l 0}(\vec{r}) \quad(A-3)
$$

When $U_{n k}(\vec{r})$ is degenerate at $\vec{k}=0$ one must diagonalize the matrix

$$
\sum_{l=\left\{n_{i}\right\}} \frac{\left\langle n_{i}(0)|\vec{k} \cdot \vec{p}| l(0)\right\rangle\left\langle\ell(0)|\vec{k} \cdot \vec{p}| n_{j}(0)\right\rangle}{E_{n}(0)-E_{l}(0)}
$$

The solution of the associated secular equation enables one to find the correct linear combinations $U_{s o}(\vec{r})$ of $u_{n_{i} \circ}(\vec{r})$ such that the energy of band $s$ is given by

$$
E_{s}(k)=E_{s}(0)+\frac{\hbar^{2}}{2} \vec{k} \cdot\left(\frac{\overleftrightarrow{1}}{m_{s}^{*}}\right) \cdot \vec{k}
$$

and the perturbed functions by

$$
u_{s k}(\vec{r}) \cong u_{s 0}(\vec{r})+\frac{\hbar}{m} \sum_{l \neq\{s)} \frac{\langle l(0)|\vec{k} \cdot \vec{p}| s(0)\rangle}{E_{s}(0)-E_{l}(0)} u_{l 0}(\vec{r}) .
$$

In general $\left(\overrightarrow{1 / m_{s}^{*}}\right)$ is a function of $\hat{k}$ for bands which are degenerate at $\vec{k}=0$. For bands which remain degenerate near $\vec{k}=0$, the matrix diagonalization is not necessary and the effective masses for these bands are all equal. 


\section{Appendix B \\ Symmetry of $\bigwedge(\omega)$}

Let us first consider the sum over intermediate states ignoring exciton effects. Let $\left|n\left(k_{0}\right)\right\rangle=|\nu m, a\rangle$, where $m$ is the row of representation $\nu$, and a labels all other quantum numbers.

$$
\Lambda(\omega)=\sum_{a \nu m} \frac{|\nu m, a\rangle\langle\nu m, a|}{E(\nu, a)-\hbar \omega}
$$

Operate on this by an element $P_{R}$ of the group of $\vec{k}_{0}$ :

$$
\begin{aligned}
P_{R} \Lambda(\omega) P_{R}^{-1} & =\sum_{a \nu m} \frac{P_{R}|\nu m, a\rangle\langle\nu m, a| P_{R}^{-1}}{E(\nu, a)-\hbar \omega} \\
& =\sum_{a \nu m} \sum_{n s} \Gamma_{n m}^{\nu}(R) \Gamma_{m s}^{\nu}(R) \frac{|\nu n, a\rangle\langle\nu s, a|}{E(\nu, a)-\hbar \omega} \\
& =\sum_{a \nu n s} \Gamma_{n s}^{\nu}\left(R R^{-1}\right) \frac{|\nu n, a\rangle\langle\nu s, a|}{E(\nu, a)-\hbar \omega} \\
& =\sum_{a \nu n s} \delta_{n s} \frac{|\nu n, a\rangle\langle\nu s, a|}{E(\nu, a)-\hbar \omega} \\
& =\sum_{a \nu n} \frac{|\nu n, a\rangle\langle\nu n, a|}{E(\nu, a)-\hbar \omega}
\end{aligned}
$$

When excitons are included in the intermediate states the proof is exactly the same because of our neglect of the dependence of 


$$
N^{\frac{1}{2}} \sum_{\nu \beta} \frac{U_{c_{i} v_{j}}^{\mu}(\vec{\beta})^{*} U_{n_{j}^{+} v_{j}}^{\nu}(\vec{\beta}) U_{n_{l}^{+} v_{j}}^{\nu}(0)^{*}}{E_{n_{l}^{+} v_{j}}(p)-\hbar \omega}
$$

and the similar expression with $n_{\vec{l}}$, on the index $l_{\text {. }}$ 


\section{Appendix C}

Derivation of the Clebsch-Gordan Symmetry Relation Since* the Clebsch-Gordan coefficients are elements of a unitary matrix they obey the orthogonality relations

and

$$
\begin{aligned}
& \sum_{j \ell}\left[\begin{array}{ccc}
\lambda \tau_{\lambda}, \mu \nu \\
s, l j
\end{array}\right]^{*}\left[\begin{array}{ccc}
\lambda^{\prime} \tau_{\lambda^{\prime}}^{\prime}, \mu \nu \\
s^{\prime}, & l j
\end{array}\right]=\delta\left(\lambda, \lambda^{\prime}\right) \delta\left(\tau_{\lambda}, \tau_{\lambda^{\prime}}^{\prime}\right) \delta\left(s, s^{\prime}\right) \\
& \sum_{\lambda \tau_{\lambda} s}\left[\begin{array}{cc}
A^{\lambda \tau_{\lambda},}, \mu \nu \\
s, l_{j}
\end{array}\right]^{*}\left[\begin{array}{cc}
\lambda \tau_{\lambda}, & \mu \nu \\
s, & l^{\prime} j^{\prime}
\end{array}\right]=\delta\left(l, \ell^{\prime}\right) \delta\left(j, j^{\prime}\right) \text {. }
\end{aligned}
$$

From the definition of the Clebsch-Gordan coefficients we can write

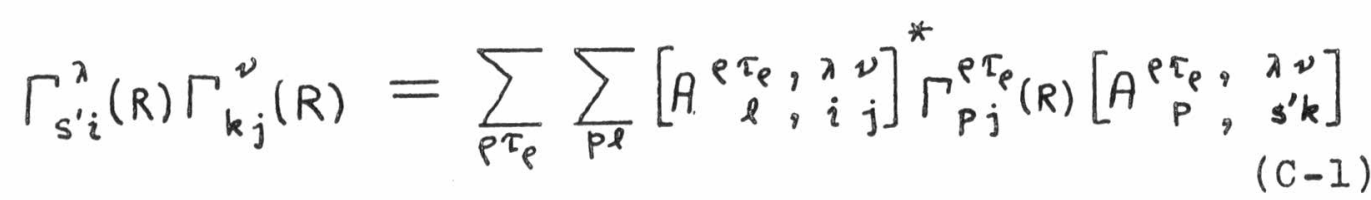

and

$$
\Gamma_{m l}^{\mu}(R) \Gamma_{k j^{\prime}}^{v}(R)=\sum_{\gamma \tau_{\gamma}} \sum_{s s^{\prime}}\left[\begin{array}{cc}
A^{\gamma \tau_{\gamma}} & \mu \nu \\
s, & l_{j^{\prime}}
\end{array}\right]^{*} \Gamma_{s^{\prime} s}^{\gamma \tau_{\gamma}}(R)\left[\begin{array}{cc}
A^{\gamma \tau_{\gamma}}, & \mu \nu \\
s^{\prime}, & m k \\
(C-2) & .
\end{array}\right.
$$

Multiply Eq.C-2 by $\Gamma_{k j}^{\nu}(R)^{*}$ and sum over $k$ to obtain

$$
\Gamma_{m l}^{\mu}(R) \delta\left(j, j^{\prime}\right)=\sum_{\gamma \tau_{\gamma}} \sum_{s s^{\prime} k}\left[\begin{array}{cc}
A^{\gamma \tau_{\gamma},} & \mu \nu \\
s, & j^{\prime}
\end{array}\right]^{*} \Gamma_{s^{\prime} s}^{\gamma}(R) \Gamma_{k j}^{\nu}(R)^{*}\left[\begin{array}{cc}
\gamma \tau_{\gamma}, \mu \nu \\
s^{\prime}, & m k
\end{array}\right],
$$

where it has been observed that $\Gamma_{s^{\prime} s}^{\gamma \tau_{\gamma}}(R)=\Gamma_{s^{\prime} s}^{\gamma}(R)$.

Multiplying this by $A_{i}^{\lambda} \tau_{i}, l^{\mu \nu}$, and summing over $I$ and $j^{\prime}$ yields 
154

$$
\sum_{l}\left[\begin{array}{cc}
\lambda \tau_{\lambda}, & \mu \nu \\
i, & l j
\end{array}\right] \Gamma_{m \ell}^{\mu}(R)=\sum_{s^{\prime} k} \Gamma_{s^{\prime} i}^{\lambda}(R) \Gamma_{k j}^{\nu}(R)^{*}\left[\begin{array}{cc}
\lambda \tau_{\lambda}, & \mu \nu \\
s^{\prime}, & m k
\end{array}\right]_{(C-3)}
$$

Change $p$ to $\bar{v}$ in Eq.C-I and substitute into Eq.C-3 to obtain

$$
\begin{aligned}
& \sum_{\ell}\left[\begin{array}{ccc}
\lambda \tau_{\lambda}, & \mu \nu \\
i & , l j
\end{array}\right] \Gamma_{m l}^{\mu}(R) \\
& =\sum_{\rho \tau_{\rho} p} \sum_{l s^{\prime} k}\left[\begin{array}{cc}
A^{\rho \tau_{\rho}}, \lambda \bar{p} \\
l, & i j
\end{array}\right]^{*} \Gamma_{\rho l}^{\rho}(R)\left[\begin{array}{ccc}
\rho \tau_{\rho}, & \lambda \bar{p} \\
p, & s^{\prime} k
\end{array}\right]\left[\begin{array}{cc}
A^{\lambda \tau_{\lambda},} & \mu \nu \\
s^{\prime}, & m k
\end{array}\right]
\end{aligned}
$$

Multiply by $A \begin{gathered}\sigma_{\tau_{\sigma}}, i_{i} \bar{\nu} \\ j\end{gathered}$ and sum over $i$ and $j:$

$$
\begin{aligned}
& \sum_{\ell i j} \Gamma_{m l}^{\mu}(R)\left[\begin{array}{cc}
\lambda \tau_{\lambda}, & \mu \nu \\
i & l j
\end{array}\right]\left[\begin{array}{cc}
\sigma \tau_{\sigma}, \lambda i \\
n, & i j
\end{array}\right] \\
& =\sum_{p s^{\prime} k}\left[\begin{array}{cc}
\sigma \tau_{\sigma}, & \lambda \bar{\nu} \\
P, & s^{\prime} k
\end{array}\right]\left[\begin{array}{cc}
\lambda \tau_{\lambda}, \mu \nu \\
s^{\prime}, & m k
\end{array}\right] \Gamma_{p^{n}}^{\sigma}(R) .
\end{aligned}
$$

This can be written

$$
\sum_{l} \Gamma_{m l}^{\mu}(R) M_{l n}=\sum_{p} M_{m p} \Gamma_{p n}^{\sigma}(R),
$$

where

$$
M_{l n}=\sum_{i j}\left[\begin{array}{cc}
\lambda \tau_{\lambda}, \mu \nu \\
i, & l j
\end{array}\right]\left[\begin{array}{cc}
\sigma \tau_{\sigma}, \lambda \bar{\nu} \\
n, & i j
\end{array}\right],
$$

or, in matrix form,

$$
\Gamma^{\mu}(R) M=M \Gamma^{\mu}(R)
$$

If $\Gamma^{\mu}$ and $\Gamma^{\sigma}$ are inequivalent then Schur's lemma says that $M$ is the null matrix. If $\Gamma^{\mu}$ is equivalent to $\Gamma^{\sigma}$, then $M$ is a constant multiple of the unit matrix: 


$$
\sum_{i j}\left|A_{i, l_{j} \tau_{\lambda}, \mu \nu}\right|\left|A \begin{array}{c}
\sigma \tau_{\sigma}, \lambda \bar{\nu} \\
i, i_{j}
\end{array}\right|=C\left[(\lambda \mu) \bar{\nu} ; \tau_{\lambda} \tau_{\mu}\right] \delta(\sigma, \mu) \delta(n, l),
$$

where the parentheses in the constant $C$ indicate the pairs of indices which are interchanged on the Clebsch-Gordan coefficients. Bringing the second factor on the left side to the right we have the result

$$
\left[\begin{array}{cc}
\lambda \tau_{\lambda}, \mu \nu \\
i, n_{j}
\end{array}\right]=\sum_{\tau_{\mu}} C\left[(\lambda \mu) \bar{\nu} ; \tau_{\lambda} \tau_{\mu}\right]\left[\begin{array}{cc}
\mu \tau_{\mu}, & \lambda \nu \\
n & i j
\end{array}\right]^{*}
$$

*This follows the treatment given by M. Hamermesh Group Theory (Addison-Wesley Publishing Co., Reading, Mass., 1962), p. 260 for real representations in his discussion of the symmetric group. 


\section{Appendix D}

Table D-1: Correspondence of present notation with that of Koster et al. (Ref. 42 ) for the single-valued representations.

\begin{tabular}{l||c|c|c|c|c|c|c|c|c|c|c|c|} 
Group & $\mathrm{C}_{1}$ & $\mathrm{~S}_{2}$ & \multicolumn{2}{|c|}{$\mathrm{C}_{2}$} & \multicolumn{3}{|c|}{$\mathrm{C}_{1 \mathrm{~h}}$} & \multicolumn{3}{c|}{$\mathrm{C}_{2 \mathrm{~h}}$} & \multicolumn{4}{|c|}{$\mathrm{D}_{2}$} \\
\hline $\begin{array}{l}\text { Present } \\
\text { Notation }\end{array}$ & $\mathrm{A}$ & $\mathrm{A}_{ \pm}$ & $\mathrm{A}$ & $\mathrm{B}$ & $\mathrm{A}^{\prime}$ & $\mathrm{A}^{\prime \prime}$ & $\mathrm{A}_{ \pm}$ & $\mathrm{B}_{ \pm}$ & $\mathrm{A}$ & $\mathrm{B}_{1}$ & $\mathrm{~B}_{2}$ & $\mathrm{~B}_{3}$ \\
\hline $\begin{array}{l}\text { Notation of } \\
\text { Ref. }\end{array}$ & $\Gamma_{1}$ & $\Gamma_{1}^{ \pm}$ & $\Gamma_{1}$ & $\Gamma_{2}$ & $\Gamma_{1}$ & $\Gamma_{2}$ & $\Gamma_{1}^{ \pm}$ & $\Gamma_{2}^{ \pm}$ & $\Gamma_{1}$ & $\Gamma_{3}$ & $\Gamma_{2}$ & $\Gamma_{4}$ \\
\hline
\end{tabular}

\begin{tabular}{|c|c|c|c|c|c|c|c|c|c|c|c|c|c|}
\hline \multicolumn{4}{|c|}{$\mathrm{C}_{2 \mathrm{v}}$} & \multicolumn{4}{|c|}{$\mathrm{D}_{2 \mathrm{~h}}$} & \multicolumn{3}{|c|}{$\mathrm{C}_{4}$} & \multicolumn{3}{|c|}{$\mathrm{S}_{4}$} \\
\hline $\mathrm{A}_{1}$ & $A_{2}$ & $\mathrm{~B}_{1}$ & $\mathrm{~B}_{2}$ & $A_{ \pm}$ & $B_{1 \pm}$ & $\mathrm{B}_{2 \pm}$ & $\mathrm{B}_{3 \pm}$ & A & B & $E$ & A & $B$ & $E$ \\
\hline$\Gamma_{1}$ & $\Gamma_{3}$ & $\Gamma_{2}$ & $\Gamma_{4}$ & $\Gamma_{1}^{ \pm}$ & $\Gamma_{3}^{ \pm}$ & $\Gamma_{2}^{ \pm}$ & $\Gamma_{4}^{ \pm}$ & $\Gamma_{1}$ & $\Gamma_{2}$ & $\left(\begin{array}{ll}\Gamma_{3} & \Gamma_{4}\end{array}\right)$ & $\Gamma_{1}$ & $\Gamma_{2}$ & $\left(\Gamma_{3} \Gamma_{4}\right)$ \\
\hline
\end{tabular}

\begin{tabular}{|c|c|c|c|c|c|c|c|c|c|c|c|c|c|c|c|c|c|}
\multicolumn{10}{c|}{$\mathrm{C}_{4 \mathrm{~h}}$} & \multicolumn{10}{c|}{$\mathrm{C}_{4 \mathrm{v}}$} & \multicolumn{10}{c|}{$\mathrm{D}_{2 \mathrm{~d}}$} \\
\hline $\mathrm{A}_{ \pm}$ & $\mathrm{B}_{ \pm}$ & $\mathrm{E}_{ \pm}$ & $\mathrm{A}_{1}$ & $\mathrm{~A}_{2}$ & $\mathrm{~B}_{1}$ & $\mathrm{~B}_{2}$ & $\mathrm{E}$ & $\mathrm{A}_{1}$ & $\mathrm{~A}_{2}$ & $\mathrm{~B}_{1}$ & $\mathrm{~B}_{2}$ & $\mathrm{E}$ & $\mathrm{A}_{1}$ & $\mathrm{~A}_{2}$ & $\mathrm{~B}_{1}$ & $\mathrm{~B}_{2}$ & $\mathrm{E}$ \\
\hline$\Gamma_{1}^{ \pm}$ & $\Gamma_{2}^{ \pm}$ & $\left(\Gamma_{3}^{ \pm} \Gamma_{4}^{ \pm}\right)$ & $\Gamma_{1}$ & $\Gamma_{2}$ & $\Gamma_{3}$ & $\Gamma_{4}$ & $\Gamma_{5}$ & $\Gamma_{1}$ & $\Gamma_{2}$ & $\Gamma_{3}$ & $\Gamma_{4}$ & $\Gamma_{5}$ & $\Gamma_{1}$ & $\Gamma_{2}$ & $\Gamma_{3}$ & $\Gamma_{4}$ & $\Gamma_{5}$ \\
\hline
\end{tabular}

\begin{tabular}{|c|c|c|c|c|c|c|c|c|c|c|c|}
\multicolumn{9}{|c|}{$\mathrm{D}_{4 \mathrm{~h}}$} & \multicolumn{2}{|c|}{$\mathrm{C}_{3}$} & \multicolumn{2}{|c|}{$\mathrm{S}_{6}$} & \multicolumn{3}{c|}{$\mathrm{D}_{3}$} \\
\hline $\mathrm{A}_{1 \pm}$ & $\mathrm{A}_{2 \pm}$ & $\mathrm{B}_{1 \pm}$ & $\mathrm{B}_{2 \pm}$ & $\mathrm{E}_{ \pm}$ & $\mathrm{A}$ & $\mathrm{E}$ & $\mathrm{A}_{ \pm}$ & $\mathrm{E}_{ \pm}$ & $\mathrm{A}_{1}$ & $\mathrm{~A}_{2}$ & $\mathrm{E}$ \\
\hline$\Gamma_{1}^{ \pm}$ & $\Gamma_{2}^{ \pm}$ & $\Gamma_{3}^{ \pm}$ & $\Gamma_{4}^{ \pm}$ & $\Gamma_{5}^{ \pm}$ & $\Gamma_{1}$ & $\left(\Gamma_{2} \Gamma_{3}\right)$ & $\Gamma_{1}^{ \pm}$ & $\left(\Gamma_{2}^{ \pm} \Gamma_{3}^{ \pm}\right)$ & $\Gamma_{1}$ & $\Gamma_{2}$ & $\Gamma_{3}$ \\
\hline
\end{tabular}


Table D-I, continued:

\begin{tabular}{|c|c|c|c|c|c|c|c|c|c|c|c|c|c|}
\hline \multicolumn{3}{|c|}{$\mathrm{C}_{3 \mathrm{v}}$} & \multicolumn{3}{|c|}{$D_{3 d}$} & \multicolumn{4}{|c|}{$\mathrm{C}_{6}$} & \multicolumn{4}{|c|}{$\mathrm{c}_{3 \mathrm{~h}}$} \\
\hline$A_{1}$ & $\mathrm{~A}_{2}$ & $\mathrm{E}$ & $A_{1 \pm}$ & $\mathrm{A}_{2} \pm$ & $\mathrm{E}_{ \pm}$ & A & B & $E^{\prime}$ & E" & $A^{\prime}$ & $\mathrm{A}^{\prime \prime}$ & $E^{\prime}$ & $\mathrm{E}^{\prime \prime}$ \\
\hline$\Gamma_{1}$ & $\Gamma_{2}$ & $\Gamma_{3}$ & $\Gamma_{1}^{ \pm}$ & $\Gamma_{2}^{ \pm}$ & $\Gamma_{3}^{ \pm}$ & $\Gamma_{1}$ & $\Gamma_{4}$ & $\left(\begin{array}{ll}\Gamma_{5} & \Gamma_{6}\end{array}\right)$ & $\left.\begin{array}{ll}\left(\Gamma_{2}\right. & \Gamma_{3}\end{array}\right)$ & $\Gamma_{1}$ & $\Gamma_{4}$ & $\left(\Gamma_{2} \Gamma_{3}\right)$ & $\left(\Gamma_{5} \Gamma_{6}\right)$ \\
\hline
\end{tabular}

\begin{tabular}{|c|c|c|c|c|c|c|c|c|c|c|c|c|c|c|c|}
\hline \multicolumn{10}{c|}{$\mathrm{C}_{6 \mathrm{~h}}$} & \multicolumn{10}{c|}{$\mathrm{D}_{6}$} \\
\hline $\mathrm{A}_{ \pm}$ & $\mathrm{B}_{ \pm}$ & $\mathrm{E}_{ \pm}^{\prime}$ & $\mathrm{E}_{ \pm}^{\prime \prime}$ & $\mathrm{A}_{1}$ & $\mathrm{~A}_{2}$ & $\mathrm{~B}_{1}$ & $\mathrm{~B}_{2}$ & $\mathrm{E}_{1}$ & $\mathrm{E}_{2}$ & $\mathrm{~A}_{1}$ & $\mathrm{~A}_{2}$ & $\mathrm{~B}_{1}$ & $\mathrm{~B}_{2}$ & $\mathrm{E}_{1}$ & $\mathrm{E}_{2}$ \\
\hline$\Gamma_{1}^{ \pm}$ & $\Gamma_{4}^{ \pm}$ & $\left(\Gamma_{5}^{ \pm} \Gamma_{6}^{ \pm}\right)$ & $\left(\Gamma_{2}^{ \pm} \Gamma_{3}^{ \pm}\right)$ & $\Gamma_{1}$ & $\Gamma_{2}$ & $\Gamma_{3}$ & $\Gamma_{4}$ & $\Gamma_{5}$ & $\Gamma_{6}$ & $\Gamma_{1}$ & $\Gamma_{2}$ & $\Gamma_{4}$ & $\Gamma_{3}$ & $\Gamma_{5}$ & $\Gamma_{6}$ \\
\hline
\end{tabular}

\begin{tabular}{|c|c|c|c|c|c|c|c|c|c|c|c|c|c|c|}
\hline \multicolumn{10}{c|}{$\mathrm{D}_{3 \mathrm{~h}}$} & \multicolumn{1}{c|}{$\mathrm{D}_{6 \mathrm{~h}}$} & \multicolumn{3}{|c|}{$\mathrm{T}$} \\
\hline $\mathrm{A}_{1}$ & $\mathrm{~A}_{2}^{\prime}$ & $\mathrm{A}_{1}^{\prime \prime}$ & $\mathrm{A}_{2}^{\prime \prime}$ & $\mathrm{E}^{\prime}$ & $\mathrm{E}^{\prime \prime}$ & $\mathrm{A}_{1 \pm}$ & $\mathrm{A}_{2 \pm}$ & $\mathrm{B}_{1 \pm}$ & $\mathrm{B}_{2 \pm}$ & $\mathrm{E}_{1 \pm}$ & $\mathrm{E}_{2 \pm}$ & $\mathrm{A}$ & $\mathrm{E}$ & $\mathrm{T}$ \\
\hline$\Gamma_{1}$ & $\Gamma_{2}$ & $\Gamma_{3}$ & $\Gamma_{4}$ & $\Gamma_{6}$ & $\Gamma_{5}$ & $\Gamma_{1}^{ \pm}$ & $\Gamma_{2}^{ \pm}$ & $\Gamma_{3}^{ \pm}$ & $\Gamma_{4}^{ \pm}$ & $\Gamma_{5}^{ \pm}$ & $\Gamma_{6}^{ \pm}$ & $\Gamma_{1}$ & $\left(\Gamma_{2} \Gamma_{3}\right)$ & $\Gamma_{4}$ \\
\hline
\end{tabular}

\begin{tabular}{|l|c|c|c|c|c|c|c|c|c|c|c|c|c|c|c|}
\multicolumn{9}{|c|}{$\mathrm{T}$} & \multicolumn{10}{c|}{$\mathrm{T}_{\mathrm{h}}$} & \multicolumn{10}{|c|}{$\mathrm{T}$} & $\mathrm{T}_{\mathrm{d}}$ \\
\hline $\mathrm{A}$ & $\mathrm{E}$ & $\mathrm{T}$ & $\mathrm{A}_{ \pm}$ & $\mathrm{E}_{ \pm}$ & $\mathrm{T}_{ \pm}$ & $\mathrm{A}_{1}$ & $\mathrm{~A}_{2}$ & $\mathrm{E}$ & $\mathrm{T}_{1}$ & $\mathrm{~T}_{2}$ & $\mathrm{~A}_{1}$ & $\mathrm{~A}_{2}$ & $\mathrm{E}$ & $\mathrm{T}_{1}$ & $\mathrm{~T}_{2}$ \\
\hline$\Gamma_{1}$ & $\left(\Gamma_{2} \Gamma_{3}\right)$ & $\Gamma_{4}$ & $\Gamma_{1}^{ \pm}$ & $\left(\Gamma_{2}^{ \pm} \Gamma_{3}^{ \pm}\right)$ & $\Gamma_{4}^{ \pm}$ & $\Gamma_{1}$ & $\Gamma_{2}$ & $\Gamma_{3}$ & $\Gamma_{4}$ & $\Gamma_{5}$ & $\Gamma_{1}$ & $\Gamma_{2}$ & $\Gamma_{3}$ & $\Gamma_{4}$ & $\Gamma_{5}$ \\
\hline
\end{tabular}

\begin{tabular}{|c|c|c|c|c|}
\multicolumn{6}{|c|}{$\mathrm{O}_{\mathrm{h}}$} \\
\hline $\mathrm{A}_{1 \pm}$ & $\mathrm{A}_{2 \pm}$ & $\mathrm{E}_{ \pm}$ & $\mathrm{T}_{1 \pm}$ & $\mathrm{T}_{2 \pm}$ \\
\hline$\Gamma_{1}^{ \pm}$ & $\Gamma_{2}^{ \pm}$ & $\Gamma_{3}^{ \pm}$ & $\Gamma_{4}^{ \pm}$ & $\Gamma_{5}^{ \pm}$ \\
\hline
\end{tabular}


Appendix E

Table E-1: Angular dependence functions for face-centered cubic (fcc), diamond, zinc blende and simple cubic (sc) structures (after Inoue and Toyozawa, Ref. 11). A, E, T, and $T$ are the angular functions defined in Table $3-1$ for $0, \mathrm{~T}_{\mathrm{d}}$ and $\mathrm{O}_{\mathrm{h}}$.

\begin{tabular}{|c|c|c|c|c|c|}
\hline $\begin{array}{c}f c c \\
\text { diamond }\end{array}$ & $\begin{array}{l}\text { zinc } \\
\text { blende } \\
\triangle\end{array}$ & $\mathrm{sc}$ & $\begin{array}{l}\text { fcc } \\
\text { diamond }\end{array}$ & $\begin{array}{l}\text { zinc } \\
\text { blende }\end{array}$ & sc \\
\hline $\mathrm{A}_{1}$ & $A_{1}$ & $\mathrm{~A}_{1}$ & $\left|\lambda_{1}+2\right|^{2} A_{1}+2\left|\lambda_{1}-1\right|^{2} E$ & $\begin{array}{c}\left|1+\lambda_{1}+\lambda_{2}\right|^{2} A_{1}+\left\{\left|1-\lambda_{1}\right|^{2}\right. \\
\left.+\left|1-\lambda_{2}\right|^{2}+\left|\lambda_{1}-\lambda_{2}\right|^{2}\right\}^{E}\end{array}$ & $\left|\lambda_{1}+2\right|^{2} A+2\left|\lambda_{1}-1\right|^{2} E$ \\
\hline $\mathrm{A}_{2}$ & $\mathrm{~A}_{2}$ & $\mathrm{~A}_{2}$ & $\mathrm{~T}_{1}$ & $\left|\lambda_{3}\right|^{2} T_{1}+T_{2}$ & $\mathrm{~T}_{1}$ \\
\hline $\mathrm{B}_{1}$ & $\mathrm{~B}_{1}$ & $\mathrm{~B}_{1}$ & $\mathrm{E}$ & $\left|\lambda_{4}\right|^{2} T_{1}+T_{2}$ & $\mathrm{E}$ \\
\hline $\mathrm{B}_{2}$ & $\mathrm{~B}_{2}$ & $\mathrm{~B}_{2}$ & & $\left|\lambda_{5}\right|^{2} T_{1}+T_{2}$ & $\mathrm{~T}_{2}$ \\
\hline$E$ & & $E$ & $\left|\lambda_{2}\right|^{2} T_{1}+T_{2}$ & & $\left|\lambda_{2}\right|^{2} T_{1}+T_{2}$ \\
\hline
\end{tabular}

\begin{tabular}{|c|c|c|c|c|c|}
\hline${ }^{A}{ }_{1 g}$ & $\mathrm{~A}_{1}$ & ${ }^{A_{18}}$ & $\left|\lambda_{1}+2\right|^{2} A_{1}+2\left|\lambda_{1}-1\right|^{2} E$ & $\left|\lambda_{1}+2\right|^{2} A_{1}+2\left|\lambda_{1}-1\right|^{2} E$ & $\left|\lambda_{1}+2\right|^{2} A_{1}+2\left|\lambda_{1}-1\right|^{2} E$ \\
\hline$A_{2 g}$ & $\mathrm{~A}_{2}$ & $A_{2 g}$ & $T_{1}$ & $\mathrm{~T}_{1}$ & $\mathrm{~T}_{1}$ \\
\hline $\mathrm{B}_{I g}$ & $\mathrm{~B}_{1}$ & $\mathrm{~B}_{1 g}$ & $E$ & $\mathrm{E}$ & $E_{2}$ \\
\hline $\mathrm{B}_{2 \mathrm{~g}}$ & $\mathrm{~B}_{2}$ & $\mathrm{~B}_{2 \mathrm{~g}}$ & $\mathrm{~T}_{2}$ & $\mathrm{~T}_{2}$ & $\mathrm{~T}_{2}$ \\
\hline$E_{g}$ & $E$ & $E_{g}$ & $\left|\lambda_{2}\right|^{2} \mathrm{~T}_{1}+\mathrm{T}_{2}$ & $\left|\lambda_{2}\right|^{2} T_{1}+T_{2}$ & $\left|\lambda_{2}\right|^{2} T_{1}+T_{2}$ \\
\hline
\end{tabular}


Table E-I (Contd.)

\begin{tabular}{|c|c|c|c|c|c|}
\hline & $\begin{array}{l}\mathrm{A}_{1} \\
\mathrm{~A}_{2} \\
\mathrm{E}\end{array}$ & & $\begin{array}{l}4\left|1-\lambda_{1}\right|^{2} T_{2}+\left|2+\lambda_{1}\right|^{2} A_{1} \\
T_{1} \\
\frac{2}{3}\left|\sqrt{2}+\lambda_{3}\right|^{2} E \\
+\frac{1}{3}\left|\sqrt{2} \lambda_{3}-I\right|^{2} T_{2}+\left|\lambda_{4}\right|^{2} T_{1}\end{array}$ & $\begin{array}{l}4\left|I-\lambda_{1}\right|^{2} T_{2}+\left|2+\lambda_{1}\right|^{2} A_{1} \\
T_{1} \\
\frac{2}{3}\left|\sqrt{2}+\lambda_{3}\right|^{2} \mathrm{E} \\
+\frac{1}{3}\left|\sqrt{2} \lambda_{3}-I\right|^{2} T_{2}+\left|\lambda_{4}\right|^{2} T_{1}\end{array}$ & $\begin{array}{l}4\left|1-\lambda_{1}\right|^{2} T_{2}+\left|2+\lambda_{1}\right|^{2} A_{1} \\
T_{1} \\
\frac{2}{3}\left|\sqrt{2}+\lambda_{3}\right|^{2} T_{1} \\
+\frac{1}{3}\left|\sqrt{2} \lambda_{3}-1\right|^{2} T_{2}+\left|\lambda_{4}\right|^{2} T_{1}\end{array}$ \\
\hline \multicolumn{2}{|c|}{ I } & $\mathrm{R}$ & & & \\
\hline $\begin{array}{l}\mathrm{A}_{1 \mathrm{~g}} \\
\mathrm{~A}_{2 \mathrm{~g}} \\
\mathrm{E}_{\mathrm{g}}\end{array}$ & $\begin{array}{l}\mathrm{A}_{1} \\
\mathrm{~A}_{2} \\
\mathrm{E}\end{array}$ & $\begin{array}{l}\mathrm{A}_{1 g} \\
\mathrm{E}_{\mathrm{g}} \\
\mathrm{T}_{1 \mathrm{~g}} \\
\mathrm{~T}_{2 \mathrm{~g}}\end{array}$ & $\begin{array}{l}4\left|1-\lambda_{1}\right|^{2} T_{2}+\left|2+\lambda_{1}\right|^{2} A_{1} \\
T_{1} \\
\frac{2}{3}\left|\sqrt{2}+\lambda_{3}\right|^{2} \mathrm{E} \\
+\frac{1}{3}\left|\sqrt{2} \lambda_{3}-1\right|^{2} T_{2}+\left|\lambda_{4}\right|^{2} T_{1}\end{array}$ & $\begin{array}{l}4\left|1-\lambda_{1}\right|^{2} T_{2}+\left|2+\lambda_{1}\right|^{2} A_{1} \\
T_{1} \\
\frac{2}{3}\left|\sqrt{2}+\lambda_{3}\right|^{2} \mid E \\
+\frac{1}{3}\left|\sqrt{2} \lambda_{3}-1\right|^{2} T_{2}+\left|\lambda_{4}\right|^{2} T_{1}\end{array}$ & $\begin{array}{l}\mathrm{A}_{1} \\
\mathrm{E} \\
\mathrm{T}_{1} \\
\mathrm{~T}_{2}\end{array}$ \\
\hline
\end{tabular}


Table E-I (Contd.)

\begin{tabular}{|c|c|c|c|c|c|}
\hline \multicolumn{3}{|c|}{$\Sigma$} & & & \\
\hline $\mathrm{A}_{1}$ & A & $A_{1}$ & $\begin{array}{l}\frac{3}{3}\left|1+\lambda_{1}+\lambda_{2}\right|^{2} A_{1} \\
+\frac{1}{3}\left|2-\lambda_{1}-\lambda_{2}\right|^{2}= \\
+\frac{1}{2}\left|\lambda_{1}-\lambda_{2}\right|^{2} T_{2}\end{array}$ & $\begin{aligned} & \left.\frac{2}{3}\left|1+\lambda_{1}+\lambda_{2}\right|^{2}\right|_{1} \\
& +\frac{1}{3}\left|2-\lambda_{1}-\lambda_{2}\right|^{2} E \\
& +\left\{\frac{1}{2}\left|\lambda_{1}-\lambda_{2}\right|^{2}+2\left|\lambda_{3}\right|^{2}\right\} T_{2} \\
& +2\left|\lambda_{4}\right|_{1}^{2}\end{aligned}$ & $\begin{array}{l}\frac{2}{3}\left|1+\lambda_{1}+\lambda_{2}\right|^{2} A_{1} \\
+\frac{1}{3}\left|2-\lambda_{1}-\lambda_{2}\right|^{2}= \\
+\frac{1}{2}\left|\lambda_{1}-\lambda_{2}\right|^{2} T_{2}\end{array}$ \\
\hline $\mathrm{A}_{2}$ & B & $\mathrm{A}_{2}$ & $\left|\lambda_{3}\right|^{2} T_{1}+T_{2}$ & $\begin{array}{l}2 T_{2}+\left\{\left\{\left|\lambda_{5}\right|^{2}+\left|\lambda_{7}\right|^{2}\right\}_{1}\right. \\
+\left.2||_{1}\right|^{2} E\end{array}$ & $\left|\lambda_{3}\right|^{2} T_{1}+T_{2}$ \\
\hline$B_{1}$ & & $\mathrm{~B}_{1}$ & $\left|\lambda_{1}\right|_{2}^{2}+T_{2}$ & & $\left|\lambda_{4}\right|^{2} T_{1}+T_{2}$ \\
\hline $\mathrm{B}_{2}$ & & $\mathrm{~B}_{2}$ & $\left|\lambda_{5}\right| T_{1}+2 E$ & & $\mid \lambda_{5} T^{2} T_{1}+2 E$ \\
\hline
\end{tabular}


Table E-1 (Contd.)

\begin{tabular}{|c|c|c|c|c|c|}
\hline \multicolumn{2}{|c|}{$\mathrm{K}$} & M & & & \\
\hline $\mathrm{A}_{1}$ & A & $\mathrm{A}_{\mathrm{lg}}$ & $\begin{aligned} & \frac{2}{3}\left|1+\lambda_{1}+\lambda_{2}\right|^{2} A_{1} \\
& +\frac{1}{3}\left|2-\lambda_{1}-\lambda_{2}\right|^{2} E \\
+ & \frac{1}{2}\left|\lambda_{1}-\lambda_{2}\right|^{2} T_{2}\end{aligned}$ & $\begin{array}{l}\frac{2}{3}\left|I+\lambda_{1}+\lambda_{2}\right|^{2} \mathrm{~A}_{1} \\
+\frac{1}{3}\left|2-\lambda_{1}-\lambda_{2}\right|^{2} \mathrm{E} \\
\left.+\left\{\frac{1}{2}\left|\lambda_{1}-\lambda_{2}\right|^{2}+2 \mid \lambda_{3}\right)^{2}\right\} \mathrm{T}_{2} \\
+ \\
+2\left|\lambda_{1}\right|^{2} \mathrm{~T}_{1}\end{array}$ & $\begin{array}{l}\frac{2}{3}\left|1+\lambda_{1}+\lambda_{2}\right|^{2} \mathrm{~A}_{1} \\
+\frac{1}{3}\left|2-\lambda_{1}-\lambda_{2}\right|^{2} \mathrm{E} \\
+\frac{1}{2}\left|\lambda_{1}-\lambda_{2}\right|^{2} \mathrm{~T}_{2}\end{array}$ \\
\hline$A_{2}$ & B & $A_{2 g}$ & $\left|\lambda_{3}\right|^{2} \mathrm{~T}_{1}+\mathrm{T}_{2}$ & $\begin{array}{l}\left.2 \mathrm{~T}_{2}+\left\{2\left|\lambda_{0}\right|^{2}+\left|\lambda_{1}\right|\right\}^{2}\right\} T_{1} \\
+2\left|\lambda_{2}\right|^{2} \mathrm{E}\end{array}$ & $\mathrm{T}_{1}$ \\
\hline $\mathrm{B}_{1}$ & & ${ }^{B} 1 g$ & $\left|\lambda_{9}\right|^{2} T_{1}+T_{2}$ & & $6 \mathrm{E}+\mathrm{T}_{2}$ \\
\hline$B_{2}$ & & $\begin{array}{l}\mathrm{B}_{2 \mathrm{~g}} \\
\mathrm{E}_{\mathrm{g}}\end{array}$ & $\left|\lambda_{3}\right|^{2} T_{1}+2 E$ & & $\begin{array}{l}\mathrm{T}_{2} \\
3\left|\lambda_{2}\right|^{2} \mathrm{~T}_{1}+2 \mathrm{~T}_{2}+2 \mathrm{E}\end{array}$ \\
\hline
\end{tabular}



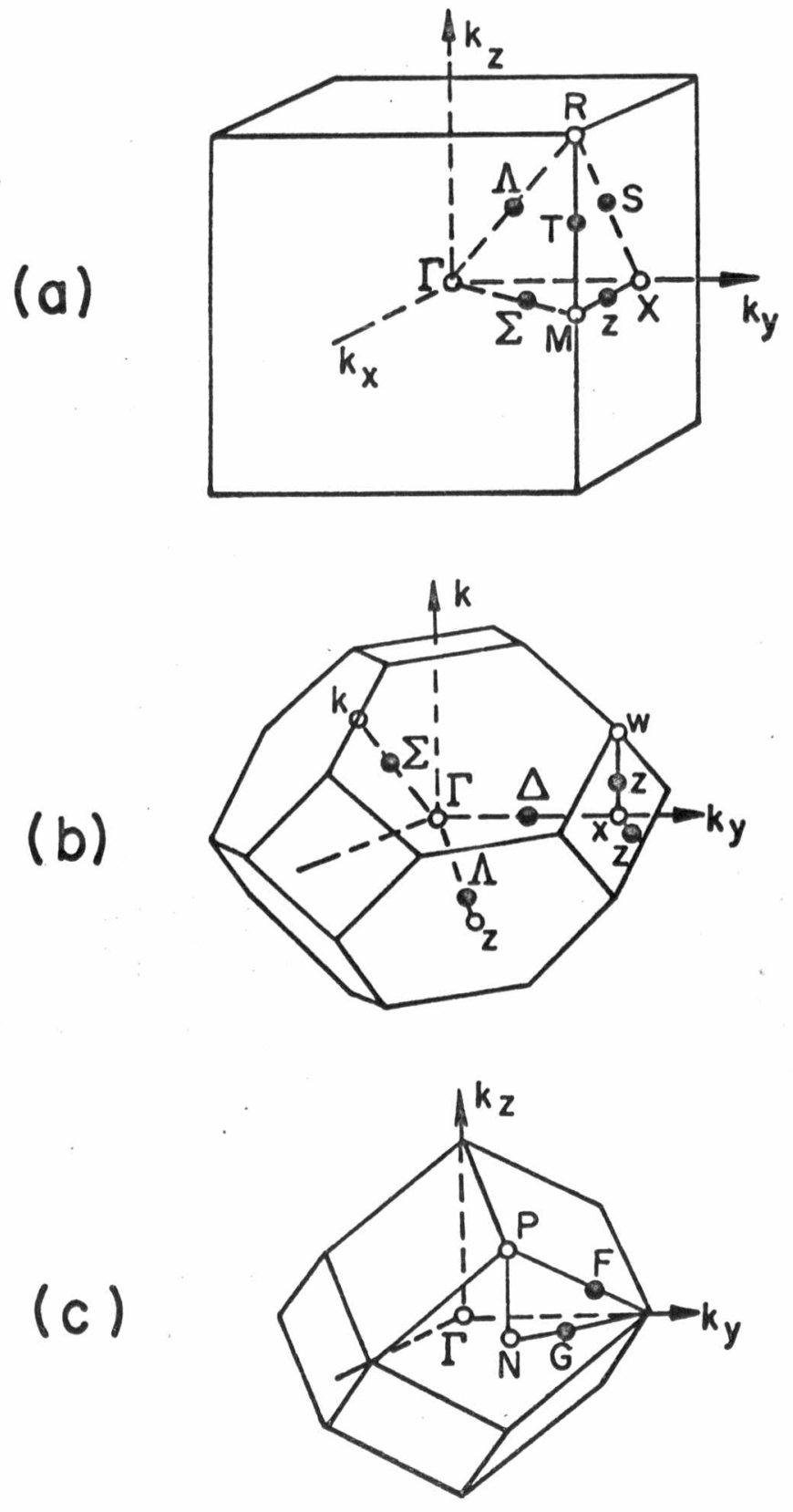

Fig.E-1. Brillouin zones of a) simple cubic structure, c) body-centered cubic structure and b) facecentered cubic, diamond and zinc blende structures 


\section{References}

1. J. C. Phillips, Solid State Physics, vol. 18, p. 55 (Academic Press, 1966).

2. P. A. Franken and J. F. Ward, Revs. of Mod. Phys. 35, 23 (1963).

3. M. Goeppert-Mayer, Ann. Physik 9, 273 (1931).

4. J. J. Hopfield and J. M. Worlock, Phys. Rev. 137, A1455 (1965).

5. H. R. Philipp and E. A. Taft, Phys. Rev. 113, 1002 (1959).

6. J. E. Eby, K. J. Teegarden and D. B. Dutton, Phys. Rev. 1161099 (1959).

7. D. I. Dexter, Photoconductivity Conference, R. G. Breckenridge et al., Eds. pp. I35-183 (Wiley, New York, 1956).

8. J. Tauc, Progress in Semiconductors, vol. 9, pp. 87-

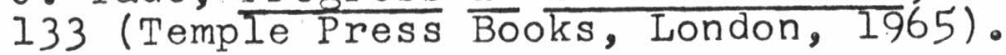

9. T. P. McLean, Progress in Semiconductors, vol. 5, p. $5 \dot{5}$ (John Wiley and Sons, Inc., New York, 1960).

10. G. Dresselhaus, Phys. Rev. 105, 135 (1956).

11. M. Inoue and Y Toyozawa, J. Phys. Soc. Japan 20, 363 (1965)。

12. D. Frohlich and B. Staginnus, Phys. Rev. Letters 19, 469 (1967)。

13. D. Frohlich and B. Staginnus and E. Schonherr, Phys. Rev. Letters 19, 1032 (1967).

14. M. Matsuoka, J. Phys. Soc. Japan 23, 1028 (1967).

15. D. Frohlich and B. Staginnus, to be published.

16. K. Park and R. G. Stafford, to be published. 
17. T. H. Reilly, thesis (Institute of Optics, University of Rochester), unpublished.

18. M. D. Galanin and Z. A. Chizhikova, Opt. Spektrosk, 113 (1967).

19. G. D. Mahan, Phys. Rev. Letters 20, 332 (1968).

20. G. D. Mahan, Phys. Rev. 170, 825 (1968).

21. L. I. Schiff, Quantum Mechanics, (McGraw-Hill Book Co., New York, 1949).

22. For a review of two-photon absorption see A. Gold, "Two Photon Spectroscopy", to be published in the Proceedings of the 1967 Varenna Summer School on Quantum Optics, R. T.Glauber, Ed.

23. P. A. M. Dirac, Proc. Roy. Soc. (London) Ser. A 114, $243(1927)$.

24. A thorough discussion of the quantized electromagnetic field is given in W. Heitler, The Quantum Theory of Radiation, (Oxford: Clarendon Press, 1954).

25. It should be noted here that coherence effects will be ignored. This is justified when one works with two beams of different frequency as long as the twophoton absorption is weak, which is generally the case. In the single beam case the absorption is smaller when the source is coherent than when it is chaotic. For a discussion of coherence effects see Y.R. Shen, Phys. Rev. 155, 921 (1967)。

26. R. J. Elliott, Phys. Rev. 108, 1384 (1957).

27. R. S. Knox, Theory of Excitons (Academic Press, New York, 1963).

28. J. Callaway, Energy Band Theory (Academic Press, New York, 1964).

29. J. C. Slater, Phys. Rev. 76, 1592 (1949); 87, 807 (1952).

30. G. F. Koster, Phys. Rev . 89, 67 (1953).

31. W. Kohn, Phys. Rev. 115, 809 (1959).

32. G. Wannier, Phys, Rev. 52, 191 (1937). 
33. G. Dresselhaus, J. Phys. Chem. Solids 1, 14 (1956).

34. G. Dresselhaus, Phys. Rev - 106, 76 (1956).

35. R. J. Elliott, T. P. Mclean and G. G. Macfarlane, Proc. Phys. Soc. 72, 553 (1958).

36. R. J. Elliott, Phys. Rev. 124, 340 (1961).

37. R. Braunstein, Phys. Rev. 125, 475 (1962).

38. R. Braunstein and N. Ockman, Phys. Rev. 134, A 499, (1964).

39. R. Loudon, Proc. Phys. Soc. 80, 952 (1962)。

40. L. P. Bouckaert, R. Smoluchowski and E. Wigner, Phys. Rev. 50, 100 (1936).

41. For a discussion of group theory and its application to solids see, for example, R. S. Knox and A. Gold, Symmetry in the Solid State, (W. A. Benjamin, Inc., New York, 1964$)$.

42. G. F. Koster, J. O.Dimmock, R. G. Wheeler and H. Statz, Properties of the Thirty-Two Point Groups (M.I.T.

43. G. F. Koster, in Solid State Physics (F. Seitz and D. Turnbull, Eds.) vol. 5, p. I73 (Academic Press, New York, 1957).

44. F. Seitz, Annals of Math 37, 17 (1936); Zeits. f. Kristallographie (A) 88, 433 (1934); 90, 289 (1935); 91, 336 (1935); 94, 100' (1936).

45. C. Herring, Journal of the Franklin Institute 233, $525(1942)$.

46. R. J. Elliott, Phys, Rev, 96, 280 (1954).

47. M. L. Glasser, J. Phys. Chem. Solids 10, 229 (1959).

48. R. C. Casella, Phys. Rev. 114, 1514 (1959)。

49. J. J. Hopfield, J. Phys. Chem. Solids 15, 97 (1960).

50. M. Balkanski and J. des Cloizeaux, J. Phys. Rad. 22, 41 (1961).

51. Y. Onodera, M. Okazaki and T. Inui, J. Phys. Soc. 
Japan 21, 2229 (1966).

52. L. Hostler, J. Math. Phys. 5, 591 (1964).

53. Handbook of Mathematical Functions, M. Abramowitz and I. A. Stegun, Eds. (U.S. Dept. of Commerce, N. B. S., Washington, D. C., 1964) Appl. Math. Ser. 55.

54. A. W. Overhauser, Phys. Rev. 101, 1702 (1956).

55. D. L. Dexter, Phys. Rev. 108, 707 (1957).

56. When a crystal has inversion symmetry there will be $2 n$ degenerate states at $k=0$, including spin, which will split into $n 2$-fold degenerate states. In crystals without inversion symmetry the $2 \mathrm{n}$ states will split into $2 n$ non-degenerate states when spin-orbit coupling is present. For a discussion of these splittings see V. Heine, Group Theory in Quantum Mechanics (Pergamon Press, 1960) Chapter VI, section 26.

57. W. Shockley, Phys. Rev • 50, 754 (1936).

58. M. K. Song, J. Physique 28, 195 (1967).

59. M. Cardona, Phys. Rev. 129, 69 (1963).

60. The group of the wave vector at $X$ is $D_{2 d}$. The representation labels used here are those of $R$. $H$. Parmenter, Phys. Rev. 100, 573 (1955), and are not numbered in order of those given in Table $3-1$. Parmenter's $X_{1}, X_{2}, X_{3}, X_{4}, X_{5}, X_{6}$, and $X_{7}$ correspond respectively to $A_{1}, B_{1}, B_{2}^{4}, A_{2}, E_{1}, \Gamma^{6}$ and $\Gamma^{7}$ of Tabie 3-1.

61. K. Park and H. S. Waff, Phys. Letters 28A, 305 (1968).

62. T. K. Bergstresser and M. L. Cohen, Phys. Rev. 164, 1067 (1967).

63. W. W. Piper, P. D. Johnson and D. T. F. Marple, J. Phys. Chem. Solids $\underline{8}, 457$ (1959).

64. D. G. Thornas and J. J. Hopfield, Phys. Rev. Il6, 573 (1959).

65. See, for example, R. S. Knox and K. Teegarden, "Electronic Excitations of Perfect Alkali Halide Crystals," in Physics of Color Centers (Academic Press, Inc., New York, I968). 
66. K. Teegarden and G. Baldini, Phys. Rev. 155, 896 (1967)。 67. Y. Onodera, J. Phys. Soc. Japan 25, 469 (1968).

68. J. J. Hopfield, J. M. Worlock and K. Park, Phys, Rev. Letters 11, 414 (1963). 